Moving to

Self-administered

Dietary Assessment

in

National Food

Consumption

Surveys

Liangzi Zhang

$>$ flip to unlock

$\because:$

$\wedge$ 


\section{Propositions}

1. Intentional behavioural change in dietary intake is the hardest challenge to solve in real-time dietary assessment. (this thesis)

2. The direct use of representative standard recipes is adequate in estimating population dietary intakes. (this thesis)

3. Evidence from peer-reviewed scientific publications comes too late for containing fastspreading novel infectious diseases.

4. The use of machine learning in cross-pollinating multidisciplinary data promotes more multidisciplinary research collaborations.

5. The better a public health system works, the less it is noticed.

6. Reasoning is an ineffective strategy to settle a family dispute.

Propositions belonging to the thesis, entitled

Moving to Self-administered Dietary Assessment in National Food Consumption Surveys Liangzi Zhang

Wageningen, 12 August 2020 



\section{Moving to Self-administered Dietary Assessment in National Food Consumption Surveys}

Liangzi Zhang 


\section{Thesis committee}

\section{Promotors}

Prof. Dr Hendriek Boshuizen

Special Professor of Biostatistic modelling

Wageningen University \& Research

\section{Co-promotor}

Dr Marga Ocké

Senior scientist, National institute for Public Health and the Environment (RIVM), Bilthoven

Senior Researcher, Division of Human Nutrition and Health, Wageningen University \& Research

\section{Other members}

Prof. Dr P. van 't Veer, Wageningen University \& Research

Dr A. Stafleu, Netherlands Nutrition Centre, The Hague

Prof. Dr J.E. Cade, University of Leeds, UK

Dr S.J.P.M. Eussen, Maastricht University

This research was conducted under the auspices of the Graduate School VLAG (Advanced studies in Food Technologies, Agrobiotechnology, Nutrition and Health Sciences) 


\title{
Moving to Self-administered Dietary Assessment in National Food Consumption Surveys
}

\author{
Liangzi Zhang
}

\section{Thesis}

submitted in fulfilment of the requirements for the degree of doctor at Wageningen University

by the authority of the Rector Magnificus,

Prof. Dr A.P.J. Mol,

in the presence of the

Thesis Committee appointed by the Academic Board

to be defended in public

on Monday 16 November 2020

at 11 a.m. in the Aula. 
Liangzi Zhang

Moving to Self-administered Dietary Assessment in National Food Consumption Surveys, 208 pages.

$\mathrm{PhD}$ thesis, Wageningen University, Wageningen, the Netherlands (2020)

With references, with summary in English

ISBN: 978-94-6395-527-0

DOI: https://doi.org/10.18174/530405 


\section{Contents}

$\begin{array}{ll}\text { Summary } & 7\end{array}$

Chapter $1 \quad$ General introduction 11

$\begin{array}{lll}\text { Chapter } 2 & \text { Importance of details in food descriptions in }\end{array}$

estimating population nutrient intake

distributions

Chapter 3

How does a simplified recipe collection

procedure in dietary assessment tools affect

the food group and nutrient intake

distributions of the population

Chapter 4

Evaluation of the recipe function in popular dietary

smartphone applications, with emphasize on features

relevant for nutrition assessment in large-scale

studies

Chapter 5

A systematic review and meta-analysis of validation studies performed on food record apps

Chapter 6 General discussion

181

Acknowledgements

199

About the author

203

List of publications 



\section{Summary}

The ever-growing findings from dietary studies have confirmed the important role of dietary intake in the development of certain non-communicable diseases (NCDs). In order to take a better control of the NCD progression in a population, primary prevention measures such as dietary guidelines and food policies are updated constantly according to the most recent scientific evidence and observed dietary patterns of a specific population. To obtain these dietary patterns, dietary intake at a national level is commonly monitored by governments in the form of food consumption surveys. However, assessing the dietary intake of a large population has been a challenging task throughout the years. With highly diversified food consumption practices and varied individual capabilities of reporting dietary intake, vast inputs (financially, physically) are required for collecting detailed dietary information. Hence, this thesis focuses on improving the methodology of dietary data collections, specifically for the Dutch National Food Consumption Surveys (DNFCS). The investigations proceeded in two parallel paths. Firstly, on how to remove burdensome procedures from current survey collection methods. Secondly, by learning from other studies and tools where dietary assessment techniques were investigated.

The current dietary assessment method in the DNFCS is the interviewer-administered 24hour recalls (24HRs) guided by the computer software called GloboDiet. Each food item goes through a round of detailed questions extracting relevant information (also called facets). This detail acquiring step has been the most time-consuming part of the interview. Besides, undesirable answers are likely to be obtained due to limited knowledge of the respondents. In order to enhance the interview efficiency while minimising the impact on the survey results, the importance of facets in terms of predicting the nutrient outcome was determined using a prediction model called random forest. As a result, $35 \%$ of the total facets were deemed unimportant and could be omitted; this would resulted in a change of $3.7 \%$ of the foods linked to the NEVO (Dutch Food Composition Database). The majority (79.4\%) of the differences between percentile estimates of the population nutrient intake distributions before and after facet deletion ranged from $0 \%$ to $1 \%$. The reduction of facets was estimated to save 637 hours for data collection and 442 hours for the data handling for a survey conducted on 3819 participants. However, facets that are informative for other food-related issues (e.g. food safety) should be carefully examined before deletion. (Chapter 2)

Another complicated task in the current GloboDiet 24HR interview is the recipe pathway. Typically, mixed dishes are firstly identified with a standard recipe, then the ingredient composition and amounts are adjusted according to the available information on the real dish eaten. A replacement of the burdensome recipe modifications with the unchanged standard recipes has been simulated in this study. Comparing the simulated results and the original dataset, the average of the absolute percentage difference for the population mean intakes was $1.6 \%$ across all food groups and $0.6 \%$ for nutrients. The soup group $(-6.6 \%)$ and 
docosahexaenoic acid (DHA) (-2.3\%) showed the largest percentage difference. The resulting small difference was mainly owning to the small proportion of energy intake consumed through mixed meals (10\%) among the Dutch population according to the survey. A list with more realistic standard recipes would enable the use of a simple recipe function in a self-administered 24hR or food diary. (Chapter 3)

With a fast-evolving smartphone industry and an increased awareness of diet-health relationships among the general public, large varieties of dietary recording apps have been developed and were made available for download from app stores. Since most of the apps were designed as self-administered instruments, their functionality might be a useful example for developing self-administered tools for large-scale nutritional monitoring or research. Out of 57 popular food record apps, 12 apps having a recipe function were scored according to pre-defined criteria. None of the apps provided adjustable standard recipes and applied retention factors to nutrients for heat-processed raw items. Energy and nutrient content from three random recipes were compared across apps and with NEVO. The variation in food composition databases (FCDs) underlying each app contributed the most to the differences larger than $5 \%$ of Daily Reference Intake (DRI) in $49 \%$ of the micronutrients and $20 \%$ of the energy and macronutrients. Applying retention factors decreased the nutrient content for specific heat-sensitive vitamins such as B6, B12, and folate up to $45 \%$. Overall, the components of current commercial apps vary, which might affect the accuracy of nutrient outcomes. In general, they focused more on the ease of use than getting accurate information. (Chapter 4)

Different from commercial apps, that have been mostly compared with each other, researchbased apps have been described more in detail, reporting on their development, validity compared with a reference method, and usability or feasibility of applications in a sample population. A systematic review and meta-analysis on validation studies therefore provides insights into the general applicability and potentially the common flaws of apps. From an online search of literatures from 2013 to 2019, 14 studies were found that have validated food record apps in real-life settings. The pooled mean difference between the apps and the reference methods across studies showed a general underestimation of energy intake (-202 kcal/day) by using the apps. Studies with different FCDs for each method had the largest mean differences. The sources of variation were traced for studies that compared food group/ food item differences. A variation in study designs has been found among studies, which impedes the comparisons across studies, (e.g. use of energy-adjusted/log-transformed values). In general, most studies did not comply with the recommended procedures for conducting validation studies. (Chapter 5)

In Chapter 6, we discuss the implications and methodological reflection combining the findings from previous chapters. Other aspects related to dietary assessment, such as technology evolvement, data privacy, future directions are also discussed. Lastly, based on 
the evidence from our studies and other literatures, we come up with a recommended procedure for developing new self-administered methods for NFCS in general.

In conclusion, this thesis has shown that a simplification of current interviewer-administered $24 \mathrm{HR}$ is promising, which implies that the simplified functions might work equally well in a more cost-effective self-administered method. The advanced features and prevalence of use have made smartphones the optimal platform for monitoring dietary intakes at a population level. Still, a larger underestimation of energy intake using self-administered methods is expected compared to interviewer-administered methods, which implies the need for more guidance compared to using commercial apps, and careful interpretation of results. The validity of apps should be tested among different age groups, and a compatible option for those having difficulties in completing the survey by themselves should be considered. Moving to a self-administered method is a big step for NFCS, which requires careful considerations and large inputs during the development and validation phase. However, the lower costs and efforts required by using self-administered method could highly likely to counterbalance the initial investment, in the meanwhile, providing participants with a more flexible platform for dietary recording. 

Chapter 1

General introduction 


\section{National Food Consumption Surveys: General Use and Challenges}

In recent decades, the increasing prevalence of non-communicable diseases worldwide (e.g. cardiovascular disease, stroke, type 2 diabetes, and some cancers) induces enormous economic and social burden. These diseases are currently contributing to around $75 \%$ of all death worldwide (1). Overweight and obesity are one of the major cardiometabolic risk factors closely associated with unhealthy diet (2). Therefore, there has been a shift in focus of health authorities from disease treatments to primary prevention by assessing and managing dietary patterns $(3,4)$. The dietary patterns of a specific population can be derived from food consumption surveys that capture the detailed consumption of foods, beverages, and supplements at individual levels (5). National-scale food consumption surveys became to be the main source of information on the prevalence of dietary risk factors at a population level and have been increasingly conducted across countries worldwide (6). They are important basis for policy-making, providing insights into the dietary practices of the population, and enable evaluation of compliance with dietary guidelines, and inform on the appropriateness of food policies (7-9). Equally important, nutrition surveys can provide information on the exposure to food-related hazards and emerging risks to inform updates on food safety legislation (10).

Nutrition surveys typically consist of, firstly, collecting food consumption from a representative sample of the population using a dietary assessment instrument, secondly, obtaining nutrient information by linking reported food items to food composition databases (FCDs) (11). The data collection step is especially challenging, due to the vast varieties of available foods and unbalanced participation rates from different population groups. Besides, errors made intentionally as well as unintentionally by subjects can easily occur when perceiving and reporting the kind and the amount of food they consumed (12). Such measurement error can be divided into random or systematic. Random-errors (e.g. day-to-day variation of intakes) reduce the precision of the measurement, resulting in a loss in statistical power. Loss of power, however, could be mitigated with large-enough sample sizes and repeated measurements. Systematic errors generate bias (e.g. underreporting), can be intakerelated or person-specific, and can only be identified and corrected with a reference method that is preferably free of error (13-15). Hence, a successful collection of large-scale data should take both types of measurement error into account, require substantial investment in time and cost, and has been a challenge for government institutions, researchers, and dietitians (16).

\section{National Food Consumption Surveys in Europe}

National food consumption surveys are presently carried out in many European countries and provide valuable information on dietary patterns and food safety at both national and EU level. The most frequently used dietary assessment methods in Europe for collecting national food 
consumption data are $24 \mathrm{HRs}$ and food records (2). Both open methods can provide detailed information on the intake of all foods and drinks on a specific day(s). 24HRs depend on the subjects' ability to recall all foods and portion sizes consumed over a reference period of one day, and were traditionally conducted in person or by telephone interviews with a trained interviewer following a structured protocol that facilitate participants in recalling $(17,18)$. On the other hand, food records require participants to self-report food consumption in realtime. Although this prevents errors associated with memory loss, food records suffer from behaviour change and misreporting, due to reactivity bias and social desirability bias, respectively (19). To facilitate complete and detailed recording, careful in-person training of participants before data collection and data reviewing by researchers afterwards poses additional burdens for this method (20). Both short-term methods have limited ability in capturing episodically consumed foods (21). Hence, multiple days of measurement in combination with a food frequency questionnaire (FFQ) were suggested as inputs for statistical techniques developed for usual dietary intake estimations (11, 22-25). The European Food Safety Authority advises EU Member states to collect two non-consecutive $24 \mathrm{hRs}$ for adults and two non-consecutive food records for children (26).

The demand for a structured and standardised collection of dietary intake data in national nutrition surveys has led to a wide application of Computer Assisted Interview (CAI) software. A computer-assisted 24HR interview software Automated Multiple-Pass Method (AMPM), developed and validated by the U.S. Department of Agriculture (USDA), was used in the National Health and Nutrition Examination Survey in the US (7). Whereas a validated (27) and standardized software GloboDiet (formerly known as Epic-Soft), was used by some European countries for the aim of collecting harmonized data among the EU Member states (28-30).

\section{GloboDiet Features}

The current Dutch National Food Consumption Survey (DNFCS) follows the standard protocol of GloboDiet, with adjusted food lists, probe questions and facet-descriptor system specific to the Dutch dietary culture and available food products (31). The flow diagram of the 24HR procedure is shown in Figure 1. Firstly, a quick list of all consumed foods throughout the recalled day is generated. Then, the facet-descriptor system (e.g. preservation method, fortification, etc.) enables the interviewers to collect detailed information for each food item. Sufficiently detailed dietary intake data enable more accurate nutrient estimations. They are also required for adequate exposure assessment of food contaminants, fortification and environmental impact because exposure levels vary widely due to variation in food processing, preservation, cooking, etc. (32). Meanwhile, probe questions recover food items and eating occasions not reported initially, such as common additions to foods (e.g., butter on toast) and snacks. The effectiveness of these probes is well-established and is therefore part of the interviewing protocols for all standardised high-quality 24HRs (33). An early study 
found that respondents with interviewer probing reported $25 \%$ higher dietary intakes than did respondents without interviewer probing (34).

Foods typically eaten as mixed dishes consist of multiple ingredients with specific food preparation and often with cooking involved (35). For respondents, it might be difficult to accurately describe the types and amounts of the various ingredients in mixed dishes, especially for those who were not involved in cooking (36). Standard recipe databases are often used in national food consumption surveys to ease the recording of mixed dishes (37). The possibility to modify the standard recipes, if a participant can report the specific recipe, is part of a comprehensive recipe function in GloboDiet which involves ingredient identification and quantity calculation with the presence of an interviewer (Figure 1). The accurate calculation of nutrients for a cooked food takes weight change and nutrient loss due to cooking and processing into account (38). The GloboDiet program calculates the cooked amount of ingredients from raw amount using pre-defined algorithms and standard foodspecific coefficients (e.g., raw-to-cooked yield factors, or edible part coefficients)(29, 30). The cooked amounts are then multiplied by the nutrient values of the cooked food items found in the Dutch food composition table (NEVO)(38).

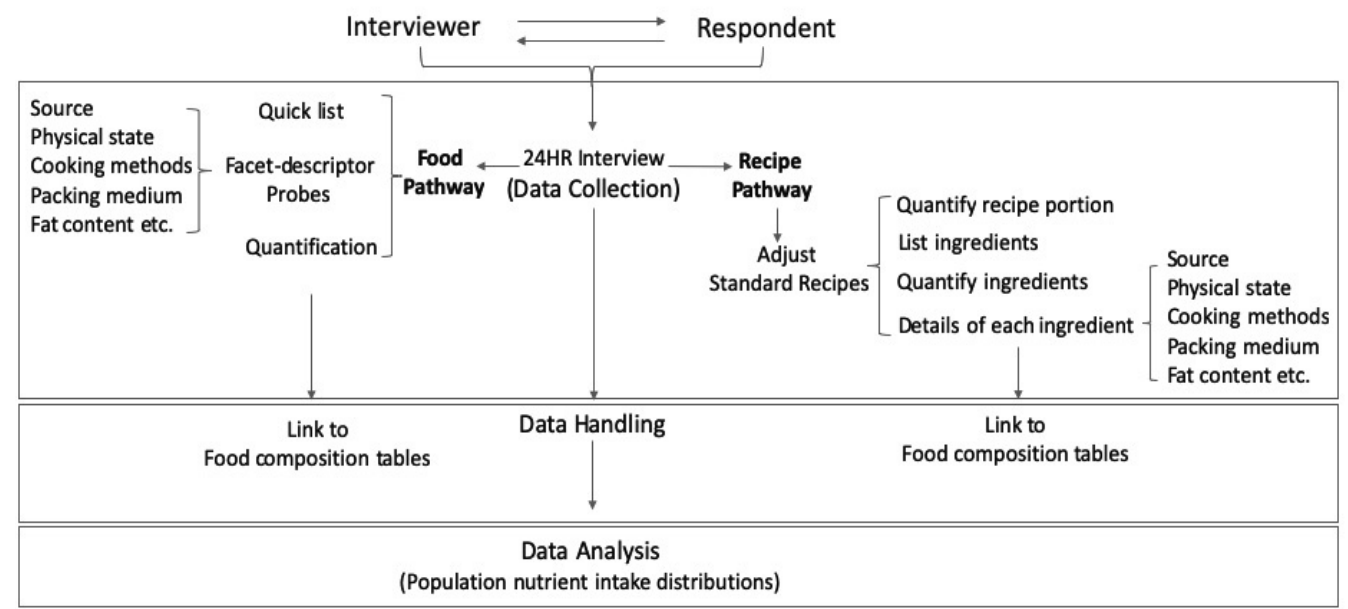

Figure 1. Main steps of the 24HR interview procedure using GloboDiet (based on Slimani et al. (39)), including data collection, handling and analysis.

\section{Limitations of Interviewer-administered 24HR}

An interviewer-administered 24-hour recall interview using the multiple-pass approach typically requires between 30 and 45 minutes (40). Interviewers must be highly trained and experienced to collect high-quality data that provide information that fits the study purposes. In addition, the procedures in the current method have limitations in time and location for data collection, all these features together induce high costs $(12,41)$. Besides, the requirement of knowing many details of the food/recipe consumed will lengthen the interview, which is 
at odds with increasing the participation rate of the survey, and might also cause intentional under-reporting. Moreover, apart from involuntary food omission, the lack of ability to conceptualise portion sizes based on memory is another main source of recall bias that could not be solved easily in current data collections (42). These limitations, together with the high cost and efforts for the survey implementation, have contributed to the initiatives to simplify the collection and handling of dietary data in national food consumption surveys $(43,44)$.

\section{New Opportunities with Technologies}

Continued development in ICTs and increased ubiquity of computer and portable devices led to investigations into self-administered tools to overcome the high cost and reliance on highly trained interviewers, reduce respondents' burden and increase the efficiency of data collection and handling (45). Internet-based dietary assessment software has been increasingly developed that require respondent themselves (rather than a trained nutritionist) to correctly identify and select the appropriate food or drink item that they have consumed (46). These self-administered tools usually provide tutorials, digital images for food identification and portion-size estimation, and various audio files, to facilitate the data collection procedures (12). In terms of linkage to FCDs, compared to manual linkage in some interviewer-assisted methods (e.g. GloboDiet), efforts were also reduced in self-administered methods due to the already-established linkage between food items and options in FCDs before the data collection (47).

More recently, the advent of mobile devices allowed more functionalities beyond text-based systems to be incorporated, such as barcode scanning and image capturing, which requires less time and effort on the part of respondents, and are less subjective compared to descriptions provided by respondents $(16,48,49)$. Among the available mobile devices (e.g. PDA, tablets, etc.), smartphones are the most prevalent tool that reached a global penetration of $41.5 \%$ in 2020, while the Netherlands is forecasted to reach 96 percent as of 2024 (50). The accessibility and popularity of diet and healthy lifestyle applications (known as "apps") opened a new array of possibilities for innovative applications for dietary recording (51). A wide variety of food record apps became available to increase the awareness of the type of food consumed and facilitate body weight control or disease management with personalised advice provided (52). In the research domain, apps can enable the measuring of food and nutrient intakes in real-time from large populations at a relatively low cost, with automated calculation of daily food and nutrient intake and less interviewer involvement. Participants have greater flexibility and fewer time constraints to complete the survey because users usually carry smartphones with them $(44,46)$. The advantages of using smartphones for dietary assessment has prompted researchers investigating the opportunities for their applications in epidemiological research and nutrition monitoring (53-57). 
Apart from an exclusive self-reported electronic food diary, photo-assisted food records with or without analysis by dieticians and automatic analysis of digital food images have also been actively investigated (56). The images provide objective information such as food type, volume, and leftovers, and may even record foods that were forgotten and not reported in the food registration, hence can be used as a supplement to traditional written or electronic food records $(58,59)$. Although image-assisted methods minimise participant and researcher burden to some extent during data collection, the amount of data influx is vast and requires additional work from researchers for data cleaning (60). On the other hand, advanced computer vision has enabled the development of automatic image recognition (61). However, computer vision methods still exhibit practical limitations, such as a shortage of food images that are representative of a specific diet for training the algorithm. Hence, a higher level of maturity is required before they can be used as the main dietary assessment method (62).

Alternative methods for detecting eating behaviour or food consumption are based on sensors. One type of sensor could detect noises of chewing or swallowing when placed on the ear or the neck (63). More recent miniaturised tooth-mounted radiofrequency sensors are capable of detecting nutrients and wirelessly communicating to a mobile device (64). There are also devices attached to the arm for detecting movement for eating behaviours using magnetic proximity and infrared sensors (65). However, these sensors are often intrusive, uncomfortable and/or cosmetically unpleasant for long-term wear. Less intrusive methods including using smart kitchen equipment (e.g., plates, spoons, and tables) to identify food items and weight before and after meal consumption (66). Another miniaturised hand-held (near-infrared) spectrometers could determine the characteristic of food matrix properties by scanning food items (67). However, the applications of these sensor-based devices were limited to controlled-settings and are still immature to be applied in larger samples of freeliving individuals (67). The cost of these devices has not been established since they are still in the development phase and have not gone further to establish a market cost (9). Besides, their inability to recognize all foods and nutrients is the main impediment for current sensors being the main dietary assessment method. Some examples of available technologies in sensor-based technologies are listed in Figure 2. 


\section{Smartphone-based}

technologies

\section{Some examples of}

Sensor-based

technologies

- Voice sensor (chew, swallow...)

- Movement sensor (eating behaviors)

- Kitchenware sensor (plate, spoon...)

- Wearable camera

- Radiofrequency sensor

- Near-infrared spectrometers

Figure 2. Examples of technologies used in the smartphone- and sensor-based dietary assessment methods.

\section{Development and Evaluation of Smartphone Apps}

With the increasing amount of smartphone dietary apps, there is a corresponding rise in the number of studies that evaluate apps in terms of their accuracy, usability and behavioural change impact (68). Owing to the large varieties of app design, more multidimensional quality assessments have been found in addition to conventional evaluations, such as on functionality, popularity, adherence to self-monitoring etc. In terms of content accuracy, the evaluations focusing on assessing the quality of the underlying food composition database and associated nutrient calculation algorithms have been published for most commercial apps $(68,69)$. However on some specific functionalities, like recipe functions, evaluations are missing. On the other hand, for apps developed for research purposes, the development process, feasibility, usability and validity are more commonly assessed $(45,54,70)$. The level of validity refers to the degree to which the new method measures what it intends to measure quantitively (71). Investigating the validity of new methods is crucial, given the complexity of our diet and multiple sources of bias that impact the nutrient outcomes of dietary assessments. The result of using a test method, in this case the app, should be compared with a reference method that has a greater degree of demonstrated validity and has uncorrelated errors with the test method (17). A summary on the study design and nutrient comparisons of validation studies could provide useful information on the likelihood of applying a certain type of apps to a specific study purpose (e.g. in NFCS).

\section{Aim and outline of this thesis}

As discussed before, traditional dietary assessment methods are subjected to both random and systematic errors, mainly owing to self-reporting. Meanwhile, dietary assessment methods that are open in nature induce a heavy burden on both the researchers and 
respondents, especially for a detailed dietary data collection at a large scale. Developing a more cost-effective method taking advantages of current technologies is the foremost task for NFCS conducted in most countries, resulting in a trend of moving from an intervieweradministered to a self-administered dietary assessment method. Among currently available devices, smartphones show a great potential to be applied in DNFCS with their growing functionalities and data processing capacities. Therefore, to enhance the cost-efficiency of DNFCS in data collection and handling, a more efficient and flexible method built in smartphones has been proposed. This thesis includes investigations into the proposed component configurations and review of other evidence to deliver support for future app development.

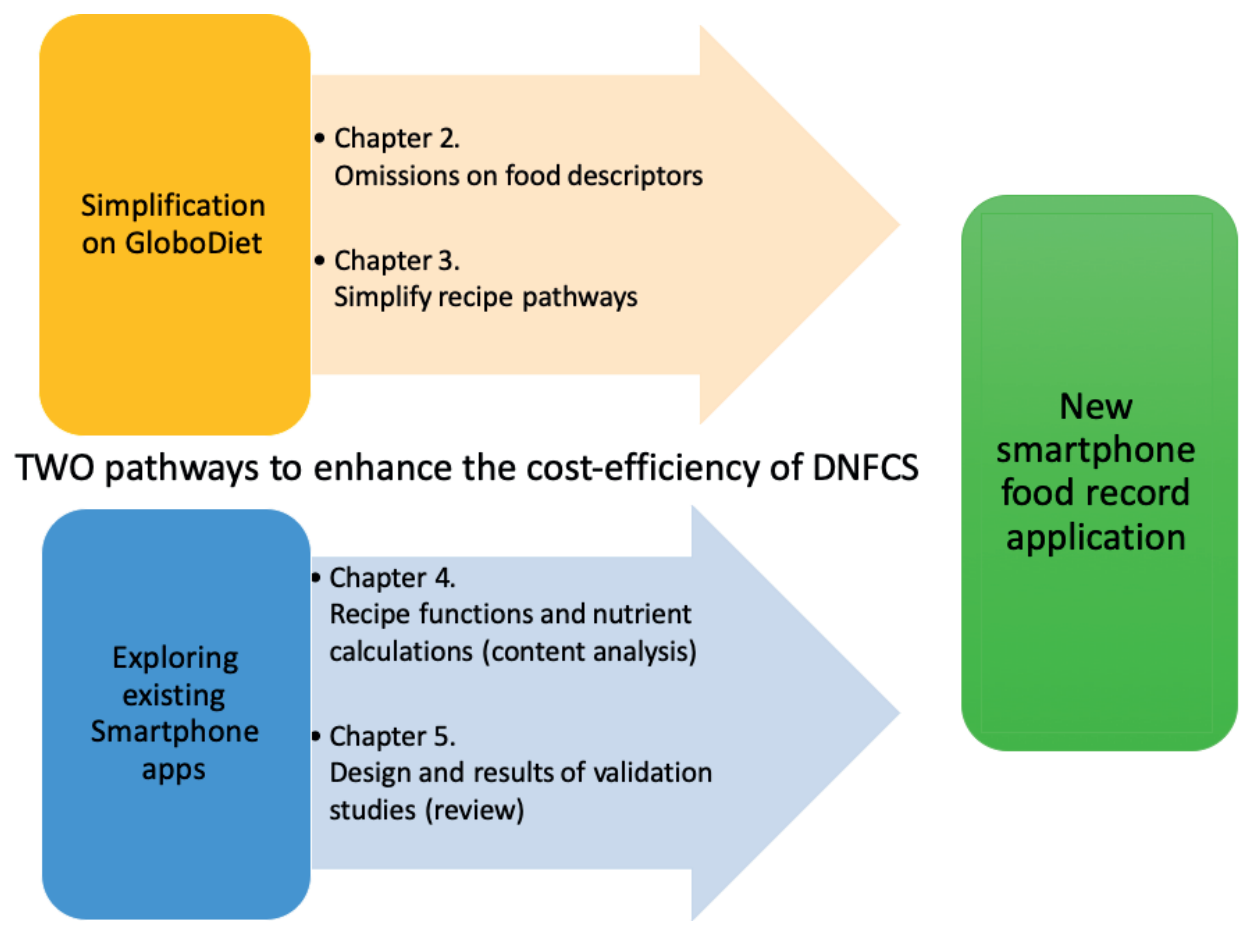

Figure 3. Two parallel paths for methodology development of switching from interviewer-assisted 24HR to self-administered smartphone food records.

Figure 3 illustrates two parallel paths assisting methodological transformations from interviewer-assisted 24HRs to self-administered smartphone food records in DNFCS. In the first path, the level of accuracy and efficiency resulting from the component simplification of existing methods were evaluated. Chapter 2 identified less important food descriptions (facets) in GloboDiet, and evaluated the influence of the facet reduction on nutrient intake distributions of the population and the extent of time-saving in a simulation study. In chapter 3 , a simulation on removing complicated steps of recording consumed mixed meal 
composition from GloboDiet is presented, looking at the impact this has on the food group and nutrient intake distributions of the Dutch population.

In the second path, evidence on the available technologies from other studies are summarised and combined with the existing innovation propositions in DNFCS. Chapter 4 summarises the mixed meal recording features in several popular food diary apps from a research perspective and their accuracy in estimating nutrient intakes compared to the Dutch Food Composition Database (NEVO). Chapter 5 systematically reviews the existing validation studies of food record apps concerning their study designs, and pools the results of nutrient comparisons between the apps and the respective reference methods. In the final chapter of this thesis, Chapter 6, the main findings of the chapters are summarised and discussed. This chapter also includes recommendations for future research. 


\section{Reference}

1. Arens-Volland AG, Spassova L, Bohn T. Promising approaches of computersupported dietary assessment and management-Current research status and available applications. Int J Med Inform. 2015;84(12):997-1008.

2. Rippin H, Hutchinson J, Evans C, Jewell J, Breda J, Cade J. National nutrition surveys in Europe: a review on the current status in the 53 countries of the WHO European region. Food \& Nutrition Research. 2018;62.

3. Mozaffarian D, Wilson PW, Kannel WB. Beyond established and novel risk factors: lifestyle risk factors for cardiovascular disease. Circulation. 2008;117(23):3031-8.

4. Organization WH. European food and nutrition action plan 2015-2020. 2015.

5. Kroes R, Muller D, Lambe J, Lowik MR, van Klaveren J, Kleiner J, et al. Assessment of intake from the diet. Food Chem Toxicol. 2002;40(2-3):327-85.

6. Lim SS, Vos T, Flaxman AD, Danaei G, Shibuya K, Adair-Rohani H, et al. A comparative risk assessment of burden of disease and injury attributable to 67 risk factors and risk factor clusters in 21 regions, 1990-2010: a systematic analysis for the Global Burden of Disease Study 2010. Lancet. 2012;380(9859):2224-60.

7. Ahluwalia N, Dwyer J, Terry A, Moshfegh A, Johnson C. Update on NHANES Dietary Data: Focus on Collection, Release, Analytical Considerations, and Uses to Inform Public Policy. Advances in Nutrition. 2016;7(1):121-34.

8. Rollo ME, Williams RL, Burrows T, Kirkpatrick SI, Bucher T, Collins CE. What Are They Really Eating? A Review on New Approaches to Dietary Intake Assessment and Validation. Current Nutrition Reports. 2016;5(4):307-14.

9. Archundia Herrera MC, Chan CB. Narrative Review of New Methods for Assessing Food and Energy Intake. Nutrients. 2018;10(8).

10. Ambrus Á, Horváth Z, Farkas Z, Dorogházi E, Cseh J, Petrova S, et al. Pilot study in the view of a Pan-European dietary survey - adolescents, adults and elderly. EFSA Supporting Publications. 2013;10(11):508E-n/a.

11. Gibson RS. Principles of nutritional assessment: Oxford university press, USA; 2005.

12. Shim J-S, Oh K, Kim HC. Dietary Assessment Methods in Epidemiologic Studies. Epidemiology and Health. 2014;36:e2014009.

13. Thompson FE, Kirkpatrick SI, Subar AF, Reedy J, Schap TE, Wilson MM, et al. The National Cancer Institute's Dietary Assessment Primer: A Resource for Diet Research. J Acad Nutr Diet. 2015;115(12):1986-95.

14. Subar AF, Crafts J, Zimmerman TP, Wilson M, Mittl B, Islam NG, et al. Assessment of the accuracy of portion size reports using computer-based food photographs aids in the development of an automated self-administered 24-hour recall. J Am Diet Assoc. 2010;110(1):55-64.

15. Freedman LS, Schatzkin A, Midthune D, Kipnis V. Dealing with dietary measurement error in nutritional cohort studies. J Natl Cancer Inst. 2011;103(14):1086-92. 
16. Tay W, Kaur B, Quek R, Lim J, Henry CJ. Current Developments in Digital Quantitative Volume Estimation for the Optimisation of Dietary Assessment. Nutrients. 2020;12(4):1167.

17. Willett W, Lenart E. Reproducibility and validity of food-frequency questionnaires. Nutritional epidemiology. 2013:96-141.

18. Zimmerman TP, Hull SG, McNutt S, Mittl B, Islam N, Guenther PM, et al. Challenges in converting an interviewer-administered food probe database to selfadministration in the National Cancer Institute Automated Self-administered 24-Hour Recall (ASA24). Journal of food composition and analysis : an official publication of the United Nations University, International Network of Food Data Systems. 2009;22(Suppl 1):S48-S51.

19. Macdiarmid J, Blundell J. Assessing dietary intake: Who, what and why of underreporting. Nutrition research reviews. 1998;11(2):231-53.

20. Kolar AS, Patterson RE, White E, Neuhouser ML, Frank LL, Standley J, et al. A practical method for collecting 3-day food records in a large cohort. Epidemiology. 2005;16(4):579-83.

21. Illner AK, Nothlings U, Wagner K, Ward H, Boeing H. The assessment of individual usual food intake in large-scale prospective studies. Ann Nutr Metab. 2010;56(2):99105 .

22. Conrad J, Nothlings U. Innovative approaches to estimate individual usual dietary intake in large-scale epidemiological studies. P Nutr Soc. 2017;76(3):213-9.

23. Satoh M, Tanno Y, Hosaka M, Metoki H, Obara T, Asayama K, et al. Salt intake and the validity of a salt intake assessment system based on a 24-h dietary recall method in pregnant Japanese women. Clinical and Experimental Hypertension. 2015;37(6):459-62.

24. Dodd KW, Guenther PM, Freedman LS, Subar AF, Kipnis V, Midthune D, et al. Statistical methods for estimating usual intake of nutrients and foods: a review of the theory. J Am Diet Assoc. 2006;106(10):1640-50.

25. Carriquiry AL. Understanding and Assessing Nutrition. Annu Rev Stat Appl. 2017;4:123-46.

26. Guidance on the EU Menu methodology1 European Food Safety Authority.

27. Crispim SP, Geelen A, Souverein OW, Hulshof PJ, Ruprich J, Dofkova M, et al. Biomarker-based evaluation of two 24-h recalls for comparing usual fish, fruit and vegetable intakes across European centers in the EFCOVAL Study. Eur J Clin Nutr. 2011;65 Suppl 1:S38-47.

28. Crispim SP, de Vries JH, Geelen A, Souverein OW, Hulshof PJ, Lafay L, et al. Two non-consecutive $24 \mathrm{~h}$ recalls using EPIC-Soft software are sufficiently valid for comparing protein and potassium intake between five European centres--results from the European Food Consumption Validation (EFCOVAL) study. Br J Nutr. 2011;105(3):447-58. 
29. Slimani N, Deharveng G, Charrondiere RU, van Kappel AL, Ocke MC, Welch A, et al. Structure of the standardized computerized 24-h diet recall interview used as reference method in the 22 centers participating in the EPIC project. European Prospective Investigation into Cancer and Nutrition. Comput Methods Programs Biomed. 1999;58(3):251-66.

30. EFSA. Guidance on the EU Menu methodology. EFSA Journal2014.

31. Slimani N, Casagrande C, Nicolas G, Freisling H, Huybrechts I, Ocke MC, et al. The standardized computerized 24-h dietary recall method EPIC-Soft adapted for panEuropean dietary monitoring. Eur J Clin Nutr. 2011;65 Suppl 1:S5-15.

32. EFSA. General principles for the collection of national food consumption data in the view of a pan-European dietary survey. EFSA Journal. 2009;7(12):1435.

33. Thompson FE, Subar AF, Boushey CJ, Ferruzzi MG, Delahanty LM. Chapter 1 Dietary Assessment Methodology A2 - Coulston, Ann M. Nutrition in the Prevention and Treatment of Disease (Fourth Edition): Academic Press; 2017. p. 5-48.

34. Campbell VA, Dodds ML. Collecting dietary information from groups of older people. J Am Diet Assoc. 1967;51(1):29-33.

35. Kong K, Zhang L, Huang L, Tao Y. Validity and practicability of smartphone-based photographic food records for estimating energy and nutrient intake. Asia Pac J Clin Nutr. 2017;26(3):396-401.

36. Schober DJ, Carpenter L, Currie V, Yaroch AL. Evaluation of the LiveWell@School Food Initiative Shows Increases in Scratch Cooking and Improvement in Nutritional Content. J School Health. 2016;86(8):604-11.

37. Imaeda N, Goto C, Fujiwara N, Kato R, Tokudome Y. Comparison of Nutrient Contents between the Standard Recipes Used in the National Health and Nutrition Survey and the Dishes Actually Consumed by Community-dwelling Elderly Individuals. Journal of Japanese Society of Nutrition \& Food Science. 2016;69(5):237-48.

38. Reinivuo H, Bell S, Ovaskainen ML. Harmonisation of recipe calculation procedures in European food composition databases. J Food Compos Anal. 2009;22(5):410-3.

39. Slimani N, Ferrari P, Ocke M, Welch A, Boeing H, Liere M, et al. Standardization of the 24-hour diet recall calibration method used in the european prospective investigation into cancer and nutrition (EPIC): general concepts and preliminary results. Eur J Clin Nutr. 2000;54(12):900-17.

40. Thompson FE, Subar AF, Loria CM, Reedy JL, Baranowski T. Need for technological innovation in dietary assessment. J Am Diet Assoc. 2010;110(1):48-51.

41. Thompson FE, Subar AF. Dietary assessment methodology. Nutrition in the Prevention and Treatment of Disease: Elsevier; 2017. p. 5-48.

42. Forman M, Zhang J, Gunter E, Yao S, Gross M, Qiao Y, et al. Season-specific Correlation between Dietary Intake of Fruits and Vegetables and Levels of Serum Biomarkers among Chinese Tin Miners at High Risk for Lung Cancer. Annals of the New York Academy of Sciences. 1999;889(1):230-9. 
43. Ocke MC, Slimani N, Brants H, Buurma-Rethans E, Casagrande C, Nicolas G, et al. Potential and requirements for a standardized pan-European food consumption survey using the EPIC-Soft software. European Journal of Clinical Nutrition. 2011;65:S48S57.

44. Touvier M, Kesse-Guyot E, Méjean C, Pollet C, Malon A, Castetbon K, et al. Comparison between an interactive web-based self-administered $24 \mathrm{~h}$ dietary record and an interview by a dietitian for large-scale epidemiological studies. British Journal of Nutrition. 2010;105(7):1055-64.

45. Subar AF, Thompson FE, Potischman N, Forsyth BH, Buday R, Richards D, et al. Formative research of a quick list for an automated self-administered 24-hour dietary recall. J Am Diet Assoc. 2007;107(6):1002-7.

46. Carter MC, Burley VJ, Nykjaer C, Cade JE. 'My Meal Mate' (MMM): validation of the diet measures captured on a smartphone application to facilitate weight loss. Br J Nutr. 2013;109(3):539-46.

47. Naska A, Lagiou A, Lagiou P. Dietary assessment methods in epidemiological research: current state of the art and future prospects. F1000Research. 2017;6:926.

48. Maringer M, Wisse-Voorwinden N, Veer PV, Geelen A. Food identification by barcode scanning in the Netherlands: a quality assessment of labelled food product databases underlying popular nutrition applications. Public Health Nutr. 2018:1-8.

49. Cade JE. Measuring Diet in the 21 st Century: Use of New Technologies. P Nutr Soc. 2017;76(3):276-82.

50. Statista. Forecast of smartphone user numbers in the Netherlands 2018-2024 (in millions) 2020 [Available from: https:/www.statista.com/statistics/494636/smartphone-users-in-netherlands/.

51. Long JD, Littlefield LA, Estep G, Martin H, Rogers TJ, Boswell C, et al. Evidence review of technology and dietary assessment. Worldviews on Evidence-Based Nursing. 2010;7(4):191-204.

52. Foster E, Lee C, Imamura F, Hollidge SE, Westgate KL, Venables MC, et al. Validity and reliability of an online self-report 24-h dietary recall method (Intake24): a doubly labelled water study and repeated-measures analysis. J Nutr Sci. 2019;8:e29.

53. Ambrosini GL, Hurworth M, Giglia R, Trapp G, Strauss P. Feasibility of a commercial smartphone application for dietary assessment in epidemiological research and comparison with 24-h dietary recalls. Nutr J. 2018;17(1):5.

54. Eldridge AL, Piernas C, Illner AK, Gibney MJ, Gurinovic MA, de Vries JHM, et al. Evaluation of New Technology-Based Tools for Dietary Intake Assessment-An ILSI Europe Dietary Intake and Exposure Task Force Evaluation. Nutrients. 2019;11(1).

55. Fallaize R, Zenun Franco R, Pasang J, Hwang F, Lovegrove JA. Popular NutritionRelated Mobile Apps: An Agreement Assessment Against a UK Reference Method. JMIR Mhealth Uhealth. 2019;7(2):e9838.

56. Sharp DB, Allman-Farinelli M. Feasibility and validity of mobile phones to assess dietary intake. Nutrition. 2014;30(11-12):1257-66. 
57. Allman-Farinelli M, Gemming L. Technology Interventions to Manage Food Intake: Where Are We Now? Current Diabetes Reports. 2017;17(11):103.

58. O'Loughlin G, Cullen SJ, McGoldrick A, O'Connor S, Blain R, O'Malley S, et al. Using a Wearable Camera to Increase the Accuracy of Dietary Analysis. American journal of preventive medicine. 2013;44(3):297-301.

59. Gemming L, Utter J, Mhurchu CN. Image-Assisted Dietary Assessment: A Systematic Review of the Evidence. Journal of the Academy of Nutrition and Dietetics. 2015;115(1):64-77.

60. McClung HL, Champagne CM, Allen HR, McGraw SM, Young AJ, Montain SJ, et al. Digital food photography technology improves efficiency and feasibility of dietary intake assessments in large populations eating ad libitum in collective dining facilities. Appetite. 2017;116:389-94.

61. Ming Z-Y, Chen J, Cao Y, Forde C, Ngo C-W, Chua TS, editors. Food Photo Recognition for Dietary Tracking: System and Experiment. International Conference on Multimedia Modeling; 2018: Springer.

62. Mezgec S, Korousic Seljak B. NutriNet: A Deep Learning Food and Drink Image Recognition System for Dietary Assessment. Nutrients. 2017;9(7).

63. Passler S, Wolff M, Fischer WJ. Food intake monitoring: an acoustical approach to automated food intake activity detection and classification of consumed food. Physiol Meas. 2012;33(6):1073-93.

64. Tseng P, Napier B, Garbarini L, Kaplan DL, Omenetto FG. Functional, RF-Trilayer Sensors for Tooth-Mounted, Wireless Monitoring of the Oral Cavity and Food Consumption. Adv Mater. 2018;30(18):e1703257.

65. Jia W, Li Y, Qu R, Baranowski T, Burke LE, Zhang H, et al. Automatic food detection in egocentric images using artificial intelligence technology. Public health nutrition. 2019;22(7):1168-79.

66. Xu X, Wang J, Hou L, Guo Z, Li J. A Dietary Management System Using RadioFrequency Identification Technology to Collect Information on Chinese Food Consumption: Development and Feasibility Study. JMIR Mhealth Uhealth. 2018;6(8):e166.

67. McClung HL, Ptomey LT, Shook RP, Aggarwal A, Gorczyca AM, Sazonov ES, et al. Dietary Intake and physical activity assessment: current tools, techniques, and technologies for use in adult populations. American journal of preventive medicine. 2018;55(4):e93-e104.

68. Grundy QH, Wang Z, Bero LA. Challenges in Assessing Mobile Health App Quality: A Systematic Review of Prevalent and Innovative Methods. American journal of preventive medicine. 2016;51(6):1051-9.

69. Griffiths C, Harnack L, Pereira MA. Assessment of the accuracy of nutrient calculations of five popular nutrition tracking applications. Public Health Nutr. 2018;21(8):1495-502. 
70. Six BL, Schap TE, Zhu FM, Mariappan A, Bosch M, Delp EJ, et al. Evidence-based development of a mobile telephone food record. J Am Diet Assoc. 2010;110(1):74-9.

71. Noor Hafizah Y, Ang LC, Yap F, Nurul Najwa W, Cheah WL, Ruzita AT, et al. Validity and Reliability of a Food Frequency Questionnaire (FFQ) to Assess Dietary Intake of Preschool Children. Int J Environ Res Public Health. 2019;16(23). 



\section{Chapter 2}

\section{Importance of Details in Food Descriptions in Estimating Population Nutrient Intake Distributions}

Liangzi Zhang, Anouk Geelen, Hendriek Boshuizen, José Ferreira, Marga Ocké Nutrition Journal 2019, 18(1), 17 


\begin{abstract}
:
Background: Food consumption data with much detail in food descriptions enable their use for many purposes. However, the collection and handling of such data also require huge efforts. Our aim was to improve the efficiency of data collection and handling in 24-h dietary recalls (24HRs), by identifying less important characteristics of food descriptions (facets) and assessing the impact of ignoring them on energy and nutrient intake distributions.
\end{abstract}

Methods: In the Dutch National Food Consumption Survey 2007-2010, food consumption data was collected through 24HRs using GloboDiet software in 3819 persons. Questions on each food characteristic were asked according to the applicable facets. Food consumption data were subsequently linked to the food composition database. The importance of facets for predicting energy and each of the 33 nutrients was estimated by food group, using the random forest algorithm. Then a simulation study was performed to determine the influence of the deletion of the least important facets on population nutrient intake distributions.

Results: After $35 \%$ of facet descriptors were identified as unimportant, they were deleted from the total food consumption database. The majority $(79.4 \%)$ of the percent difference between percentile estimates of the population nutrient intake distributions before and after facet deletion ranged from $0 \%$ to $1 \%$, while $20 \%$ cases ranged from $1 \%$ to $5 \%$ and $0.6 \%$ cases more than $10 \%$.

Conclusion: We conclude that our procedure was successful in identifying less important characteristics of food description for estimation of population nutrient intake distributions. This has the potential to reduce the time needed for conducting interviews and data handling. 


\section{Background}

National food consumption surveys are important policy instruments and have been carried out successively in many countries $[1,2]$. They serve many purposes, such as identification of nutrient inadequacies at the population level, risk assessment of hazardous substances, and development of dietary guidelines $[1,3]$.

In many national food consumption surveys, food consumption data are collected through 24-hour dietary recalls (24HRs) [4, 5]. This method allows the collection of abundant food consumption data, while it is less likely to alter diet behaviour and has fewer literacy requirements of the participants than food records [6, 7]. The 24HR methodology is an openended and retrospective method. Traditionally, interviewers collect information about the foods consumed during the preceding day or the previous 24 hours by triggering the participant's memory using different cues to increase the completeness of the survey [8]. This way of detail collection enables the survey to serve multiple purposes, but in the meantime increases the complexity and duration of the interview, data handling and linkage to databases $[9,10]$.

With the advent of computers, several comprehensive dietary assessment protocols have been incorporated into computer-assisted 24HR interview software and have been used in large-scale studies $[5,11,12]$. These protocols standardize the dietary data collection procedure and help the respondents recall their food intake to the maximum extent [13]. Examples include the Automated Multiple-Pass Method (AMPM), developed by the U.S. Department of Agriculture (USDA) to conduct the dietary interview for the National Health and Nutrition Examination Survey [14]. In Europe, the International Agency for Research on Cancer (IARC) has developed the menu-driven 24HR software GloboDiet (previously known as EPIC-Soft), which was validated to be used in food consumption surveys in European countries $[15,16]$.

In the multiple-pass protocol of GloboDiet, the most time-consuming step is the collection of detailed information on each consumed food (i.e. food description). Details of each food are collected through prompt windows of facets (various characteristics of a food) comparable to the probing questions in AMPM and descriptors (predefined answers on these questions). Examples of facets are fat content, cooking method, brand name, etc. Examples of descriptors are full fat, semi-skimmed, etc. [17]. Facets and descriptors standardize the procedure among different interviewers [18, 19]. In addition, the use of facets and descriptors enables characterization of the consumed foods in terms of their content of nutrients and potentially hazardous chemicals [20].

While applying a large number of facets and descriptors provides a high level of detail, it also increases the interview and data handling duration and thus the survey costs [7]. Furthermore, some food characteristics that require reading food labels (e.g. fortification) or 
knowledge about the preparation of the food (e.g. type of fat used) are difficult to answer for many of the participants $[1,21]$. Also, linkage of consumed foods to the generic food composition database is more complex given the detailed information, because more available details increases the number of unique food-descriptor combinations that need to be linked to the food composition database [10, 22, 23]. To improve the cost-effectiveness of the survey while maintaining the quality of the data, there is a need to find a balance between the level of details in the data collection and the burden laid on participants and researchers.

The aim of the current study is to evaluate facet importance in predicting nutrient contents of foods, and the impact on population nutrient intake distributions of deleting less important facets from the data collection procedure.

\section{Methods}

\section{Data collection}

In the Netherlands, food consumption of the general Dutch population is monitored in Dutch National Food Consumption Surveys (DNFCS). The data used in this study came from the DNFCS performed from 2007 to 2010 on the diet of children and adults aged 7 to 69 years. Study design, recruitment, and results have been described elsewhere [24]. Subjects were excluded if they were pregnant, lactating, institutionalized or did not speak adequate Dutch. In total, 3819 participants $(69 \%)$ were qualified and responded to the survey.

Dietary intake of participants was collected through two 24HRs on non-consecutive days with 2-6 weeks in between. The 24HRs for 2522 persons aged 16 and older were conducted by trained dieticians through telephone interviews. The 24HRs for 1297 children between 7 to 15 years old were collected by face-to-face interviews with the children and their care takers during home visits. All interviews were conducted following a same data collection and handling protocol.

During both face-to-face and telephone 24HR interview, dieticians used the multi-step computer-based interview software GloboDiet to guide the interview and to enter the data in the computer. The average time needed to complete one face-to-face $24 \mathrm{HR}$ interview was 41 minutes and 46 minutes for telephone interviews. The GloboDiet interview consists of the following five steps: 1 . Collection of the general information, 2. Listing of foods and recipes consumed throughout the day, 3. Specification of details of foods by choosing descriptors of relevant facets and consumed amounts, 4. Quality check of inaccurate input, and 5. Dietary supplement intake [15]. The collection of details in step three took about 15 minutes. IARC provided for countries that used Globodiet as their data collection software with the common facets and descriptors. The actual selection of facets and descriptors could be adjusted according to country-specific situations. For the Dutch version of the software, a total of 16 
facets with varying numbers of descriptors were selected by experienced dieticians based on knowledge of the food market and insight in the purposes for which the data were collected (Table 1).

Table 1. The list of facets and the examples of the corresponding descriptors in Globodiet for DNFCS 2007-2010.

\begin{tabular}{|c|c|c|c|}
\hline & FACET NAMES & $\begin{array}{c}\text { NUMBER OF } \\
\text { DESCRIPTORS }\end{array}$ & EXAMPLES OF DESCRIPTORS \\
\hline 1 & Source & 21 & beef, goat, pork... \\
\hline 2 & $\begin{array}{c}\text { Physical state/form } \\
\text { as quantified }\end{array}$ & 28 & liquid, reconstituted from powder, minced ... \\
\hline 3 & Cooking method & 28 & cooked, baked, barbecued... \\
\hline 4 & Preservation method & 13 & canned, frozen, dried... \\
\hline 5 & Packing medium & 22 & canned in oil, canned in water... \\
\hline 6 & $\begin{array}{l}\text { Flavoured } \\
\text { component }\end{array}$ & 37 & nuts, spices, mint... \\
\hline 7 & Sugar content & 6 & non sweetened, sweetened, sugar reduced... \\
\hline 8 & Fat content & 39 & whole, partially skimmed, skimmed... \\
\hline 9 & Type of packing & 4 & in box, in paper, in bottle... \\
\hline 10 & Food production & 12 & homemade fat used known, commercial fat used unknown... \\
\hline 11 & Enriched/fortified & 11 & vitamins, mineral components, dietary fibre... \\
\hline 12 & $\begin{array}{l}\text { Brand name } \\
(\text { yes } / \text { no })^{\mathrm{a}}\end{array}$ & 2 & yes, no \\
\hline 13 & Skin consumed & 3 & undefined, without skin, with skin \\
\hline 14 & $\begin{array}{l}\text { Visible fat } \\
\text { consumed }\end{array}$ & 3 & undefined, without visible fat, with visible fat \\
\hline 15 & Type of fat used & 2 & no fat used, choose from food list \\
\hline 16 & $\begin{array}{c}\text { Type of milk/liquid } \\
\text { used }\end{array}$ & 13 & milk, whole milk, skimmed milk... \\
\hline
\end{tabular}

a A brand name would be entered if participants chose the descriptor 'yes', entered brand names were not put in the random forest analysis in this study. 


\section{Data handling}

The total collected consumption data from all participants for the two 24HRs has 219,006 food records, with 350,369 descriptors ranging from 0 to a maximum of 8 for individual foods. This results in a number of 26,679 unique combinations of foods with descriptors. All food records were linked to 1599 most appropriate food codes in the Dutch National Food Composition Database (NEVO table 2011/3.0) by trained dieticians. NEVO 3.0 contains the energy, macro- and micronutrient contents of 2,389 food codes in total [25].

\section{Statistical analysis}

To assess the importance of the GloboDiet facets in predicting the nutrient contents of foods within a specific food group consumed in DNFCS, random forest prediction modelling was used [26]. Random forest is a prediction model that consists of a multitude of decision trees. Each tree is trained on different subsets of training data, and the remaining data (not used for the training) are used to estimate prediction error and variable importance. In our study, foods consumed by all participants in both $24 \mathrm{HRs}$ were used for predicting facet importance, the number of randomly selected variables to be considered when splitting the tree at each node was set to its default value (mtry = Total number of predictor variables/3); the number of trees for each nutrient was set at 10,000. Stratified by food group, the importance of a facet (denoted by \%IncMSE), was calculated as the percentage increase in prediction error, when data for that facet were permuted in the dataset, while keeping data for the other facets unchanged. The random forest algorithm was applied through the randomForest package in Rstudio 1.1.383.

The 24HR variables of 16 facets, food IDs (a series of numbers identifying food items) and food subgroups (elements of main food groups) were regarded as predictor variables. The detailed food group information can be found in Addition file 1. The energy and 33 macroand micronutrients were regarded as response variables and were predicted one by one with the prediction variables. Food IDs were treated as continuous variables, because it exceeds the limit of 32 levels allowed to categorical variables in the implementation of random forest. As comparable foods are numbered sequentially, treating food ID as continuous is reasonable. Facets were treated as categorical variables. Facet "Flavoured/added components" was separated into three sub-groups based on the category (nuts, sugary, savoury) of its descriptors, since the number of descriptors also exceeded the allowed 32 for categorical variables like in food IDs. The variable brand name was not included as predictor, as this consists of a free text field, yielding many unordered categories that were difficult to separate into sub-groups. Instead, we included the facet "Brand name (yes/no)" that indicated whether this brand name field was filled in or not. 
In order to facilitate the comparison of the relative importance of facets between nutrients, within each food group and each nutrient, \%IncMSEs were normalized by dividing them by the highest \%IncMSE over the facets. The maximum normalized \%IncMSE for the facet across all nutrients would be retained for each food group. After deleting facets with a max. normalized \%IncMSE lower than 0.80 in each food group, trivial effects on population nutrient intake distributions were observed, therefore a cut-off point at 1.00 was chosen for greater effects. Hence, in each food group, facets with a normalized value below 1.00 for all nutrients were considered unimportant.

\section{Simulation study}

A simulation study was conducted to evaluate whether the distributions of population nutrient intake change significantly when the less important facets would not have been asked for during the 24HRs. The average nutrient intake calculated from two 24 HRs of each participant was used in the simulation study for estimating population nutrient intake distributions.

The simulation study consisted of two steps. Firstly, food-descriptor combinations with one or more facets which were considered unimportant were identified in the dataset with unique food-descriptor combinations. These food-descriptor combinations were relinked to the national food composition database NEVO considering only important facets. As illustrated in Figure 1, a NEVO code reassignment protocol was developed to identify NEVO codes of the most similar food-descriptor combinations with the combination of facets that needed to be relinked considering only important facets. For foods-descriptor combinations in the dataset with same food IDs, combinations received a positive score for each identical pair of descriptors (equal to the maximum normalized \%IncMSEs) and a penalty for descriptors that were different (equal to the negative maximum normalized \%IncMSEs). The scores were summed and the NEVO code of the food-descriptor combination with the highest score was assigned to the combination that needed to be relinked. In case there were more than one NEVO codes with the same highest score, or when no descriptors were left for a food, the NEVO code of a food-descriptor combination with a higher consumed quantity would be selected. In case the consumed quantities were also the same (occurred in 38 cases), the decision on NEVO code selection was made by a researcher.

Secondly, the energy and nutrient contents for 100 grams of foods in NEVO were multiplied with the quantities consumed in DNFCS 2007-2010, summarised by person by day and averaged over two days in both the dataset with original linkage to the NEVO database and the newly linked dataset. All results were weighted for small deviances in sociodemographic characteristics (age, sex, region, degree of urbanisation and educational level), day of the week and season of data collection, in order to give results that are representative for the Dutch population and representative for all days of the week and all seasons. The mean, 
median, $5^{\text {th }}, 25^{\text {th }}, 75^{\text {th }}, 95^{\text {th }}$ percentile and the percent differences of consumption per nutrient between the original and newly linked dataset were calculated for the total population and stratified by gender and age group (7-18 years old and 19-69 years old). The population nutrient intake distributions were conducted using the SAS 9.4 and the percent difference between the original and newly linked dataset were calculated using Excel 2016 software.

NEVO Code Reassignment Protocol

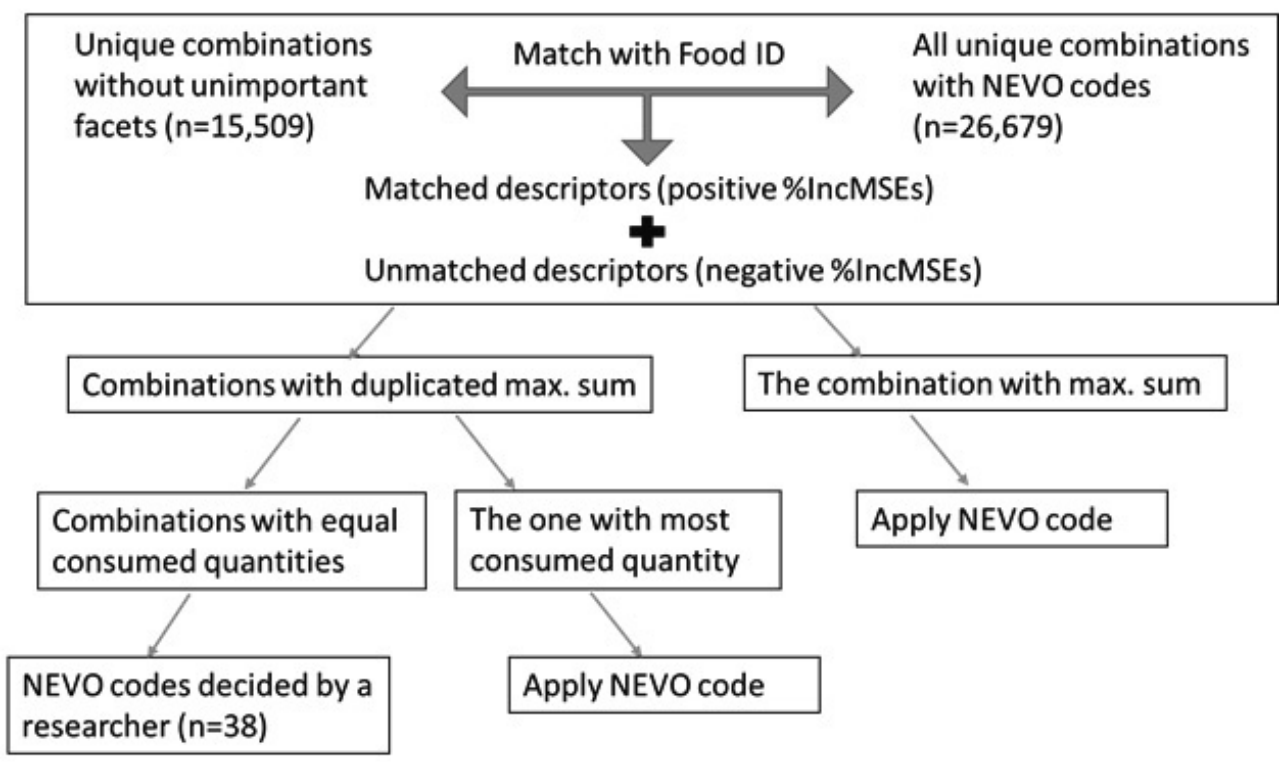

Figure 1. Flow chart of the NEVO code Reassignment Protocol. A NEVO code was assigned to each relinking combination according to the NEVO codes of the same food with the most similar descriptor combinations that have been linked by dieticians during the survey period. The combinations received a positive score for each identical pair of descriptors (equal to the maximum normalized \%IncMSEs) and a penalty for descriptors that were different (equal to the negative maximum normalized \%IncMSEs). The scores were summed, and the NEVO code of the food-descriptor combination with the highest score was assigned to the combination that needed to be relinked. In case there were more than one NEVO codes with the same highest score, or when no descriptors were left for a food item, the NEVO code of a food-descriptor combination with a higher consumed quantity would be selected. In case the consumed quantities were also the same (occurred in 38 cases), a researcher decided on NEVO code selection. 


\section{Results}

Table 2 shows the normalized maximum importance (\%IncMSEs) of each of 16 facets in predicting the nutrient contents of food items within each of 17 food groups using the cutoff point of 1.00, whereas results for the cut-off of 0.80 are shown in Additional File 2. Using the cut-off level of 0.80 , a total of 50 out of 112 facets across food groups were considered unimportant. When the unimportant facets were deleted from the total food consumption database, $22 \%$ of the 350,369 facet descriptors were omitted. The majority of the percent difference between percentile estimates of the population nutrient intake distributions before and after facet deletion ranged from $0 \%$ to $1 \%$, while only $2 \%$ cases ranged from $1 \%$ to $5 \%$.

Across food groups, a total of 64 out of 112 facets fell below the cut-off point at 1.00, and have been deleted from the corresponding food groups in the simulation study. In the food groups 'Fats and oils' and 'Alcoholic beverages', no facets were unimportant, whereas all facets were unimportant for 'Cakes and sweet biscuits'. The food group 'Miscellaneous' has the largest amount of unimportant facets than the rest of the food groups. In the 'Meat' group, most facets had zero effect in predicting food groups, including 'Source', 'Packing medium', 'Fat content', 'Brand name (yes/no)', 'skin consumed, and 'visible fat consumed'.

From the perspective of the facets, 'Brand name (yes/no)' and 'Packing medium' were unimportant for the most of the food groups (10 and 7 food groups, respectively). The number of deletions ranged from 1 to 5 times for the rest of the facets. 'Source' and 'Visible fat consumed' were unimportant for all the food groups for which they are relevant ( 3 and 1 food groups, respectively). On the other hand, 'Physical state' and 'Cooking method' were strong predictors (importance of 1.00) for the largest number of food groups. Facet 'Type of packing' was only available for food group 'Fats and oils' and was a strong predictor for that food group. Despite that 'Brand name (yes/no)' was unimportant for most of the food groups, it was a strong predictor for food group 'Cereals', 'Fats and oils', 'Alcoholic' and 'Nonalcoholic beverages'. Full results of the facet importance for each nutrient in each food group can be found in Additional File 3.

In the original total food consumption database, $35 \%(121,015$ out of 350,369$)$ of the total number of descriptors used were identified as unimportant, which has resulted a NEVO code change of $11 \%(2,923$ out of 26,679$)$ of the combinations in the unique food dataset and $3.7 \%$ $(8,196$ out of 219,006$)$ of the combinations in the total food consumption dataset.

After the NEVO codes had been reassigned, the population means and percentiles of two days' average energy and nutrient intakes in DNFCS 2007-2010 were calculated, as well as the percent difference between them. Table 3 shows the results of energy and ten nutrients that were mostly found in nutrition facts label. The results of all nutrients can be found in Additional File 4. The majority $(79.4 \%)$ of the percent difference between distribution 
percentiles before and after facet deletion ranged from $0 \%$ to $1 \%$, while $20 \%$ cases ranged from $1 \%$ to $5 \%$ and $0.6 \%$ cases more than $10 \%$. Percent difference larger than $1 \%$ were mainly found in vitamins. Differences more than $10 \%$ appeared mostly in vitamins for 7-18 year olds and in the extreme percentiles P5 and P95. Some of the differences that were larger than $10 \%$ were very small as absolute difference. For example, the largest differences of $14.1 \%$ was for the P95 of vitamin B6; but the absolute difference of the two scenarios was $0.5 \mathrm{mg}$ (rounded to $\mathrm{mg}$ ). No general patterns were found on nutrient over- and underestimation after facet deletion for most nutrients. However, less vitamin $\mathrm{C}$ was found in each percentile after facet deletion for all age groups, whereas higher amounts of vitamin B group were found after facet deletion. 


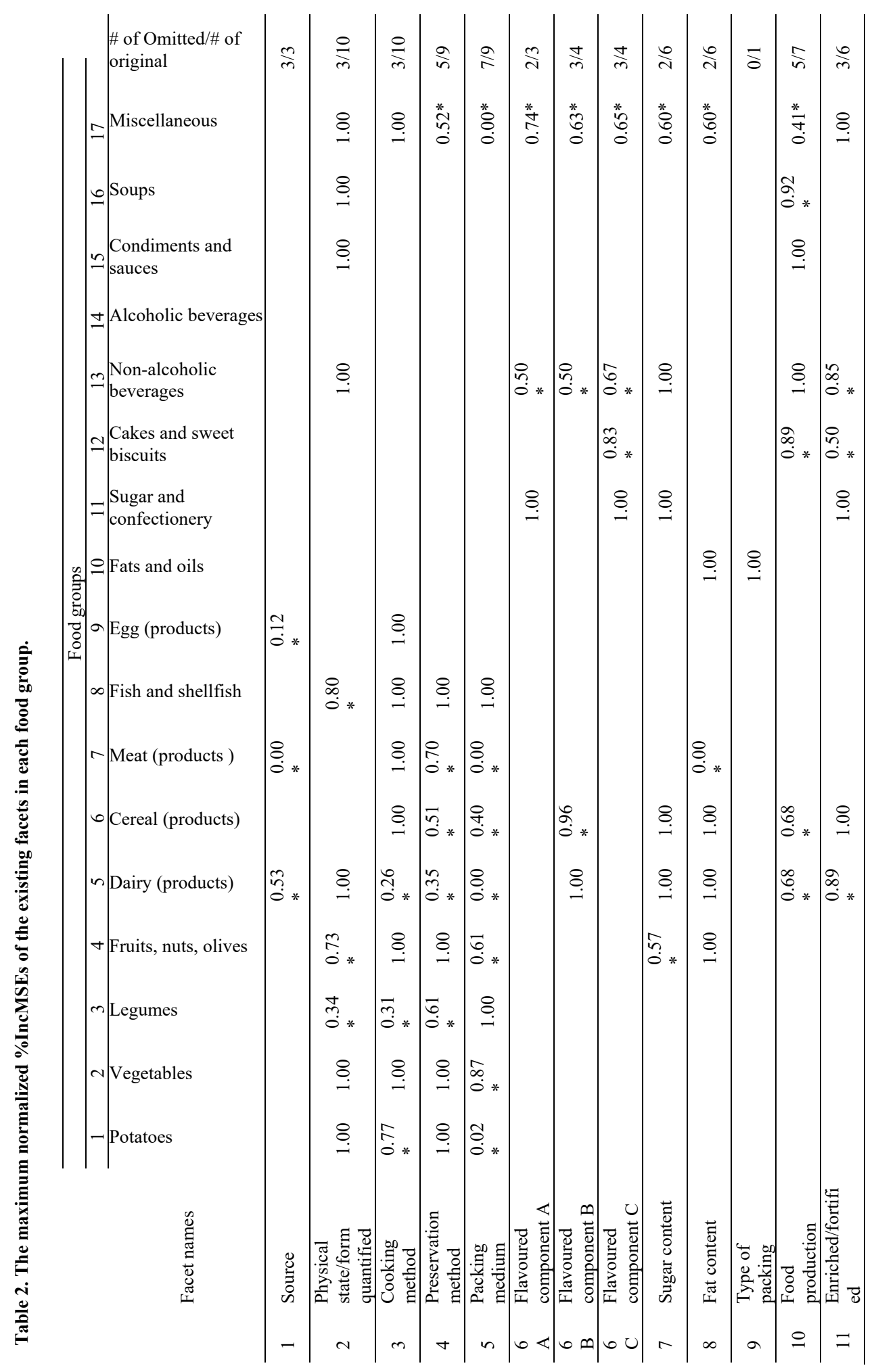




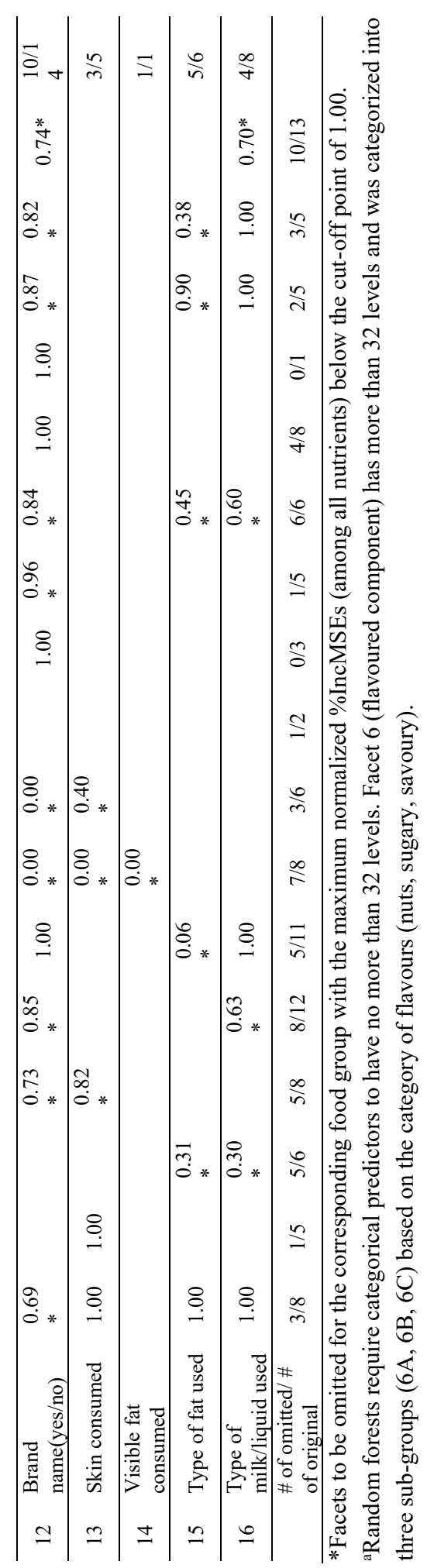




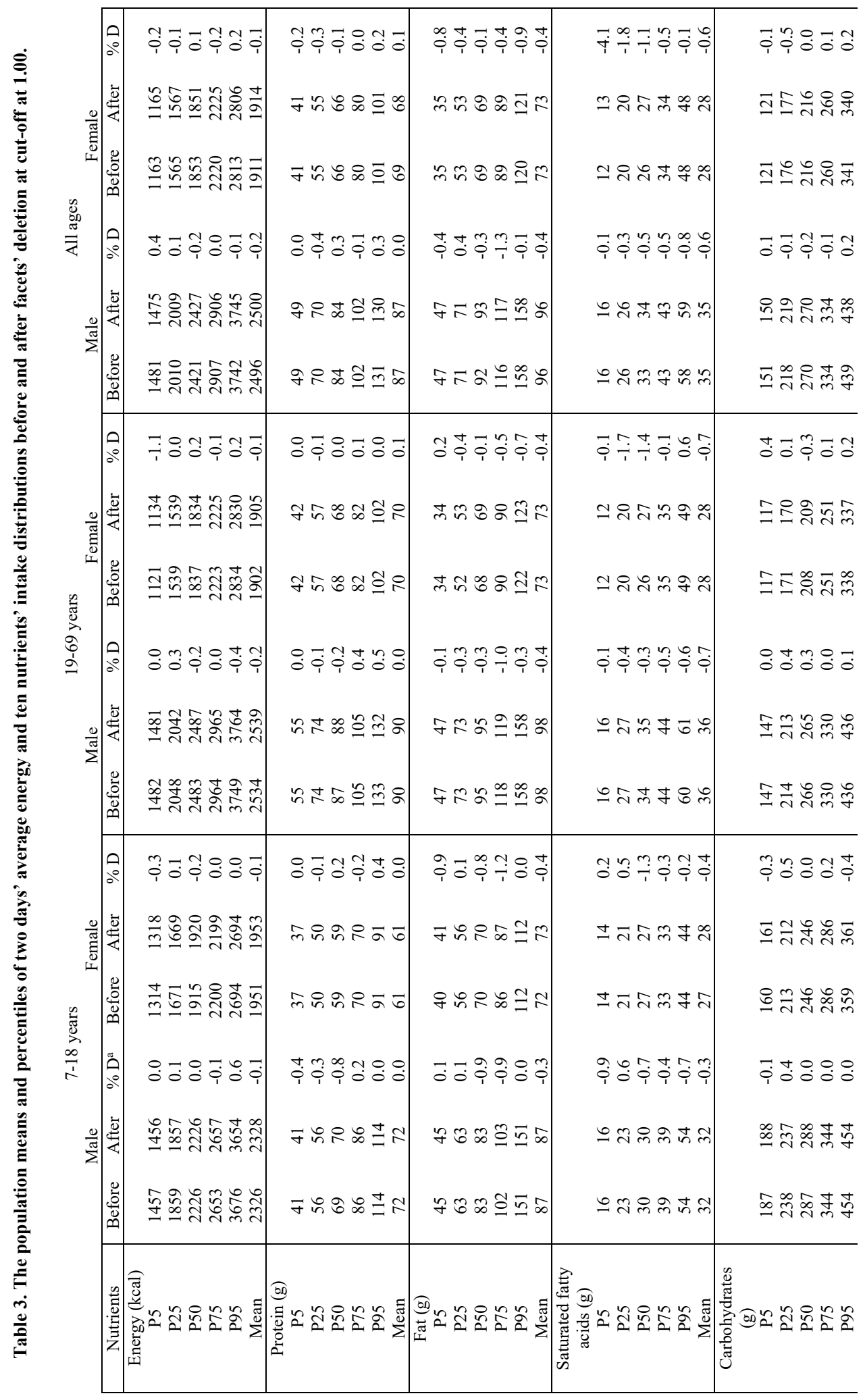




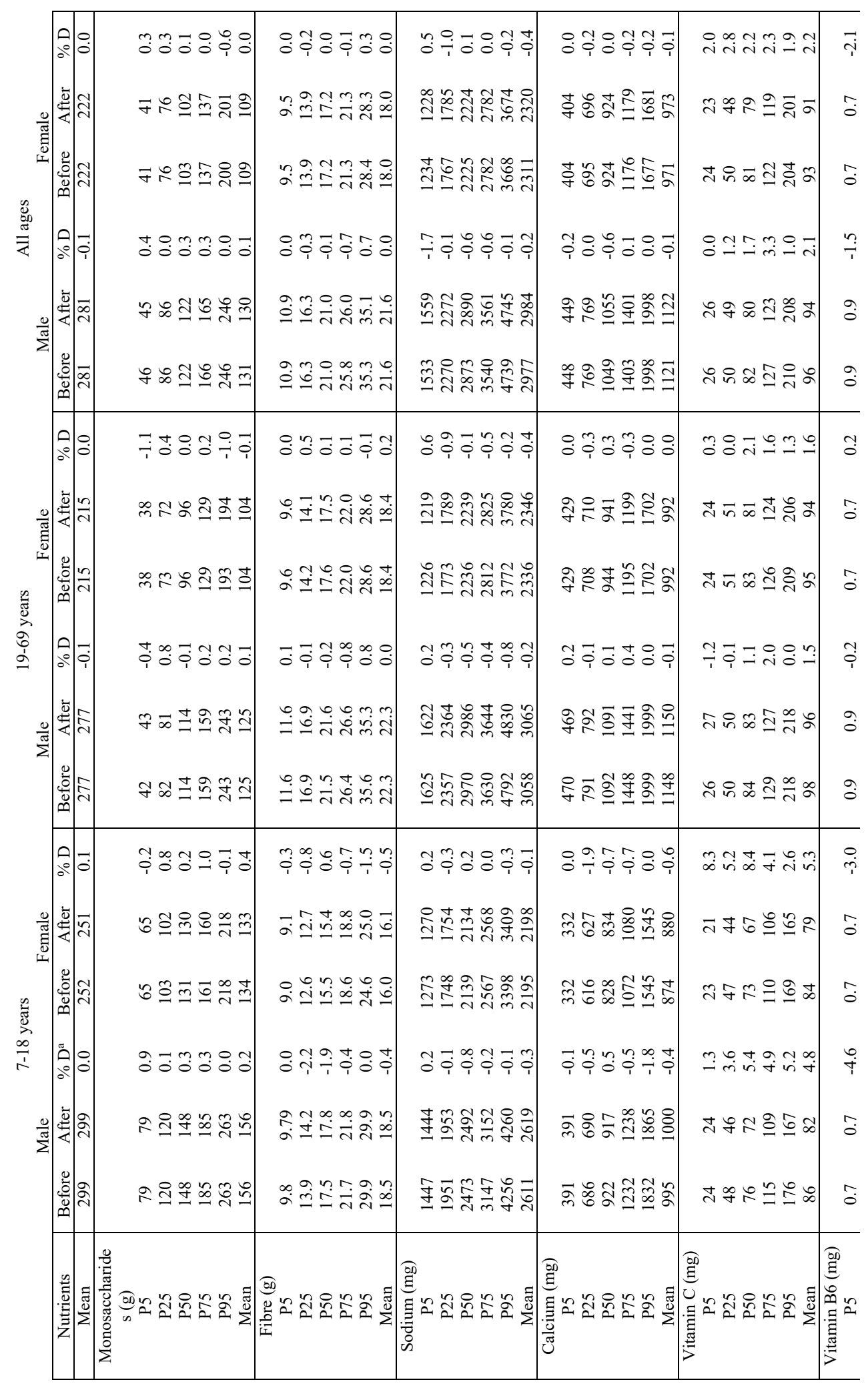




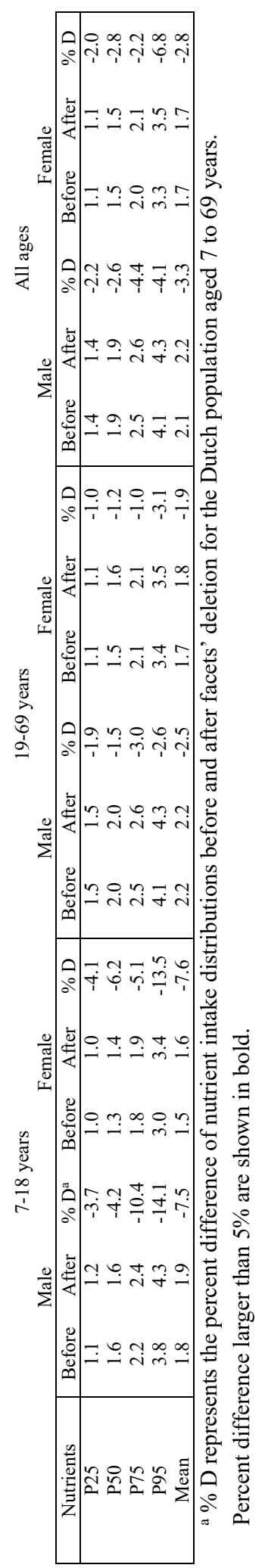




\section{Discussion}

From the perspective of enhancing the efficiency of data collection and handling of GloboDiet 24HRs, we explored the option of deleting less important characteristics (facets) of food descriptions from the interview. At the food group level, the importance of each facet in predicting nutrient contents in foods was determined by the random forest algorithm. When the $35 \%$ least predictive facets were deleted from the dataset of the Dutch national food consumption survey 2007-2010, the difference between recalculated and originally calculated population nutrient intake distributions was small for the majority of the nutrients.

There are several possible explanations for certain facets to be less or more predictive in certain food groups. One reason for less predictive facets is that some facets were only applicable to few food items in certain food groups, and those food items were rarely consumed. An example of this is the facet 'Enriched/fortified' in the food group 'Cakes and sweet biscuits'. A second reason is a lack of variation in the chosen descriptors within a facet. An example of this is the facet 'source' in dairy products since in the Netherlands cow milk is the basis for the majority of the consumed dairy products. Another possible explanation for the less predictive facets is the use of a generic food composition database NEVO [27]. Some facets might have been important for predicting true nutrient levels but not for averaged nutrient levels of a generic food. An example of this could be brand name for predicting salt levels of industrially processed foods [28]. In contrast, some facets showed strong predictive power in estimating nutrient contents in certain food groups. The facet 'Type of packing' was predicts strongly for the 'Fats and oils' group, because the type of packing materials distinguishes solid from liquid fat, which results in different nutrient contents, specifically for fat content. Similarly, as can be expected from a nutrition point of view, facet 'Physical state', 'Sugar' and 'Fat content' were strong predictors for most of their allocated food groups, except for unprocessed products (e.g. fruit, meat, and fish).

In terms of comparing nutrient intake distributions before and after the facets had been deleted, difference of less than $10 \%$ was found for most nutrients. This could be explained by the fact that $96.3 \%$ of the combinations were relinked to a same food code in the food composition database. Apparently, the food name and remaining facets provided sufficient information to link to the same food item in the Dutch National Food Composition Database. For those combinations with deleted facets that were linked to different food codes in the food composition database, the difference in nutrient contents of the original and alternative food codes may have been small, or the foods were consumed by few persons or in small amounts and therefore did not influence population nutrient intake distributions substantially. A similar finding was observed in a study that investigated the effect of a concise versus an extensive food list in a self-administered web-based 24HR tool. They observed that the differences between population nutrient intakes assessed by two methods were less than $6 \%$ 
[29], which is consistent with our study that the majority of the differences fell below $5 \%$ before and after facet deletion.

Specifically, a large decrease in the amount of vitamin C was found for children in our study, the reason was speculated to be the deletion of the facet 'Enriched/fortified' in the food group 'Non-alcoholic beverages'. According to the report of 2007-2010 survey, 'Non-alcoholic beverages' and 'Meat and meat products' together, contribute for one third to the total vitamin $\mathrm{C}$ intake partly due to food fortification and processing [24]. Hence, beverages with fortification were linked to NEVO codes for products without fortification and resulted a lower vitamin $\mathrm{C}$ content. On the other hand, a large increase in the amount of vitamin $\mathrm{B}$ group was found for children. A possible explanation would be the deletion of 'Flavoured component' in the food group of 'Cereal', which may have caused the linkage of NEVO codes high in vitamin B contents (i.e. whole wheat cereals) with flavoured regular cereals. A closer investigation should be conducted before deleting facets in the real setting.

To our knowledge, this is the first study investigating the impact of reducing food descriptions in interview-based 24HRs for the estimation of population nutrient intake distributions. A strength of our approach is that both the identification of facets to be deleted as well as assessing its impact was data driven. Until now decisions on the facets that were included in the 24HR interview of DNFCS were based on expert-judgment. Another strength is the use of the random forest for the identification of unimportant facets. This prediction model is more efficient in large datasets, has a lower risk of overfitting and is better in dealing with correlated predictors than multiple linear regression [30]. However, the applied random forest implementation only allows nominal variables with a limited number of levels as predictors. Therefore, the nominal variable "food ID" was treated as a continuous variable, and the importance of the information on the full brand and product name of each food could not be evaluated. In addition, importance of the facet "Cooking method" could not fully be assessed, since the added fat in case of frying was not included in the nutrient content of the food, but became a separate food item in the food consumption database. Another limitation of our study was the use of a semi-automated protocol of reassigning a different NEVO code to combinations with deleted facets rather than applying the original approach of 'manual' linkage by dieticians. Manual matching, however, would only have further decreased the effect of facet deletion, so we do not think our conclusions would have been different. Finally, the impact of facet reduction on respondents' answers during the food description part of the interview was not assessed. Although a face-to-face or telephone $24 \mathrm{HR}$ interview has generally smaller self-reporting error than other methods, measurement error is likely to be present (i.e. rely on memory, underreporting) [6]. However, we assume that the effect of facet reduction on self-reporting error will be small. 
The scope of our analyses focussed on the importance of facets on nutrient intake distribution. In addition, other aspects are also important in deciding which facets to be deleted. One example is that the facet 'Physical state' is important during the interview to determine the options for quantifying the consumed foods, e.g. coffee powder is quantified differently than coffee as a beverage. Moreover, the effects of facet omission on the estimation of exposure to food chemicals that are potentially hazardous should be considered for the DNFCS. In principle the procedure described in this manuscript can also be applied to evaluate facet importance for food chemical distributions.

The objective of looking at the reduction in food characteristics was to enhance efficiency in conducting future surveys. Less extensive food description would result in a shorter time needed for the 24HR interview and to match the reported foods to the food composition database. The time needed to complete the facet collection procedure of a 44 minutes $24 \mathrm{HR}$ interview was estimated to be 15 minutes. Without $35 \%$ of the unimportant facets, the time saved for one interview would on average be 5 minutes. In a survey with 3819 participants that are interviewed twice, a total of 637 hours would be saved. In terms of linking reported foods to the food composition database, time would be saved due to a reduction in unique food-descriptor combinations. The average time needed to link a combination to the food composition table was estimated to be 5 to 10 minutes. After deleting less important facets from the unique food-descriptor combination list, the number of unique combinations reduced with 3534 (from 26679 to 23145). In the data handling of DNFCS, only new fooddescriptor combinations needed to be linked to the food composition database manually. Therefore, around 442 hours would be saved for data handling to link each unique fooddescriptor to the food composition code. To sum up, we estimated that around 1079 hours would be saved for both data collection and handling if facet deletion would be applied.

The current study focused on reducing the number of facets as a potential efficiency measure for a national food consumption survey. For taking final decisions, advantages and disadvantages of alternative efficiency options could be taken into consideration. One alternative is to use 24-h dietary recall software to guide the interviews in which the food list is directly related to the foods in the national food composition database [9, 31]. The reason why GloboDiet did not choose for this option was to give flexibility for new foods that enter the food market (and are not included in food composition databases yet), to standardize food description across different countries that use the same software, and to be able to collect characteristics of food relevant for other purposes than nutrient intake estimations [17]. A more cost-efficient alternative regarding dietary assessment is to use self-administered methods. However, the accuracy and reliability of those tools needs to be further evaluated to be applied in large-scale surveys, due to self-reporting errors, and various levels of acceptance by different age-groups [32]. Thirdly, the matching of food consumption and food composition data could be made more efficient through automatic or semi-automatic 
linkages. In this study, decisions on NEVO code reassignment for food-descriptor combinations were made based on a simple algorithm with the results of the random forest algorithm. For matching future food consumption data automatically or semi-automatically, random forest prediction models using available previously matched food consumption and food composition data as training dataset could be developed. Two studies have developed a semi-automatic food matching technique using machine-learning and a natural language processing approach. Both studies have tested the effectiveness of the approach as compared to manually link the food items to the food codes by experts, and have shown the approaches to be effective [33,34]. These procedures need further validation before they can be implemented in a large-scale survey.

\section{Conclusion}

In conclusion, the data-driven procedure that combined random forest prediction with a simulation study was successful in identifying less important characteristics of food description. When deleting those less important characteristics, there was little impact on the estimation of the distributions of population nutrient intake for most nutrients, thus yielding a promising approach for saving labour and costs. 


\section{Reference}

1. Ocke MC, Van Rossum CTM, de Boer EJ. Dietary Surveys: National Food Intake. In: Caballero B, Finglas P, Toldrà F, editors. The Encyclopedia of Food and Health. 2. Oxford: Academic Press; 2016. p. 432-8.

2. Rippin H, Hutchinson J, Evans C, Jewell J, Breda J, Cade J. National nutrition surveys in Europe: a review on the current status in the 53 countries of the WHO European region. Food \& Nutrition Research [Internet]. 2018;62. https://doi.org/10.29219/fnr.v62.1362

3. Ahluwalia N, Dwyer J, Terry A, Moshfegh A, Johnson C. Update on NHANES Dietary Data: Focus on Collection, Release, Analytical Considerations, and Uses to Inform Public Policy. Advances in nutrition. 2016;7(1):121-34. https://doi.org/10.3945/an.115.009258

4. de Boer EJ, Slimani N, van 't Veer P, Boeing H, Feinberg M, Leclercq C, et al. Rationale and methods of the European Food Consumption Validation (EFCOVAL) Project. Eur J Clin Nutr. 2011;65 Suppl 1:S1-4. https://doi.org/10.1038/ejcn.2011.82

5. De Keyzer W, Bracke T, McNaughton SA, Parnell W, Moshfegh AJ, Pereira RA, et al. Cross-continental comparison of national food consumption survey methods--a narrative review. Nutrients. 2015;7(5):3587-620. https://doi.org/10.3390/nu7053587

6. EFSA. General principles for the collection of national food consumption data in the view of a pan-European dietary survey. EFSA Journal. 2009;7(12):1435. https://doi.org/10.2903/j.efsa.2009.1435

7. Grandjean AC. Dietary intake data collection: challenges and limitations. Nutrition reviews. 2012;70 Suppl 2:S101-4. https://doi.org/10.1111/j.1753-4887.2012.00545.x

8. Carriquiry AL. Understanding and Assessing Nutrition. Annu Rev Stat Appl. 2017;4:123-46. https://doi.org/10.1146/annurev-statistics-041715-033615

9. Zimmerman TP, Hull SG, McNutt S, Mittl B, Islam N, Guenther PM, et al. Challenges in converting an interviewer-administered food probe database to selfadministration in the National Cancer Institute Automated Self-administered 24-Hour Recall (ASA24). Journal of food composition and analysis : an official publication of the United Nations University, International Network of Food Data Systems. 2009;22(Suppl 1):S48-S51. https://doi.org/10.1016/j.jfca.2009.02.003

10. Ocke MC, Slimani N, Brants H, Buurma-Rethans E, Casagrande C, Nicolas G, et al. Potential and requirements for a standardized pan-European food consumption survey using the EPIC-Soft software. Eur J Clin Nutr. 2011;65:S48-S57.

https://doi.org/10.1038/ejen.2011.87

11. Timon CM, van den Barg R, Blain RJ, Kehoe L, Evans K, Walton J, et al. A review of the design and validation of web- and computer-based 24-h dietary recall tools. Nutrition research reviews. 2016;29(2):268-80. https://doi.org/10.1017/S0954422416000172

12. Raper N, Perloff B, Ingwersen L, Steinfeldt L, Anand J. An overview of USDA's dietary intake data system. J Food Compos Anal. 2004;17(3-4):545-55. https://doi.org/10.1016/j.jfca.2004.02.013 
13. Shim J-S, Oh K, Kim HC. Dietary assessment methods in epidemiologic studies. Epidemiology and Health. 2014;36:e2014009. https://doi.org/10.4178/epih/e2014009

14. Moshfegh AJ, Rhodes DG, Baer DJ, Murayi T, Clemens JC, Rumpler WV, et al. The US Department of Agriculture Automated Multiple-Pass Method reduces bias in the collection of energy intakes. American Journal of Clinical Nutrition. 2008;88(2):32432. https://doi.org/10.1093/ajcn/88.2.324

15. Slimani N, Ferrari P, Ocke M, Welch A, Boeing H, Liere M, et al. Standardization of the 24-hour diet recall calibration method used in the european prospective investigation into cancer and nutrition (EPIC): general concepts and preliminary results. Eur J Clin Nutr. 2000;54(12):900-17.

16. de Boer EJ, Slimani N, van 't Veer P, Boeing H, Feinberg M, Leclercq C, et al. The European Food Consumption Validation Project: conclusions and recommendations. Eur J Clin Nutr. 2011;65 Suppl 1:S102-7. https://doi.org/10.1038/ejcn.2011.94

17. Slimani N, Casagrande C, Nicolas G, Freisling H, Huybrechts I, Ocke MC, et al. The standardized computerized 24-h dietary recall method EPIC-Soft adapted for panEuropean dietary monitoring. Eur J Clin Nutr. 2011;65 Suppl 1:S5-15. https://doi.org/10.1038/ejen.2011.83

18. Slimani N, Deharveng G, Charrondiere RU, van Kappel AL, Ocke MC, Welch A, et al. Structure of the standardized computerized 24-h diet recall interview used as reference method in the 22 centers participating in the EPIC project. European Prospective Investigation into Cancer and Nutrition. Computer methods and programs in biomedicine. 1999;58(3):251-66.

19. Steinfeldt L, Anand J, Murayi T. Food Reporting Patterns in the USDA Automated Multiple-Pass Method. Procedia Food Science. 2013;2:145-56. https://doi.org/10.1016/j.profoo.2013.04.022

20. Bakker M, Fransen H, Ocke M, Slob W. Evaluation of the Dutch National Food Consumption Survey with respect to dietary exposure assessment of chemical substances. 2010.

21. Huybrechts I, Geelen A, de Vries JH, Casagrande C, Nicolas G, De Keyzer W, et al. Respondents' evaluation of the 24-h dietary recall method (EPIC-Soft) in the EFCOVAL Project. Eur J Clin Nutr. 2011;65 Suppl 1:S29-37. https://doi.org/10.1038/ejen.2011.85

22. Zimmerman TP, Potischman N, Douglass D, Dixit-Joshi S, Kirkpatrick SI, Subar AF, et al. The Effect of Editing Open-Ended Text Responses on Nutrient and Food Group Estimates from the Automated Self-Administered 24-Hour Dietary Recall (ASA24). Procedia Food Science. 2015;4:160-72. http://dx.doi.org/10.1016/j.profoo.2015.06.021

23. Stadlmayr B, Wijesinha-Bettoni R, Haytowitz D, Rittenschober D, Cunningham J, Sobolewski R, et al. FAO/INFOODS Guidelines on Food Matching version 1.2. Rome: FAO; 2012. 
24. van Rossum CTM, Fransen HP, Verkaik-Kloosterman J, Buurma EM, Ocke MC. Dutch National Food Consumption Survey 2007-2010: Diet of children and adults aged 7 to 69 years. Bilthoven: RIVM; 2011. Report No.: RIVM-report 350070006.

25. NEVO-table [Internet]. Nutrition Center. 2011. https://nevo-online.rivm.nl/. Accessed 23 July 2018.

26. Breiman L. Random Forests. Machine Learning. 2001;45(1):5-32. https://doi.org/10.1023/a:1010933404324

27. NEVO-online 2016: background information. In: Westenbrink S, Vliet MJ-vd, editors. Bilthoven: RIVM; 2016.

28. Temme EHM, Hendriksen MAH, Milder IEJ, Toxopeus IB, Westenbrink S, Brants HAM, et al. Salt Reductions in Some Foods in The Netherlands: Monitoring of Food Composition and Salt Intake. Nutrients. 2017;9(7):791. https://doi.org/10.3390/nu9070791

29. Evans K, Hennessy Á, Walton J, Timon C, Gibney E, Flynn A. Development and evaluation of a concise food list for use in a web-based 24-h dietary recall tool. Journal of Nutritional Science. 2017;6:e46. https://doi.org/10.1017/jns.2017.49

30. Epifanio I. Intervention in prediction measure: a new approach to assessing variable importance for random forests. BMC Bioinformatics. 2017;18:230. 10.1186/s12859017-1650-8

31. Gavrieli A, Naska A, Konstantinidi C, Berry R, Roe M, Harvey L, et al. Dietary Monitoring Tools for Risk Assessment. EFSA Supporting Publications [Internet]. 2014 01; 11(5). Available from:

http://onlinelibrary.wiley.com/doi/10.2903/sp.efsa.2014.EN-607/abstract.

32. Conrad J, Nothlings U. Innovative approaches to estimate individual usual dietary intake in large-scale epidemiological studies. P Nutr Soc. 2017;76(3):213-9. https://doi.org/10.1017/S0029665116003025

33. Koroušić Seljak B, Korošec P, Eftimov T, Ocke M, van der Laan J, Roe M, et al. Identification of Requirements for Computer-Supported Matching of Food Consumption Data with Food Composition Data. Nutrients. 2018;10(4):433. https://doi.org/10.3390/nu10040433

34. Eftimov T, Korosec P, Seljak BK. StandFood: Standardization of Foods Using a Semi-Automatic System for Classifying and Describing Foods According to FoodEx2. Nutrients. 2017;9(6). https://doi.org/10.3390/nu9060542 
Additional File 1. Dutch National Food Consumption Survey 2007-2010 EPIC-Soft group classification.

\begin{tabular}{|c|c|c|}
\hline & Food Groups & Food sub-groups \\
\hline \multirow[t]{2}{*}{1} & Potatoes and other tubers & Unclassified, mixed and other tubers \\
\hline & & Potatoes \\
\hline \multirow[t]{9}{*}{2} & Vegetables & Unclassified, mixed salad/vegetables \\
\hline & & Leafy vegetables \\
\hline & & Fruiting vegetables \\
\hline & & Root vegetables \\
\hline & & Cabbages \\
\hline & & Mushrooms \\
\hline & & Grain and pod vegetables \\
\hline & & Leek, onion, garlic \\
\hline & & Stalk vegetables, sprouts \\
\hline 3 & Legumes & Legumes \\
\hline \multirow[t]{5}{*}{4} & Fruits, nuts and seeds, olives & Fruits \\
\hline & & Fruit compote \\
\hline & & Nuts, peanuts, seeds \\
\hline & & Peanut butter, nut/seeds spread \\
\hline & & Olives \\
\hline \multirow[t]{10}{*}{5} & Dairy products and substitutes & Non fermented milk and milk beverages \\
\hline & & Fermented milk, milk beverages and yogurt \\
\hline & & Milk substitutes \\
\hline & & Yoghurt \\
\hline & & Fromage blanc, petits suisses \\
\hline & & Cheeses (including spread cheeses) \\
\hline & & Unclassified creams \\
\hline & & Dairy creams and creamers \\
\hline & & Nondairy creams and creamers \\
\hline & & Ice cream \\
\hline \multirow[t]{6}{*}{6} & Cereals and cereal products & Flours, starches, flakes, semolina \\
\hline & & Pasta, rice, other grain \\
\hline & & Bread \\
\hline & & Crispbread, rusks \\
\hline & & Breakfast cereals \\
\hline & & Dough and pastry \\
\hline
\end{tabular}




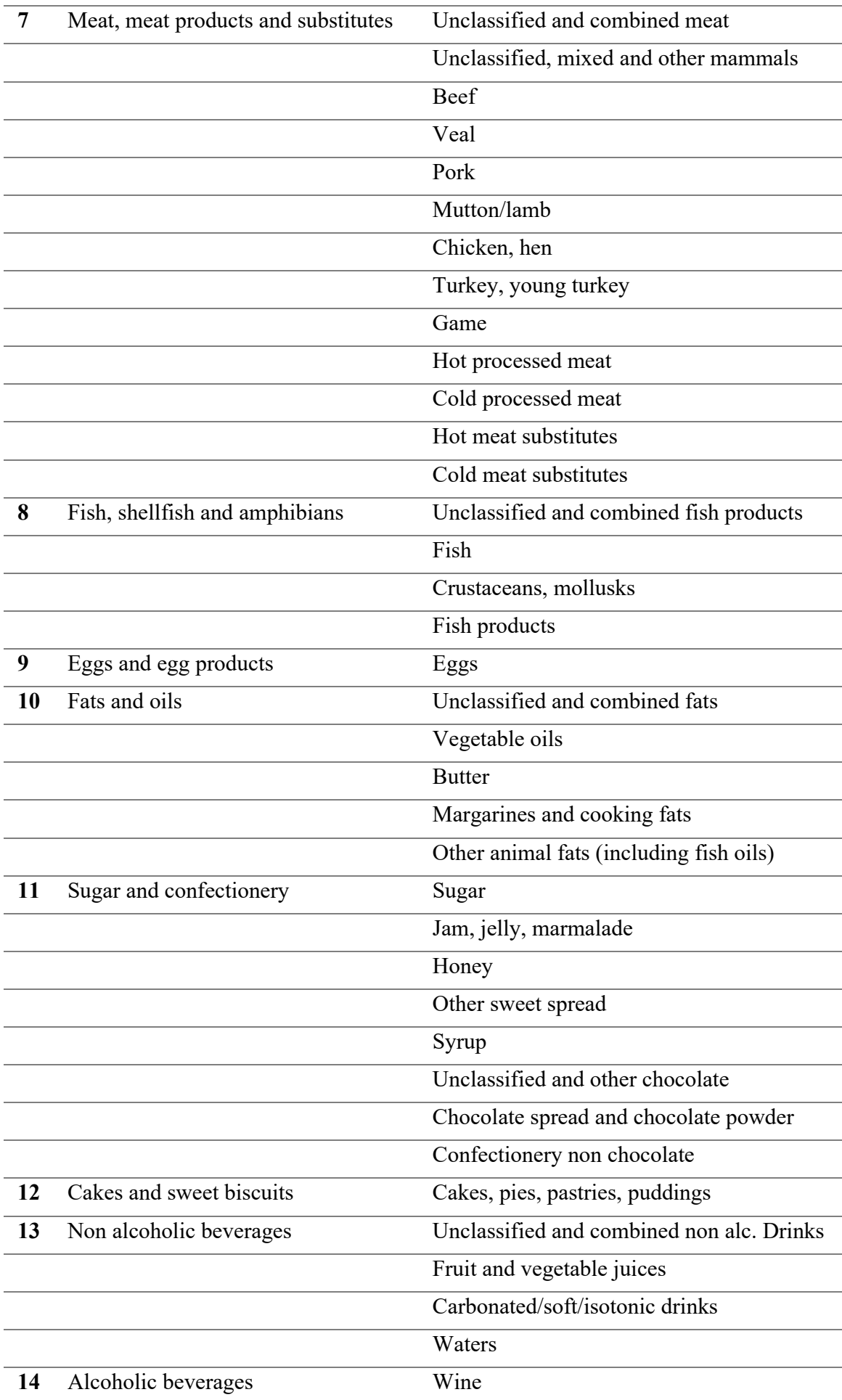




\begin{tabular}{lll}
\hline & Beer \\
\hline & Spirits \\
\hline & Liqueurs \\
\hline $\mathbf{1 5}$ & Condiments, spices, sauces and yeast & Cnclassified or combined condiments \\
\hline & Other and mixed sauces \\
\hline & Tomato sauces \\
\hline & & Dressing sauces, mayonnaises and similar \\
\hline & Mayonnaise based spreads \\
\hline & Soups and stocks & Spices, herbs and flavourings \\
\hline $\mathbf{1 6}$ & Miscellaneous & Unclassified and combined condiments \\
\hline & & Soups \\
\hline & & Stocks \\
\hline & & Artificial sweeteners \\
\hline & & Meal substitutes \\
\hline
\end{tabular}




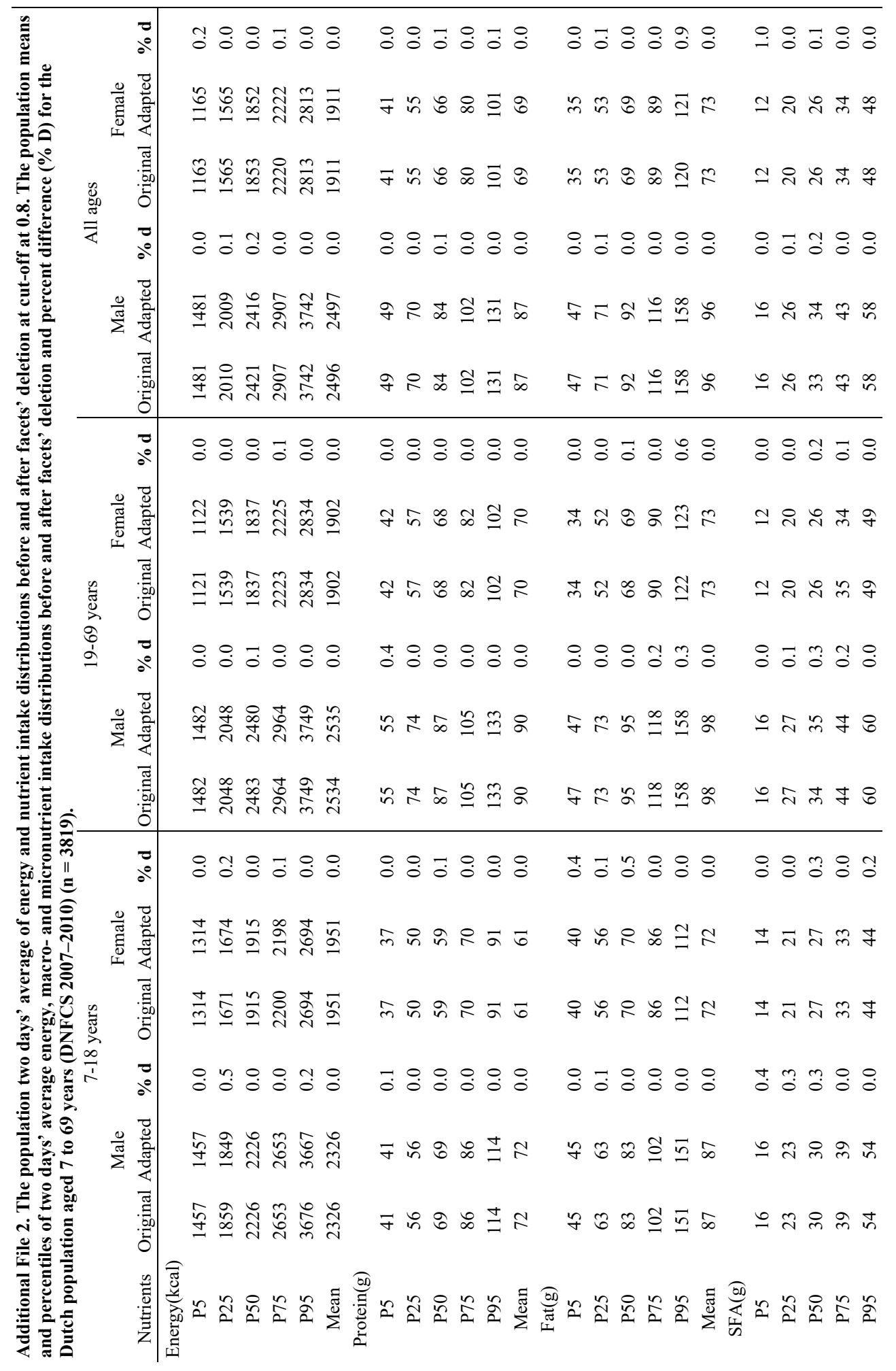




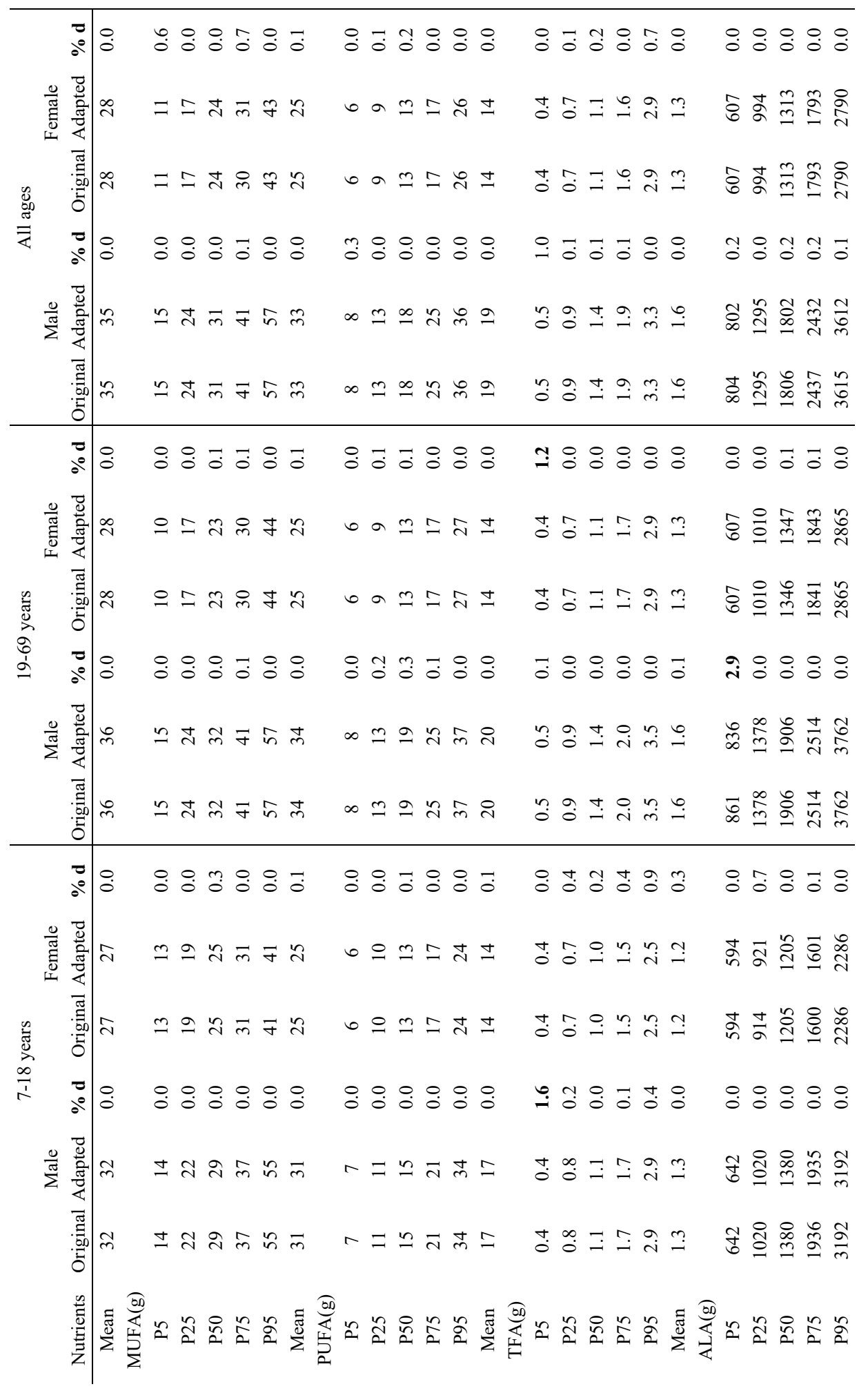




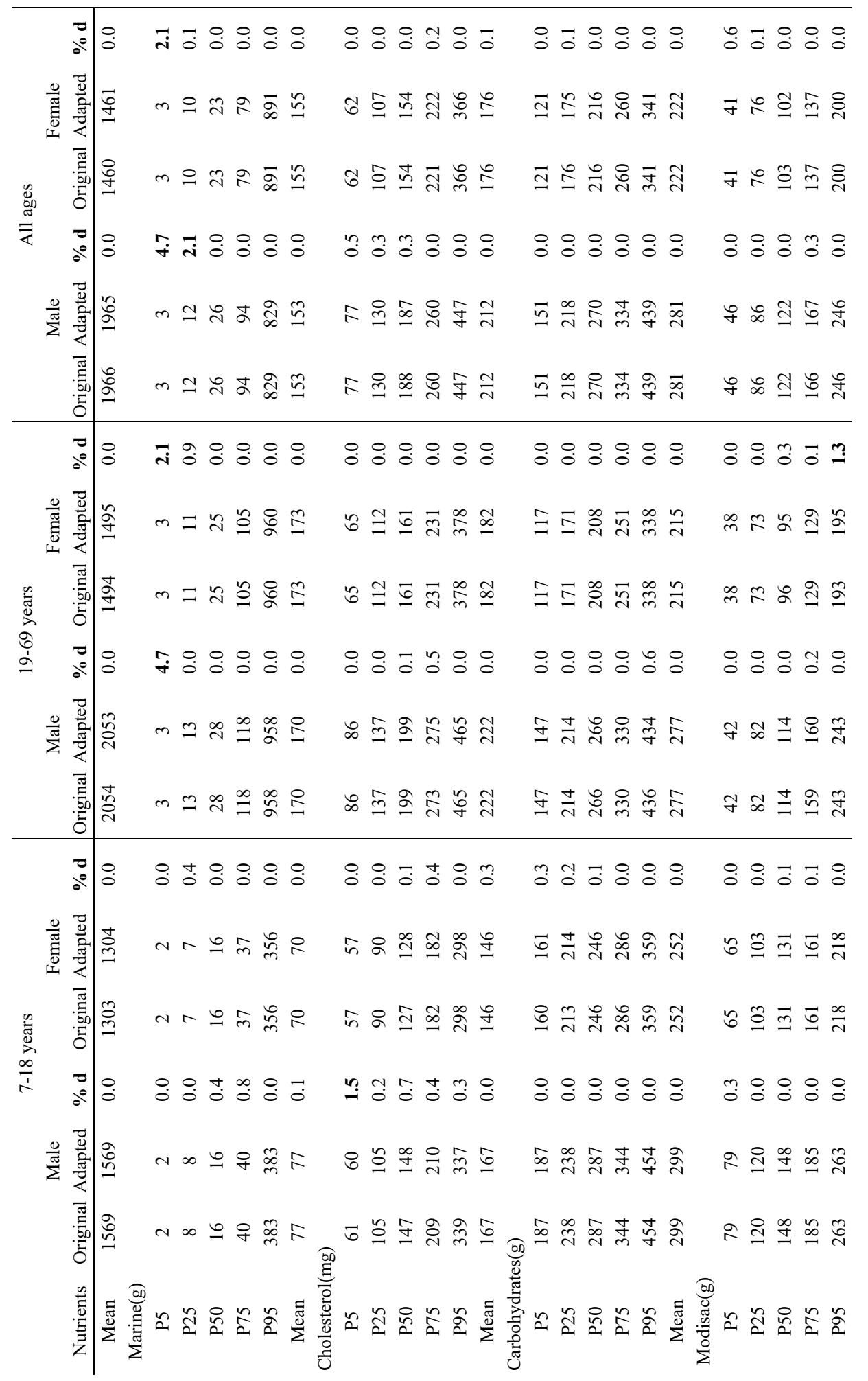




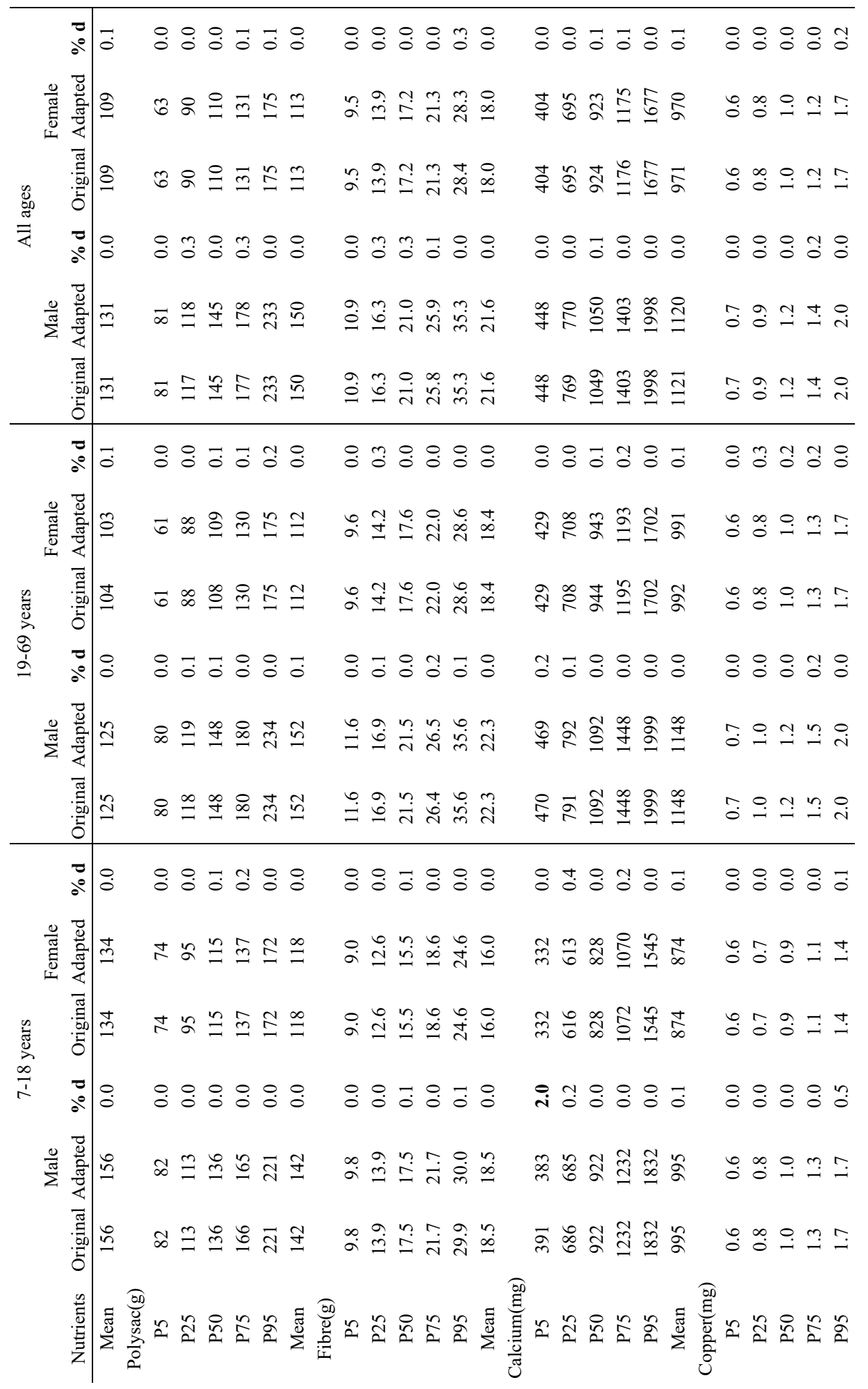




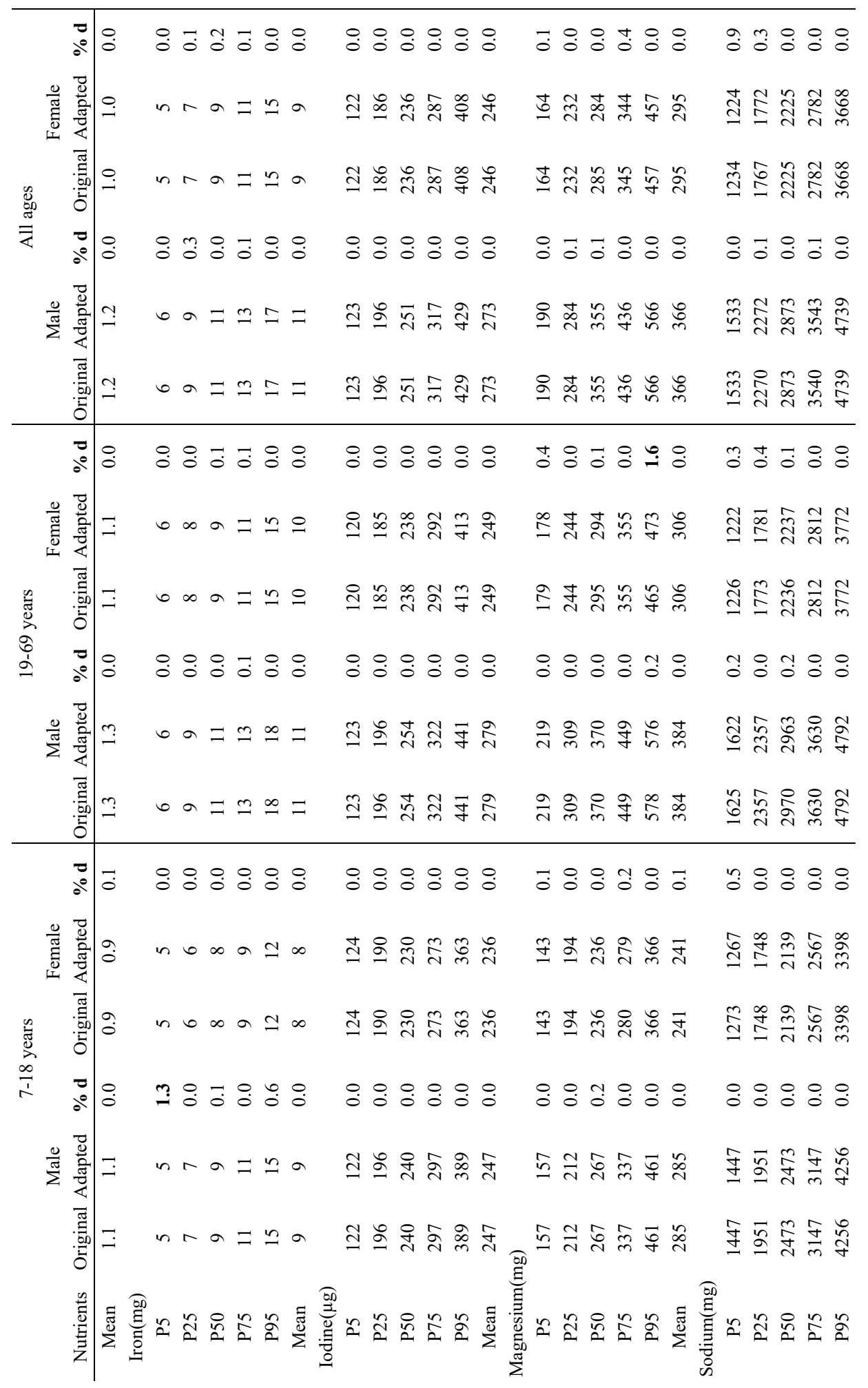




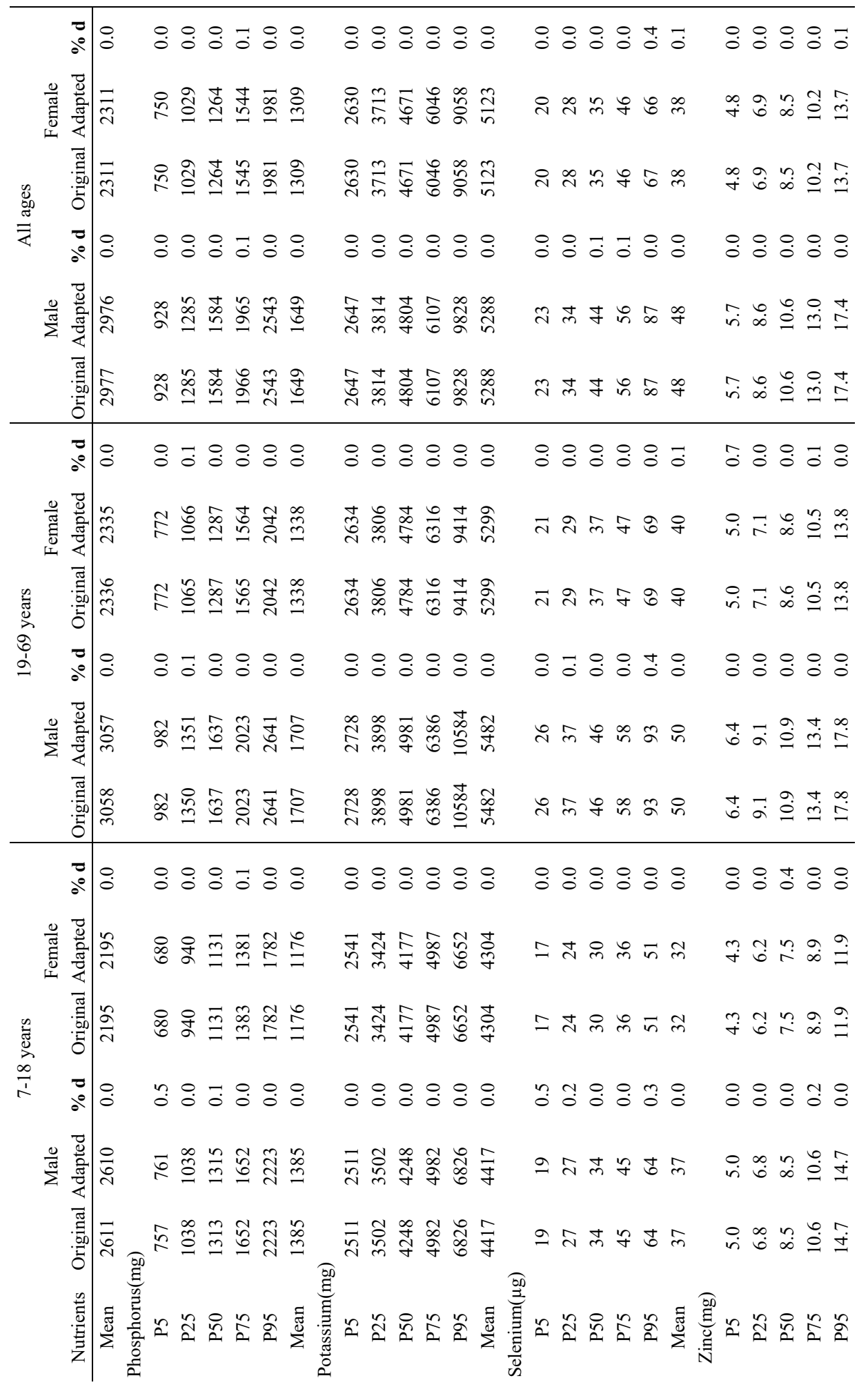




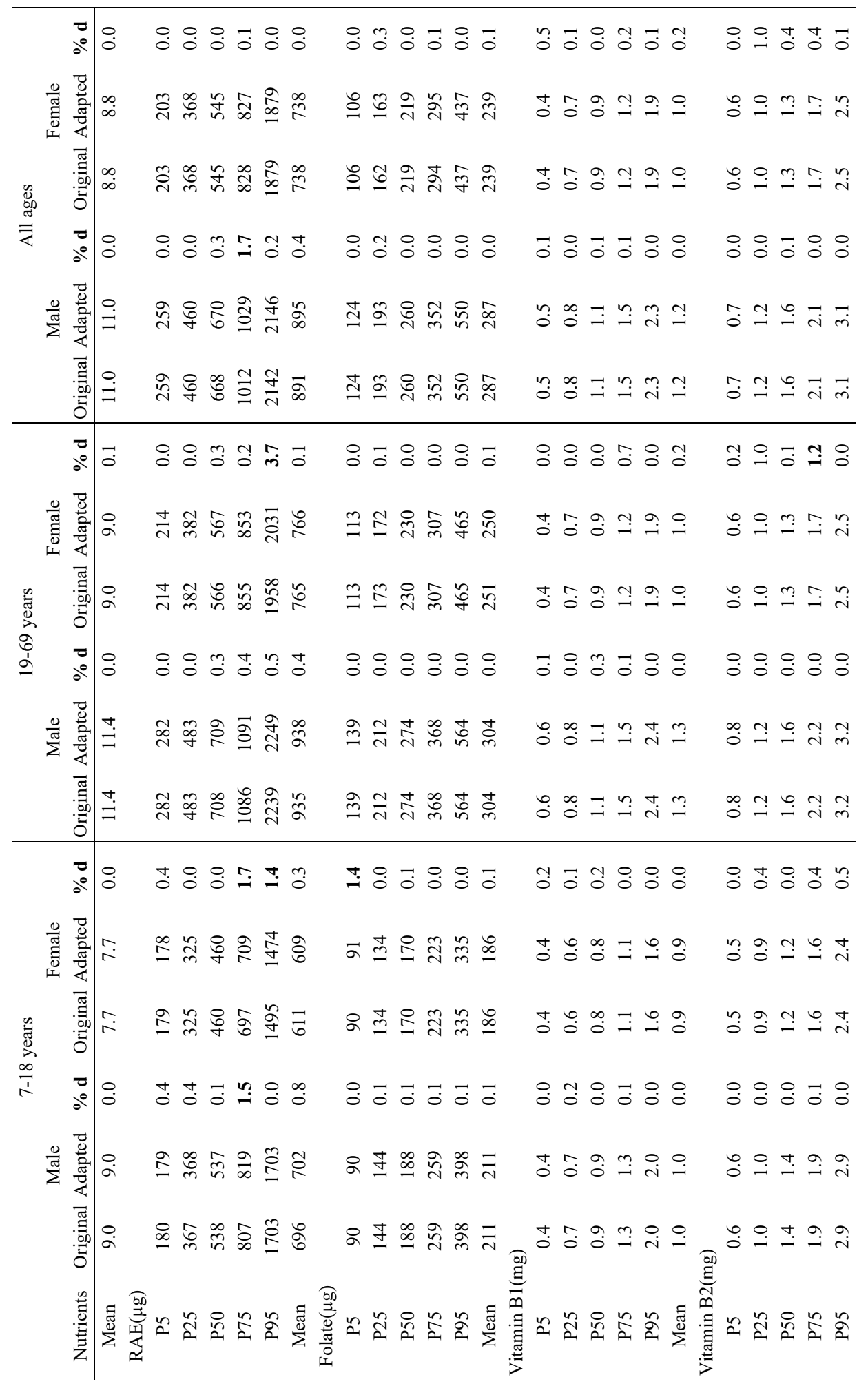




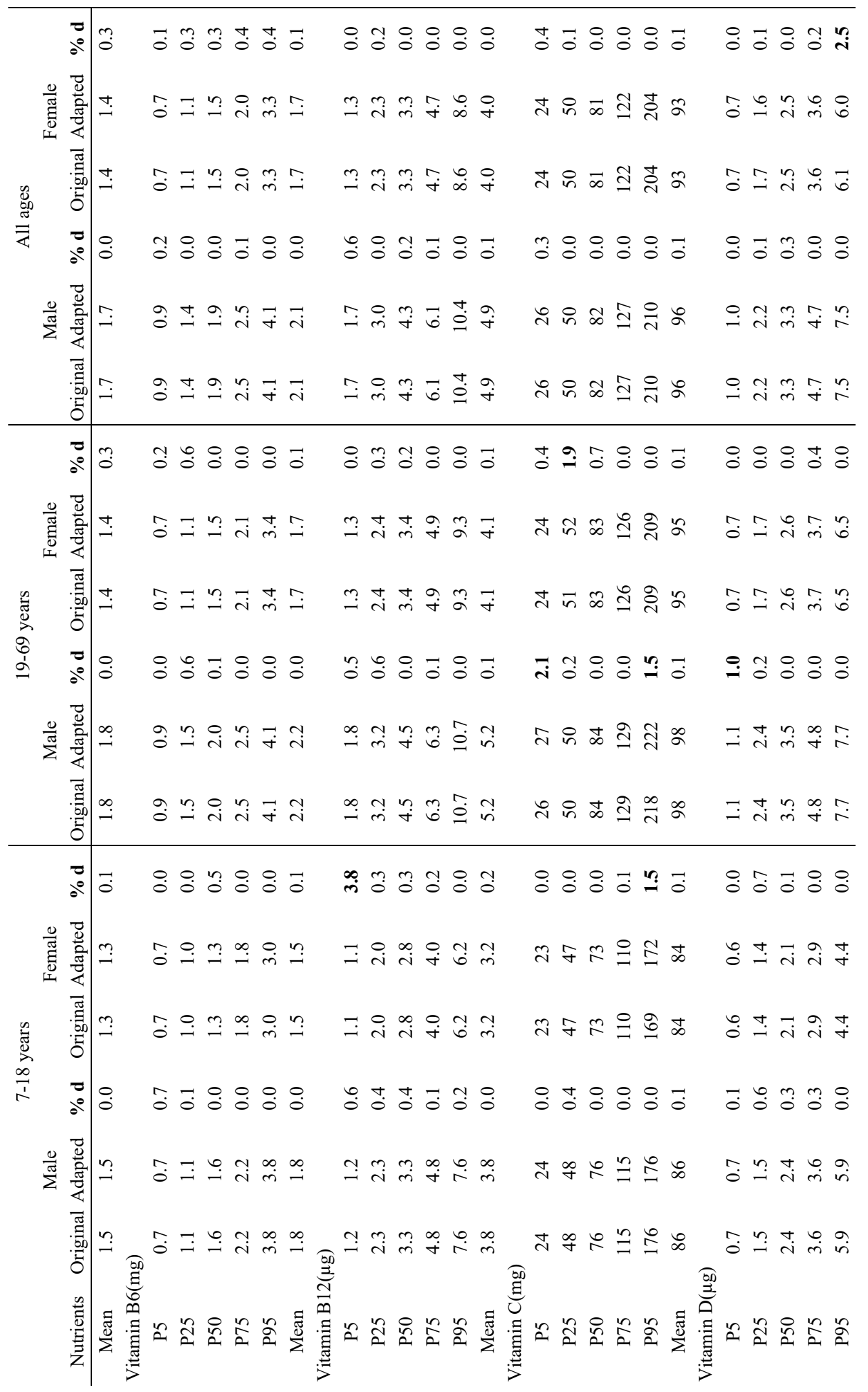


훙

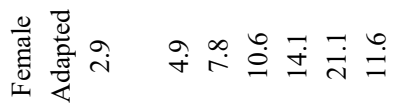

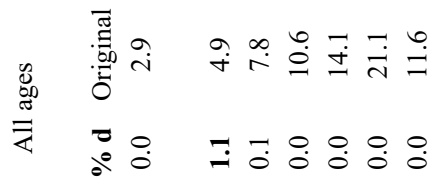

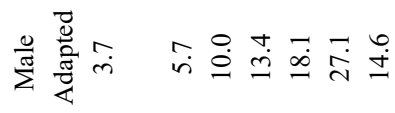

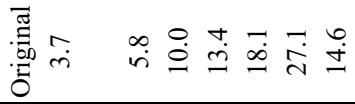

뭉

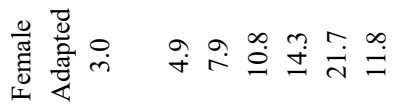

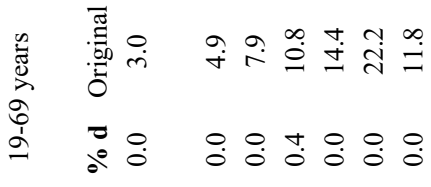

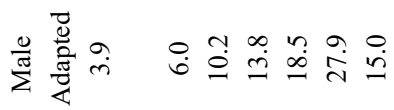
氶 후

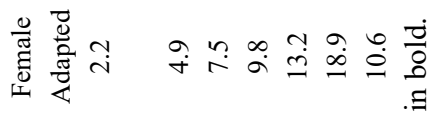

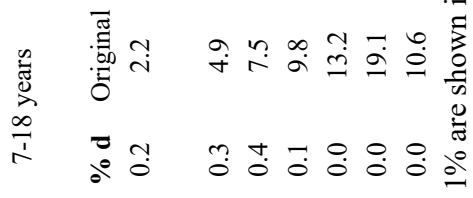

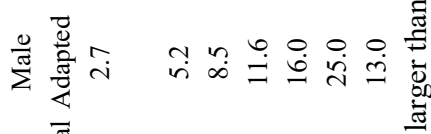

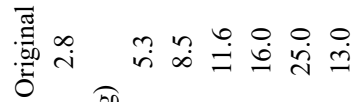

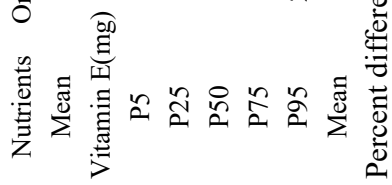




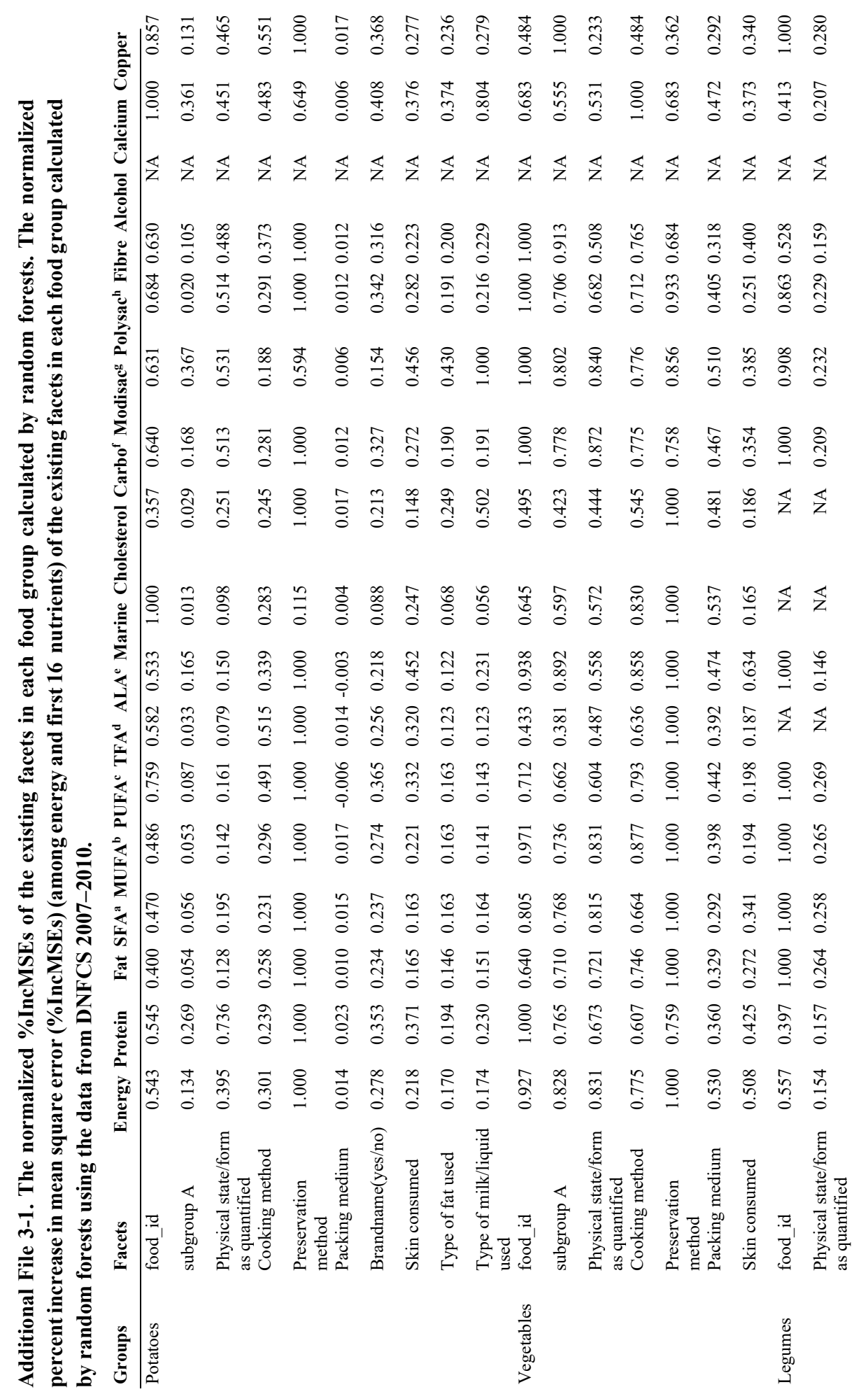




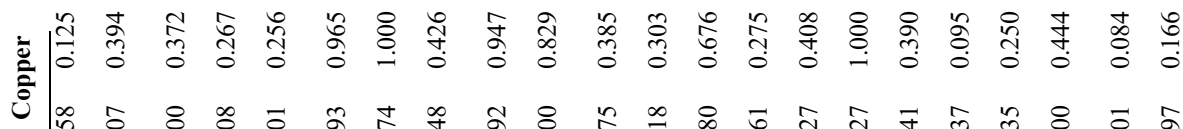

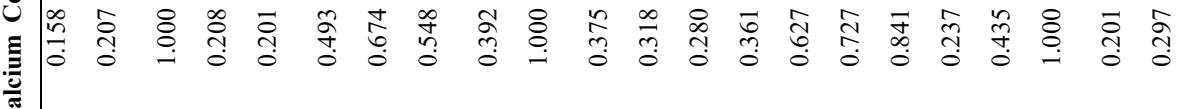

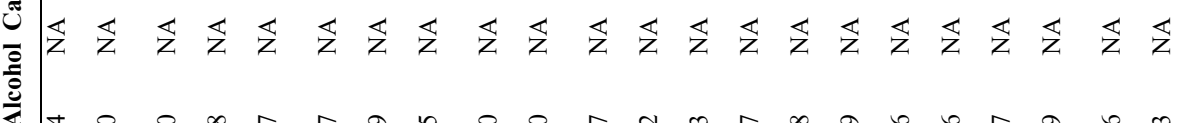

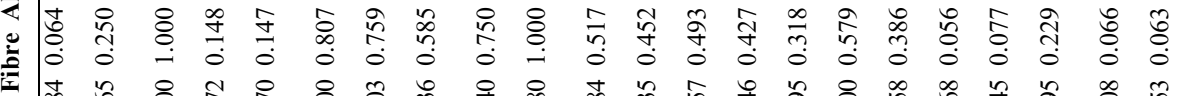

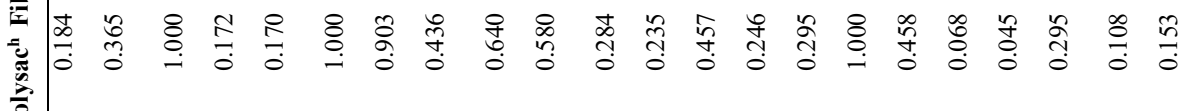

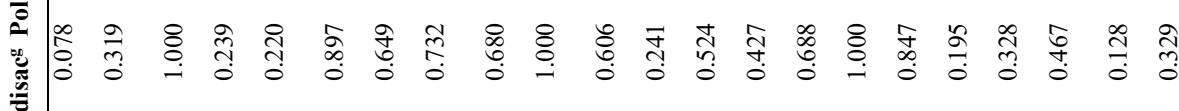

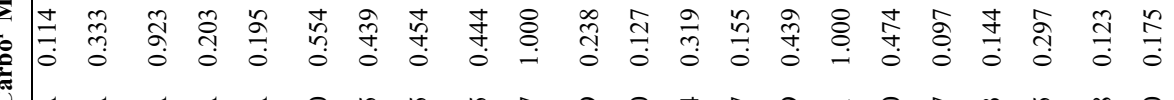

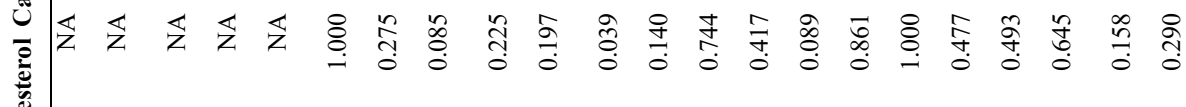

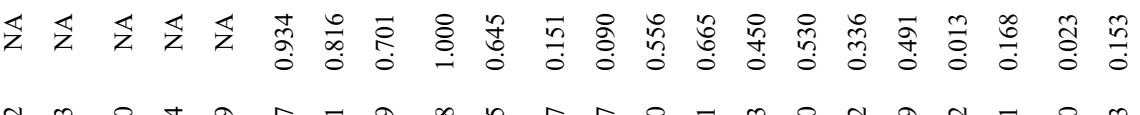

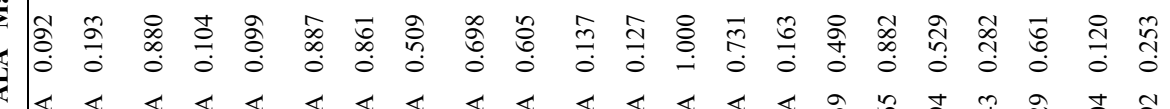

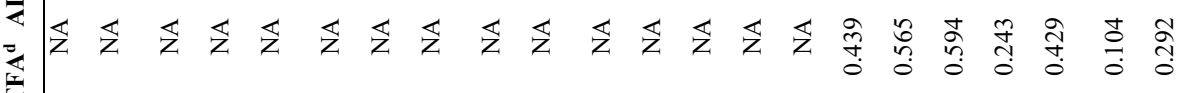

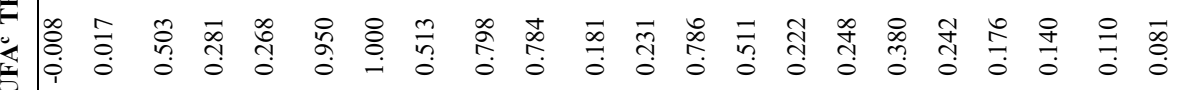

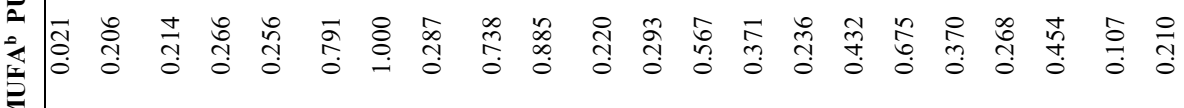

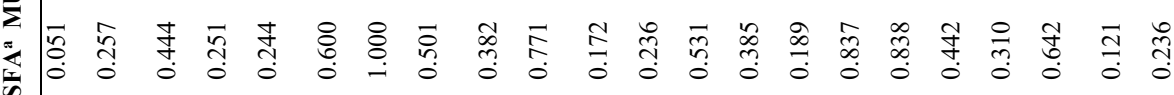

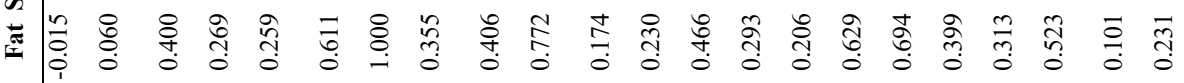

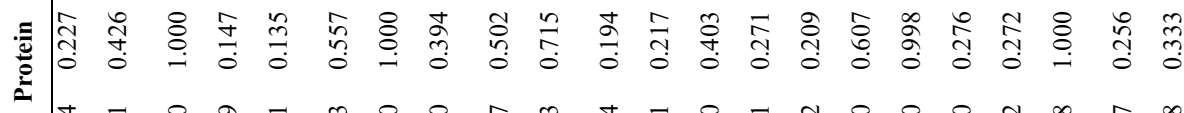

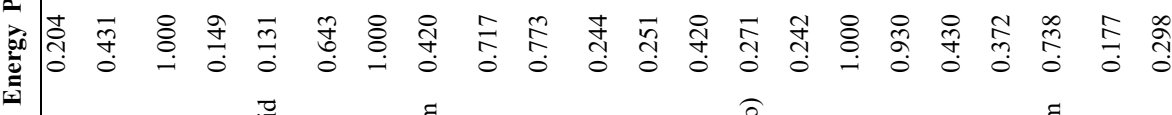

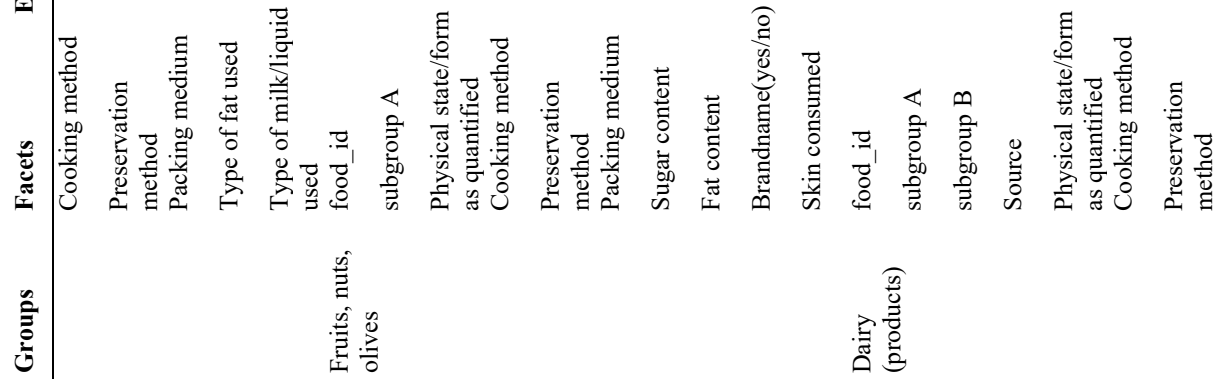




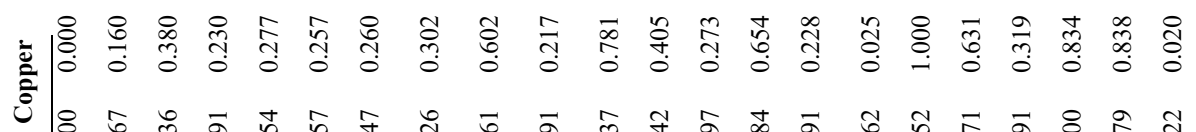

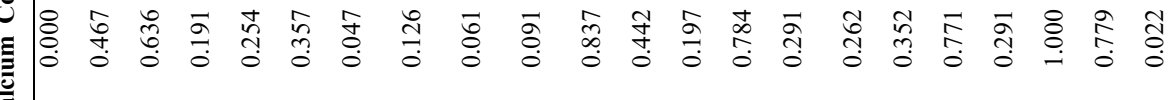

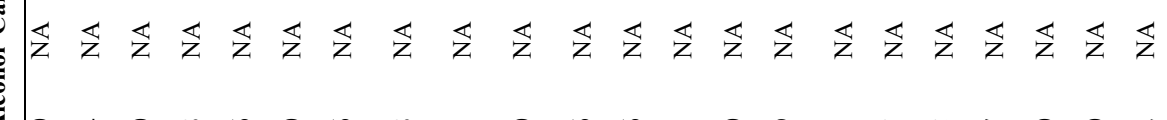

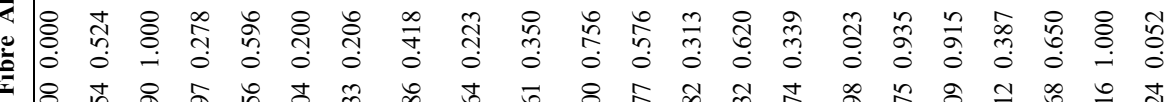
包

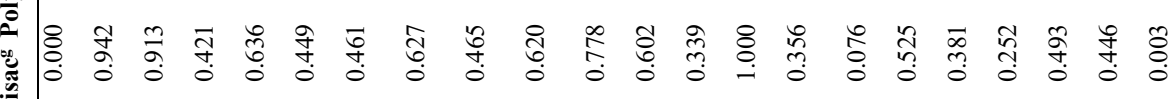

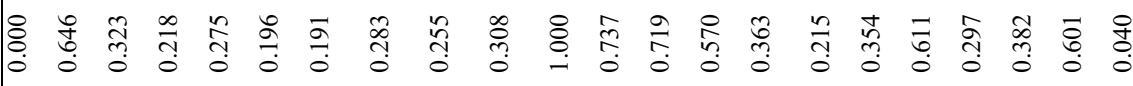

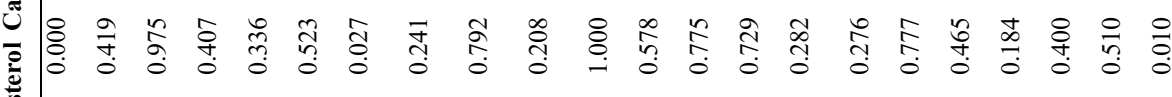

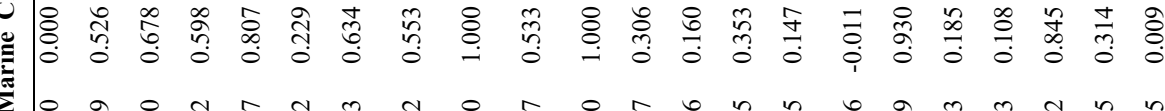

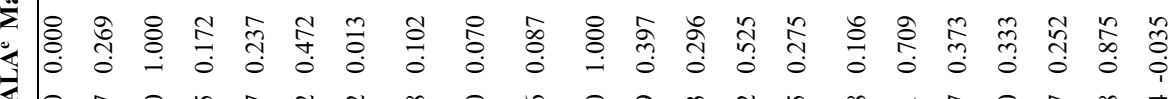

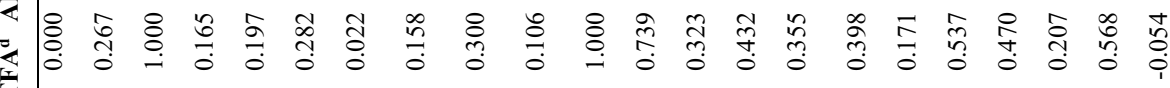
饪

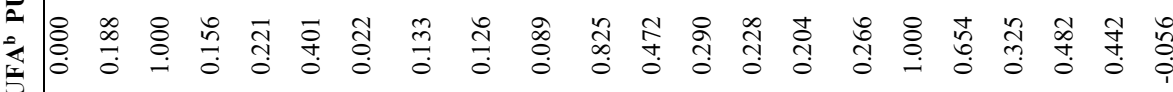

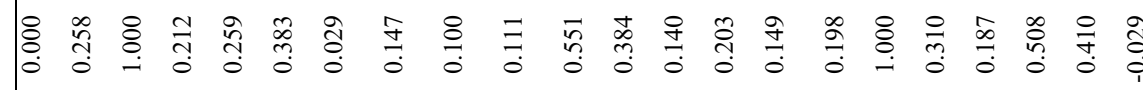

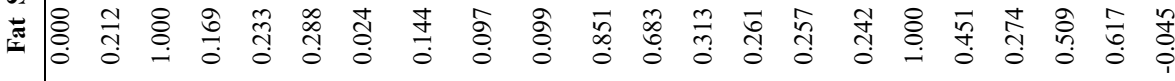
㐘

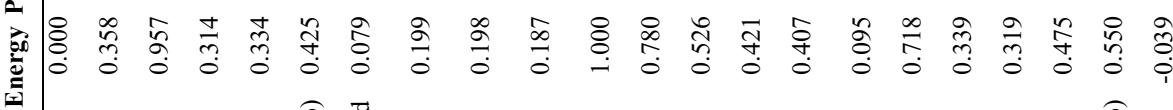

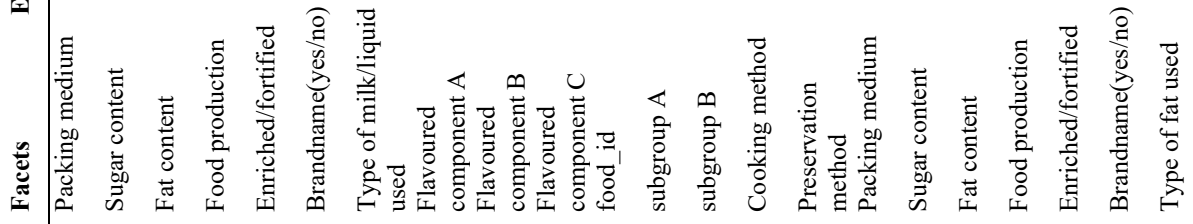
䓂 


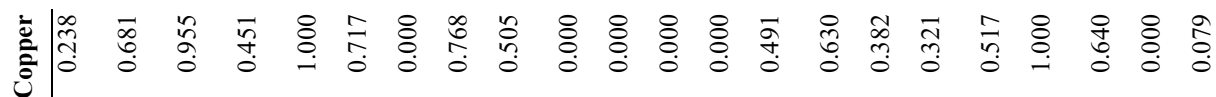

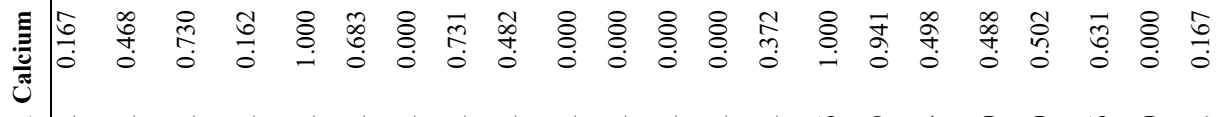

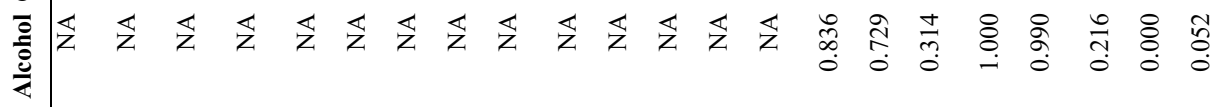

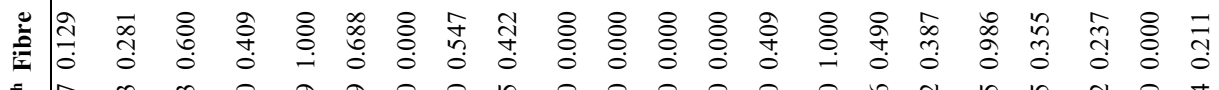

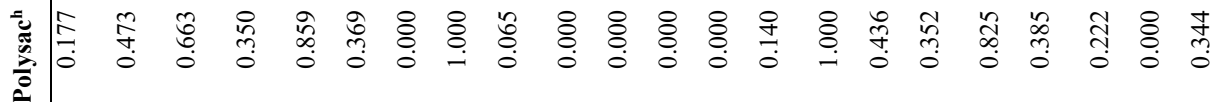

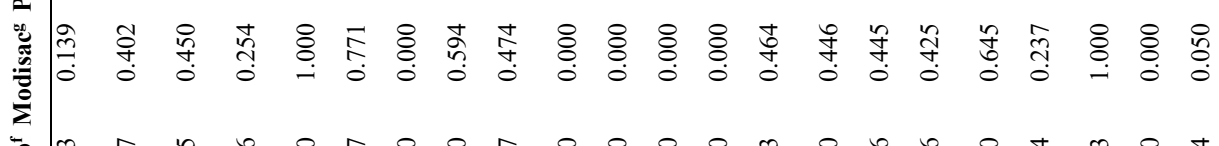

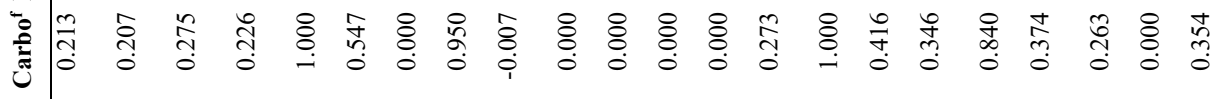

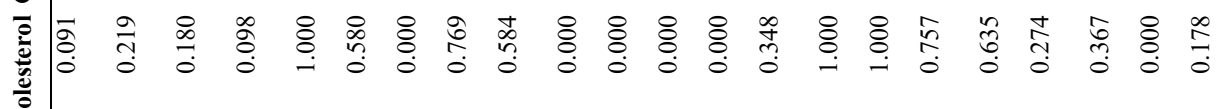

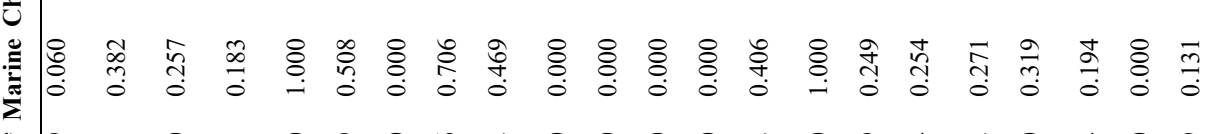

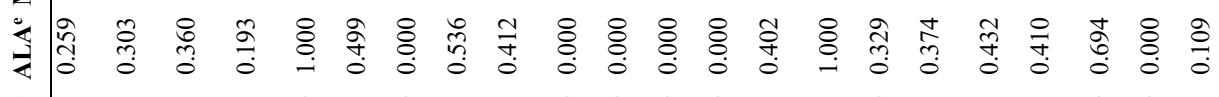

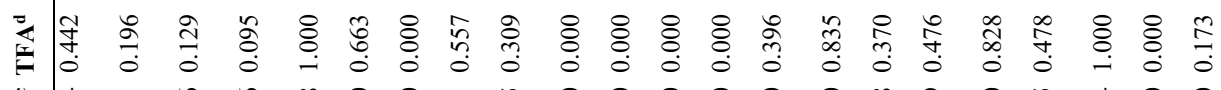

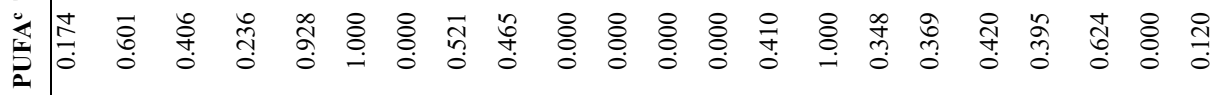

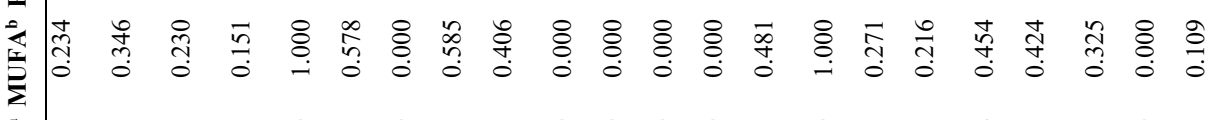
部得

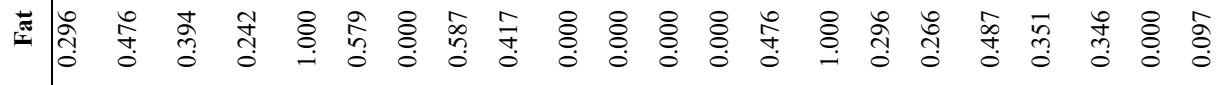

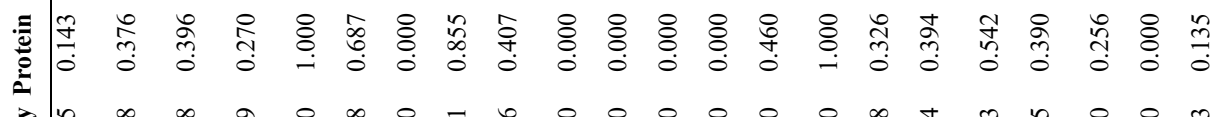

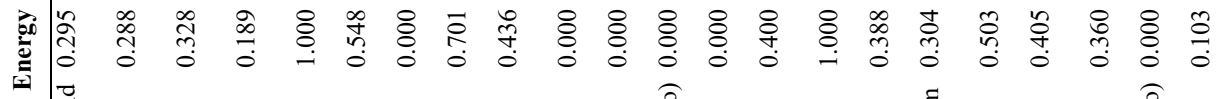

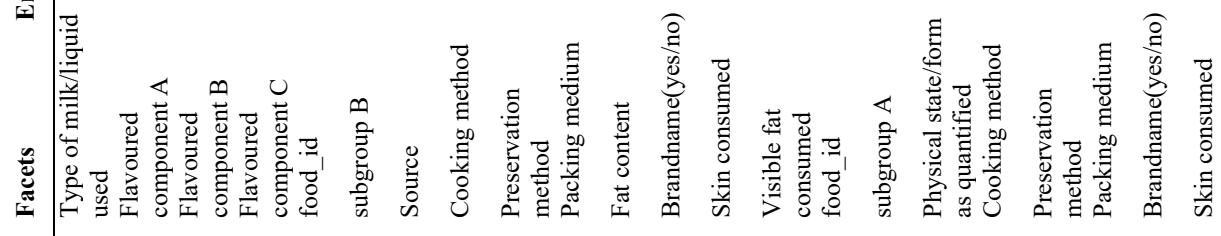

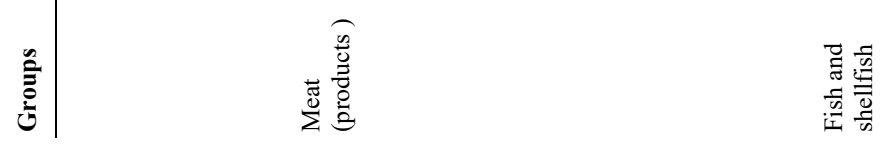




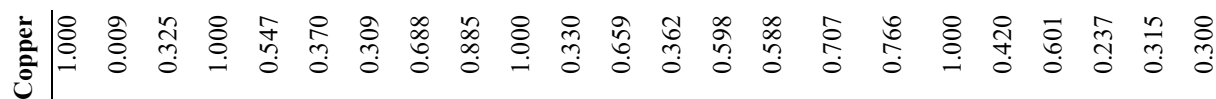

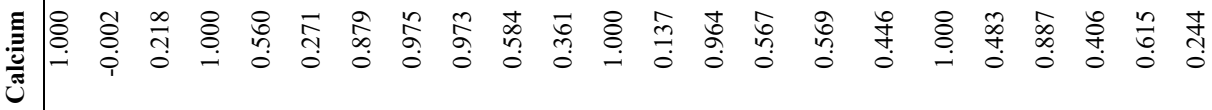

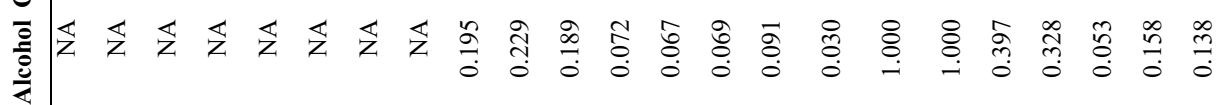

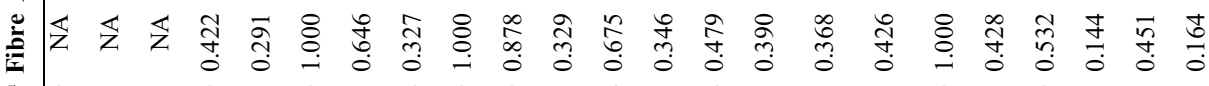

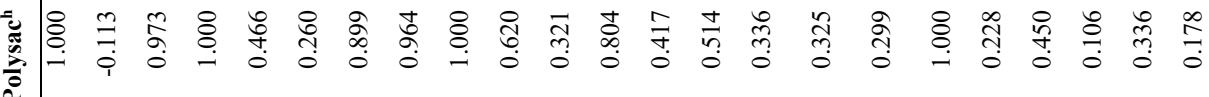

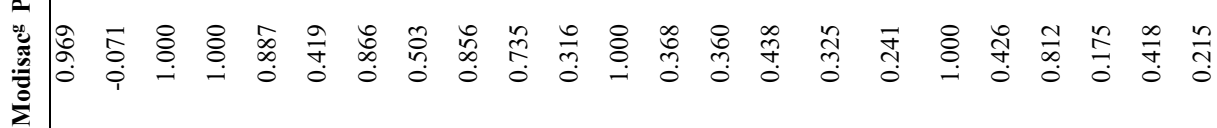

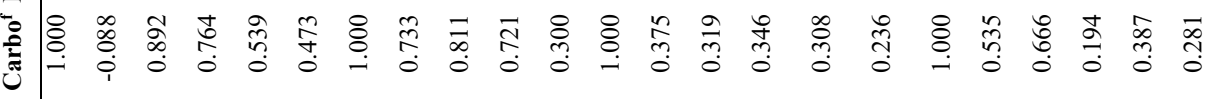

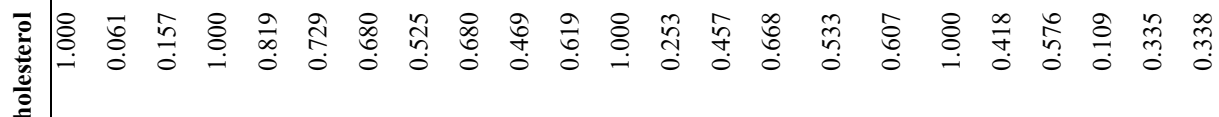

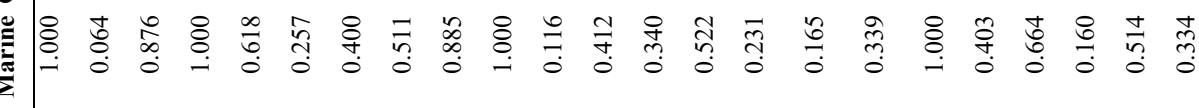

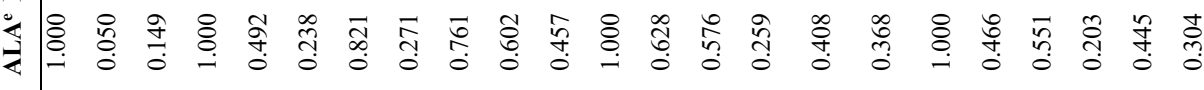

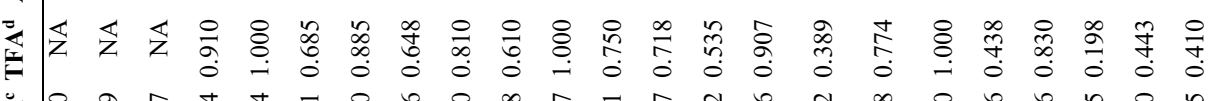

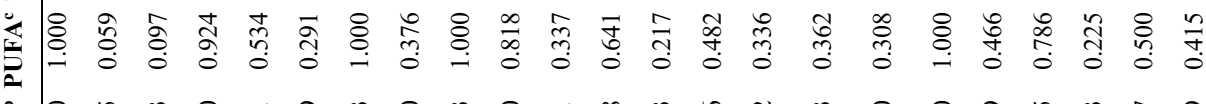

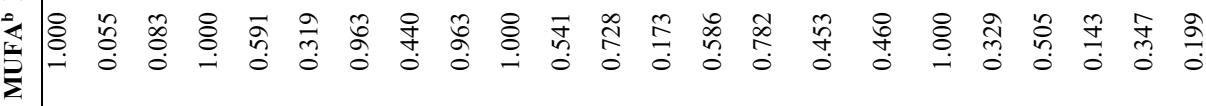

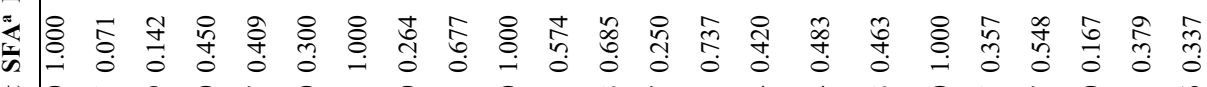

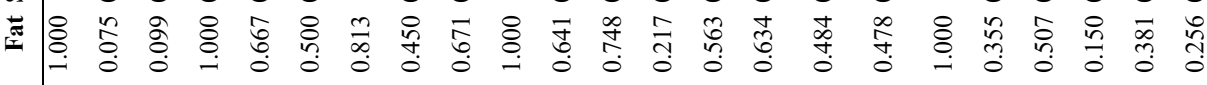

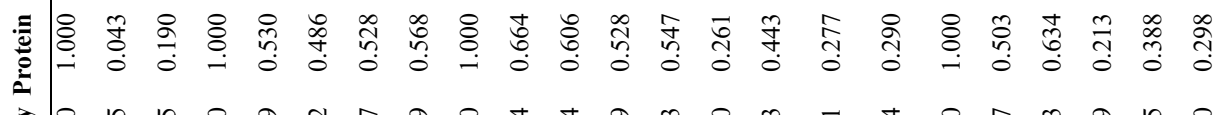

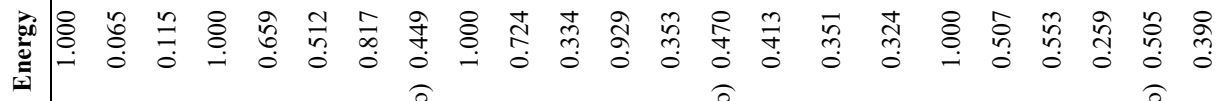

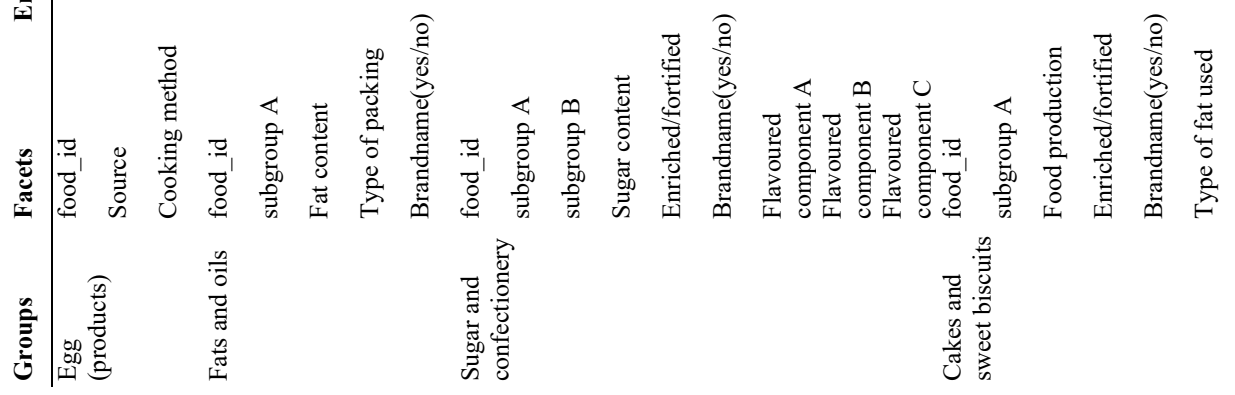




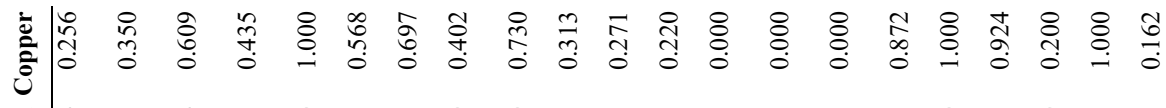

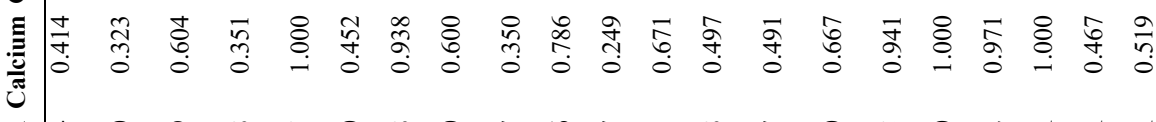

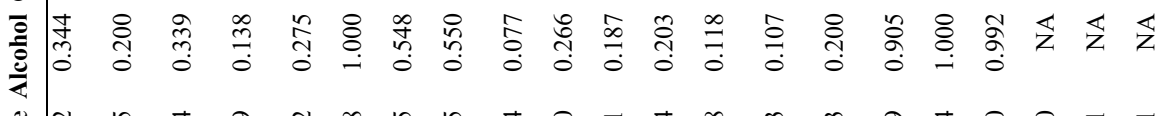

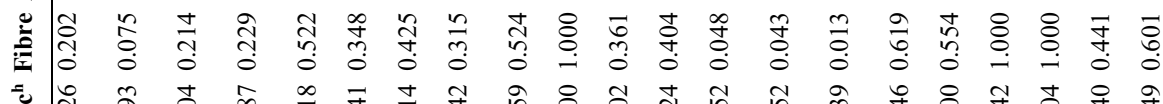

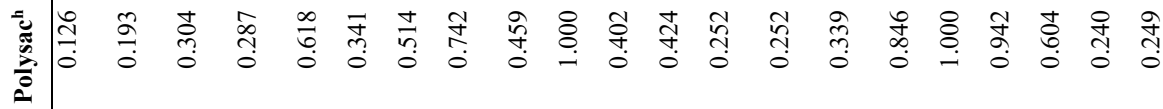

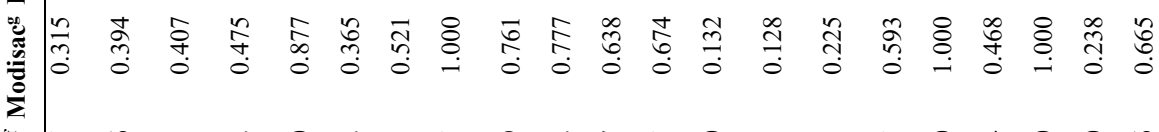

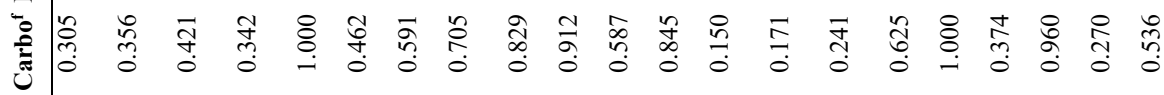

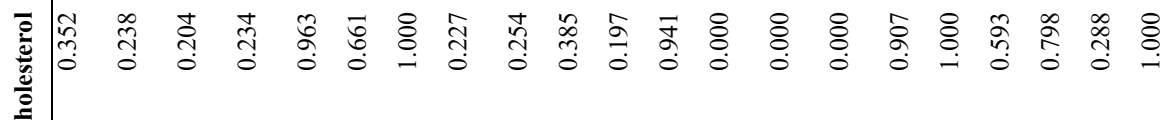

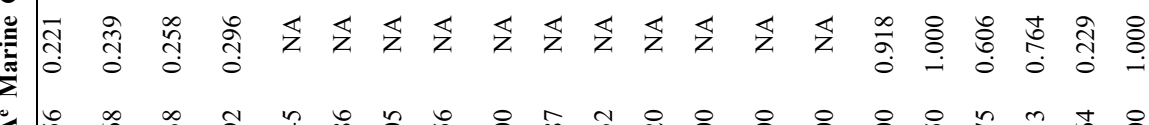

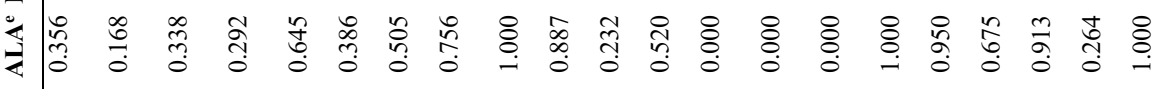

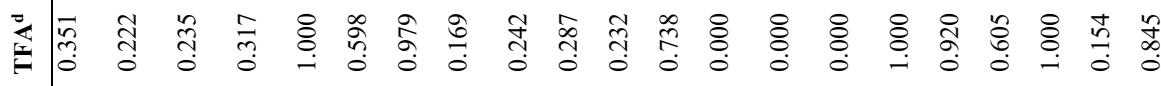

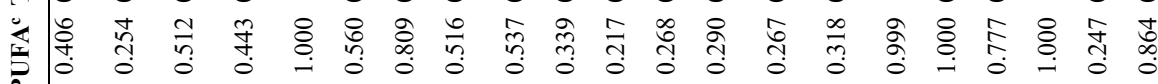

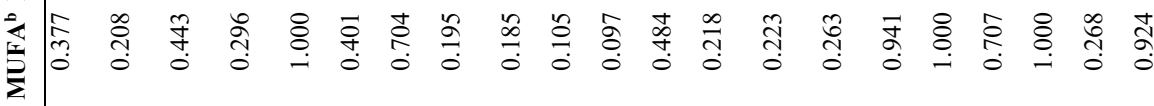

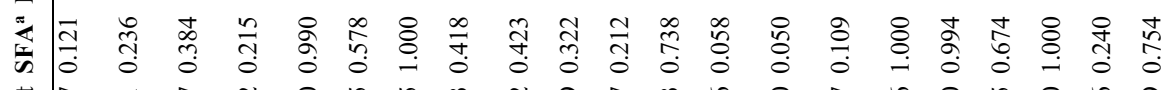

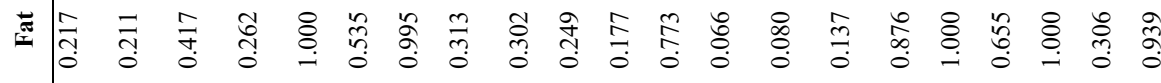

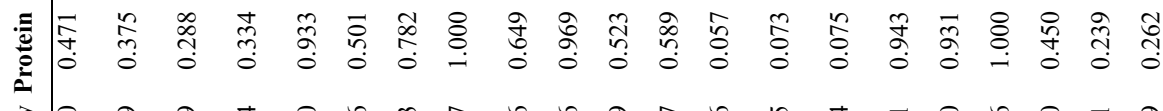

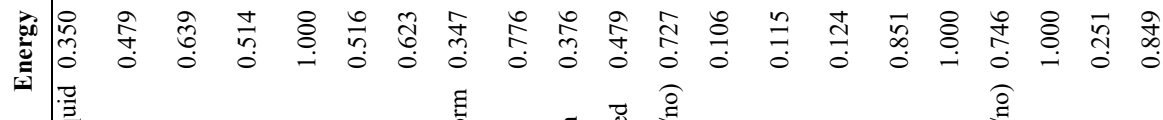

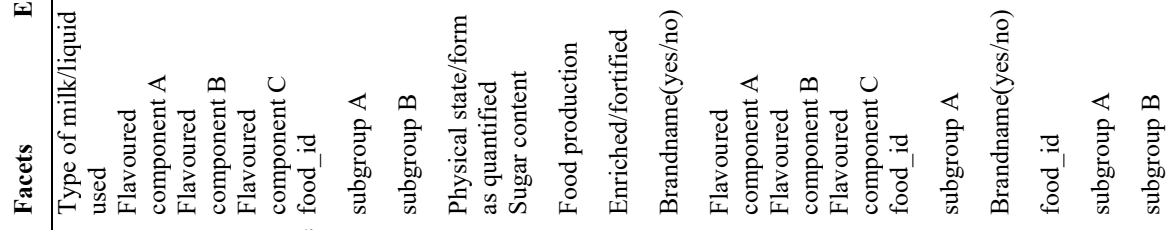

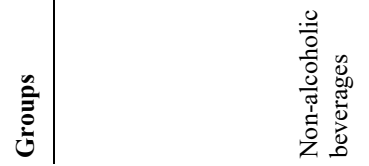




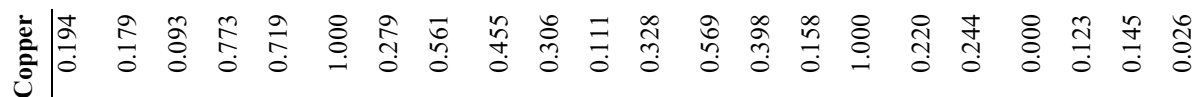

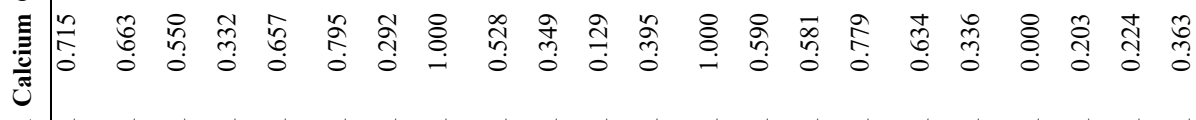

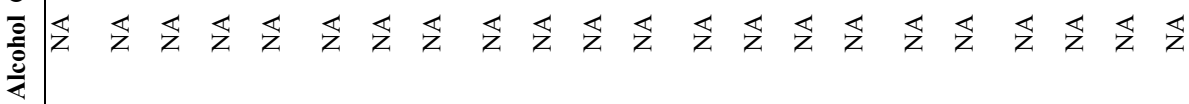

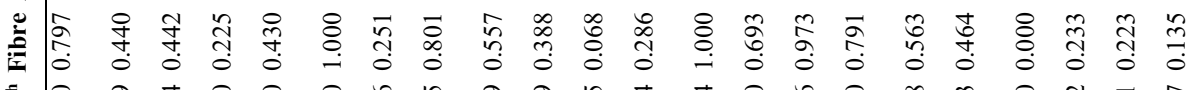

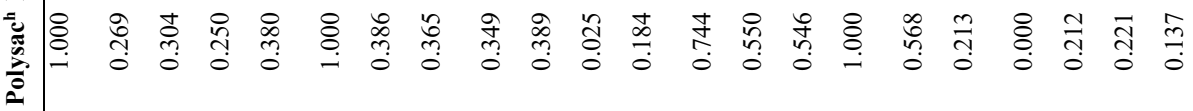

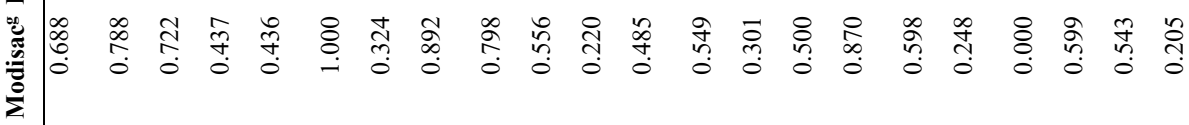

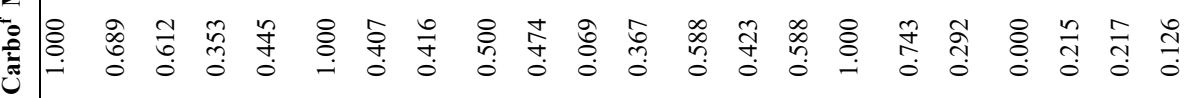

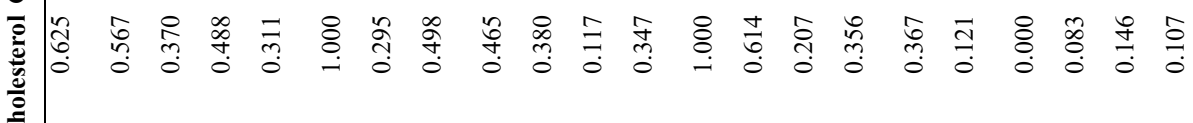

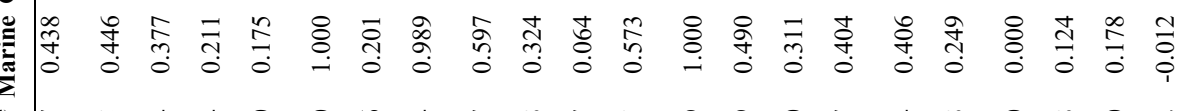

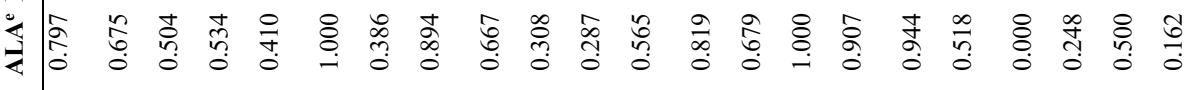

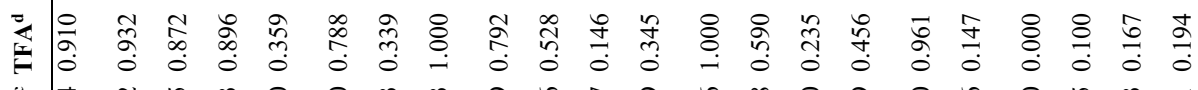

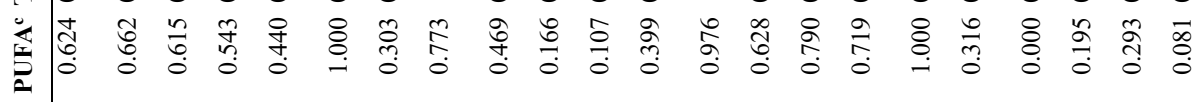

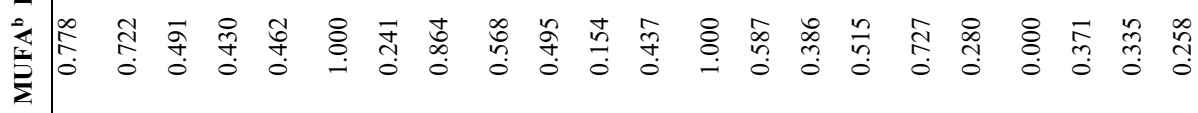

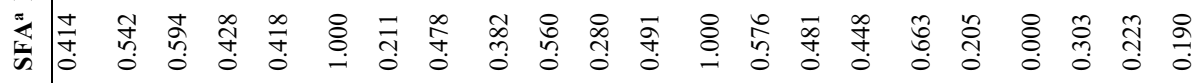

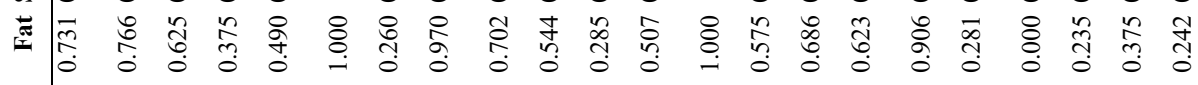

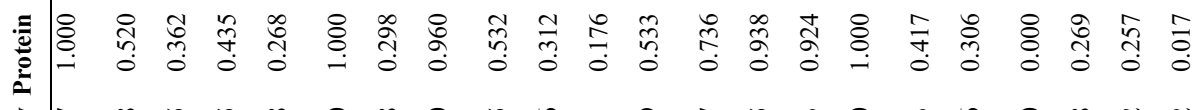

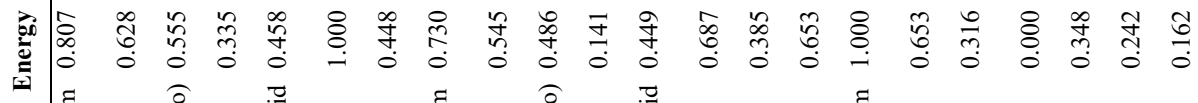

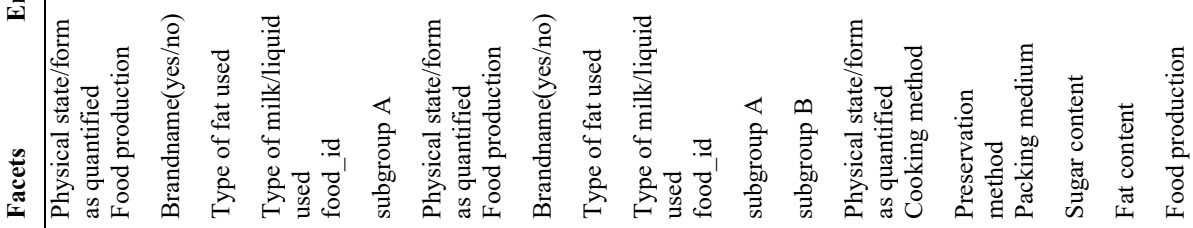

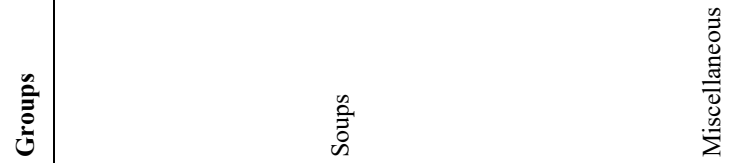




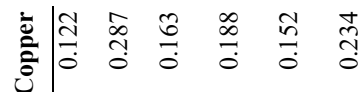

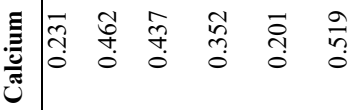

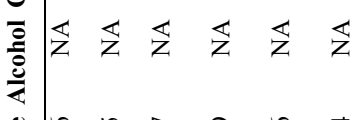
章

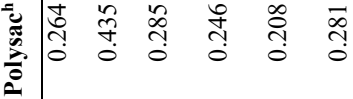
:

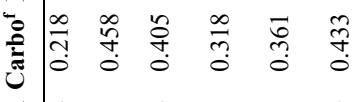
竎 巳一 壳

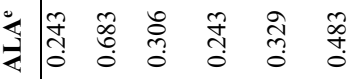
选 造

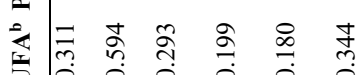
$\sum \mid \begin{array}{llllll}0 & 0 & 0 & 0 & 0 & 0\end{array}$

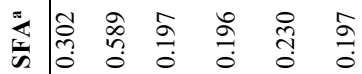

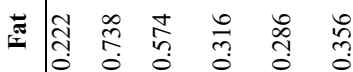
:

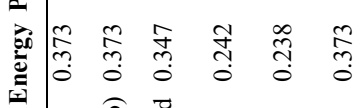

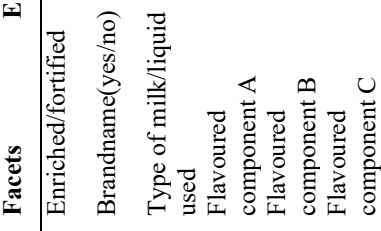
产 


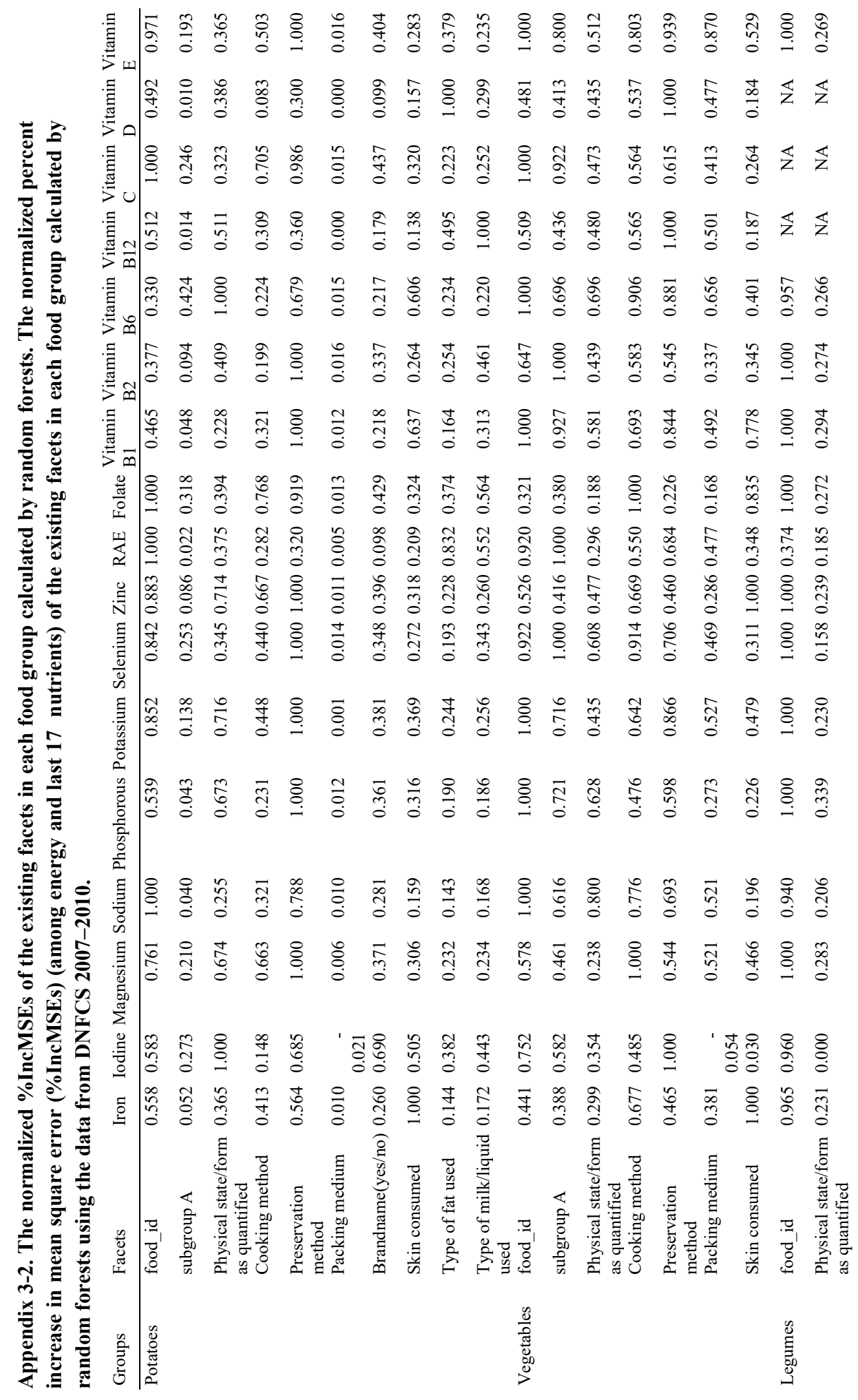




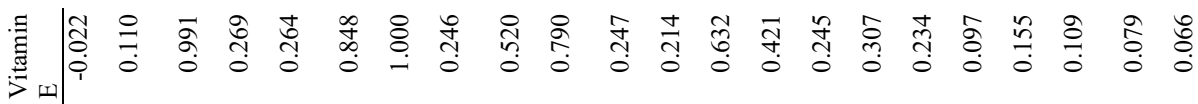

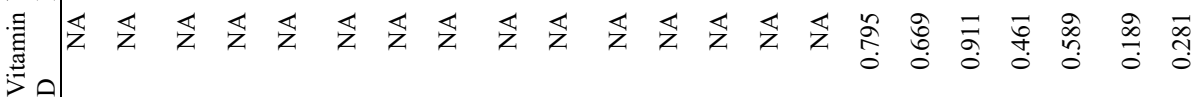

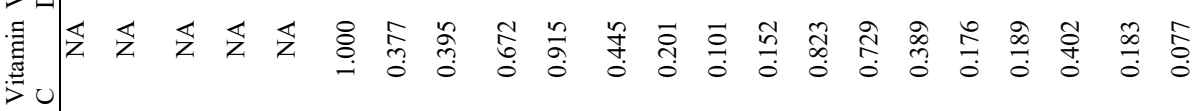
焉 表

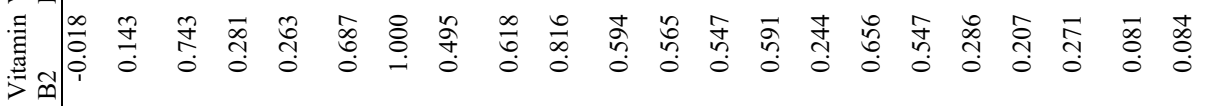
表

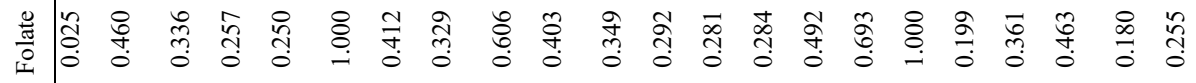

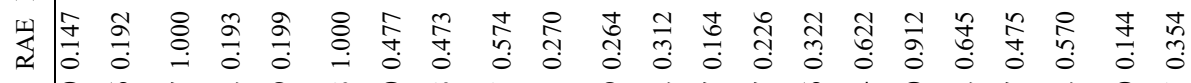

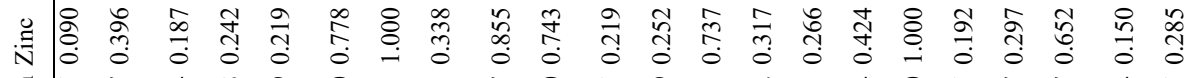

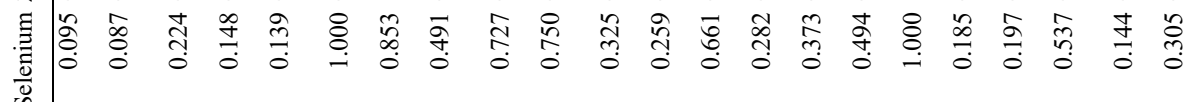

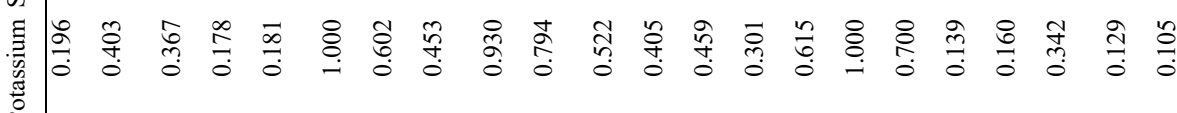

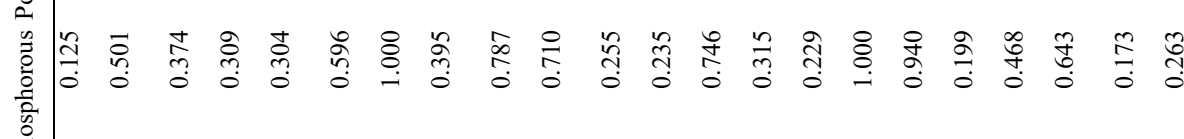

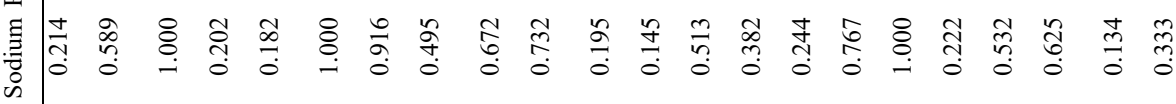

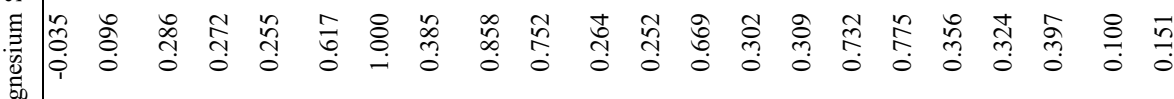
邹

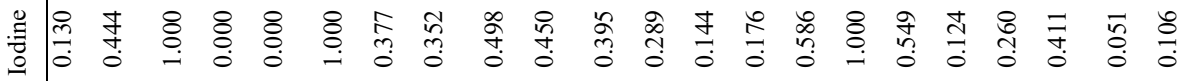

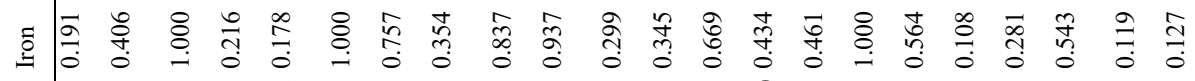

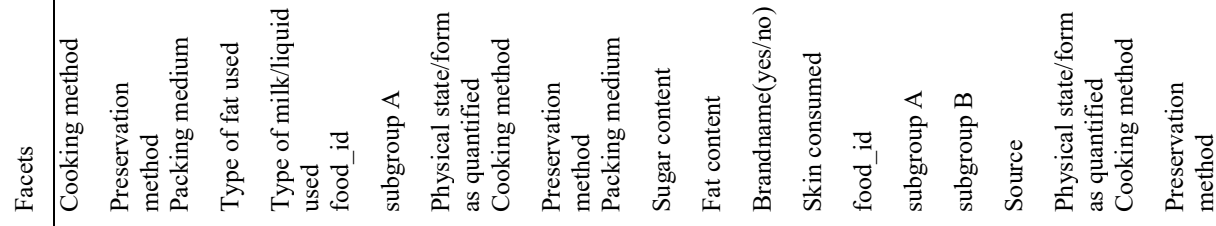

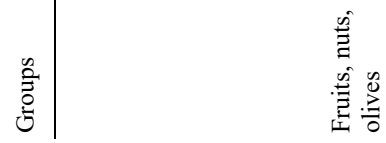




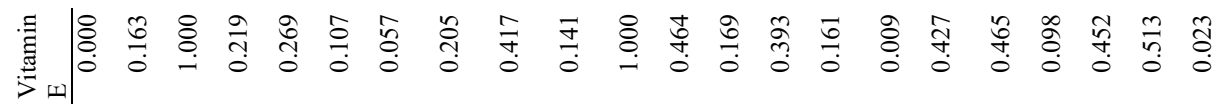
言

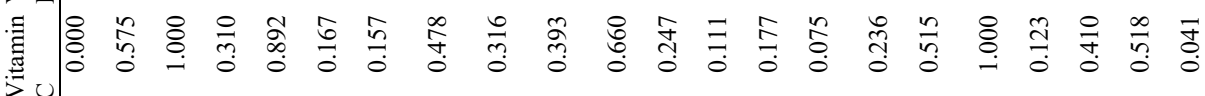
表 寻

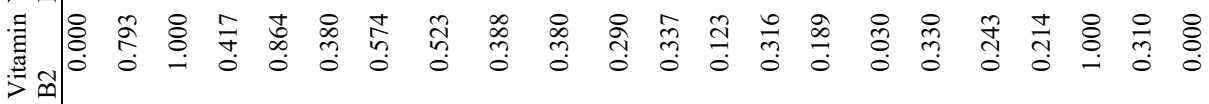

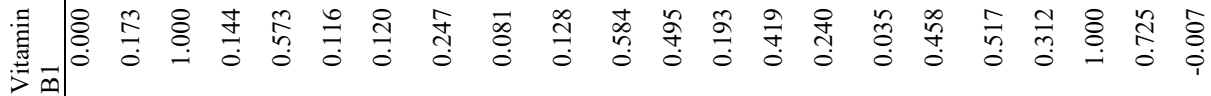

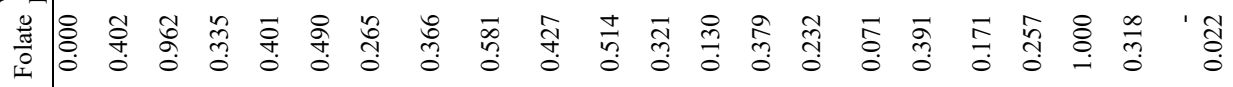
湈

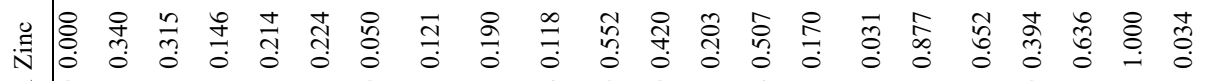

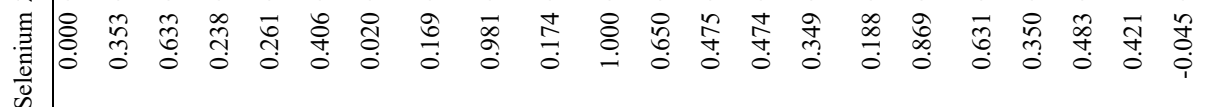
势

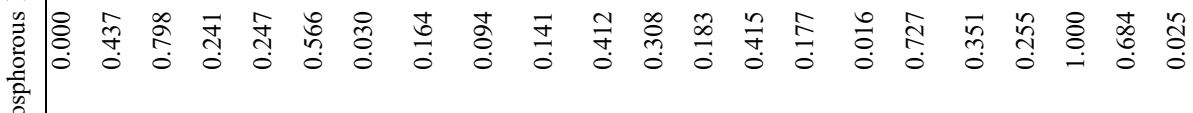

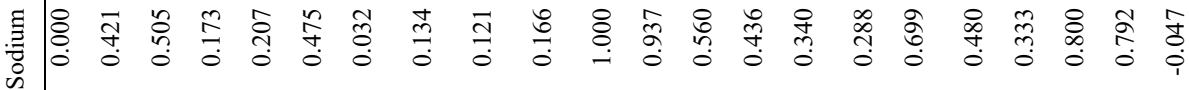

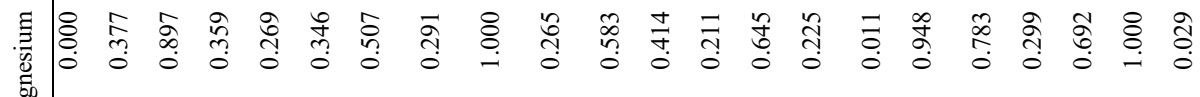
党

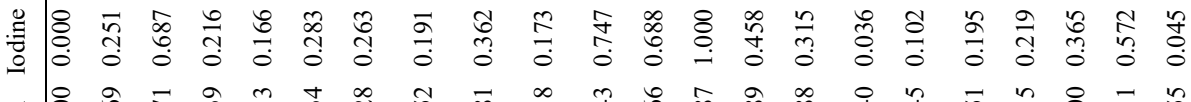

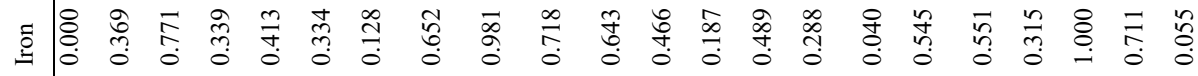

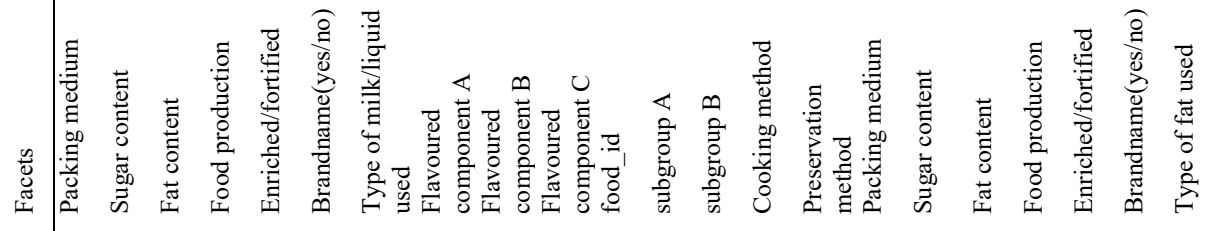




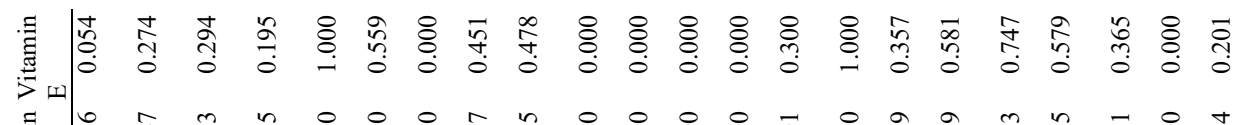
表

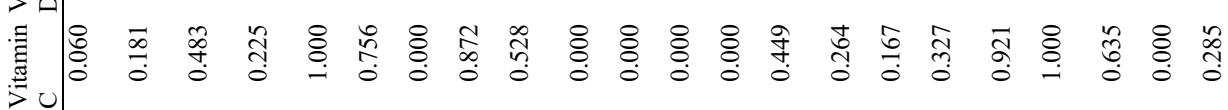

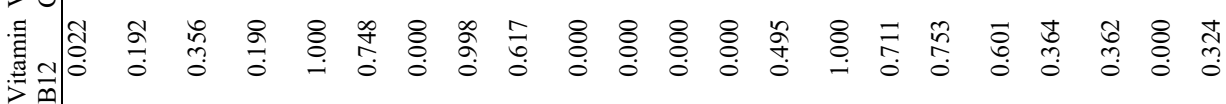

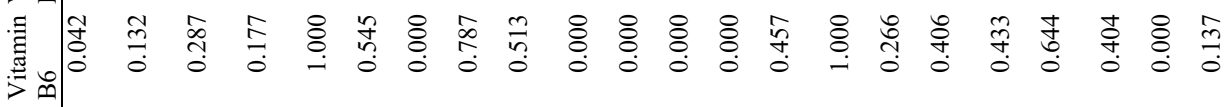
言

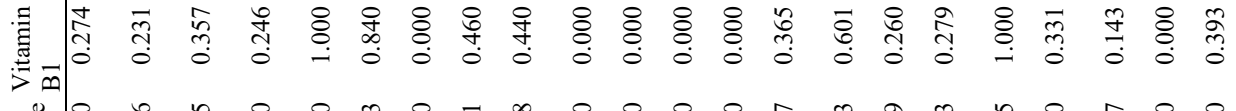

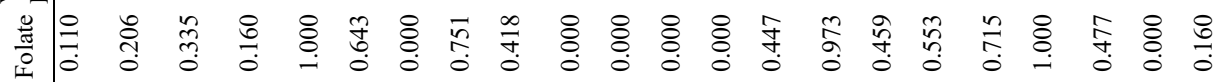

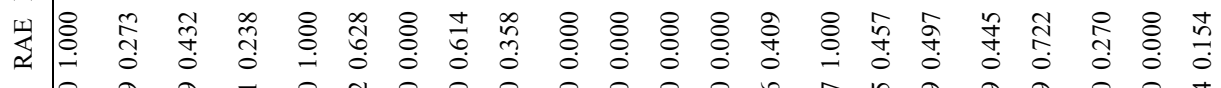

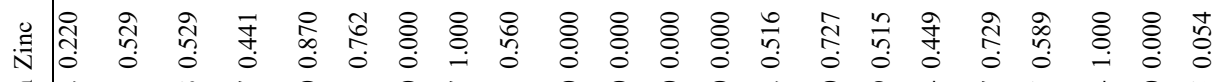

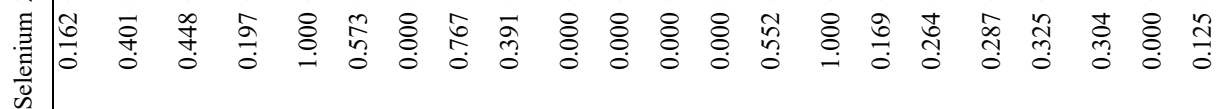

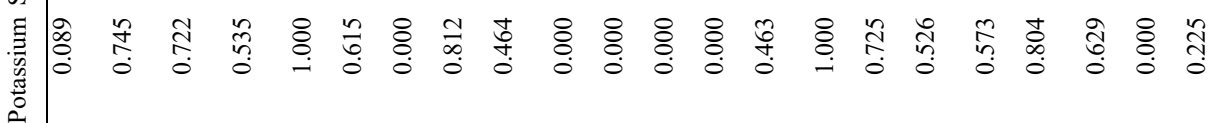

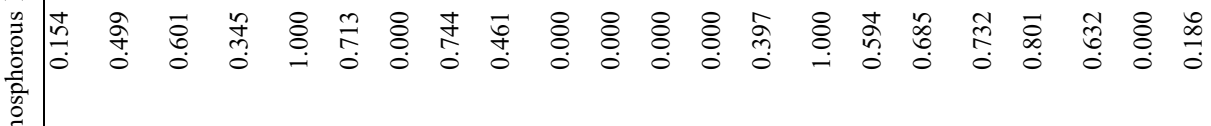

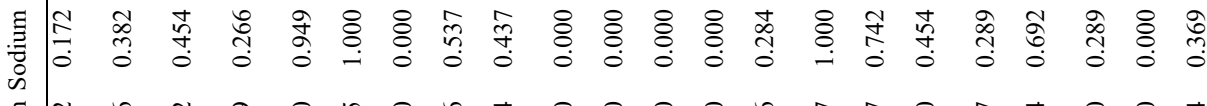

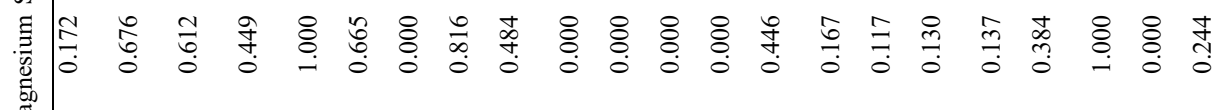
曾

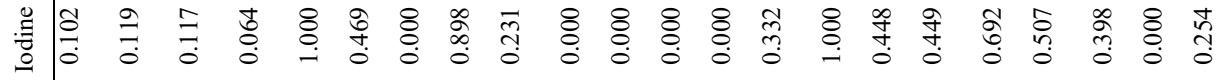

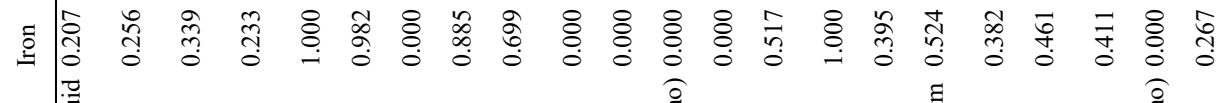

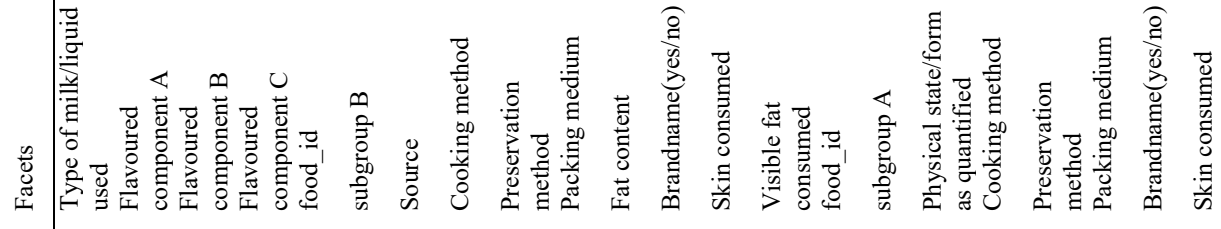

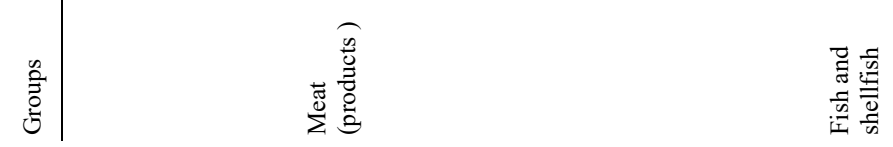




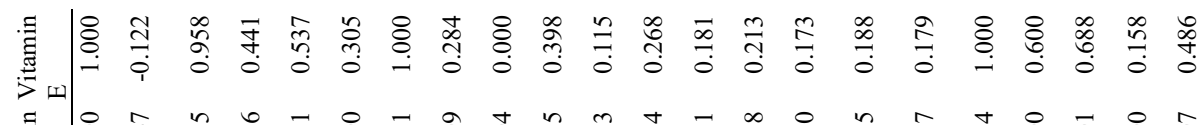

泉

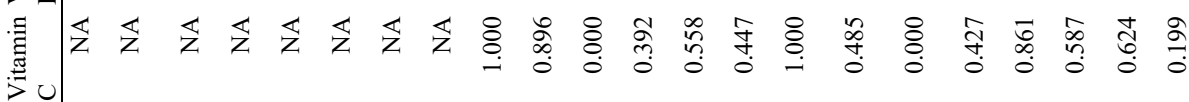
焉 丞 言

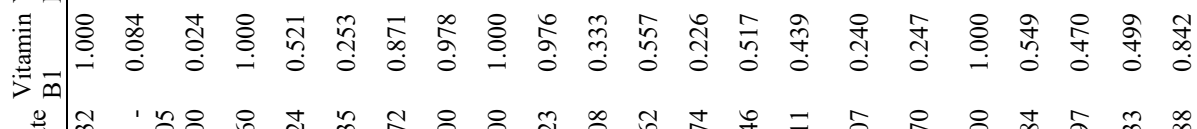

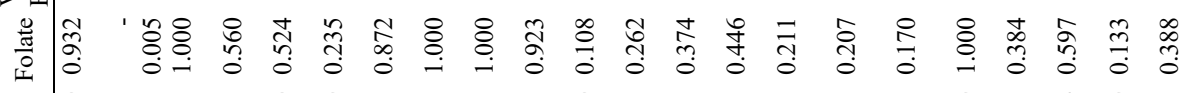

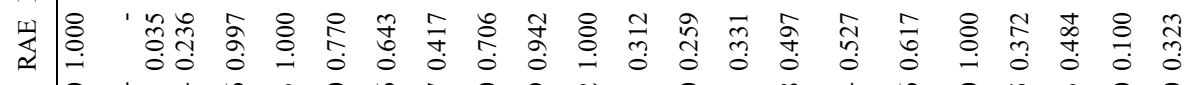

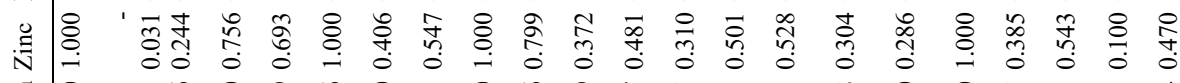

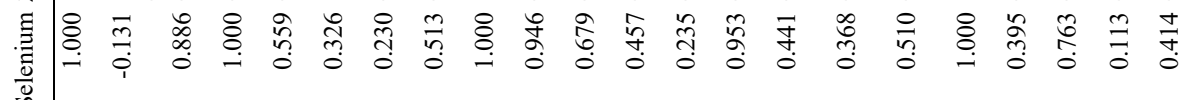

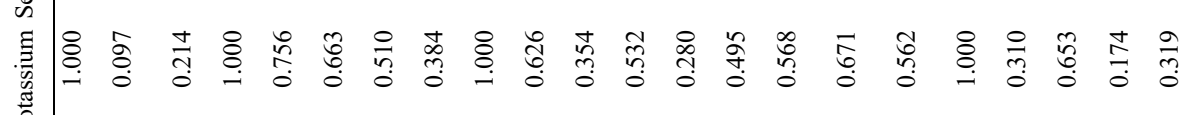
离

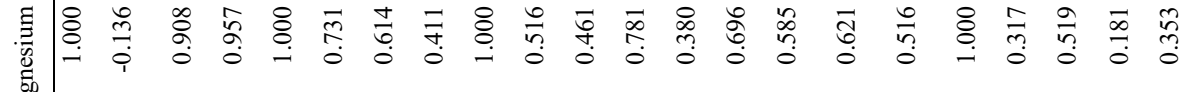

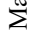

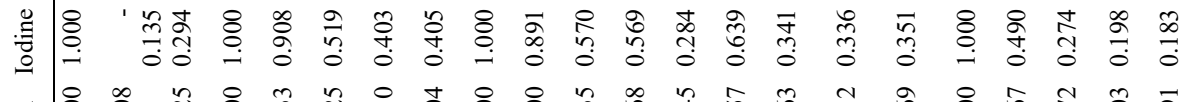

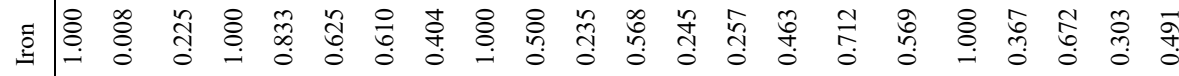

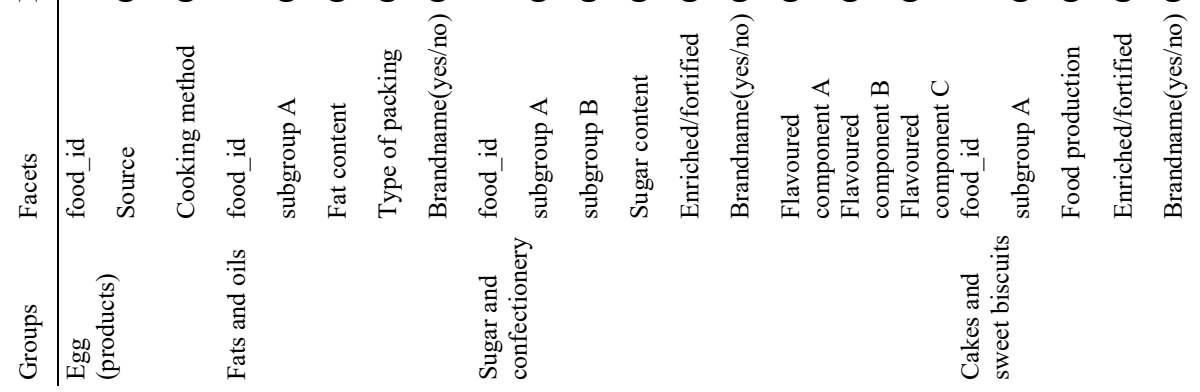




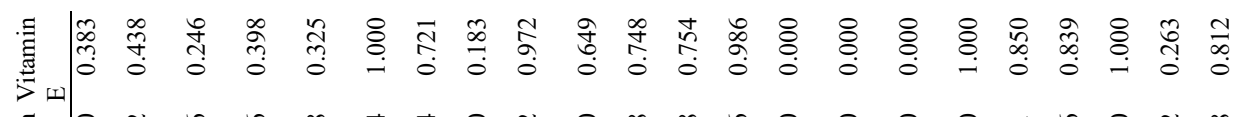
聲 衰

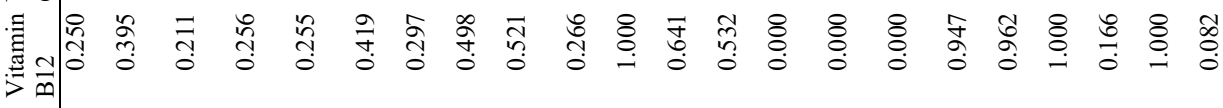

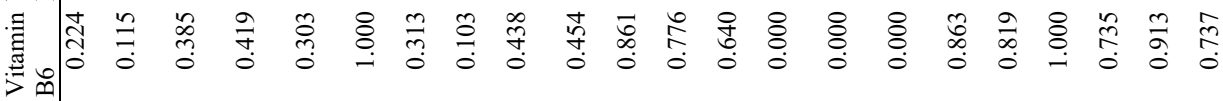

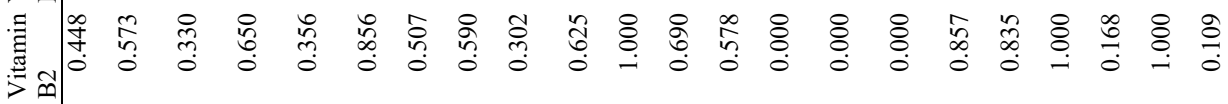

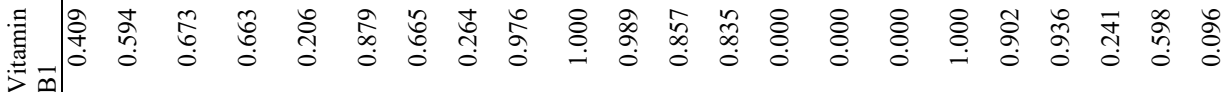

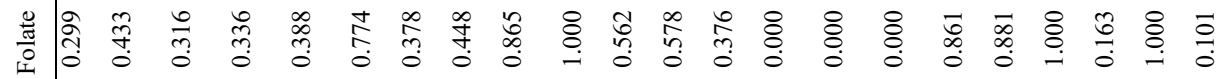

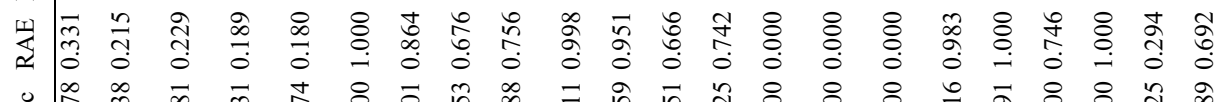

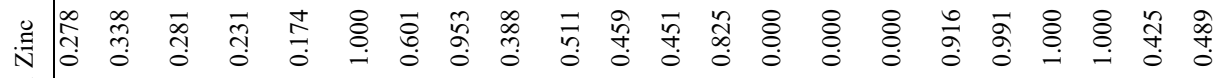

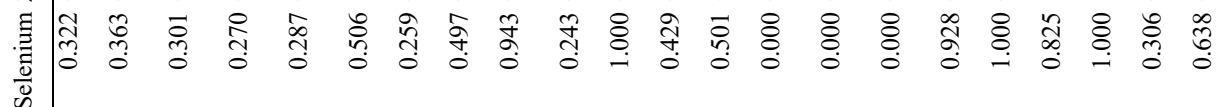

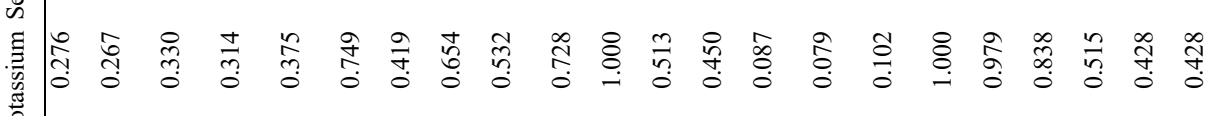

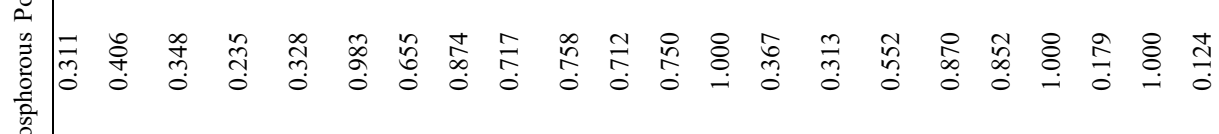

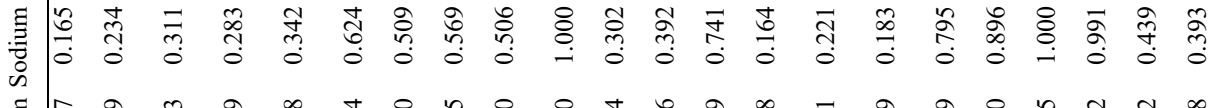

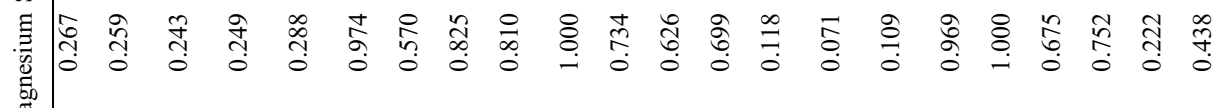

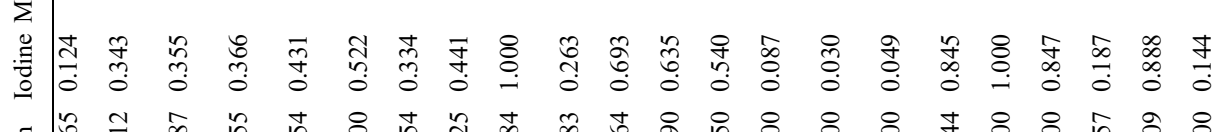

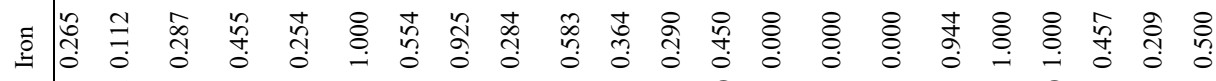

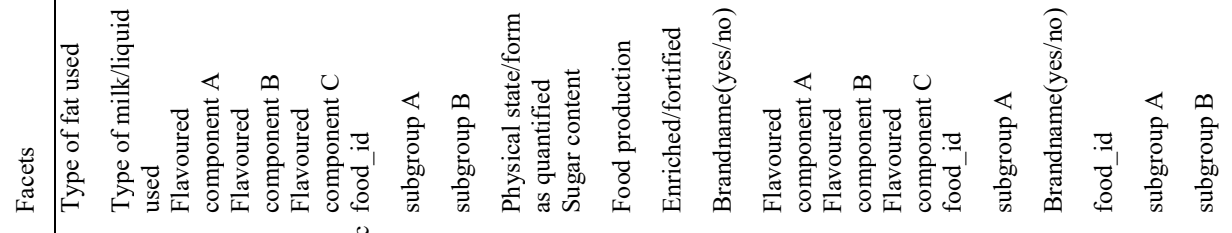

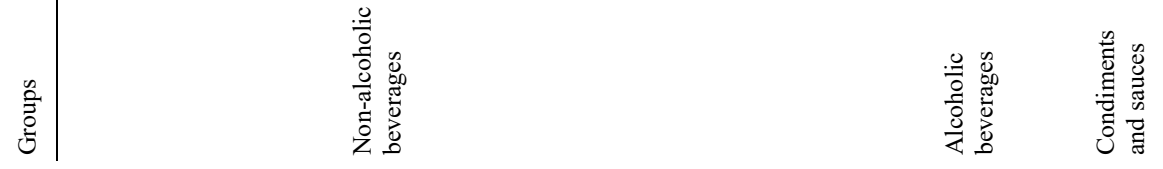




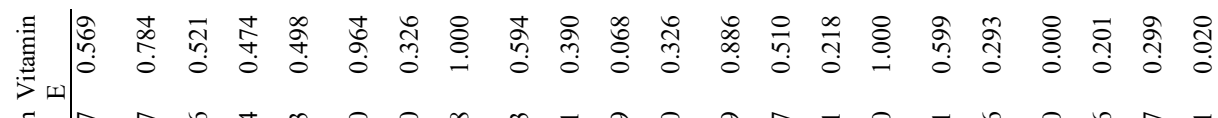

皇

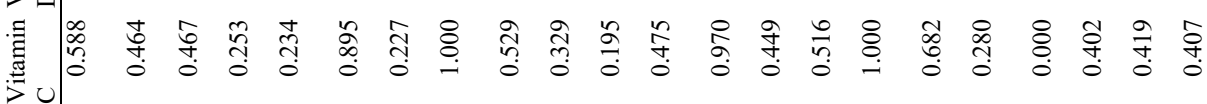

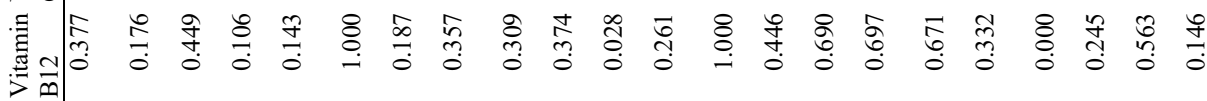

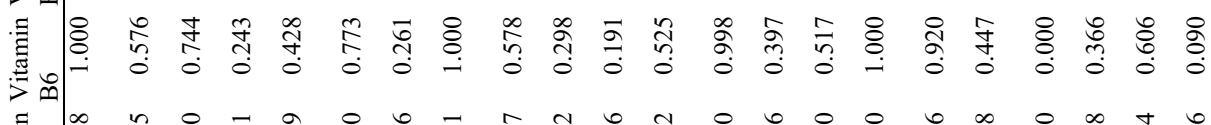

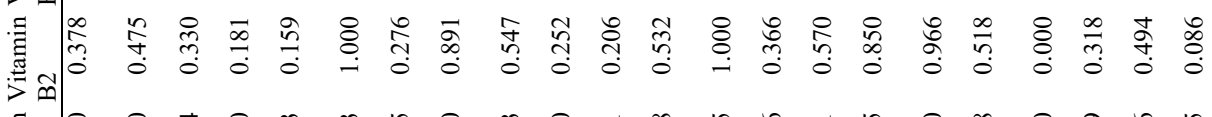

言

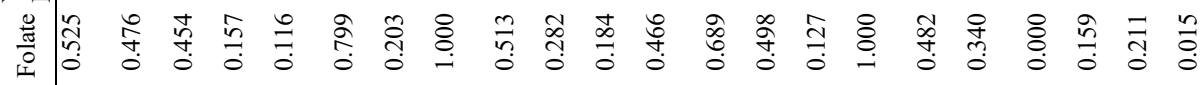

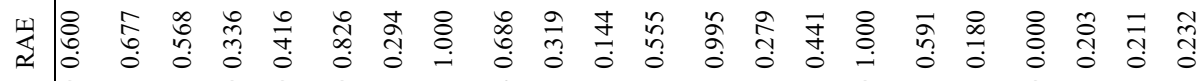

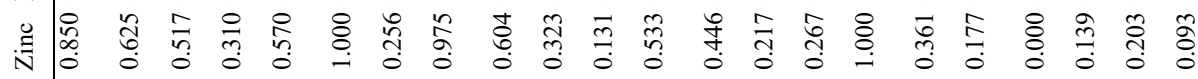

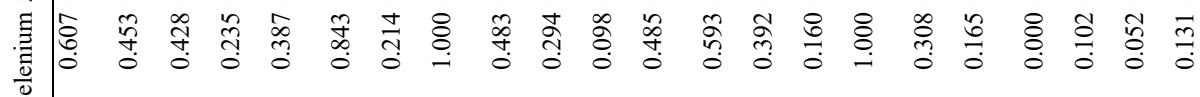

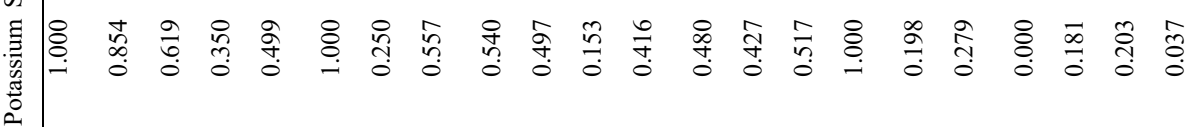

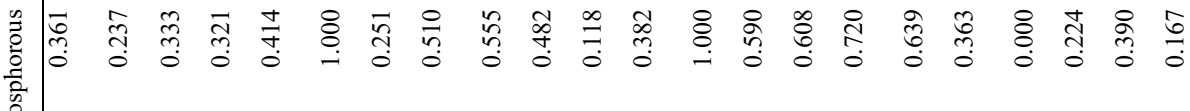

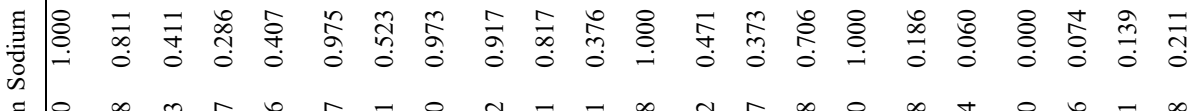

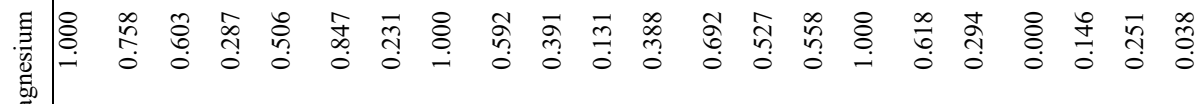

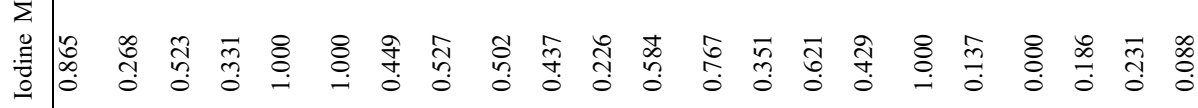

洜全

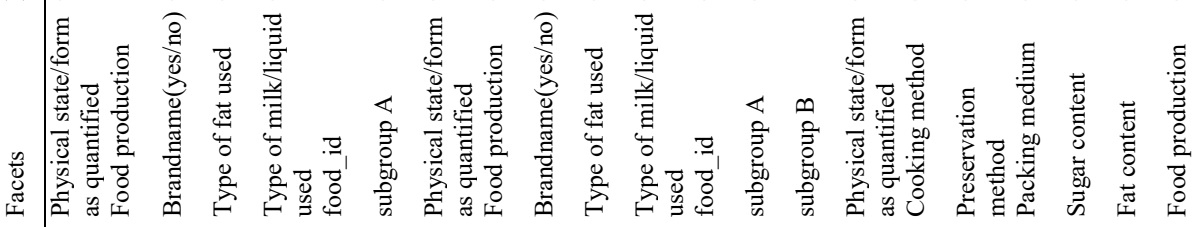


76 | Chapter 2

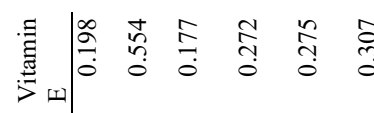

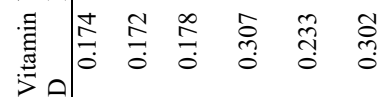

息

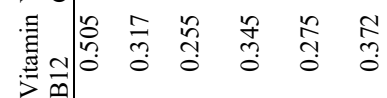

晹

立

㫷

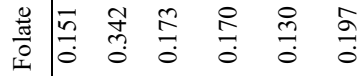

尘

苞

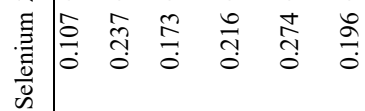

言

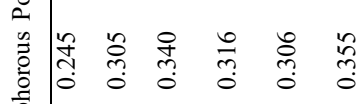

离

索

毒

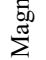

寻

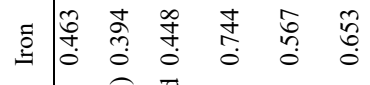

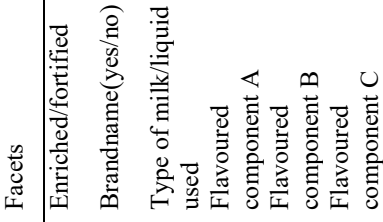

产 
تே

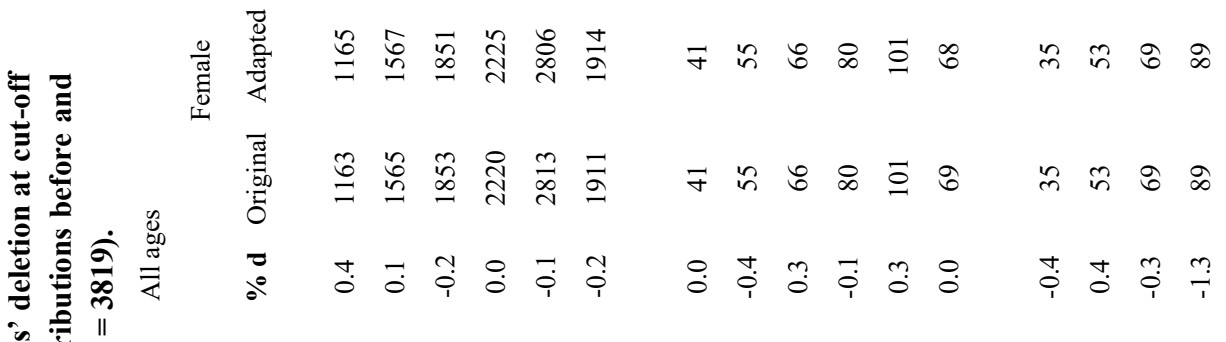

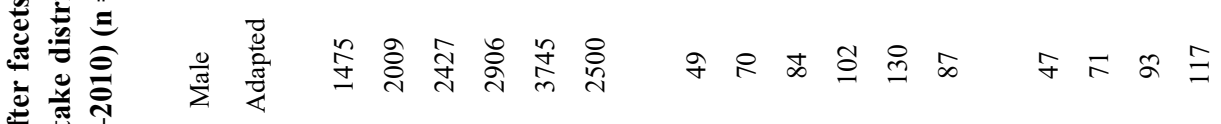

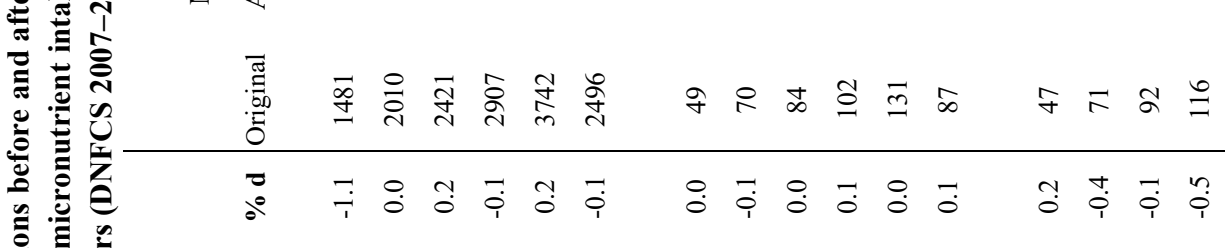

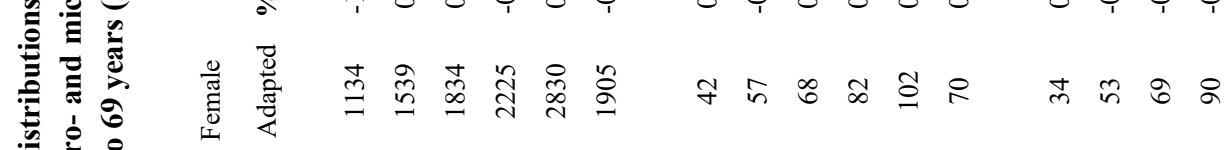

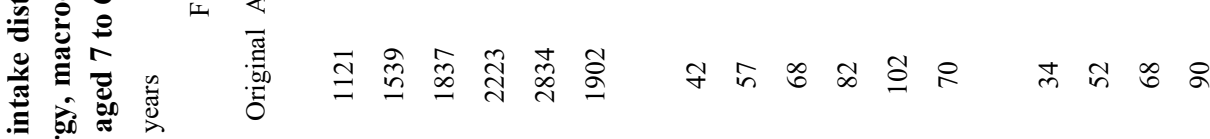

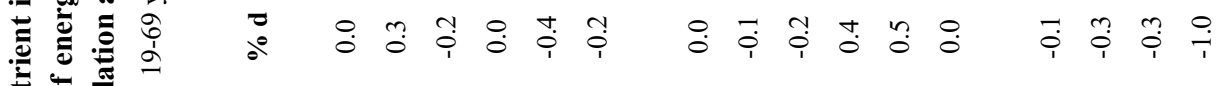

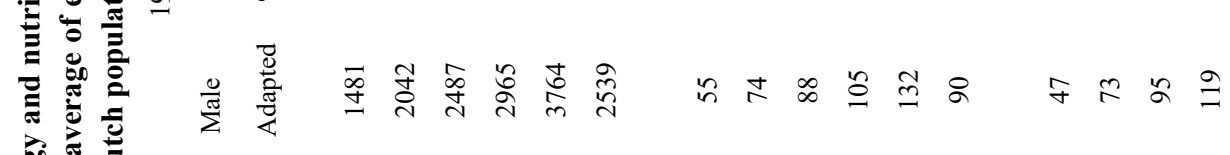

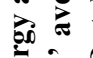

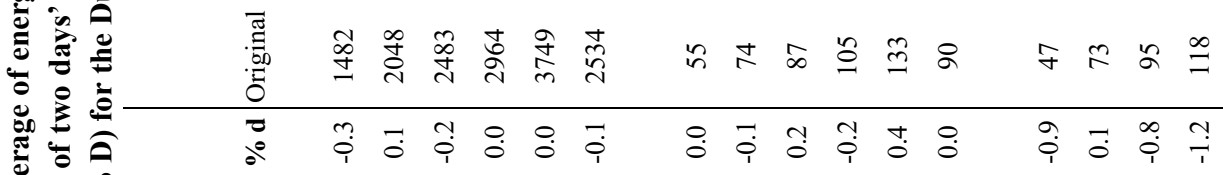

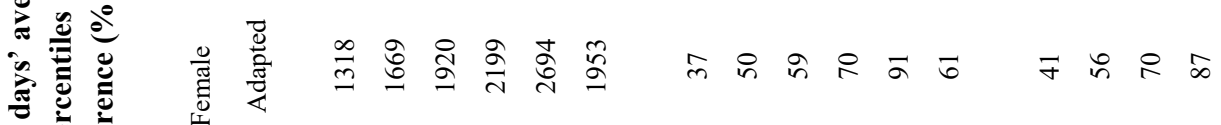

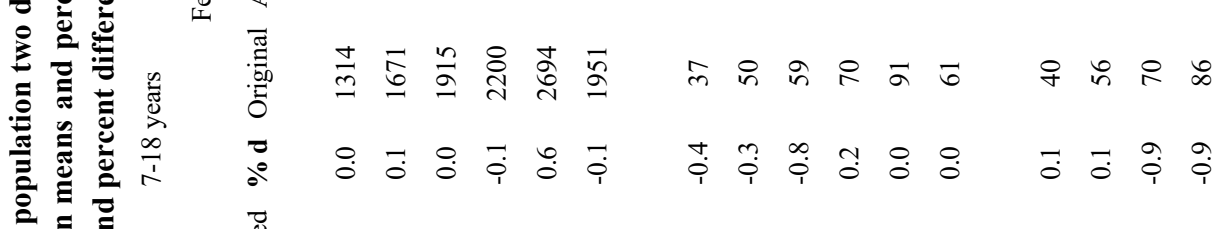

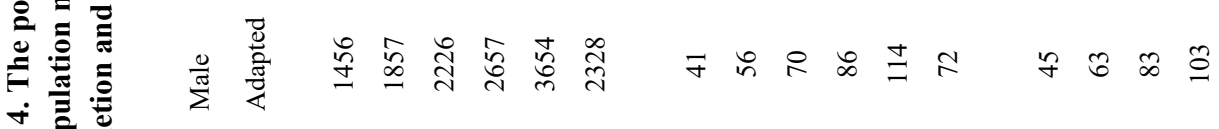

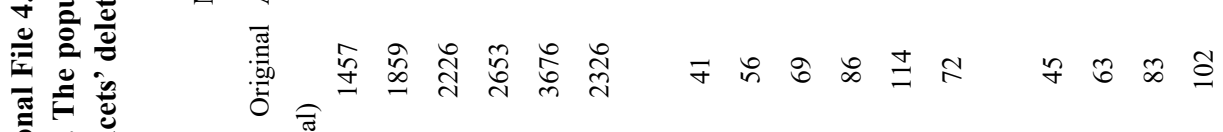

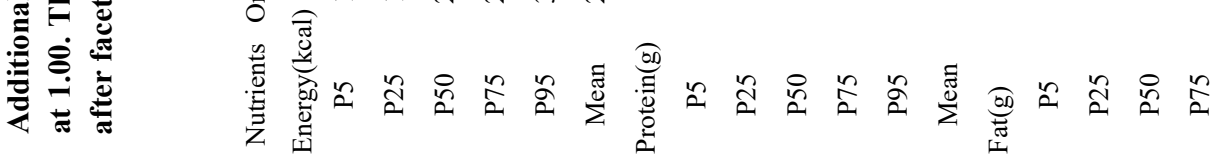




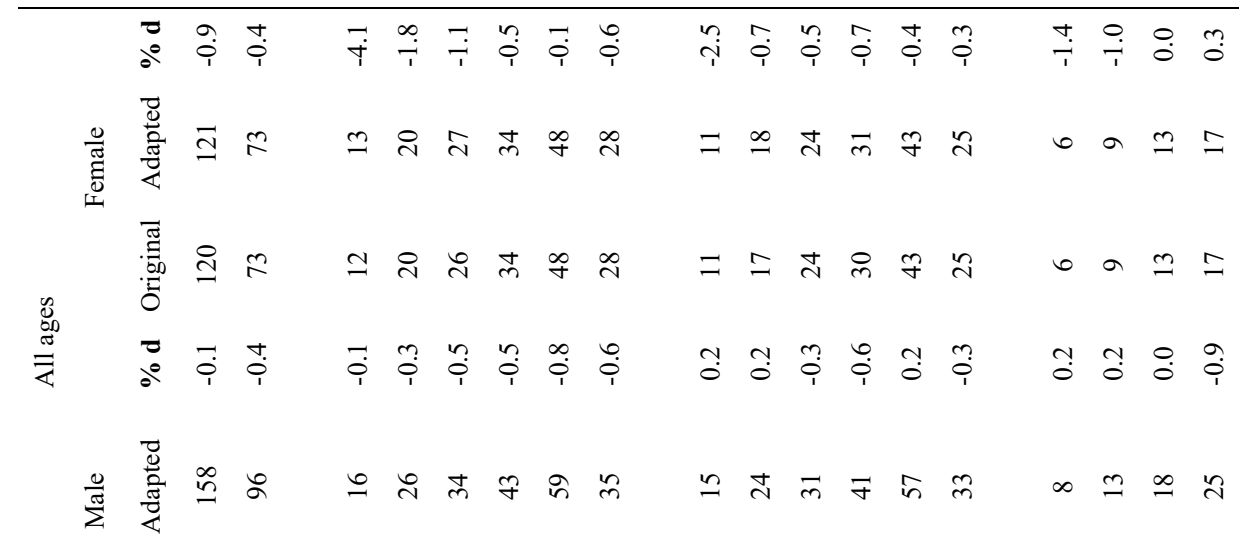

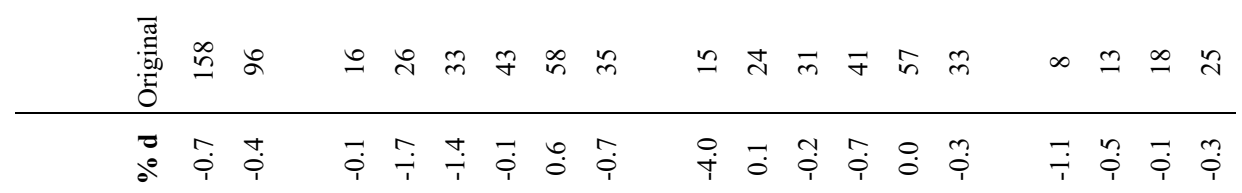
苛

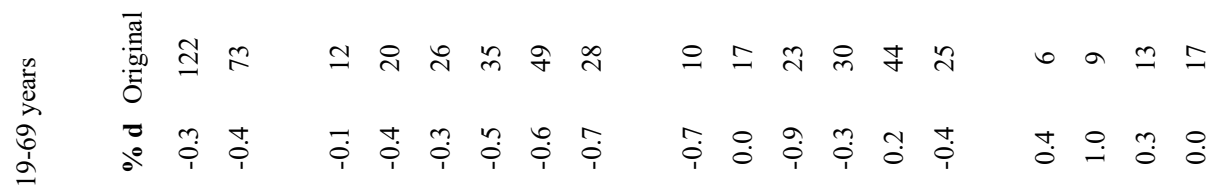

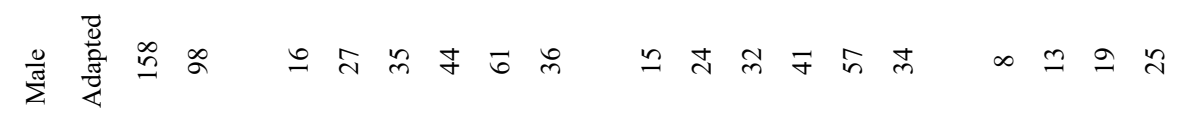

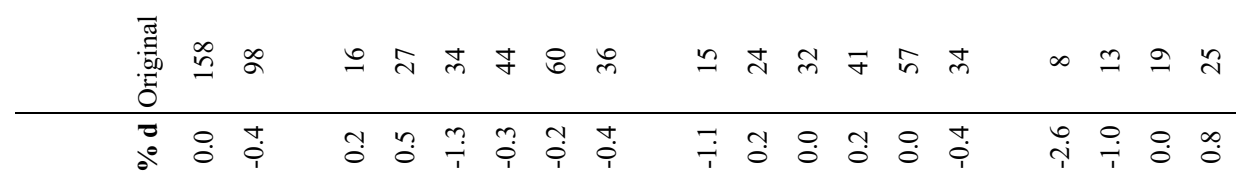

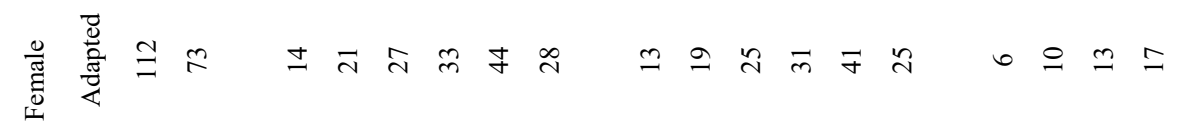

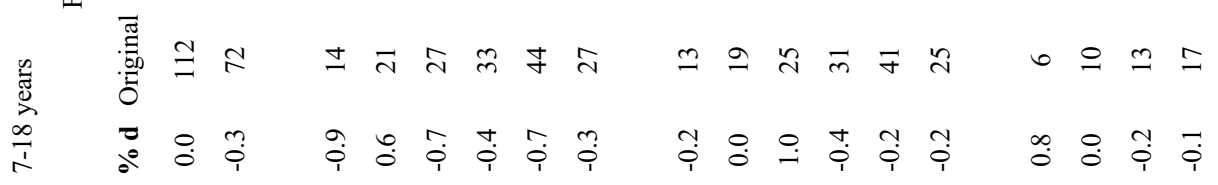
造莺 节

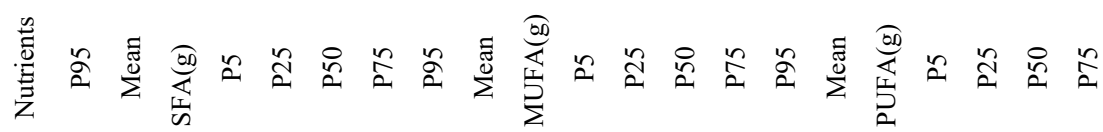




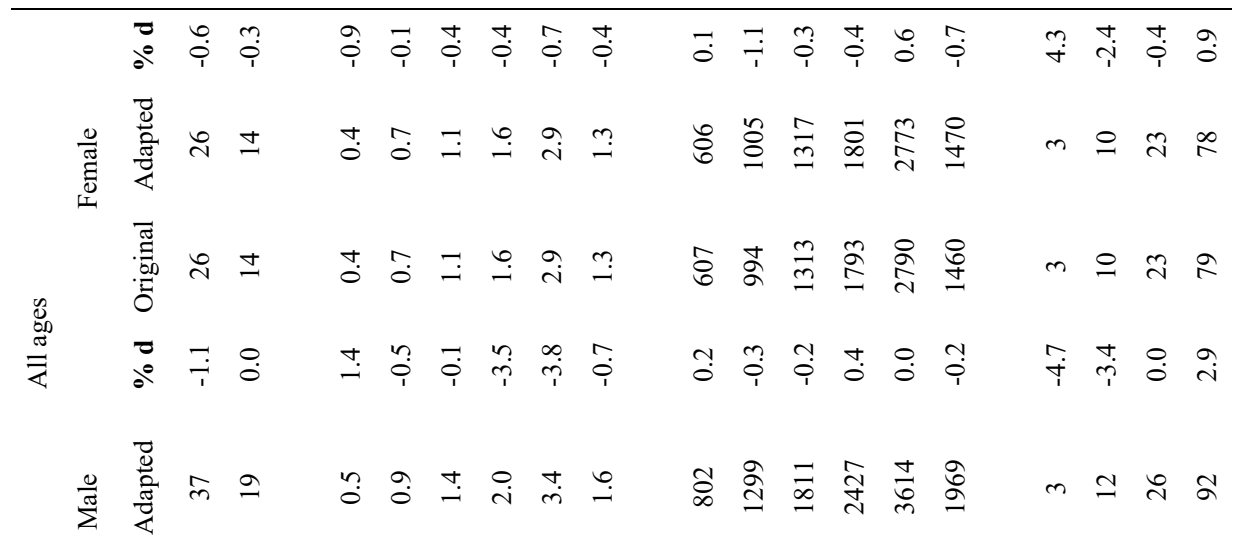

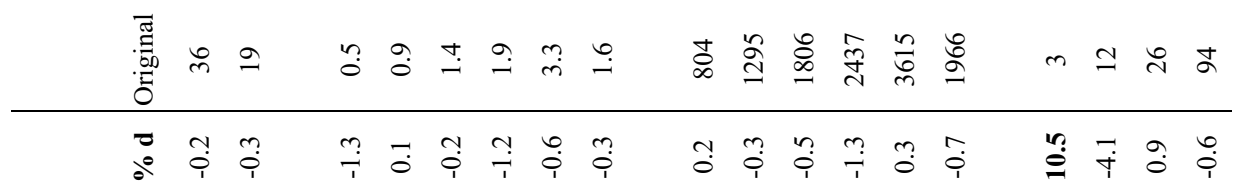

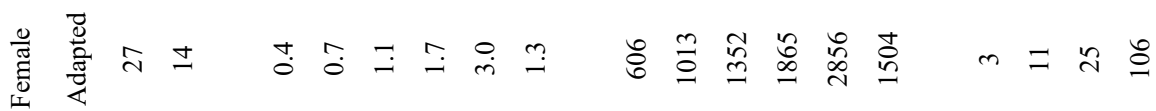

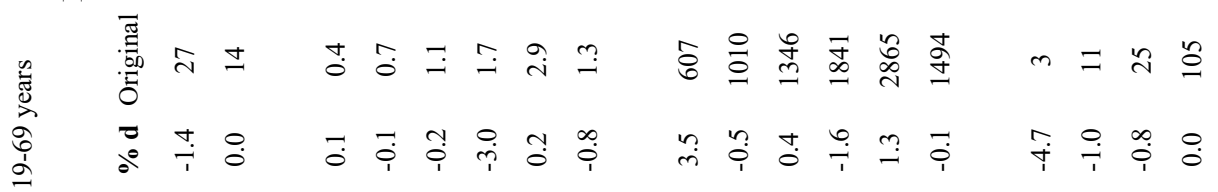

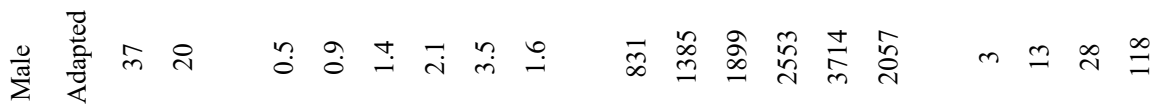

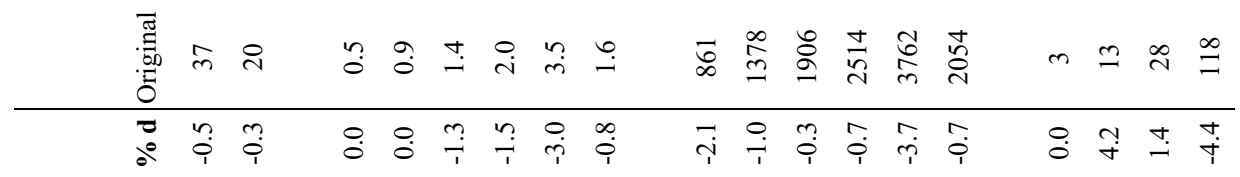

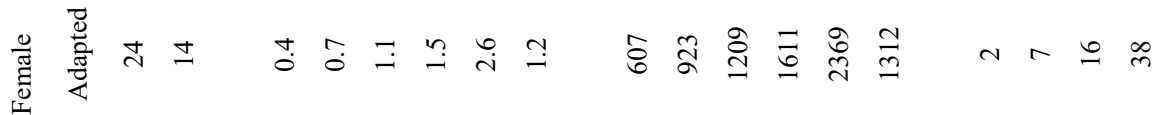
营

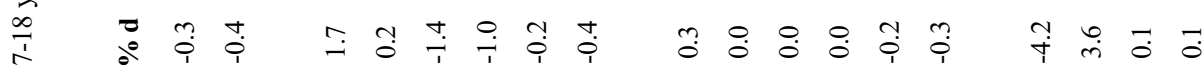

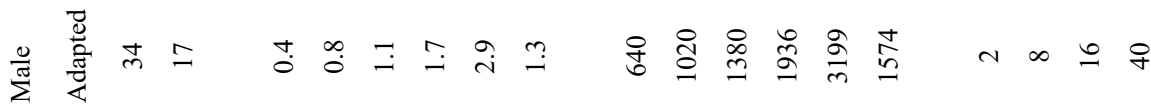
䔍 产 


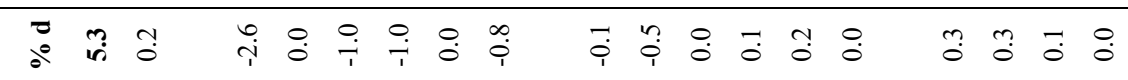

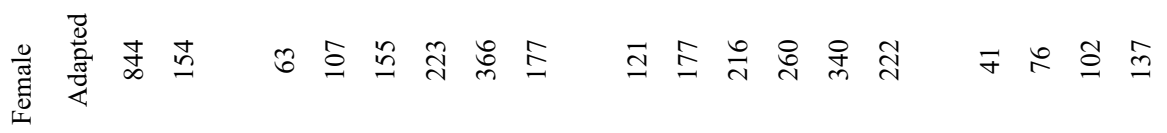

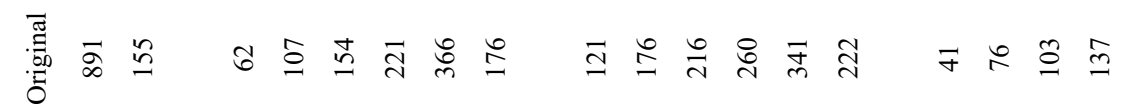

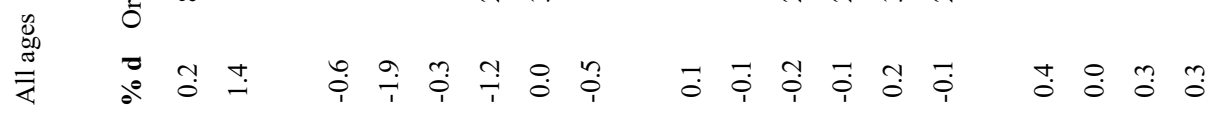

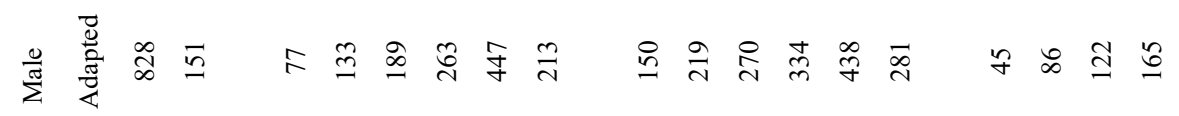

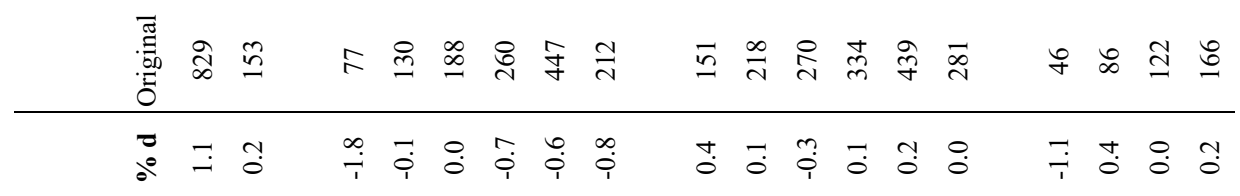

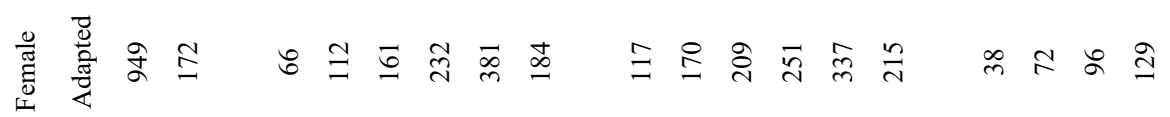

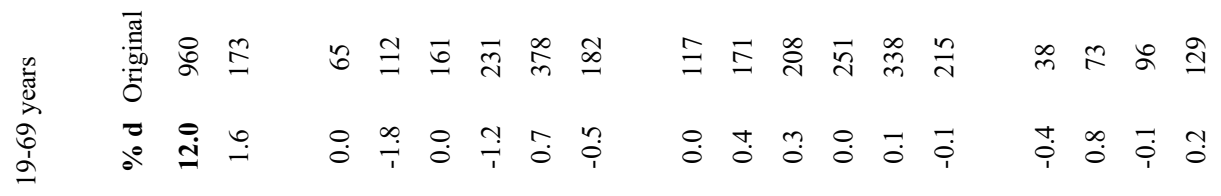

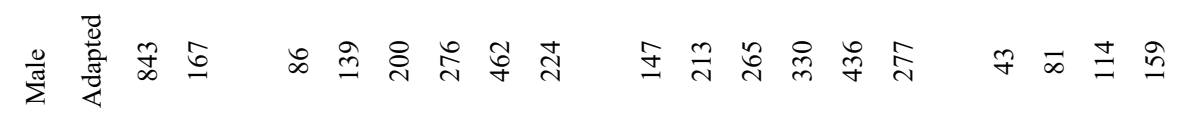

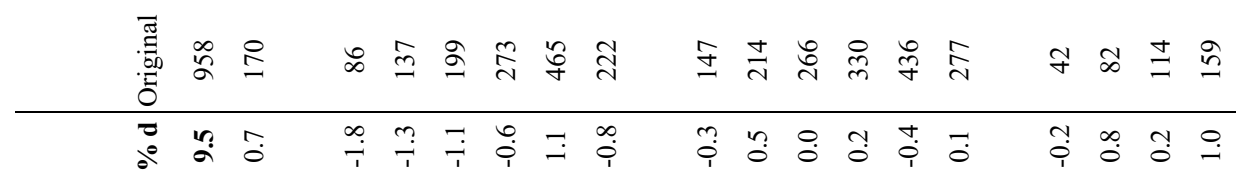

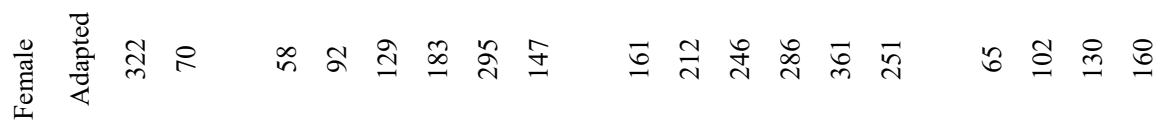

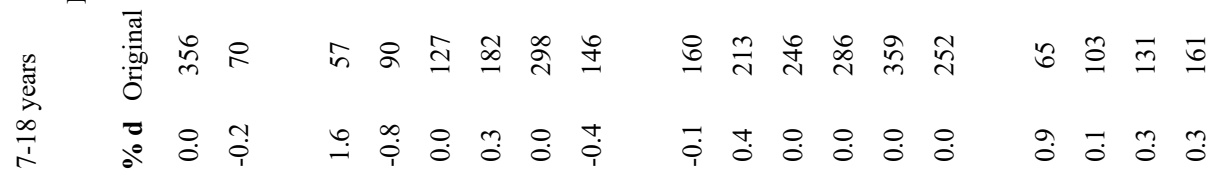

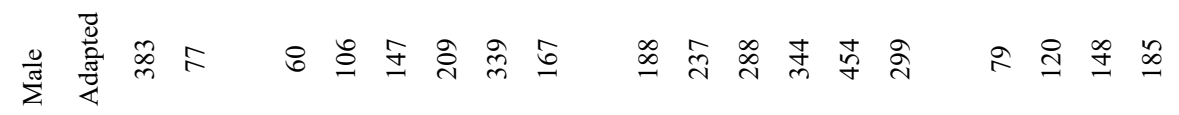

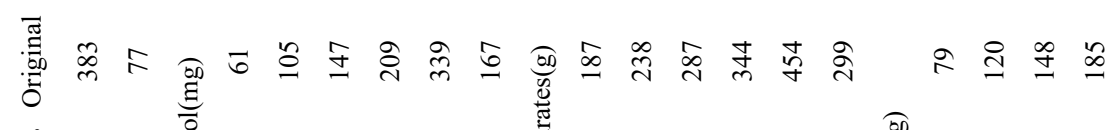

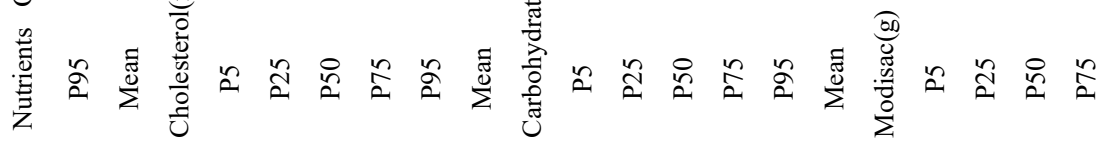




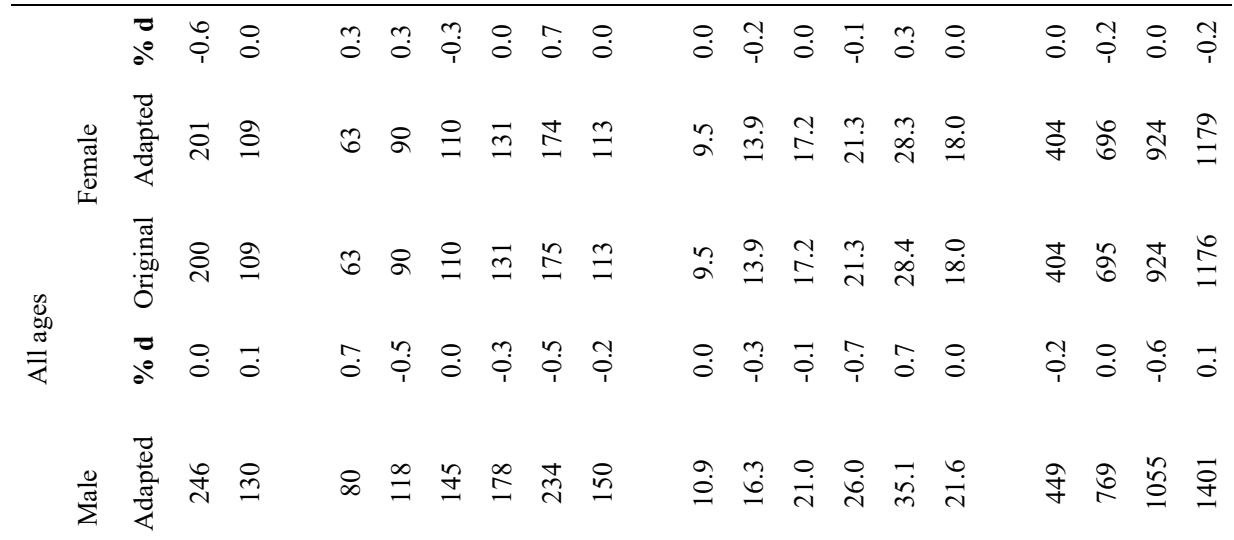

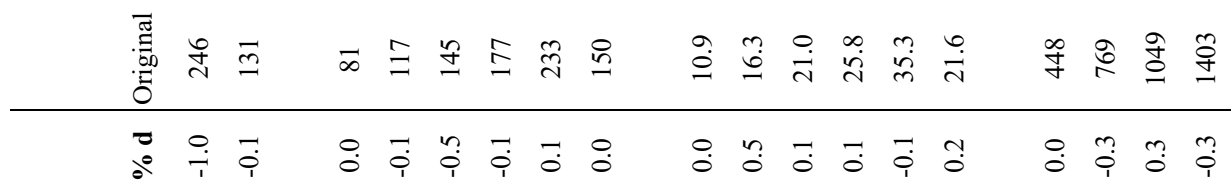

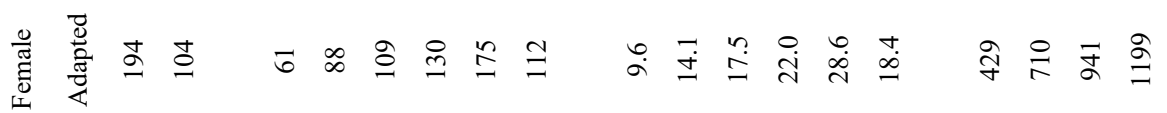

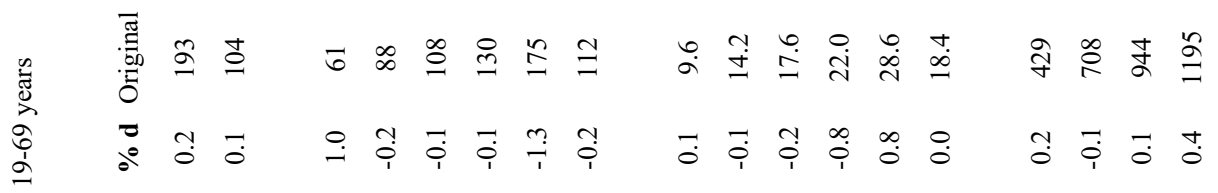

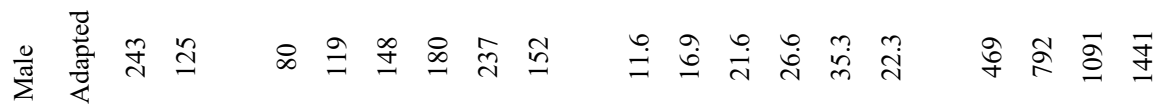

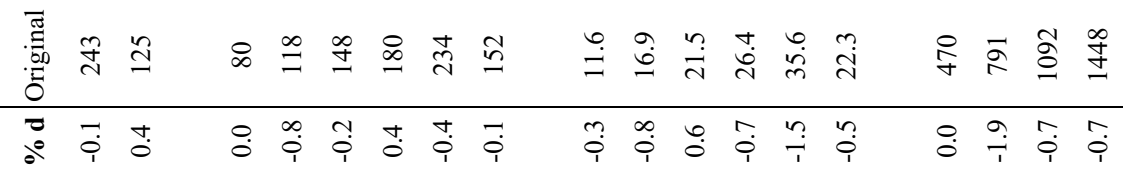

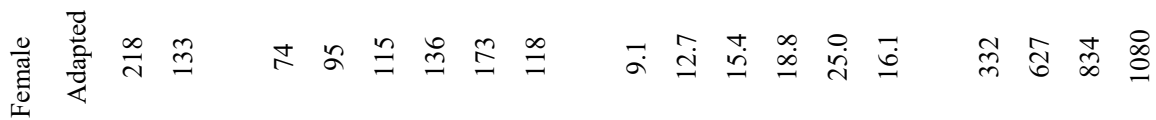

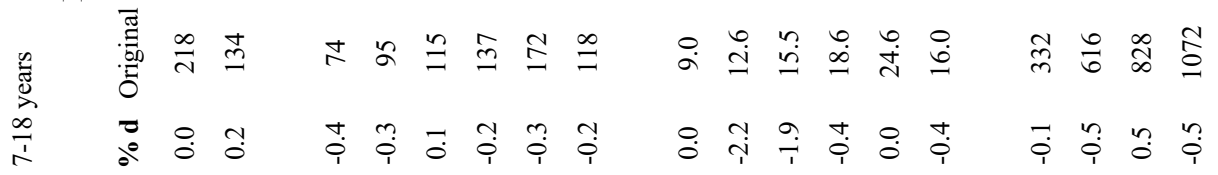

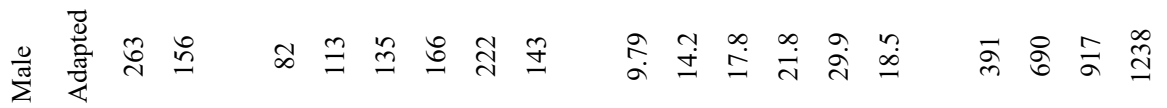
焉

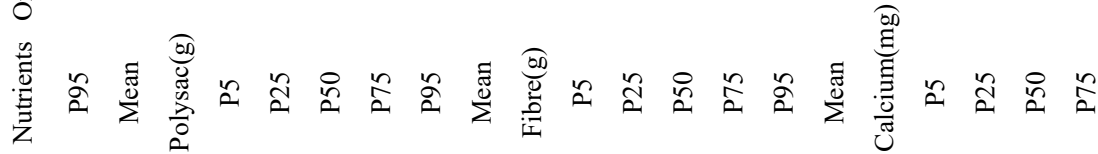




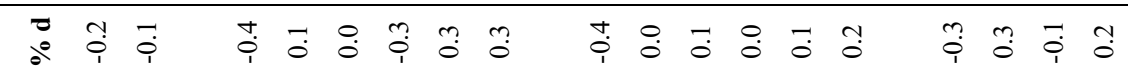

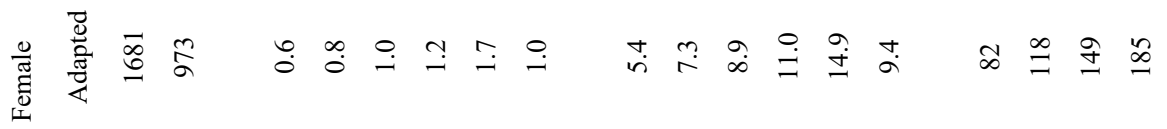

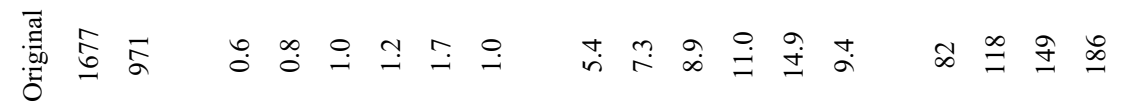
展

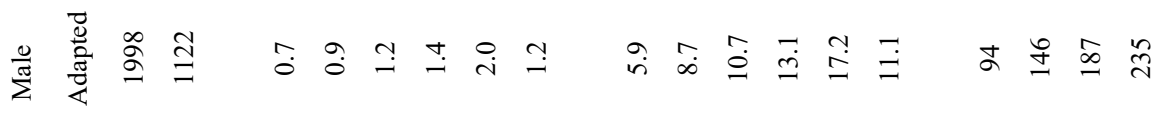

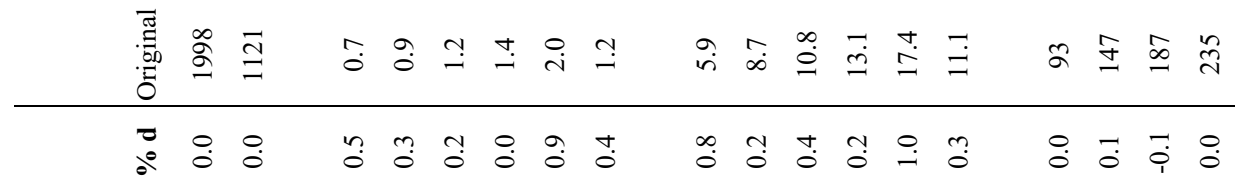

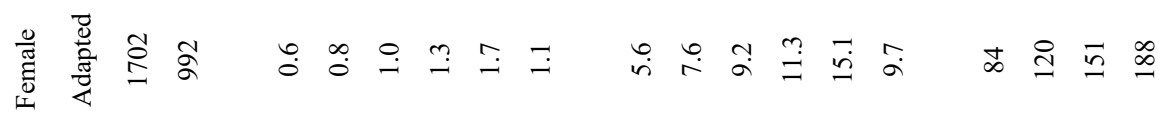

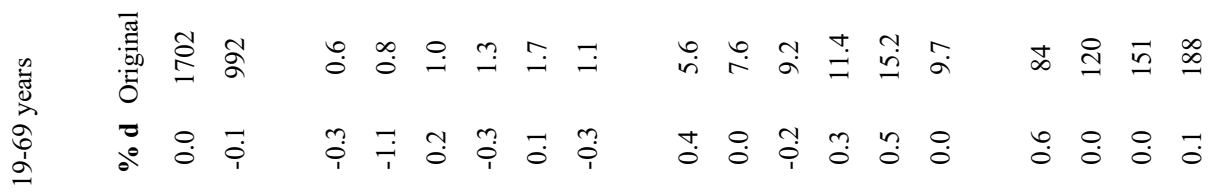

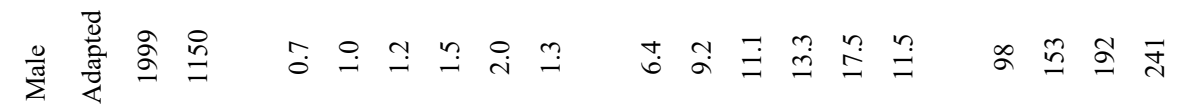

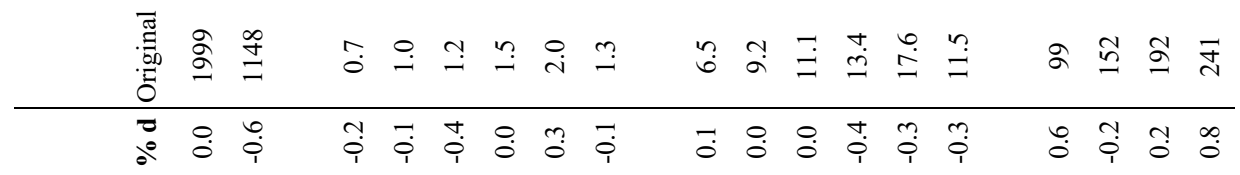

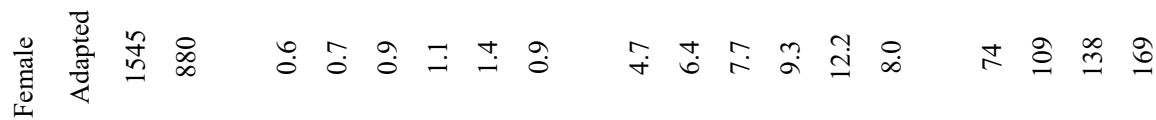

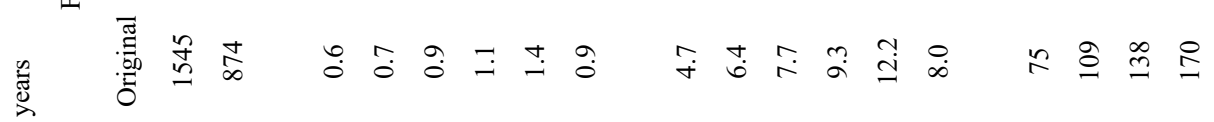

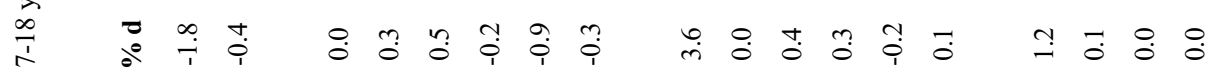

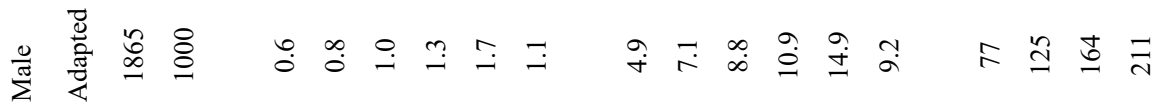

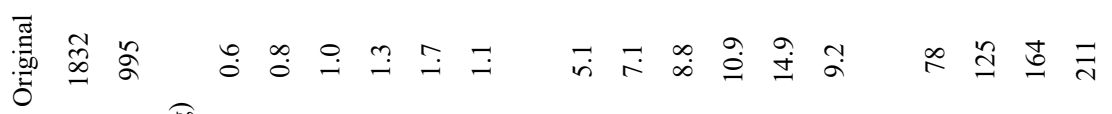

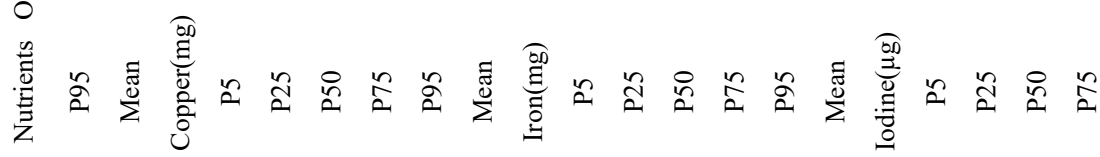




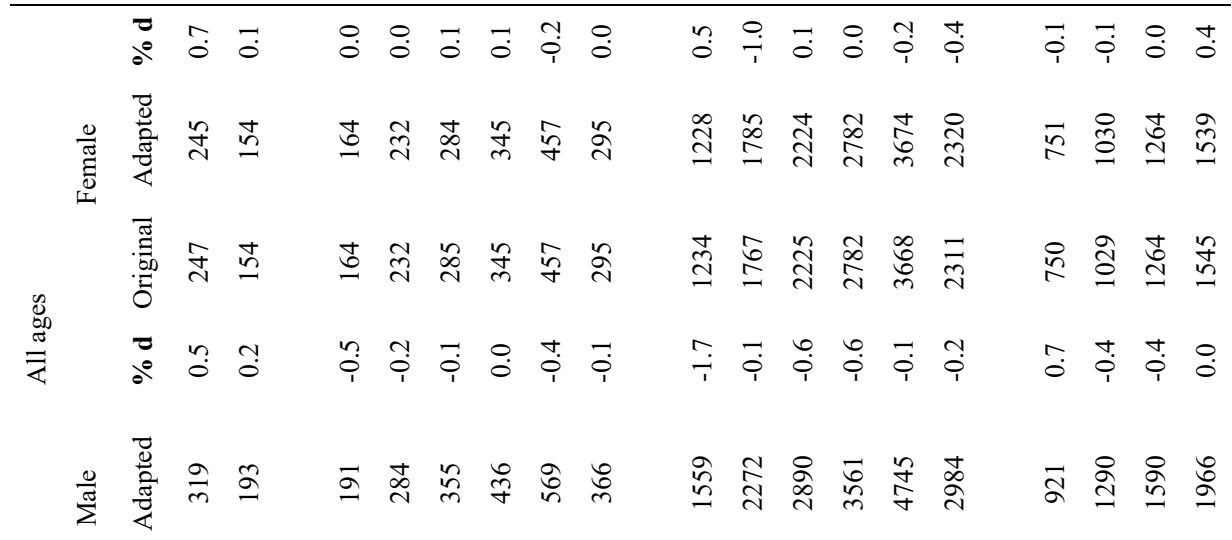

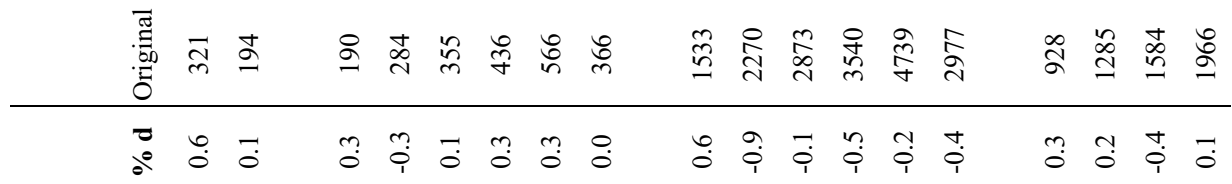

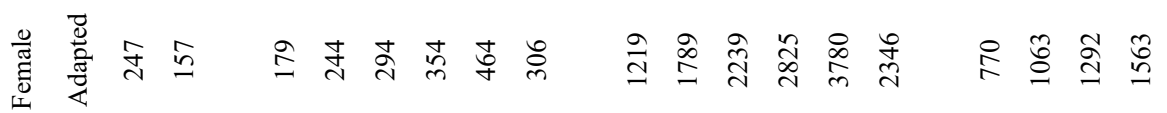

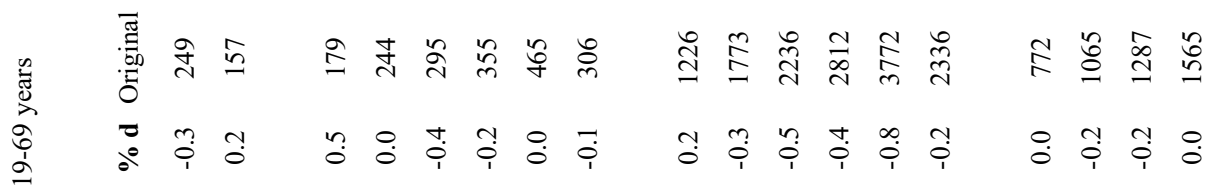

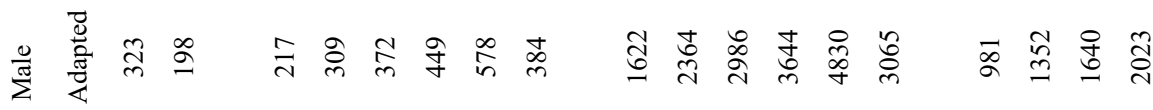

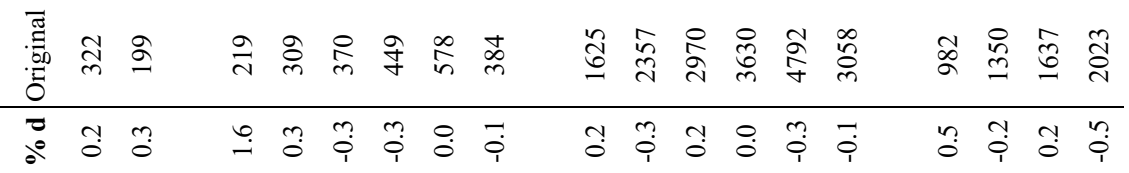

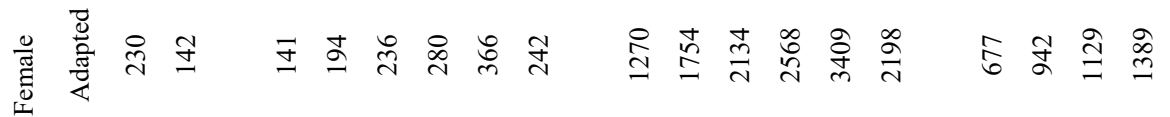

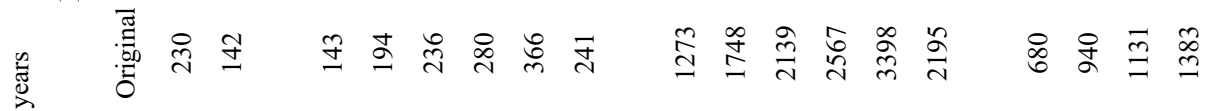

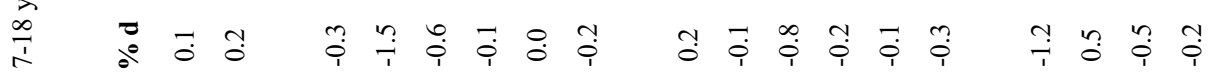

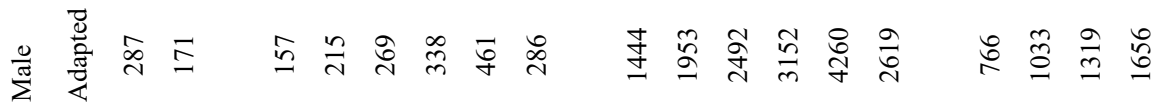

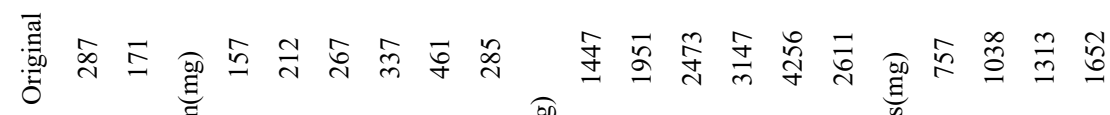

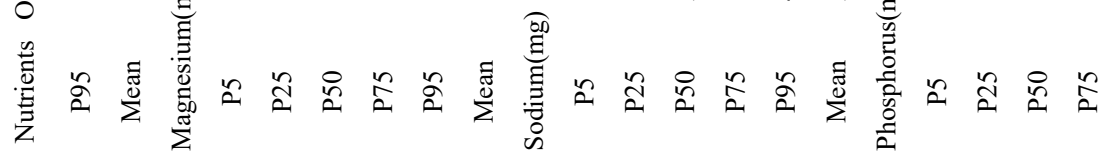




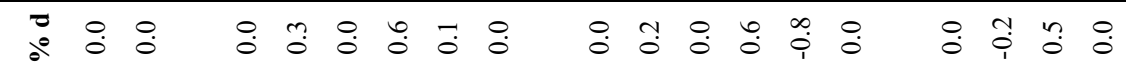

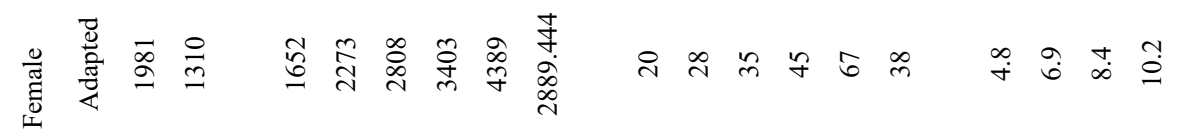

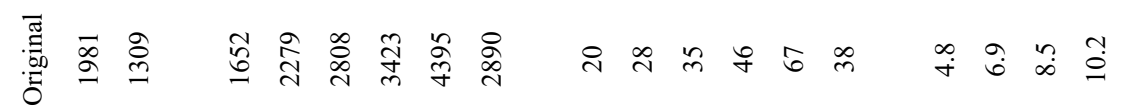

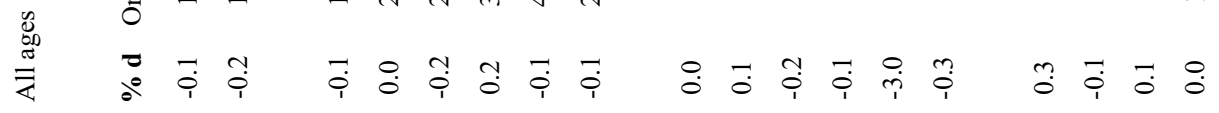

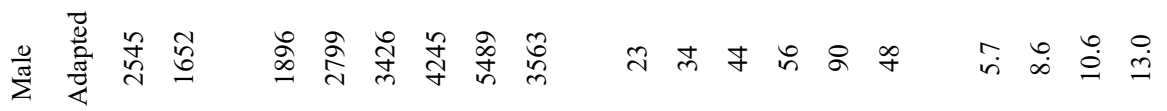

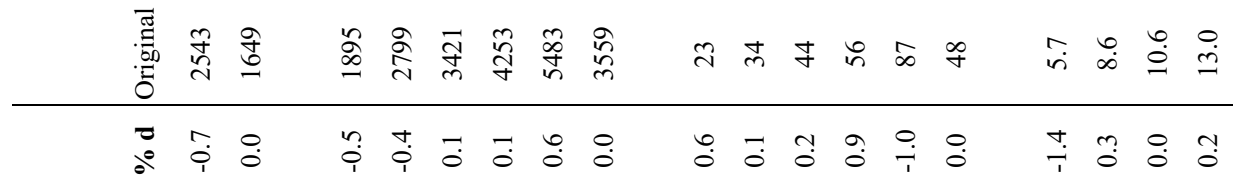

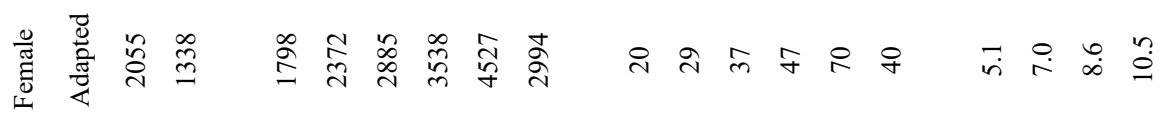

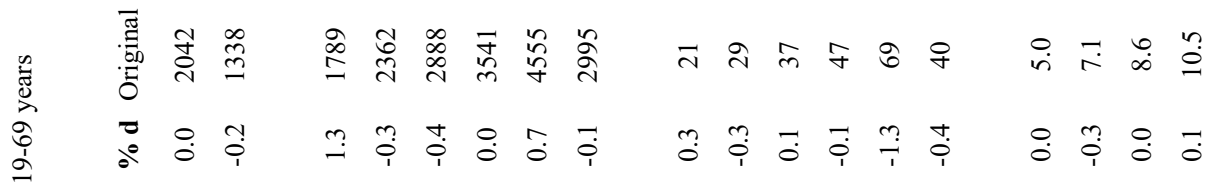

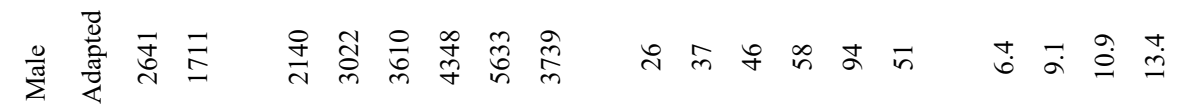

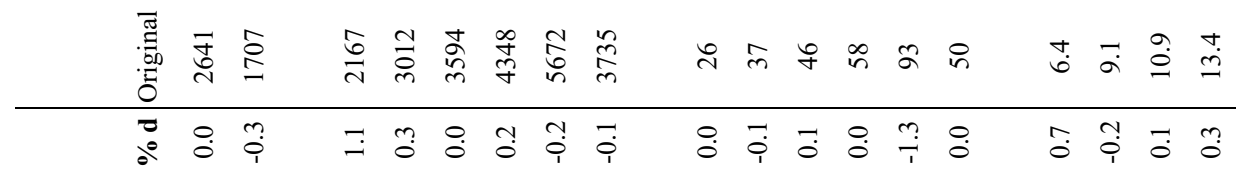

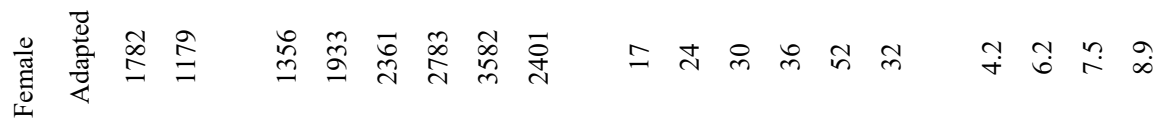

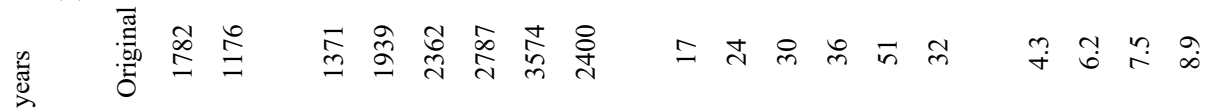

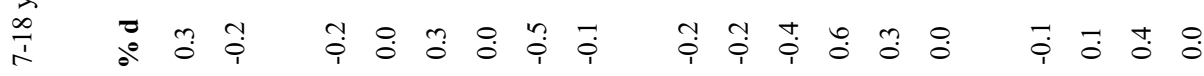

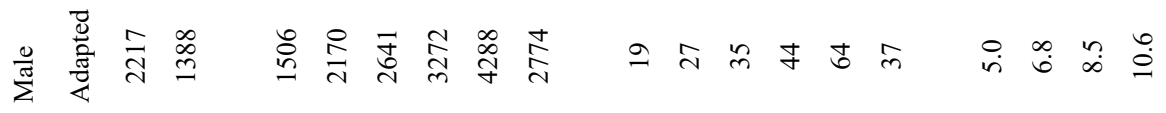

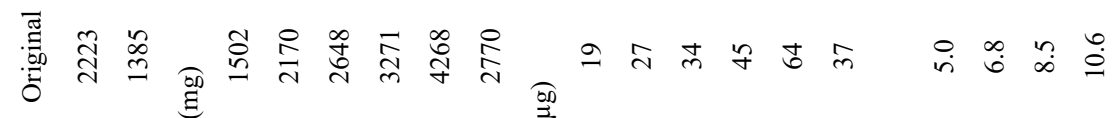

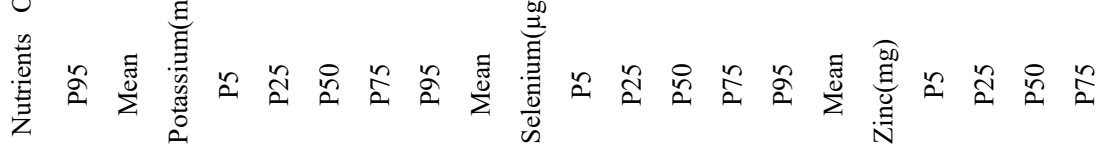




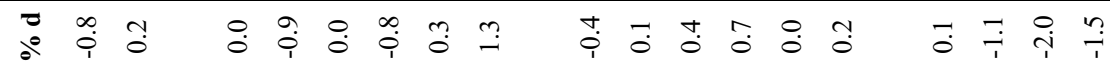

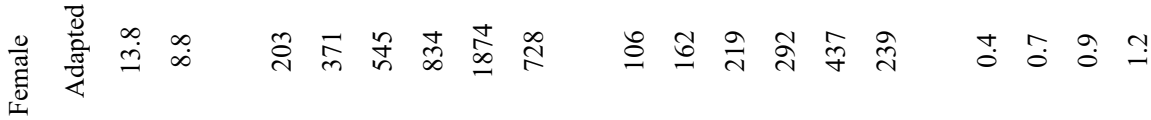

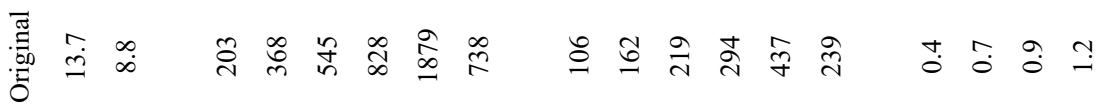
总

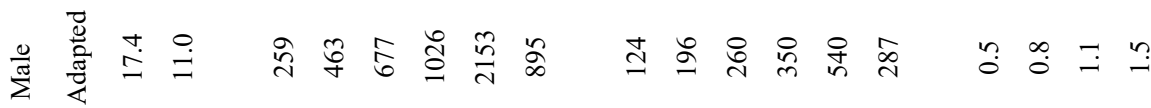

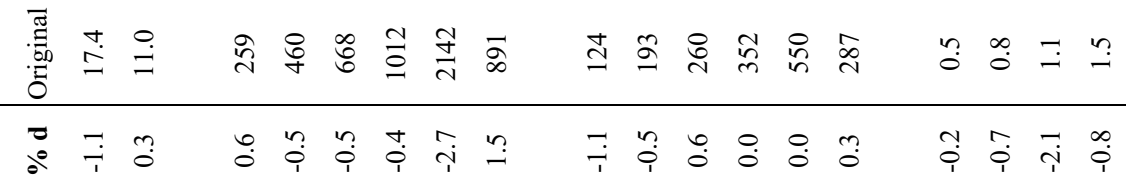

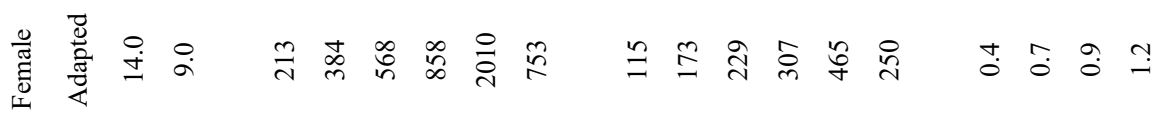

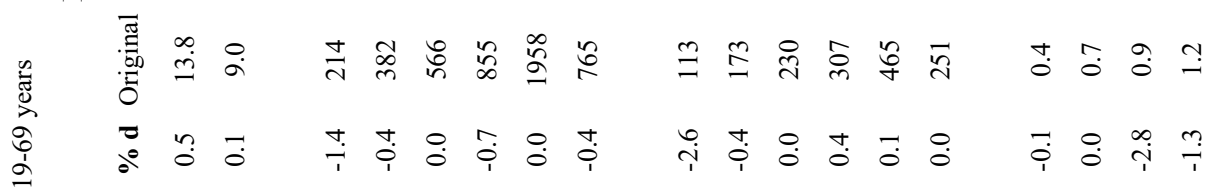

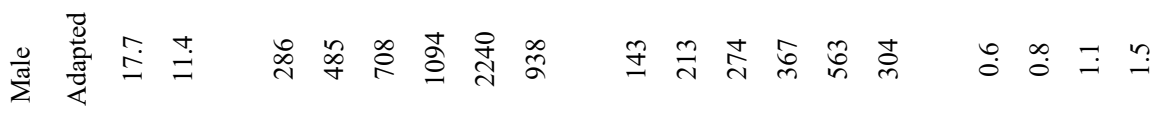

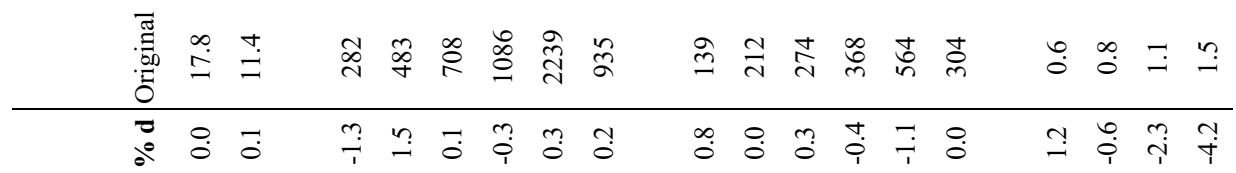

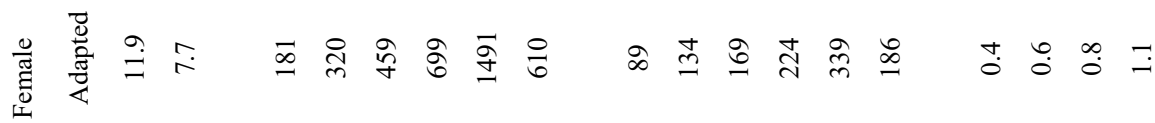

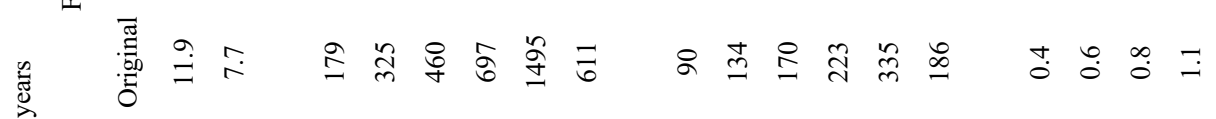

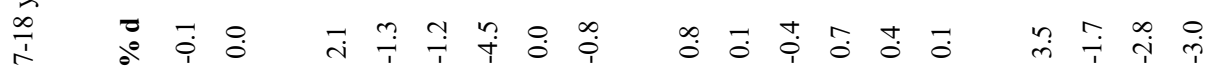

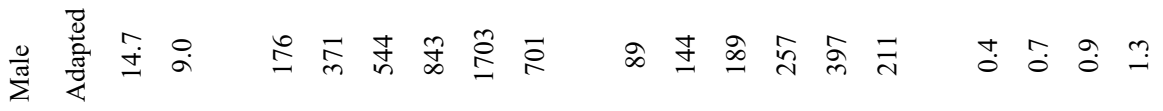

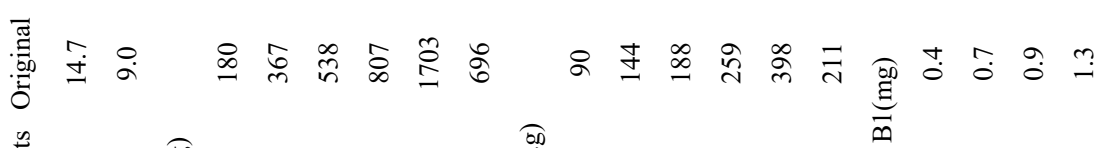

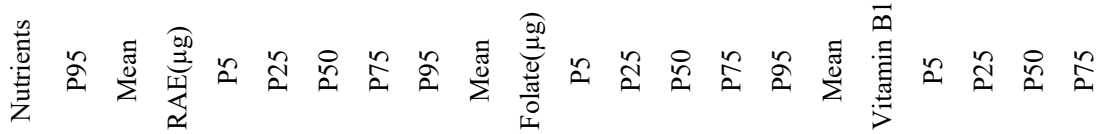




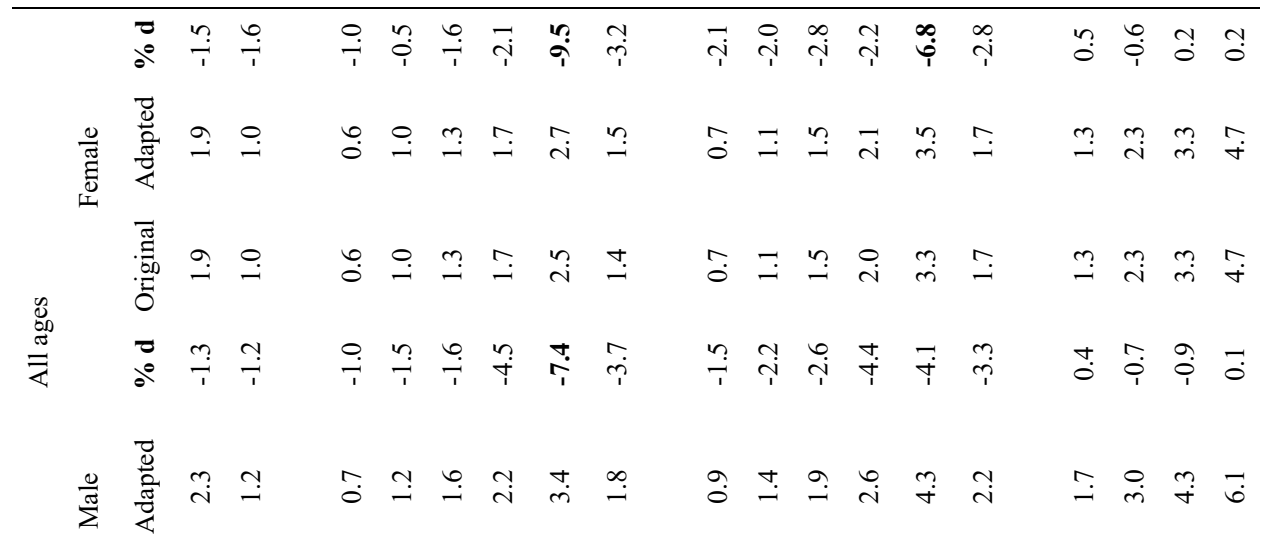

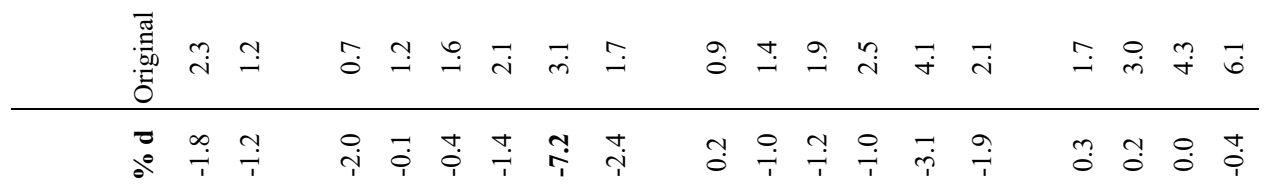
苞

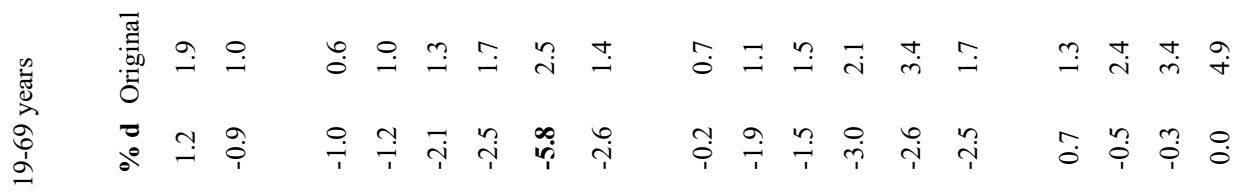

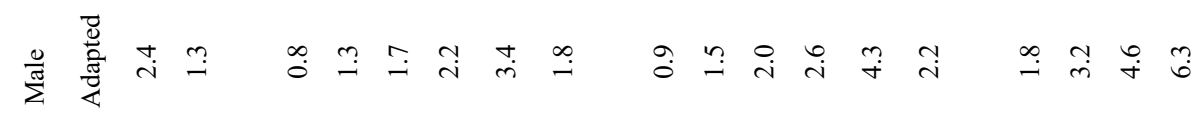

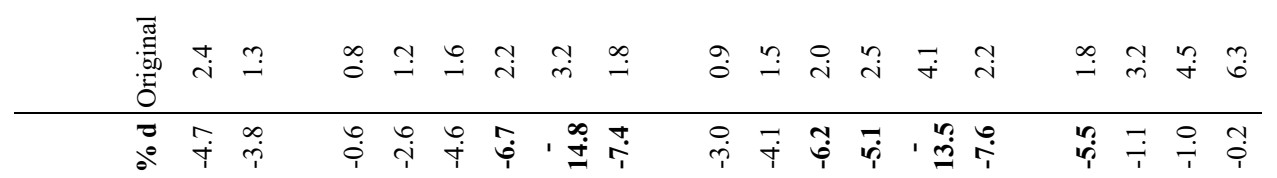

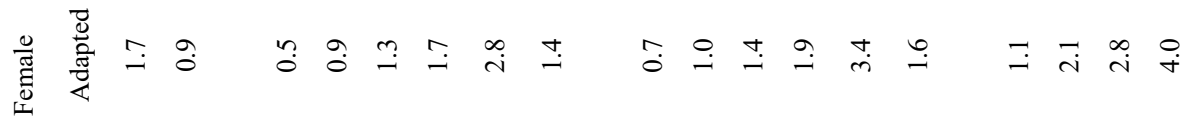

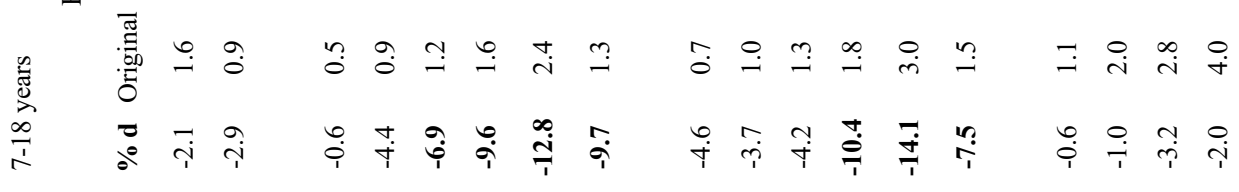
选 营

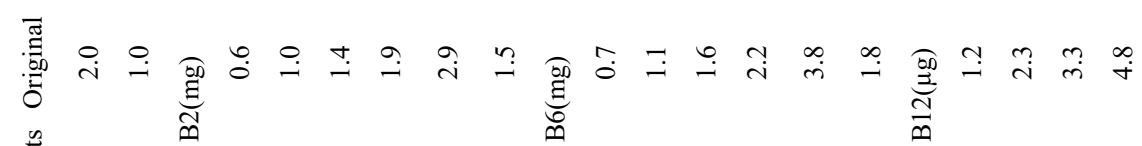

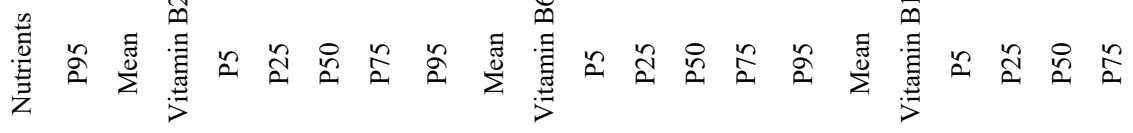




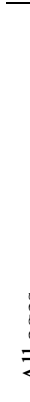

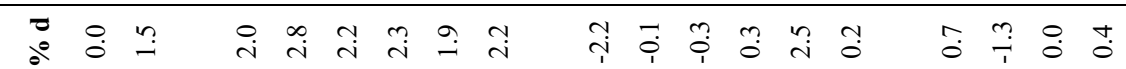

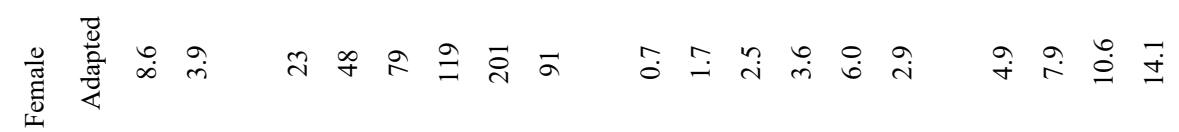

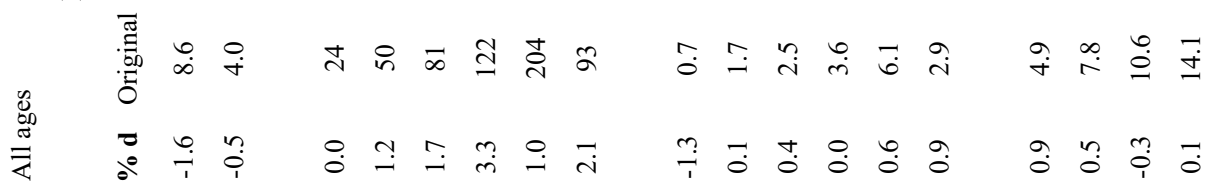

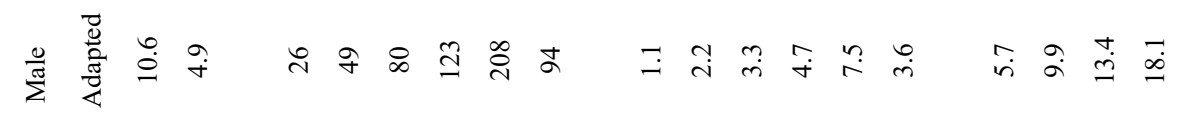

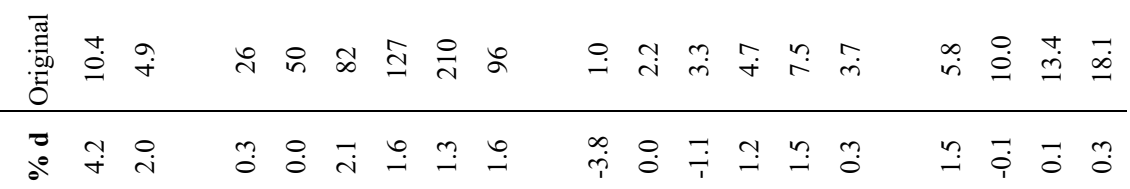

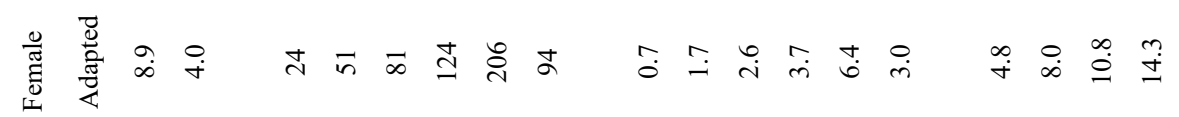

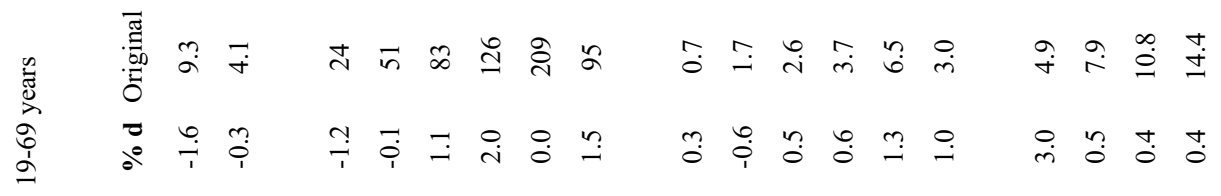

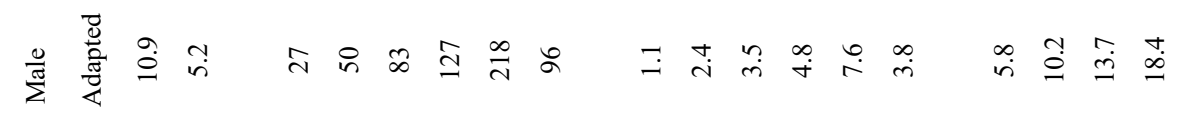

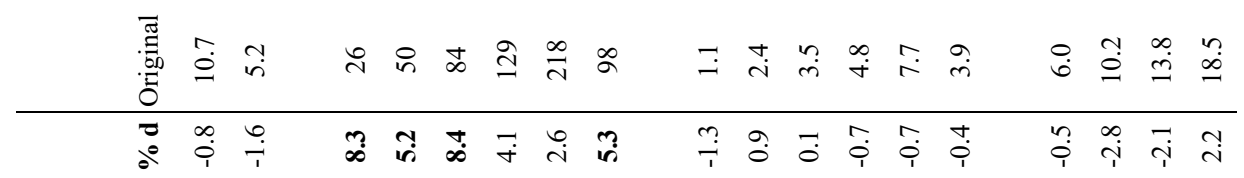

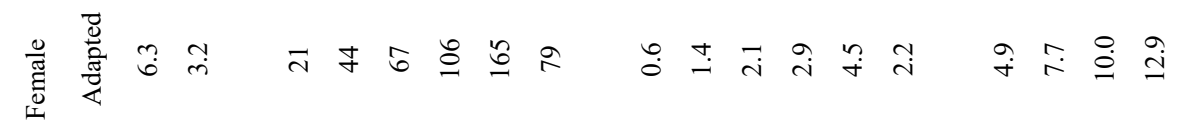

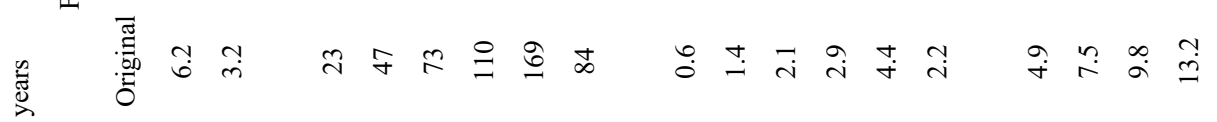

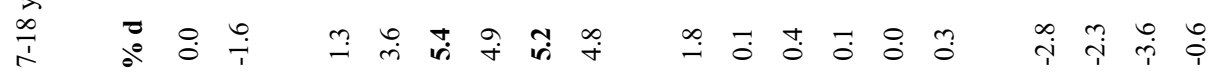

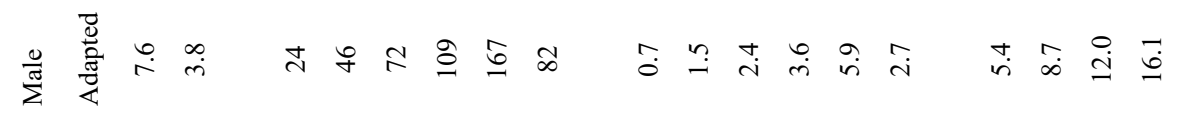

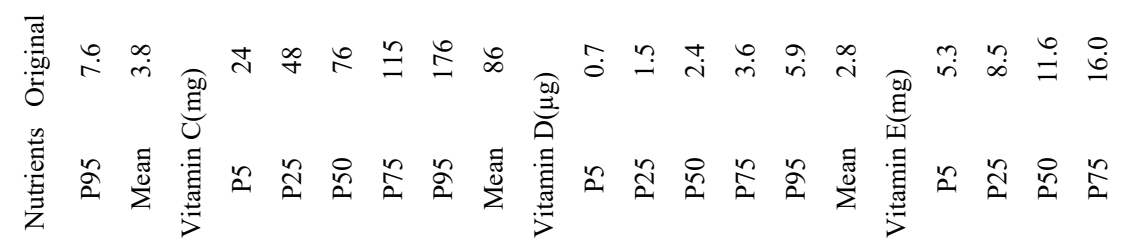


Chapter 2

$$
\begin{aligned}
& \text { ¿ }
\end{aligned}
$$

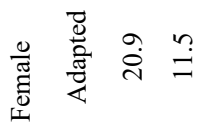

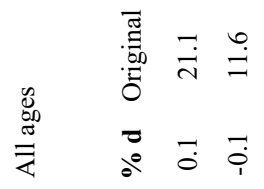

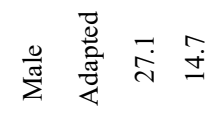

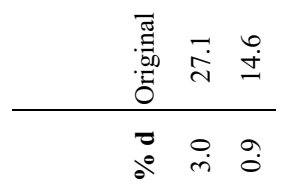

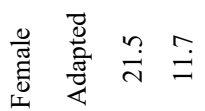

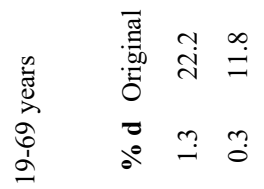

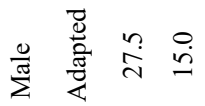

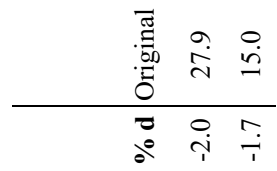

$$
\begin{aligned}
& \text { 总 }
\end{aligned}
$$

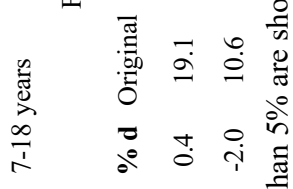

$$
\begin{aligned}
& \text { 芯 营 完 } \\
& \text { 莺 }
\end{aligned}
$$

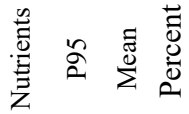






\section{Chapter 3}

How Does a Simplified Recipe

Collection Procedure in Dietary

Assessment Tools Affect the

Food Group and Nutrient Intake

Distributions of the Population

Liangzi Zhang, Hendriek Boshuizen, Marga Ocké

British Journal of Nutrition 2020, 124(2), 189-198 


\begin{abstract}
Technology advancements have driven the use of self-administered dietary assessment methods in large-scale dietary surveys. Interviewer-assisted methods generally have a complicated recipe recording procedure enabling the adjustment from a standard recipe. In order to decide if this functionality can be omitted for self-administered dietary assessment, this study aimed to assess the extent of standard recipe modifications in the Dutch National Food Consumption Survey, and measure the impact on the food group and nutrient intake distributions of the population when the modifications were disregarded. A two-scenario simulation analysis was conducted. Firstly, the individual recipe scenario omitted the full modifications to the standard recipes made by people who knew their recipes. Secondly, the modified recipe scenario omitted the modifications made by those who partially modified the standard recipe due to their limited knowledge. The weighted percentage differences for the nutrient and food group intake distributions between the scenarios and the original dataset were calculated. The highest percentage of energy consumed through mixed dishes was $10 \%$ for females aged 19 to 79. Comparing the combined scenario and the original dataset, the average of the absolute percentage difference for the population mean intakes was $1.6 \%$ across all food groups and $0.6 \%$ for nutrients. The soup group (-6.6\%) and docosahexaenoic acid (DHA) (-2.3\%) showed the largest percentage difference. The recipe simplification caused a slight underestimation of the consumed amount of both foods $(-0.2 \%)$ and nutrients $(-0.4 \%)$. These results are promising for developing self-administered $24 \mathrm{hR}$ or food diary applications without complex recipe function.
\end{abstract}




\section{Introduction}

Inappropriate dietary intakes have been recognized as major risk factors for developing chronic diseases ${ }^{(1,2)}$. Many countries, therefore, carry out national food consumption surveys to monitor food consumption and nutrient intakes of their populations ${ }^{(3)}$. The most frequently used dietary assessment methods in Europe for collecting national food consumption data are 24-hour-recalls (24hRs) and food records ${ }^{(4)}$, both open methods aim to assess the intake of all foods and drinks on a specific day(s). 24hRs require low literacy levels of participants and are less likely to alter eating behaviours than food records ${ }^{(5,6)}$, whereas food records have less recalling bias $^{(7)}$. To collect harmonised data among the EU Member states, the European Food Safety Authority recommended collecting two non-consecutive $24 \mathrm{hRs}$ for adults and two non-consecutive food records for children. Moreover, the use of validated and standardized software was advised, for example, GloboDiet (formerly known as EpicSoft $)^{(8,9,10)}$. The EFSA guidelines were based on the experiences and recommendations from various European projects, such as the EFCOSUM-project ${ }^{(11)}$, the EFCOVAL project $^{(12)}$, the PANCAKE project ${ }^{(13)}$ and the PAN-EU project ${ }^{(14)}$.

Although detailed food consumption information can be captured, the current intervieweradministered dietary assessment method induces high costs and logistic complications for data collection and handling ${ }^{(15,16)}$. This limitation encourages efforts to explore solutions that could enhance the cost-efficiency of implementing large-scale nutrition monitoring surveys $^{(17)}$. The increased access to the Internet has fostered the development of many selfadministered dietary assessment methods, including web-based and smartphone-based tools $^{(18)}$. The overall quality of collected data from these tools is comparable with the interviewer-administered method ${ }^{(19)}$. Participants have greater flexibility and fewer time constraints to complete the survey ${ }^{(17)}$. Costs could be greatly reduced with automated coding and less interviewer involvement. Moreover, the incorporation of more objective food recognition features (e.g., photographs, barcodes) could enhance efficiency and reducing unintentional under-reporting in recording real-time food intake ${ }^{(20,21,22,23)}$. Review studies have indicated great potential for mobile dietary assessment applications to be used in largescale studies ${ }^{(20,24,25)}$. Hence, moving towards self-administered tools from intervieweradministered tools seems a promising effort to explore for future national food consumption surveys $^{(26)}$. However, the complexity of self-reporting tools is a real concern for certain people to participate and complete the survey ${ }^{(17)}$. A simplification of certain comprehensive features might be a crucial step in facilitating migrations from interviewer-administered tool to a self-administered tool.

The feature of recording mixed meal intake comprises complicated procedures in GloboDiet. Mixed recipes are collected through a specific recipe pathway(27), which starts by automatically searching entered recipes within a pre-existing standard recipe list ${ }^{(9,28)}$. The 
standard recipe is entered into the system unless the participants know that the actual recipe they consumed has different ingredient than the standard recipe. In this case, ingredients in standard recipes can be replaced, and the amounts of ingredients can be adjusted ${ }^{(15,29)}$. Different from portion size estimation of reported single food items which are always estimated "as consumed", for mixed recipes, more steps are needed to estimate the amount of each ingredient. After the portion size of the consumed mixed dish has been estimated, the ingredient amounts in the whole prepared recipe can be reported as raw or as consumed. With only raw amounts known, a consumed amount is calculated using pre-defined algorithms and standard food-specific coefficients (e.g., raw-to-cooked yield factors, density, or edible part coefficients) ${ }^{(9,10)}$. This additional ingredient adjustment is complicated to implement and requires much work and knowledge from the participants. Besides, estimating ingredient amounts in a mixed meal is without question a difficult task, given that people already find it hard to estimate portions in a single food item ${ }^{(14)}$. The common practice for current self-administered tools is to choose standard mixed dishes directly or to create new recipes from scratch ${ }^{(6,30)}$. Although omitting modifications to the standard recipes can save much effort, it could potentially bias the actual ingredient intake. Hence, the impact of using standard recipes without modifications on the nutrient and food group intake at the population level should be investigated.

This study aims to provide evidence to support the decision on whether a standard recipe modification feature in self-administered $24 \mathrm{hRs}$ or food diary apps is needed for large-scale dietary surveys. Firstly, we evaluated how often a home-prepared mixed meal is consumed in the Dutch diet and how often alterations were being made to standard recipes. Subsequently, we did a simulation analysis using national survey data in which standard recipes were adjusted by the interviewers and assessed the impact of ignoring these changes but using the standard ingredients. We then compared the observed food group and nutrient intake distributions of the population between the original and simulated data.

\section{Methods}

Data Collection

In this study, the importance of recipes in the Dutch diet was analysed and a simulation study was conducted using the data of the Dutch National Food Consumption Survey 2012-2016(31). This survey was conducted among 4313 Dutch men and women aged 1-79 years old. Subjects were excluded if they were pregnant, lactating, or institutionalized. Participants completed a questionnaire covering various background factors, such as educational level, working status, native country, family composition, various lifestyle factors, such as patterns of physical activity, smoking, use of alcoholic beverages and various general characteristics of the diet. Dietary intake of participants was collected through two $24 \mathrm{hRs}$ on non-consecutive days with 2-6 weeks in between. The $24 \mathrm{hRs}$ for children between 1 to 15 years old and older adults between 70 to 79 years old were collected by face-to-face interviews by trained dieticians 
with a food diary completed one day before the interview as an aid. For children aged 1 to 8 years, their parents or caretakers were interviewed. The $24 \mathrm{hRs}$ for 16 to 70 years olds were conducted through two telephone interviews. In both the face-to-face and the telephonebased $24 \mathrm{hR}$ interviews, a computer-assisted software called GloboDiet developed by the International Agency for Research on Cancer (IARC) was used ${ }^{(8)}$.

\section{Current Recipe Collection}

The feature within GloboDiet that could record mixed meal intakes was called the recipe pathway. As a starting point, a standard recipe list with 378 pre-defined recipes embedded in the recipe pathway was used if a pre-defined recipe resembled the mixed dish reported by the participants. Then, participants were asked whether the recipe was commercial or homemade. Commercial recipes were those with brand names from commercial sources such as supermarkets and restaurants. For home-prepared dishes, different procedures were followed depending on the participant's knowledge of their dishes. For those who were aware of the detailed information, an individual recipe was created by going through several steps to modify the standard ingredients according to their situations. For people not knowing much about their dishes, standard recipes were applied instead. For situations that ingredients were visually recognized in the mixed dish, ingredients in standard recipes were substituted, this type of recipes was regarded as a modified recipe. For ingredients that were reported as raw, raw-to-cooked yield factors and edible part coefficients were multiplied with the raw amount to calculate the consumed amount. A complete flow chart explaining the recipe pathway can be found in Figure 1. All reported food items, including the recipe ingredients, were linked to the most appropriate food code in the Dutch National Food Composition Database (NEVO table 2016/5.0) ${ }^{(32)}$ by trained dieticians. Each food item/ingredient were categorized according to the GloboDiet food group classification system ${ }^{(33)}$. 


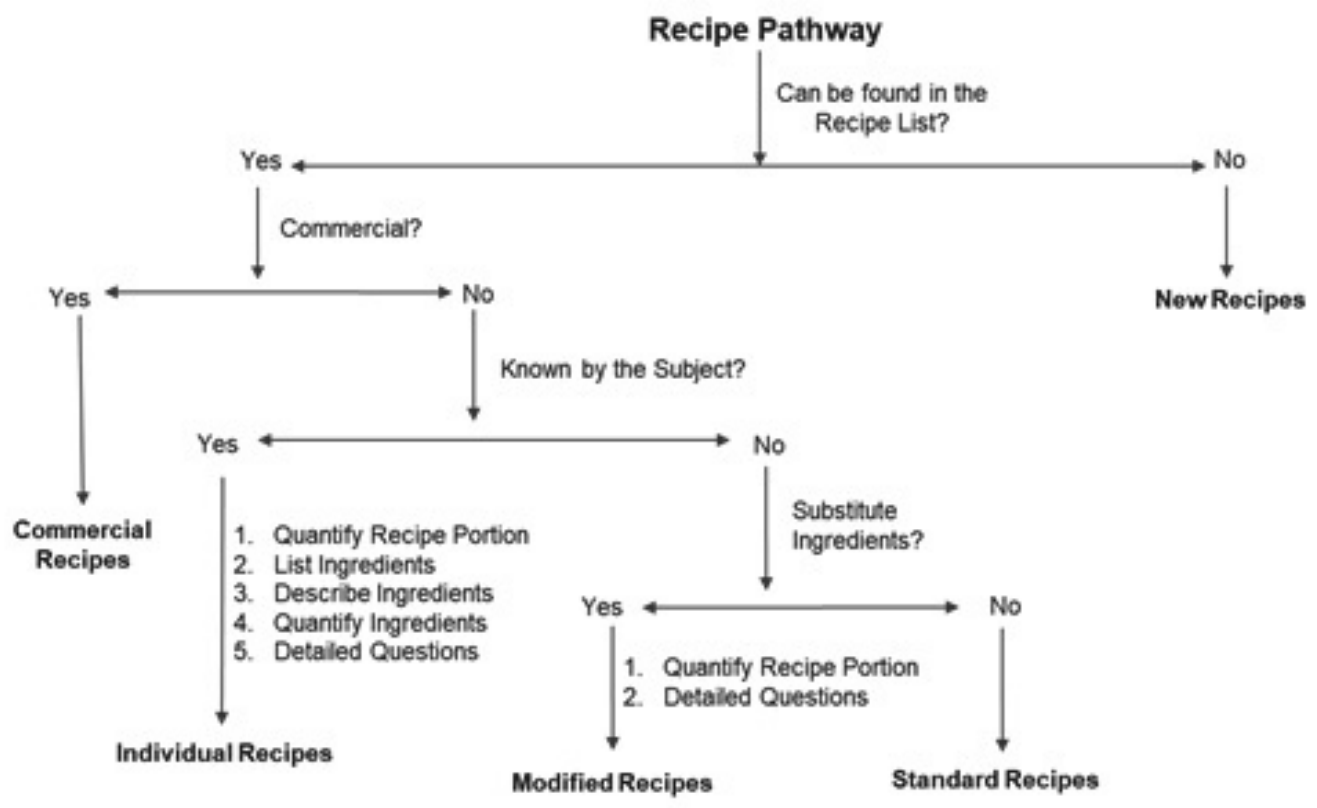

Figure 1. The flow chart of the mixed meal pathway in GloboDiet. Dishes were defined as homemade dishes if they could be found in the pre-defined recipe list and were not derived from commercial sources. Individual recipes were defined when people knew the information, they could substitute the predefined ingredients or adjust the amount of the ingredients of a standard recipe. For those who did not know the recipe, standard recipes would be used instead. For situations where the participants partly knew the recipe, adjustments of the ingredients were possible. These were regarded as modified recipes. New recipes were created if the name of the dish could not be found in the pre-defined recipe list.

Simulation Procedure

A two-scenario simulation study was conducted to evaluate whether the distributions of population nutrient and food group intake changed significantly when only standard recipes were used. The individual recipe scenario only ignored modifications to standard recipes for people who knew the recipes. In other words, the ingredients of individual recipes were switched to ingredients of standard recipes. The modified recipe scenario only ignored modifications to standard recipes during or after the interview for people who did not know all details of the recipe (but they could see some ingredients or had some insight in the used ingredients but not amounts). In both scenarios, the portion consumed for each recipe was kept the same with the original individual or modified recipe. The amount of ingredients were calculated according to the predefined percentage of the recipe total weight. All the ingredients were linked to the food code in the NEVO automatically if the same food item was linked already in the original database, otherwise they were linked by dieticians. The individual recipe scenario and the modified recipe scenario were also taken together in a combined scenario. Scenario analyses were run with all participants including those that did 
not use recipes, and in the subset of participants that did consume either mixed recipes that were reported as individual recipes or modified recipes. The details of preparing commercial recipes were not known by the participants, and newly created recipes were created from scratch without having a corresponding standard recipe to compare with. Hence, the ingredients were kept unchanged for recipes that were originally commercial, for unmodified standard recipes and for new recipes.

\section{Data Analysis}

The following study population characteristics were summarized. The highest educational level of the participants or the parents/carers of participants under the age of 19, who is the main earner of the family. Educational level was categorized into low (primary education, lower vocational education, advanced elementary education), middle (intermediate vocational education, higher secondary education), and high (higher vocational education and university). Percentages of energy and macronutrient intake consumed through recipes from the individual's total intake were calculated for the total population and per age and sex category. Percentage of energy intake consumed through recipes per eating occasion, recipe types, recipe groups was calculated. All population means were weighted for sociodemographic characteristics, day of the week and season of data collection, to give results that are representative for the Dutch population and representative for all days of the week and all seasons.

The nutrient level and quantities of food groups consumed were summarized per person by day and averaged over two days in both the dataset with original ingredients and the one with ingredients from standard recipes. The weighted mean, median, 5th, 25th, 75th, 95th percentile and the percentage differences of consumption per nutrient and food group between the original and the new dataset were calculated for the total population and within people who used individual and modified recipes in each scenario. The nutrient intake estimation was conducted for two scenarios, both separately and combined. The number of food items in each food group was also compared between the original state and the combined scenario. The descriptive summary and population nutrient intake distributions were conducted using the SAS 9.4, the replacement of ingredients from standard recipes to original dataset were conducted using R x64 3.5.0. The percentage differences between the original and newly linked dataset were calculated using Excel 2016 software.

\section{Results}

The general characteristics of the survey participants are shown in Table 1. The study included equal percentages for each age-gender group. The average BMIs for boys (18.0 $\left.\mathrm{kg} / \mathrm{m}^{2}\right)$ and males $\left(26.0 \mathrm{~kg} / \mathrm{m}^{2}\right)$ were similar with those for girls $\left(18.1 \mathrm{~kg} / \mathrm{m}^{2}\right)$ and females $\left(26.6 \mathrm{~kg} / \mathrm{m}^{2}\right)$, respectively. More than half of the boys and girls had a highly educated head of the household (54\%). More adult males (38\%) had a higher education level than females 
(28\%). The mean intake of energy per day was generally higher in boys (1988 kcal) and males $(2543 \mathrm{kcal})$ than in girls $(1685 \mathrm{kcal})$ and females $(1860 \mathrm{kcal})$. The percentages of energy consumed through mixed dishes were lower or equal to $10 \%$ for the four age-gender groups; adult female (10\%) consumed more energy through mixed dishes than other agegender groups.

Figure 2 illustrates the percentage of energy consumed through mixed dishes differentiated by eating occasions, by recipe types (new, individual, modified, standard) and by recipe groups based on the food group of the main ingredients. Dinner was the main occasion for consuming mixed dishes (73.2\%). More than half of the people who consumed mixed dishes knew the content of the recipe and reported individual recipes $(62.9 \%)$. The modified recipes $(15.1 \%)$ were reported as the second most frequent recipe type. Among all the recipe groups, energy from cereal- (52.5\%) and vegetable- (22.6) based mixed dishes were higher than other recipe groups.

Table 1. General characteristics of the population aged 1-79 years old from the Dutch National Food Consumption Survey 2012-2016, weighted for socio-demographic characteristics and season, and day of the week.

\begin{tabular}{|c|c|c|c|c|c|c|}
\hline \multirow{2}{*}{ Gender (n) } & & \multirow{2}{*}{$\begin{array}{l}\text { Total } \\
4313\end{array}$} & \multicolumn{2}{|c|}{ 1-18 years old } & \multicolumn{2}{|c|}{ 19-79 years old } \\
\hline & & & $\begin{array}{l}\text { Boys } \\
(1122)\end{array}$ & $\begin{array}{l}\text { Girls } \\
(1113)\end{array}$ & $\begin{array}{l}\text { Males } \\
(1043)\end{array}$ & $\begin{array}{l}\text { Females } \\
(1035)\end{array}$ \\
\hline \multirow{3}{*}{$\begin{array}{l}\text { Education } \\
\text { n(\%) }\end{array}$} & Low & $815(19)$ & \multirow{3}{*}{$\begin{array}{l}108(9) \\
413(37) \\
601(54)\end{array}$} & \multirow{3}{*}{$\begin{array}{l}105(9) \\
408(37) \\
600(54)\end{array}$} & \multirow{3}{*}{$\begin{array}{l}242(23) \\
406(39) \\
395(38)\end{array}$} & \multirow{3}{*}{$\begin{array}{l}360(35) \\
383(37) \\
292(28)\end{array}$} \\
\hline & Middle & $1628(38)$ & & & & \\
\hline & High & $1888(44)$ & & & & \\
\hline \multicolumn{3}{|c|}{ Mean BMI kg/m² (SD) } & $18.0(3.1)$ & $18.1(3.4)$ & $26.0(4.6)$ & $26.6(5.6)$ \\
\hline \multicolumn{3}{|c|}{$\begin{array}{l}\text { Mean Energy intake in kcal per day } \\
\text { (SD) }\end{array}$} & $1988(21)$ & $1685(16)$ & $2543(27)$ & $1860(19)$ \\
\hline \multicolumn{3}{|c|}{$\begin{array}{l}\text { Mean \% kcal from home-made recipes } \\
\text { (SD) }\end{array}$} & $8(0.32)$ & $8(0.34)$ & $9(0.38)$ & $10(0.53)$ \\
\hline
\end{tabular}

SD, standard deviation. 


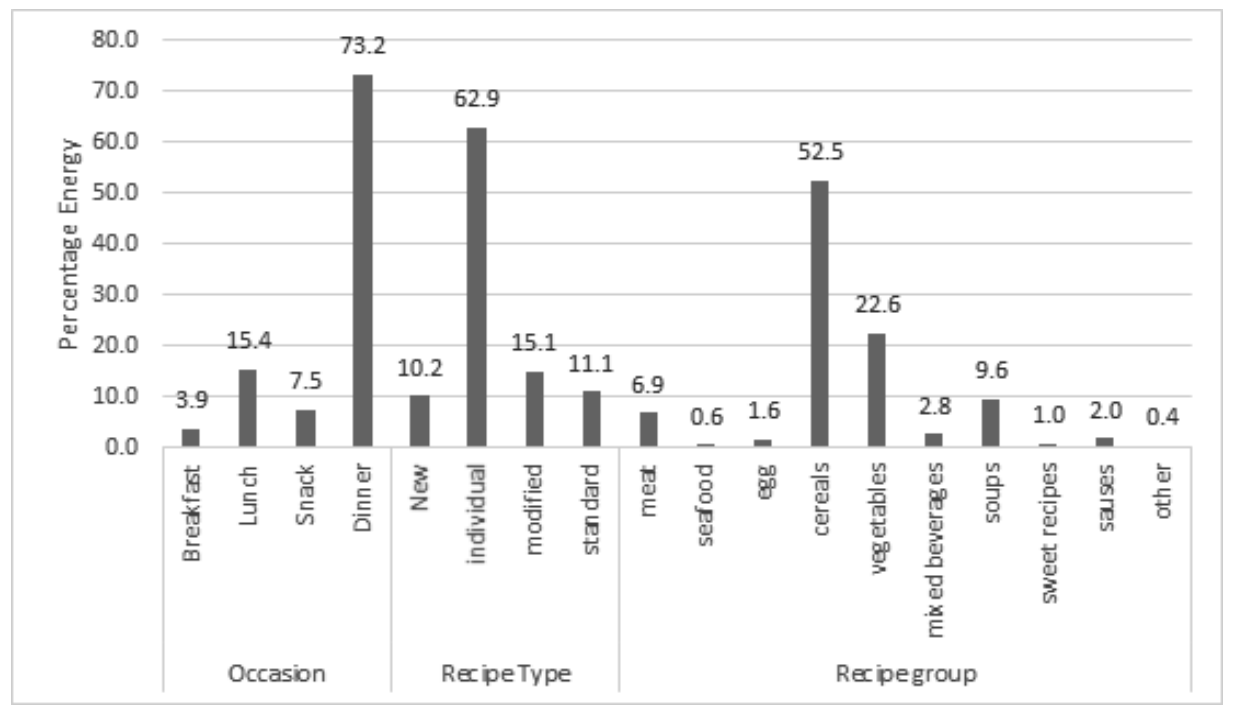

Figure 2. Energy consumed through mixed dishes partitioned (\%) by different occasions, recipe types and recipe groups from the Dutch National Food Consumption Survey 2012-2016.

Stratified by food groups, the impact of the combined scenario on the consumed amount of ingredients at a population level are shown in Table 2. In the individual recipe scenario, we disregarded modifications made by people who knew their recipes, while in the modified recipe scenario, the substitutions made by people who did not know the exact recipes were disregarded. Detailed results for sub-food groups can be found in Appendix 1. From Table 2 , the average of the percentage difference in mean intakes over all food groups was $-0.2 \%$, while the average of the absolute percentage difference was $1.6 \%$. For eight out of 17 food groups, the percentage difference in mean consumed amount was larger than $1 \%$ or lower than $-1 \%$ between the combined scenario and the original dataset. Among the food groups that were overestimated by the standard recipes, meat has the highest percentage difference (3.6\%). Specifically, ingredients from the meat group were overestimated the most by the standard recipes of hamburgers and meat wraps. Potatoes $(1.2 \%)$ and legumes $(0.7 \%)$ also showed an overestimation of the consumed amount but an underestimation in the count of the food ingredients by the standard recipes. Another observation was that the standard recipes tended to be less specific for certain food groups. For example, there were more unclassified meat products in standard recipes than in individual recipes (Appendix 1). A similar finding was also observed in the fats group.

For the food groups with an underestimated consumed amount by the standard recipes, soups and stocks had been underestimated to the greatest extent in average intake $(-6.6 \%)$. The underestimation was mainly due to the existence of water in standard recipes of soups that 
were made from soup powders, whereas stock from the soup group was reported in individual and modified recipes. Similarly, the total amount of vegetables was underestimated by the standard recipes, especially in spaghetti bolognese, greek salad, chicken-related dishes (e.g., wrap, curry, siam) and in different kinds of soups. On the contrary, there was a higher occurrence of different vegetables in standard ingredients. When we looked at the detailed results of food subgroups (Appendix 1), fruiting vegetables, cabbages, mushrooms, and stalk vegetables were the main contributors to the contradictory result. In other words, these subgroups were used more often in standard recipes but in small amounts.

As for the results of the nutrient analysis, Table 3 shows the percentage difference and the difference of the actual amount of 26 nutrients between the combined scenario and the original dataset within the total population. The average of the percentage difference was $0.6 \%$ for the absolute mean intakes across all nutrients. The averages for the other five percentiles of the intake distributions were slightly higher; the $25^{\text {th }}$ percentile has the highest average of $1.0 \%$. The percentage difference in mean of five nutrients was larger than $1.0 \%$ or lower than $-1.0 \%$. Most nutrient intakes $(73 \%)$ were underestimated by using standard recipes, with an average percentage difference of $-0.4 \%$ for the population mean intakes. The largest negative mean percentage difference was in DHA (-2.3\%) with an actual amount difference of $-2.6 \mathrm{mg}$, while the largest positive mean percentage difference was in vitamin B1 $(1.8 \%)$ with an actual amount difference of $0.02 \mathrm{mg}$. A relatively larger percentage difference with a low actual amount difference was also observed in trans fatty acids $(-1.1 \%,-0.01 \mathrm{~g})$. To compare the impact to the total population with only those who consumed mixed dishes, seven nutrients that have higher percentage differences than the other 19 nutrients from the combined scenario are included in Figure 3a. The impact within people who consumed mixed dishes was larger than the impact on the total population for every nutrient. When we looked at Appendix 2b that has the percentage, and actual amount difference for all nutrients, the effect within people consumed mixed dishes has more nutrients with a mean percentage difference larger than $1.0 \%$ or lower than $-1.0 \%$ than within total population.

The separate effects of each scenario on the nutrient intake of the total population is shown in Figure 3b. Either scenario has a smaller impact than the combined effect as shown in Figure 3a. The individual recipe scenario has a larger impact on the nutrient intake distribution than the modified recipe scenario. The results with all nutrients for each scenario separately is shown in Appendix $3 a$ \& $3 b$. The individual recipe scenario has an average of the absolute mean percentage difference of $0.5 \%$ with five nutrients larger than $1.0 \%$ or lower than $-1.0 \%$. While the modified recipe scenario has an average of the absolute mean percentage difference of $0.2 \%$ with all nutrients fell within $-1.0 \%$ to $1.0 \%$. About $63 \%$ of the nutrients were underestimated in scenario 1 , while $88 \%$ of the nutrients were underestimated in scenario 2. Figure $3 \mathrm{a}$ and $3 \mathrm{~b}$ also illustrate that the intake of most nutrients was underestimated by using standard recipes. Exceptions were vitamin B1 and ALA. 
Vitamin B1 was overestimated in all scenarios. ALA showed contradictory results between the two scenarios and was higher in combined scenarios than the original dataset.

Table 2. The percentage and amount difference of the food group intake distribution of the population between the combined scenario and the original data.

\begin{tabular}{|c|c|c|c|c|c|c|c|c|c|}
\hline \multirow[b]{2}{*}{ Food Groups } & \multicolumn{4}{|c|}{ Percentage Difference (\%) } & \multicolumn{4}{|c|}{ Amount Difference (g) } & \multirow{3}{*}{$\begin{array}{c}\text { Difference } \\
\text { in the } \\
\text { number of } \\
\text { ingredient } \\
\text { occurrence } \\
-31\end{array}$} \\
\hline & Mean & Median & P75 & P95 & Mean & Median & P75 & P95 & \\
\hline Potatoes and other tubers & 1.2 & 2.5 & 0.0 & 0.9 & 0.8 & 1.5 & 0.0 & 1.9 & \\
\hline Vegetables & -4.0 & -6.4 & -4.1 & -3.8 & -5.3 & -7.2 & -7.3 & -12.0 & 1454 \\
\hline Legumes & 0.7 & 0.0 & 0.0 & 0.0 & 0.0 & 0.0 & 0.0 & 0.0 & -18 \\
\hline Fruits, nuts and seeds, olives & -0.6 & -0.8 & -1.1 & 0.0 & -0.7 & -0.8 & -2.1 & -0.1 & 50 \\
\hline Dairy products and substitutes & 0.1 & 0.6 & 0.0 & 0.4 & 0.4 & 1.9 & 0.0 & 3.4 & 254 \\
\hline Cereals and cereal products & 1.6 & 1.4 & 1.4 & 2.3 & 3.1 & 2.5 & 3.5 & 8.5 & 163 \\
\hline Meat, meat products and substitutes & 3.6 & 3.8 & 2.8 & 1.7 & 3.5 & 3.3 & 3.7 & 3.9 & 49 \\
\hline Fish, shellfish and amphibians & -3.0 & 0.0 & 0.0 & 0.0 & -0.5 & 0.0 & 0.0 & 0.0 & -42 \\
\hline Eggs and egg products & 2.6 & 0.0 & 0.0 & 6.2 & 0.3 & 0.0 & 0.0 & 3.1 & 88 \\
\hline Fats and oils & 2.4 & 3.1 & 1.9 & -0.2 & 0.5 & 0.6 & 0.6 & -0.1 & 662 \\
\hline Sugar and confectionery & -0.1 & 0.1 & 0.0 & 0.0 & 0.0 & 0.0 & 0.0 & 0.0 & -68 \\
\hline Cakes and sweet biscuits & 0.0 & 0.0 & 0.0 & 0.0 & 0.0 & 0.0 & 0.0 & 0.0 & -4 \\
\hline Non-alcoholic beverages & 0.2 & 0.2 & 0.0 & -0.5 & 3.6 & 2.9 & 0.5 & -14.9 & 416 \\
\hline Condiments, spices, sauces and yeast & -0.5 & -1.7 & 0.0 & 0.2 & -0.2 & -0.4 & 0.0 & 0.2 & 32 \\
\hline Soups and stocks & -6.6 & 0.0 & -10.9 & -4.3 & -2.8 & 0.0 & -6.8 & -9.9 & -460 \\
\hline Miscellaneous & -0.1 & 0.0 & 0.0 & 0.0 & 0.0 & 0.0 & 0.0 & 0.0 & -9 \\
\hline Savoury snacks & -0.1 & 0.0 & 0.0 & 0.0 & 0.0 & 0.0 & 0.0 & 0.0 & -11 \\
\hline Average ( |Percentage Difference|) & 1.6 & 1.2 & 1.3 & 1.2 & - & - & - & - & - \\
\hline Average ( Percentage Difference ) & -0.2 & 0.2 & -0.6 & 0.2 & - & - & - & - & - \\
\hline
\end{tabular}

P75, $75^{\text {th }}$ percentile. P90, 95 ${ }^{\text {th }}$ percentile. | Percentage Difference $\mid:$ the absolute value of percentage difference. 
Table 3. The percentage and amount difference of the nutrient intake distribution of the population between the combined scenario and the original data.

\begin{tabular}{|c|c|c|c|c|c|c|c|c|c|}
\hline \multirow[b]{2}{*}{ Nutrients } & \multicolumn{6}{|c|}{ Percentage Difference (\%) } & \multicolumn{3}{|c|}{ Amount Difference } \\
\hline & Mean & P5 & P25 & Median & P75 & P95 & Mean & P5 & P95 \\
\hline Energy (kcal) & 0.2 & 1.4 & 0.5 & -0.3 & -0.4 & -0.1 & 4 & 16 & -4 \\
\hline Protein (g) & 0.0 & 0.1 & 0.3 & 0.2 & 0.6 & 1.4 & 0.0 & 0.0 & 1.8 \\
\hline Carbohydrates (g) & 0.6 & 0.6 & 0.4 & 0.7 & 0.3 & 0.6 & 1.3 & 0.7 & 2.4 \\
\hline Mono- and disaccharides (g) & -0.1 & 0.1 & -0.1 & -0.2 & -0.2 & -0.3 & -0.1 & 0.0 & -0.6 \\
\hline Fibre (g) & -0.8 & -0.2 & -1.2 & -0.6 & -0.5 & 0.1 & -0.2 & 0.0 & 0.0 \\
\hline Fat (g) & -0.2 & 0.8 & 0.0 & -0.4 & -1.1 & 0.0 & -0.1 & 0.3 & 0.0 \\
\hline SFA (g) & -0.5 & -0.4 & 0.5 & -0.7 & 0.2 & -0.8 & -0.1 & 0.0 & -0.4 \\
\hline ALA (g) & 0.2 & 4.2 & 1.0 & -0.1 & 1.0 & -1.6 & 0.00 & 0.02 & -0.06 \\
\hline TFA (g) & -1.1 & -2.5 & -0.6 & -1.2 & -1.2 & 0.3 & 0.0 & 0.0 & 0.0 \\
\hline DHA (mg) & -2.3 & 0.0 & -9.4 & -10.1 & -2.6 & -2.1 & -2.63 & 0.00 & -14.51 \\
\hline Calcium (mg) & -0.1 & 1.1 & 0.5 & 0.0 & 0.3 & -1.3 & -1 & 4 & -23 \\
\hline Iron (mg) & -0.8 & 0.4 & -0.6 & -0.9 & -1.1 & -0.5 & -0.1 & 0.0 & -0.1 \\
\hline Sodium (mg) & 0.4 & -1.2 & 0.3 & -0.1 & -0.6 & 1.3 & 9 & -13 & 54 \\
\hline Potassium (mg) & -0.5 & 0.3 & 0.0 & -1.1 & 0.0 & 0.2 & -16 & 4 & 8 \\
\hline Zinc (mg) & -0.2 & -0.3 & -0.2 & 0.1 & -1.0 & -1.2 & -0.02 & -0.01 & -0.21 \\
\hline Beta-carotene $(\mu \mathrm{g})$ & -1.3 & 2.4 & -0.3 & 0.0 & 1.6 & -2.8 & -27 & 5 & -207 \\
\hline Retinol $(\mu \mathrm{g})$ & 0.2 & 2.1 & 0.5 & 0.1 & 0.3 & -0.2 & 1 & 3 & -4 \\
\hline Folate equivalents $(\mu \mathrm{g})$ & -0.9 & -0.1 & -1.0 & -1.2 & -1.0 & -0.2 & -2.1 & -0.1 & -0.8 \\
\hline Vitamin B1 (mg) & 1.8 & 1.0 & 2.1 & 0.8 & 1.7 & 3.6 & 0.02 & 0.00 & 0.07 \\
\hline Vitamin B2 (mg) & -0.2 & 0.6 & -1.1 & 0.0 & -0.3 & -0.6 & 0.00 & 0.00 & -0.02 \\
\hline Vitamin B3 (mg) & -0.4 & -0.8 & -0.9 & -1.3 & -1.2 & 0.2 & -0.1 & -0.1 & 0.1 \\
\hline Vitamin B6 (mg) & -0.5 & -0.2 & -0.3 & -1.4 & -0.5 & 0.7 & -0.008 & -0.002 & 0.021 \\
\hline Vitamin B12 $(\mu \mathrm{g})$ & -0.5 & -1.9 & -0.5 & -0.4 & -1.0 & 0.0 & -0.02 & -0.03 & 0.00 \\
\hline Vitamin C (mg) & -1.8 & -0.1 & -1.8 & -1.8 & -1.8 & -2.6 & -2 & 0 & -5 \\
\hline Vitamin D $(\mu \mathrm{g})$ & -0.2 & 0.0 & 0.1 & 0.0 & -0.4 & 0.6 & 0.0 & 0.0 & 0.0 \\
\hline Vitamin E $(\mu \mathrm{g})$ & -0.6 & -1.1 & -0.6 & -0.7 & -0.6 & -0.1 & -0.1 & -0.1 & 0.0 \\
\hline Average ( $\mid$ Percentage Difference $\mid$ ) & 0.6 & 0.9 & 1.0 & 0.9 & 0.8 & 0.9 & - & - & - \\
\hline Average ( Percentage Difference ) & -0.4 & 0.2 & -0.5 & -0.8 & -0.4 & -0.2 & - & - & - \\
\hline
\end{tabular}

P5, 5th percentile. $\mathrm{P} 25,25$ th percentile. $\mathrm{P} 75,75$ th percentile. P90, 95th percentile. SFA, saturated fatty acids. ALA, alpha-Linolenic acids. TFA, trans-fatty acids. DHA, docosahexaenoic acids. | Percentage Difference |: the absolute value of percentage difference. 


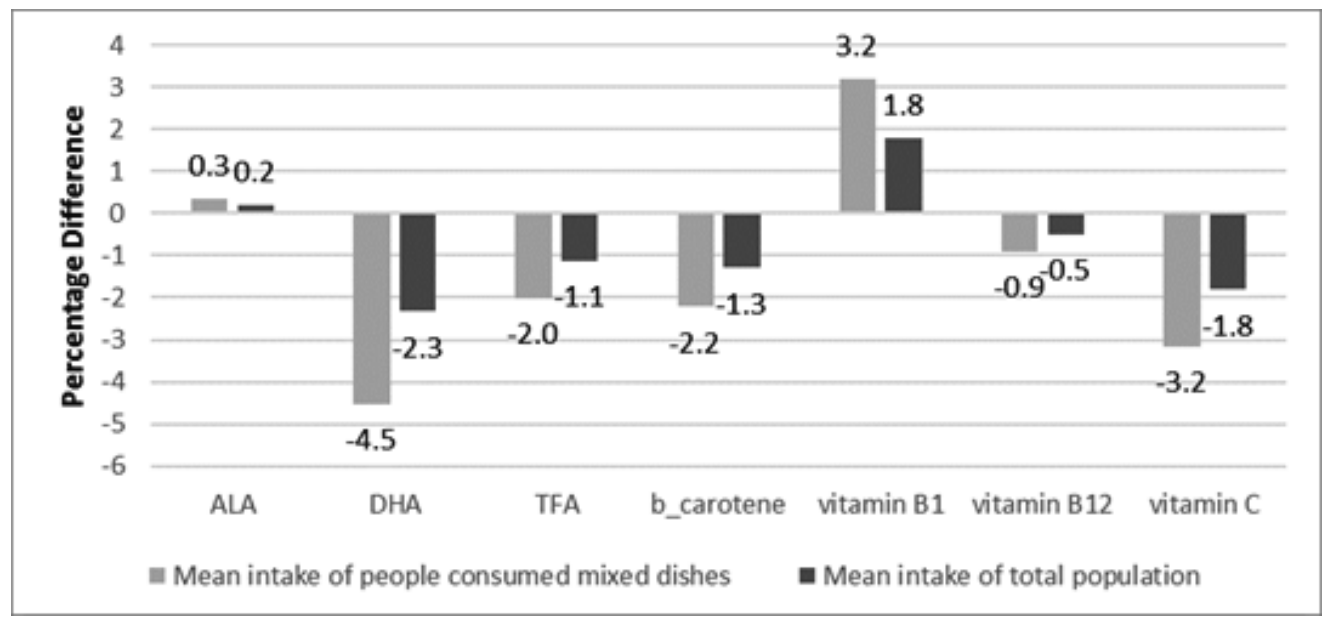

Figure 3a. The percentage difference of the mean intake of 7 nutrients of the total population and within people who consumed mixed dishes between the combined scenario and the original dataset.

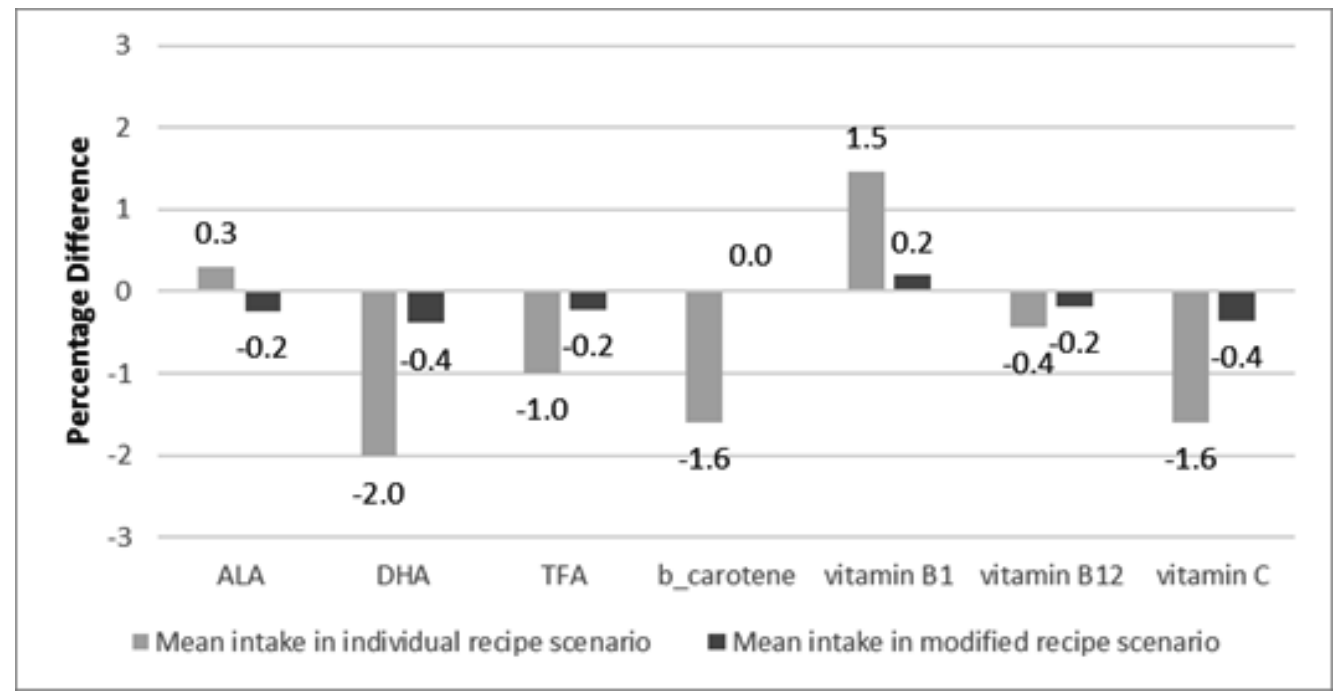

Figure $3 \mathrm{~b}$. The percentage difference of the mean intake of 7 nutrients of the total population between each scenario and the original dataset. 


\section{Discussion}

A replacement of complete recipe recording steps with a simplified recipe recording procedure would help improve the cost-effectiveness of recording mixed meal intake and was explored to be used in the Dutch National Food Consumption Surveys (DNFCS). Therefore the impact of replacing individual with standard recipes was investigated using data collected in DNFCS 2012-2016. With a few exceptions, this study found that using only pre-defined standard recipes caused less than one percent differences in mean nutrient intakes and food consumption compared to standard recipes being modified according to participant declaration. The main contributing factor for the insignificant impact was the small portion of the energy consumed (approximately 10\%) from home-made mixed meals, according to DNFCS 2012-2016. This observation is in line with the trend of preparing less mixed dishes at home due to peoples' tendency to eating quick and ready meals ${ }^{(34)}$. Also, compared to countries where mixed dishes were dominant ${ }^{(35)}$, the western diet includes relatively few dishes that mix all ingredients ${ }^{(36)}$. An additional explaining factor was that $20 \%$ of the homemade mixed meals were entered as new recipes or unmodified standard recipes, both of which could not be simplified in this study.

Despite the small overall difference in main food groups, a larger difference was found in some subgroups of the main food group. The reason is that the standard recipes contained more ingredients from undefined food subgroups while individual recipes contained more ingredients from specific food subgroups. A seemingly contradictory outcome was found in several food groups where the average consumed amount was lower, while the number of food items was higher in standard recipes, the vegetable group is a notable example of this. One possible explanation might be that the participants deemed vegetables as healthy foods hence overestimated the consumed amount in individual recipes ${ }^{\left({ }^{37}\right)}$. Another reason is that the standard recipes in our study were purposely created with more varieties of vegetables in smaller portion size of each type in order to make them representative for different versions of a recipe (lasagne with mushrooms, or with leek, or with carrots).

The change in the ingredients would inevitably cause a change in nutrient intake ${ }^{(38,39)}$. The overall difference was small across nutrients with only a few exceptions. DHA has the largest average percentage difference and was underestimated when replacing individual recipes with standard recipes $(-2.3 \%)$, which was mainly due to the fact that people put fish in dishes that do not have fish in the corresponding standard recipes (e.g., oven dishes, salads, foreign dishes). On the contrary, vitamin $\mathrm{B} 1$ has the largest positive average percentage difference of $1.8 \%$, which was probably due to the higher average amount of dairy, cereals, and meat in standard recipes. These differences seem unsubstantial for dietary monitoring purposes with a large sample size. However, to better accommodate real-life variations, the development of future standard recipes should consider the fact that people tend to take fewer varieties from certain food groups (e.g., vegetables) but higher amounts of available varieties 
in certain dishes. The specificity of food subgroups should be defined in standard recipes with ingredients from, for example, the meat group. Also, acknowledge that people might exclude or replace the main ingredients of certain dishes with ingredients from other food groups. Without the modification functionality, identical standard recipes with different main ingredient options should be listed individually, with key ingredients shown in the recipe title for easier identification. A study comparing nutrition results from more varieties of unmodifiable standard recipes with results from original modifiable standard recipes could provide more relevant insight.

As far as we know, this is the first study investigating the impact of replacing individual with standard recipes. The study contained a large sample size $(n=4313)$, the population was representative of the Dutch population, and the survey results were representative for all days of the week and all seasons. The study results are transferable to surveys which use Globodiet as their main instrument of collecting dietary data; however, it may not apply to countries where mixed dishes are dominant in the diet. Unlike many other large food consumption surveys that allocate a composite dish into one food group ${ }^{(40,41)}$, surveys that use Globodiet disaggregate ingredients of recipes and distinguish the food group of every ingredient ${ }^{(42)}$. The disaggregation simplifies the procedure of replacing old ingredients with standard ingredients and calculating nutrient and food group difference between the original and new scenarios. Another advantage of the study is that the between-person variation did not impact the results since the manipulated dataset was derived from the original dataset, and thus on data from the same participants ${ }^{(37)}$.

There are also some limitations to the study. Firstly, some of the complex foods were not considered as recipes in Globodiet ${ }^{(9)}$, such as cakes, biscuits, desserts, sauces, and some snacks. As a result, the percentage of the home-prepared mixed meal might have been underestimated as well as the impact on intake. However, the influence is estimated to be small due to a high proportion of eating industrially prepared food and out-of-home eating for sweets, especially for northern European countries such as the Netherlands ${ }^{(39,43,44)}$. Secondly, only the impact on food groups and nutrients were considered, while other aspects related to food can also be important. For example, since standard recipes contain mostly generic food items, this would underestimate the consumption of branded or specific food items, and hence their environmental impact as well as exposure to potentially harmful substances of the population. Lastly, the quality, completeness, and specificity of the standard recipe database is also an essential aspect in estimating the actual intake of the population. In our study, the standard recipe list was derived from a widely-used cookbook in the Netherlands, the deviation of standard recipes from the real-life intake is unknown.

As opposed to creating a new individual recipe from scratch, good quality standard recipes could save time, supplement commonly forgotten ingredients such as seasonings ${ }^{(7,35)}$, and 
correct misreporting out of embarrassment and inconvenience ${ }^{(45)}$. Hence, standard recipes were embedded in most of the dietary apps and software, as well as dietary assessment surveys in many countries ${ }^{(39,46)}$. While numerous commercial and research-based apps have the option of creating new individual recipes ${ }^{(47)}$, there are no self-administered methods incorporated modifiable standard recipes as far as we know ${ }^{(48)}$. The reason for the less popularity of modifiable standard recipes in self-administered software is that incorporating recipe modification would increase the time and effort for the participants and part of the respondents might not provide valuable answers due to their limited knowledge about the recipe. Also, when applying technologies like photo recognition and analysis in smartphones ${ }^{(45,49,50)}$, challenges exist especially for mixed dishes where not all ingredients are visible ${ }^{(51)}$.

According to the study results and current limitation on technology, a recipe function that could balance the workload of participants and capture deviation with real-life intakes is proposed. In self-reported food diaries or $24 \mathrm{hRs}$, participants could choose well-described unchangeable standard recipes if they are representative for the real preparation habits of the population. For participants that have consumed a mixed dish that cannot be classified as one of the available recipes, an individual recipe could be created. In this way, the number of participants that are requested to provide recipe details is limited. Such an approach needs to be evaluated in terms of usability for the users, and in terms of the validity of the consumption data.

Conclusion: Disregarding modification steps of a recipe functionality in $24 \mathrm{hR}$ software has a small impact on the distribution of food group consumption and nutrient intake of the Dutch population. Therefore, there seems to be minor loss in validity for food group and nutrient intake if no recipe function is available and mixed dishes are treated as food (with standard ingredients). Using good quality standard recipes without modification is a promising solution for reducing participant burden on self-administered $24 \mathrm{hR}$ or food diary. 


\section{References:}

1. Arens-Volland AG, Spassova L, Bohn T (2015) Promising approaches of computersupported dietary assessment and management-Current research status and available applications. Int J Med Inform 84, 997-1008.

2. Mozaffarian D, Wilson PWF, Kannel WB (2008) Beyond established and novel risk factors - Lifestyle risk factors for cardiovascular disease. Circulation 117, 3031-3038.

3. Huybrechts I, Aglago EK, Mullee A et al. (2017) Global comparison of national individual food consumption surveys as a basis for health research and integration in national health surveillance programmes. Proc Nutr Soc 76, 549-567.

4. Rippin H, Hutchinson J, Evans C et al. (2018) National nutrition surveys in Europe: a review on the current status in the 53 countries of the WHO European region. Food Nutr Res 62.

5. Ahluwalia N, Dwyer J, Terry A et al. (2016) Update on NHANES Dietary Data: Focus on Collection, Release, Analytical Considerations, and Uses to Inform Public Policy. Adv Nutr 7, 121-134.

6. Jacques S, Lemieux S, Lamarche B et al. (2016) Development of a Web-Based 24-h Dietary Recall for a French-Canadian Population. Nutrients 8.

7. Shim J-S, Oh K, Kim HC (2014) Dietary assessment methods in epidemiologic studies. Epidemiol Health 36, e2014009-e2014009.

8. Slimani N, Casagrande C, Nicolas G et al. (2011) The standardized computerized 24-h dietary recall method EPIC-Soft adapted for pan-European dietary monitoring. Eur J Clin Nutr 65 Suppl 1, S5-15.

9. Slimani N, Deharveng G, Charrondiere RU et al. (1999) Structure of the standardized computerized 24-h diet recall interview used as reference method in the 22 centers participating in the EPIC project. European Prospective Investigation into Cancer and Nutrition. Comput Methods Programs Biomed 58, 251-266.

10. EFSA (2014) Guidance on the EU Menu methodology. In EFSA Journal, vol. 12, pp. 3944: European Food Safety Authority.

11. Brussaard JH, Lowik MR, Steingrimsdottir L et al. (2002) A European food consumption survey method--conclusions and recommendations. Eur J Clin Nutr 56 Suppl 2, S89-94.

12. de Boer EJ, Slimani N, van 't Veer P et al. (2011) The European Food Consumption Validation Project: conclusions and recommendations. Eur J Clin Nutr 65, S102-S107.

13. Ocke M, Brants H, Dofkova M et al. (2015) Feasibility of dietary assessment methods, other tools and procedures for a pan-European food consumption survey among infants, toddlers and children. Eur J Nutr 54, 721-732.

14. Ambrus Á, Horváth Z, Farkas Z et al. (2013) Pilot study in the view of a Pan-European dietary survey - adolescents, adults and elderly. EFSA Supporting Publications 10, 508E-n/a.

15. Thompson FE, Subar AF (2017) Dietary assessment methodology. In Nutrition in the Prevention and Treatment of Disease, pp. 5-48: Elsevier. 
16. Ocké MC, Slimani N, Brants H et al. (2011) Potential and requirements for a standardized pan-European food consumption survey using the EPIC-Soft software. Eur J Clin Nutr 65, S48.

17. Touvier M, Kesse-Guyot E, Mejean C et al. (2011) Comparison between an interactive web-based self-administered $24 \mathrm{~h}$ dietary record and an interview by a dietitian for large-scale epidemiological studies. Br J Nutr 105, 1055-1064.

18. Taren D, Dwyer J, Freedman L et al. (2002) Dietary assessment methods: where do we go from here? Public Health Nutr 5.

19. Kirkpatrick SI, Subar AF, Douglass D et al. (2014) Performance of the Automated Self-Administered 24-hour Recall relative to a measure of true intakes and to an interviewer-administered 24-h recall. Am J Clin Nutr 100.

20. Ambrosini GL, Hurworth M, Giglia R et al. (2018) Feasibility of a commercial smartphone application for dietary assessment in epidemiological research and comparison with 24-h dietary recalls. Nutr J 17, 5.

21. Illner AK, Freisling H, Boeing $H$ et al. (2012) Review and evaluation of innovative technologies for measuring diet in nutritional epidemiology. Int J Epidemiol 41, 1187 1203.

22. Maringer M, van't Veer P, Klepacz N et al. (2018) User-documented food consumption data from publicly available apps: an analysis of opportunities and challenges for nutrition research. Nutr J 17, 59.

23. Cade JE (2017) Measuring Diet in the 21st Century: Use of New Technologies. Proc Nutr Soc 76, 276-282.

24. Eldridge AL, Piernas C, Illner AK et al. (2018) Evaluation of New Technology-Based Tools for Dietary Intake Assessment-An ILSI Europe Dietary Intake and Exposure Task Force Evaluation. Nutrients 11.

25. Sharp DB, Allman-Farinelli M (2014) Feasibility and validity of mobile phones to assess dietary intake. Nutrition 30, 1257-1266.

26. Subar AF, Kirkpatrick SI, Mittl B et al. (2012) The Automated Self-Administered 24hour dietary recall (ASA24): a resource for researchers, clinicians, and educators from the National Cancer Institute. J Acad Nutr Diet 112, 1134-1137.

27. Cosgrove M, Flynn A, Kiely M (2005) Impact of disaggregation of composite foods on estimates of intakes of meat and meat products in Irish adults. Public Health Nutr 8, 327-337.

28. Raper N, Perloff B, Ingwersen L et al. (2004) An overview of USDA's Dietary Intake Data System. J Food Compos Anal 17, 545-555.

29. Slimani N, Ferrari P, Ocke M et al. (2000) Standardization of the 24-hour diet recall calibration method used in the european prospective investigation into cancer and nutrition (EPIC): general concepts and preliminary results. Eur J Clin Nutr 54, 900917.

30. Leclercq C, Arcella D, Piccinelli R et al. (2009) The Italian National Food Consumption Survey INRAN-SCAI 2005-06: main results in terms of food consumption. Public Health Nutr 12, 2504-2532. 
31. Van Rossum CT, Nelis K, Wilson C et al. (2018) National dietary survey in 2012-2016 on the general population aged 1-79 years in the Netherlands. EFSA Supporting Publications 15.

32. RIVM (2011) NEVO-table. Series NEVO-table. https://nevo-online.rivm.nl/ (accessed July 2018).

33. Van Rossum C, Buurma-Rethans E, Vennemann F et al. (2016) The diet of the Dutch: Results of the first two years of the Dutch National Food Consumption Survey 20122016.

34. Geurts M, Bakel AMv, Van Rossum CT et al. (2016) Food consumption in the Netherlands and its determinants. Bilthoven, The Netherlands: National Institute for Public Health and the Environment.

35. Yun SH, Choi B-Y, Kim M-K (2009) The effect of seasoning on the distribution of nutrient intakes by a food-frequency questionnaire in a rural area. Korean Journal of Nutrition 42, 246-255.

36. Wang O, Gellynck X, Verbeke W (2016) Perceptions of Chinese traditional food and European food among Chinese consumers. British Food Journal 118, 2855-2872.

37. Macdiarmid J, Blundell J (1998) Assessing dietary intake: Who, what and why of under-reporting. Nutr Res Rev 11, 231-253.

38. Fitt E, Mak TN, Stephen AM et al. (2010) Disaggregating composite food codes in the UK National Diet and Nutrition Survey food composition databank. Eur J Clin Nutr 64, S32.

39. Fitt E, Prynne CJ, Teucher B et al. (2009) National Diet and Nutrition Survey: Assigning mixed dishes to food groups in the nutrient databank. J Food Compos Anal 22, S52-S56.

40. Sui Z, Raubenheimer D, Rangan A (2017) Consumption patterns of meat, poultry, and fish after disaggregation of mixed dishes: secondary analysis of the Australian National Nutrition and Physical Activity Survey 2011-12. BMC Nutrition 3, 52.

41. Agudo A (2005) Measuring intake of fruit and vegetables. Kobe, Japan: World Health Organization.

42. Roe M, Bell S, Oseredczuk M et al. (2013) Updated food composition database for nutrient intake. EFSA Supporting Publications 10, 355E.

43. Slimani N, Deharveng G, Southgate DA et al. (2009) Contribution of highly industrially processed foods to the nutrient intakes and patterns of middle-aged populations in the European Prospective Investigation into Cancer and Nutrition study. Eur J Clin Nutr 63 Suppl 4, S206-225.

44. Orfanos P, Naska A, Trichopoulos D et al. (2007) Eating out of home and its correlates in 10 European countries. The European Prospective Investigation into Cancer and Nutrition (EPIC) study. Public Health Nutr 10, 1515-1525.

45. Macdiarmid JI, Blundell JE (1997) Dietary under-reporting: what people say about recording their food intake. Eur J Clin Nutr 51, 199-200. 
46. Shinozaki N, Murakami K, Masayasu S et al. (2019) Development and simulated validation of a dish composition database for estimating food group and nutrient intakes in Japan. Public Health Nutr, 1-14.

47. Zhang L, Nawijn E, Boshuizen H et al. (2019) Evaluation of the Recipe Function in Popular Dietary Smartphone Applications, with Emphasize on Features Relevant for Nutrition Assessment in Large-Scale Studies. Nutrients 11.

48. Institute TNC (2018) Instructions for the ASA24 Researcher Website: ASA24-2018 (U.S. version only), The National Cancer Institute.

49. Bucher T, Weltert M, Rollo ME et al. (2017) The international food unit: a new measurement aid that can improve portion size estimation. Int J Behav Nutr Phys Act $14,124$.

50. Yang Y, Jia W, Bucher T et al. (2019) Image-based food portion size estimation using a smartphone without a fiducial marker. Public Health Nutr 22, 1180-1192.

51. Naska A, Lagiou A, Lagiou P (2017) Dietary assessment methods in epidemiological research: current state of the art and future prospects. F1000Res 6, 926. 


\section{Appendix 1: Stratified by food sub-groups, the impact of the combined} scenario on the consumed amount of ingredients.

\begin{tabular}{|c|c|c|c|c|c|c|}
\hline \multirow[b]{2}{*}{ Food Groups } & \multirow[b]{2}{*}{ Food sub-groups } & \multicolumn{2}{|c|}{$\begin{array}{l}\text { Percentage } \\
\text { Difference }\end{array}$} & \multicolumn{2}{|c|}{$\begin{array}{l}\text { Amount } \\
\text { Difference }\end{array}$} & \multirow[b]{2}{*}{ Occurrence } \\
\hline & & Mean & P95 & Mean & P95 & \\
\hline \multirow[t]{2}{*}{$\begin{array}{l}\text { Potatoes and other } \\
\text { tubers }\end{array}$} & $\begin{array}{l}\text { Unclassified, mixed and other } \\
\text { tubers }\end{array}$ & -24.2 & 0.0 & -0.1 & 0.0 & -13 \\
\hline & Potatoes & 1.3 & 0.9 & 1.0 & 1.9 & -18 \\
\hline \multirow[t]{9}{*}{ Vegetables } & $\begin{array}{l}\text { Unclassified, mixed } \\
\text { salad/vegetables }\end{array}$ & -18.4 & -13.7 & -2.2 & -11.0 & -266 \\
\hline & Leafy vegetables & -2.9 & -2.5 & -0.6 & -2.4 & -169 \\
\hline & Fruiting vegetables & -5.7 & -1.9 & -2.8 & -3.2 & 71 \\
\hline & Root vegetables & 4.7 & -1.9 & 0.6 & -1.6 & 635 \\
\hline & Cabbages & -2.5 & -4.7 & -0.5 & -5.3 & 209 \\
\hline & Mushrooms & -12.3 & -14.5 & -0.4 & -2.8 & 137 \\
\hline & Grain and pod vegetables & 1.1 & 0.8 & 0.0 & 0.1 & -21 \\
\hline & Leek, onion, garlic & 5.6 & -1.5 & 0.7 & -0.7 & 668 \\
\hline & Stalk vegetables, sprouts & -7.3 & -26.3 & -0.2 & -2.2 & 190 \\
\hline Legumes & Legumes & 0.7 & 0.0 & 0.0 & 0.0 & -18 \\
\hline \multirow{6}{*}{$\begin{array}{l}\text { Fruits, nuts and } \\
\text { seeds, olives }\end{array}$} & Fruits & -0.5 & 0.4 & -0.5 & 1.4 & 136 \\
\hline & Fruit compote & -1.7 & 0.0 & -0.1 & 0.0 & -2 \\
\hline & Nuts, peanuts, seeds & -2.1 & 0.0 & -0.1 & 0.0 & -67 \\
\hline & Peanut butter, nut/seeds spread & 0.7 & 0.0 & 0.0 & 0.0 & -4 \\
\hline & Olives & -3.7 & - & 0.0 & -1.4 & -13 \\
\hline & & & 100.0 & & & \\
\hline \multirow[t]{10}{*}{$\begin{array}{l}\text { Dairy products and } \\
\text { substitutes }\end{array}$} & $\begin{array}{l}\text { Non fermented milk and milk } \\
\text { beverages }\end{array}$ & 0.4 & 0.2 & 0.5 & 0.8 & 187 \\
\hline & $\begin{array}{l}\text { Fermented milk, milk beverages } \\
\text { and yogurt }\end{array}$ & -0.2 & 0.0 & -0.1 & 0.0 & -3 \\
\hline & Milk substitutes & -3.0 & 0.0 & -0.3 & 0.0 & -19 \\
\hline & Yoghurt & 1.7 & 0.0 & 0.9 & 0.0 & 20 \\
\hline & Fromage blanc, petits suisses & -0.2 & 0.0 & 0.0 & 0.0 & -11 \\
\hline & $\begin{array}{l}\text { Cheeses (including spread } \\
\text { cheeses) }\end{array}$ & -0.9 & -1.0 & -0.3 & -0.9 & 70 \\
\hline & Unclassified creams & 0.0 & 0.0 & 0.0 & 0.0 & -16 \\
\hline & Dairy creams and creamers & -1.0 & -2.3 & 0.0 & -0.4 & 60 \\
\hline & Non dairy creams and creamers & -67.9 & 0.0 & -0.4 & 0.0 & -32 \\
\hline & Ice cream & -0.1 & 0.0 & 0.0 & 0.0 & -2 \\
\hline \multirow[t]{6}{*}{$\begin{array}{l}\text { Cereals and cereal } \\
\text { products }\end{array}$} & $\begin{array}{l}\text { Flours, starches, flakes, } \\
\text { semolina }\end{array}$ & 5.0 & 4.9 & 0.1 & 0.7 & 69 \\
\hline & Pasta, rice, other grain & 4.0 & 6.0 & 1.9 & 10.5 & -7 \\
\hline & Bread & 0.8 & 1.2 & 1.0 & 3.1 & 118 \\
\hline & Crispbread, rusks & -0.2 & 0.0 & 0.0 & 0.0 & 1 \\
\hline & Breakfast cereals & -0.2 & 0.0 & 0.0 & 0.0 & -10 \\
\hline & Dough and pastry & 1.8 & 1.6 & 0.1 & 0.9 & -8 \\
\hline \multirow{9}{*}{$\begin{array}{l}\text { Meat, meat products } \\
\text { and substitutes }\end{array}$} & Unclassified and combined meat & -6.9 & 0.0 & -0.2 & 0.0 & -23 \\
\hline & $\begin{array}{l}\text { Unclassified, mixed and other } \\
\text { mammals }\end{array}$ & 58.2 & 26.1 & 2.2 & 8.0 & 355 \\
\hline & Beef & -9.2 & -2.7 & -1.1 & -1.9 & -307 \\
\hline & Veal & -8.9 & 0.0 & 0.0 & 0.0 & -5 \\
\hline & Pork & 12.1 & 0.0 & 1.6 & 0.0 & 177 \\
\hline & Mutton/lamb & 36.7 & 0.0 & 0.2 & 0.0 & 27 \\
\hline & Chicken, hen & 5.7 & 12.0 & 0.9 & 9.2 & -208 \\
\hline & Turkey, young turkey & -17.2 & 0.0 & -0.1 & 0.0 & -8 \\
\hline & Game & -10.2 & 0.0 & 0.0 & 0.0 & -1 \\
\hline
\end{tabular}




\begin{tabular}{|c|c|c|c|c|c|c|}
\hline \multirow[b]{2}{*}{ Food Groups } & \multirow[b]{2}{*}{ Food sub-groups } & \multicolumn{2}{|c|}{$\begin{array}{l}\text { Percentage } \\
\text { Difference }\end{array}$} & \multicolumn{2}{|c|}{$\begin{array}{l}\text { Amount } \\
\text { Difference }\end{array}$} & \multirow[b]{2}{*}{ Occurrence } \\
\hline & & Mean & P95 & Mean & P95 & \\
\hline & Hot processed meat & 1.0 & 3.2 & 0.3 & 3.2 & 36 \\
\hline & Cold processed meat & -0.1 & -1.5 & 0.0 & -1.0 & 42 \\
\hline & Hot meat substitutes & -16.5 & 0.0 & -0.2 & 0.0 & -35 \\
\hline & Cold meat substitutes & -0.6 & 0.0 & 0.0 & 0.0 & -1 \\
\hline \multirow[t]{4}{*}{$\begin{array}{l}\text { Fish, shellfish and } \\
\text { amphibians }\end{array}$} & $\begin{array}{l}\text { Unclassified and combined fish } \\
\text { products }\end{array}$ & -30.4 & 0.0 & 0.0 & 0.0 & -1 \\
\hline & Fish & -3.4 & 0.0 & -0.4 & 0.0 & -40 \\
\hline & Crustaceans, molluscs & -4.1 & 0.0 & -0.1 & 0.0 & 2 \\
\hline & Fish products & -0.9 & $100 . \overline{0}$ & 0.0 & -0.4 & -3 \\
\hline $\begin{array}{l}\text { Eggs and egg } \\
\text { products }\end{array}$ & Eggs & 2.6 & 6.2 & 0.3 & 3.1 & 88 \\
\hline \multirow[t]{5}{*}{ Fats and oils } & Unclassified and combined fats & 78.1 & 38.7 & 1.1 & 3.2 & 1758 \\
\hline & Vegetable oils & -3.7 & -2.8 & -0.1 & -0.4 & -403 \\
\hline & Butter & -2.9 & -2.3 & -0.1 & -0.3 & -101 \\
\hline & Margarines and cooking fats & -2.8 & -2.9 & -0.4 & -1.2 & -589 \\
\hline & $\begin{array}{l}\text { Other animal fats (including fish } \\
\text { oils) }\end{array}$ & -2.2 & 0.0 & 0.0 & 0.0 & -3 \\
\hline \multirow{8}{*}{$\begin{array}{l}\text { Sugar and } \\
\text { confectionery }\end{array}$} & Sugar & -0.5 & 0.0 & 0.0 & 0.0 & -54 \\
\hline & Jam, jelly, marmelade & 0.0 & 0.0 & 0.0 & 0.0 & -2 \\
\hline & Honey & -1.2 & -11.1 & 0.0 & -0.7 & -14 \\
\hline & Other sweet spread & 0.1 & 0.0 & 0.0 & 0.0 & -4 \\
\hline & Syrup & 1.1 & 0.0 & 0.0 & 0.0 & -1 \\
\hline & Unclassified and other chocolate & -0.1 & 0.0 & 0.0 & 0.0 & -2 \\
\hline & $\begin{array}{l}\text { Chocolate spread and chocolate } \\
\text { powder }\end{array}$ & -0.1 & 0.0 & 0.0 & 0.0 & -2 \\
\hline & Confectionery non chocolate & -0.1 & 0.0 & 0.0 & 0.0 & 11 \\
\hline $\begin{array}{l}\text { Cakes and sweet } \\
\text { biscuits }\end{array}$ & Cakes, pies, pastries, puddings & 0.0 & 0.0 & 0.0 & 0.0 & -4 \\
\hline \multirow[t]{4}{*}{$\begin{array}{l}\text { Non alcoholic } \\
\text { beverages }\end{array}$} & $\begin{array}{l}\text { Unclassified and combined non } \\
\text { alc. Drinks }\end{array}$ & -1.1 & 0.0 & -0.1 & 0.0 & -31 \\
\hline & Fruit and vegetable juices & 2.2 & 0.0 & 1.2 & 0.0 & 160 \\
\hline & Carbonated/soft/isotonic drinks & 0.0 & 0.0 & 0.0 & 0.0 & -17 \\
\hline & Waters & 0.4 & 0.0 & 0.0 & 0.0 & 304 \\
\hline \multirow[t]{8}{*}{$\begin{array}{l}\text { Condiments, spices, } \\
\text { sauces and yeast }\end{array}$} & $\begin{array}{l}\text { Unclassified or combined } \\
\text { condiments }\end{array}$ & -2.8 & 0.0 & 0.0 & 0.0 & -1 \\
\hline & Other and mixed sauces & -6.7 & -4.9 & -0.9 & -2.9 & -133 \\
\hline & Tomato sauces & 3.8 & 0.6 & 0.3 & 0.2 & 29 \\
\hline & $\begin{array}{l}\text { Dressing sauces, mayonnaises } \\
\text { and similar }\end{array}$ & -2.1 & -0.5 & -0.2 & -0.2 & -139 \\
\hline & Mayonnaise based spreads & -0.6 & 0.0 & 0.0 & 0.0 & -8 \\
\hline & Spices, herbs and flavourings & -47.1 & 0.0 & 0.0 & 0.0 & -21 \\
\hline & $\begin{array}{l}\text { Unclassified and combined } \\
\text { condiments }\end{array}$ & 36.7 & 14.9 & 0.7 & 1.6 & 339 \\
\hline & Vinegar & -21.1 & -48.4 & 0.0 & -0.4 & -34 \\
\hline \multirow[t]{2}{*}{ Soups and stocks } & Soups & -42.9 & -25.1 & -8.0 & -35.7 & -481 \\
\hline & Stocks & 21.6 & 11.4 & 5.2 & 16.2 & 21 \\
\hline \multirow[t]{2}{*}{ Miscellaneous } & Artificial sweeteners & 0.0 & 0.0 & 0.0 & 0.0 & -7 \\
\hline & Meal substitutes & -0.1 & 0.0 & 0.0 & 0.0 & -2 \\
\hline \multirow[t]{2}{*}{ Savoury snacks } & $\begin{array}{l}\text { Savoury snacks, biscuits and } \\
\text { crisps }\end{array}$ & -0.2 & 0.0 & 0.0 & 0.0 & -10 \\
\hline & Savoury filled buns, croissants & 0.0 & 0.0 & 0.0 & 0.0 & -1 \\
\hline
\end{tabular}




\section{Appendix 2a: The percentage and actual difference of the nutrient intake distribution of the total population between the combined scenario and the original data.}

\section{Percentage Difference Amount Difference}

\begin{tabular}{|c|c|c|c|c|c|c|c|c|}
\hline Nutrients & Mean & P5 & P50 & P95 & Mean & P5 & P50 & P95 \\
\hline Energy (kcal) & 0.2 & 1.4 & -0.3 & -0.1 & 3.82 & 16.17 & -5.44 & -4.11 \\
\hline Protein $(\mathrm{g})$ & 0.0 & 0.1 & 0.2 & 1.4 & 0.00 & 0.05 & 0.15 & 1.75 \\
\hline Carbonhydrates (g) & 0.6 & 0.6 & 0.7 & 0.6 & 1.31 & 0.74 & 1.55 & 2.45 \\
\hline Mono- and disaccharides (g) & -0.1 & 0.1 & -0.2 & -0.3 & -0.13 & 0.03 & -0.17 & -0.65 \\
\hline Fibre $(\mathrm{g})$ & -0.8 & -0.2 & -0.6 & 0.1 & -0.15 & -0.02 & -0.12 & 0.03 \\
\hline Fat $(\mathrm{g})$ & -0.1 & -0.6 & 0.3 & -0.8 & -0.01 & -0.02 & 0.04 & -0.20 \\
\hline SFA (g) & -0.5 & -0.4 & -0.7 & -0.8 & -0.14 & -0.04 & -0.20 & -0.41 \\
\hline TFA (g) & -1.1 & -2.5 & -1.2 & 0.3 & -0.01 & -0.01 & -0.01 & 0.01 \\
\hline ALA (g) & 0.2 & 4.2 & -0.1 & -1.6 & 0.00 & 0.02 & 0.00 & -0.06 \\
\hline DHA (mg) & -2.3 & 0.0 & -10.1 & -2.1 & -2.63 & 0.00 & -0.77 & -14.51 \\
\hline Calcium (mg) & -0.1 & 1.1 & 0.0 & -1.3 & -0.60 & 4.35 & 0.00 & -23.18 \\
\hline Iron (mg) & -0.8 & 0.4 & -0.9 & -0.5 & -0.08 & 0.02 & -0.09 & -0.08 \\
\hline Sodium (mg) & 0.4 & -1.2 & -0.1 & 1.3 & 9.11 & -12.82 & -2.99 & 54.34 \\
\hline Potassium (mg) & -0.5 & 0.3 & -1.1 & 0.2 & -16.48 & 4.17 & -33.81 & 7.90 \\
\hline Zinc (mg) & -0.2 & -0.3 & 0.1 & -1.2 & -0.02 & -0.01 & 0.01 & -0.21 \\
\hline Beta carotene $(\mu \mathrm{g})$ & -1.3 & 2.4 & 0.0 & -2.8 & -26.67 & 4.94 & -0.43 & -207.32 \\
\hline Retinol $(\mu \mathrm{g})$ & 0.2 & 2.1 & 0.1 & -0.2 & 1.15 & 2.75 & 0.60 & -3.62 \\
\hline Folate equivalents $(\mu \mathrm{g})$ & -0.9 & -0.1 & -1.2 & -0.2 & -2.09 & -0.15 & -2.84 & -0.80 \\
\hline Vitamin B1 (mg) & 1.8 & 1.0 & 0.8 & 3.6 & 0.02 & 0.00 & 0.01 & 0.07 \\
\hline Vitamin B2 (mg) & -0.2 & 0.6 & 0.0 & -0.6 & 0.00 & 0.00 & 0.00 & -0.02 \\
\hline Vitamin B3 (mg) & -0.4 & -0.8 & -1.3 & 0.2 & -0.08 & -0.06 & -0.21 & 0.06 \\
\hline Vitamin B6 (mg) & -0.5 & -0.2 & -1.4 & 0.7 & -0.01 & 0.00 & -0.02 & 0.02 \\
\hline Vitamin B12 $(\mu \mathrm{g})$ & -0.5 & -1.9 & -0.4 & 0.0 & -0.02 & -0.03 & -0.01 & 0.00 \\
\hline Vitamin C (mg) & -1.8 & -0.1 & -1.8 & -2.6 & -1.66 & -0.01 & -1.37 & -5.47 \\
\hline Vitamin D $(\mu \mathrm{g})$ & -0.2 & 0.0 & 0.0 & 0.6 & -0.01 & 0.00 & 0.00 & 0.04 \\
\hline Vitamin $\mathrm{E}(\mu \mathrm{g})$ & -0.6 & -1.1 & -0.7 & -0.1 & -0.07 & -0.05 & -0.08 & -0.02 \\
\hline
\end{tabular}


Appendix 2b: The percentage and actual difference of the nutrient intake distribution of the recipe population between the combined scenario and the original data.

\begin{tabular}{|c|c|c|c|c|c|c|c|c|}
\hline \multirow[b]{2}{*}{ Nutrients } & \multicolumn{8}{|c|}{ Percentage Difference Amount Difference } \\
\hline & Mean & P5 & P50 & P95 & Mean & P5 & P50 & P95 \\
\hline xcal) & 0.3 & 2.1 & -0.2 & 0.7 & 6.76 & 24.14 & 3.77 & 24.61 \\
\hline Protein $(g)$ & 0.0 & 0.3 & 0.0 & 1.2 & 0.00 & 0.13 & 0.03 & 1.59 \\
\hline Carbonhydrates (g) & 1.0 & 0.6 & 1.2 & 3.1 & 2.32 & 0.72 & 2.72 & 11.78 \\
\hline Mono- and disaccharides (g) & -0.2 & 0.1 & -0.1 & 0.0 & 0.22 & 0.03 & 0.09 & 0.09 \\
\hline Fibre (g) & -1.3 & -1.1 & -0.9 & -0.9 & 0.27 & 0.11 & 0.17 & 0.30 \\
\hline at $(\mathrm{g})$ & -0.3 & 0.6 & -0.2 & 1.1 & 0.23 & 0.22 & 0.17 & 1.60 \\
\hline SFA (g) & -0.8 & 0.1 & -1.2 & -2.4 & 0.24 & 0.01 & 0.34 & 1.32 \\
\hline TFA (g) & -2.0 & -5.2 & -2.4 & 0.6 & 0.02 & 0.01 & 0.02 & 0.01 \\
\hline ALA (g) & 0.3 & 4.7 & -0.6 & -2.1 & 0.01 & 0.03 & 0.01 & 0.07 \\
\hline DHA (mg) & -4.5 & 0.0 & -12.8 & -8.4 & 4.66 & 0.00 & 0.93 & 52.71 \\
\hline Calcium (mg) & -0.1 & 1.9 & 0.1 & -1.5 & 1.05 & 7.77 & 1.34 & 26.35 \\
\hline on (mg) & -1.3 & 0.7 & -1.5 & -0.7 & 0.13 & 0.03 & 0.14 & 0.12 \\
\hline Sodium (mg) & 0.7 & -1.6 & 0.1 & 3.6 & 16.13 & 18.19 & 2.68 & 150.66 \\
\hline Potassium (mg) & -0.9 & 0.2 & -2.0 & -0.7 & 29.18 & 3.30 & 61.16 & 535.97 \\
\hline Zinc (mg) & -0.3 & -0.7 & 0.0 & -1.6 & 0.03 & 0.04 & 0.00 & 0.27 \\
\hline Beta carotene $(\mu \mathrm{g})$ & -2.2 & 2.3 & 0.6 & -5.2 & 47.23 & 5.48 & 7.60 & 391.76 \\
\hline Retinol $(\mu \mathrm{g})$ & 0.3 & 2.7 & 0.2 & -0.6 & 2.03 & 3.64 & 0.81 & 10.60 \\
\hline Folate equivalents $(\mu \mathrm{g})$ & -1.5 & 0.6 & -2.2 & -1.1 & 3.70 & 0.62 & 5.00 & 4.59 \\
\hline Vitamin B1 (mg & 3.2 & 1.3 & 1.6 & 10.0 & 0.03 & 0.01 & 0.01 & 0.19 \\
\hline Vitamin B2 (mg) & -0.4 & 0.4 & 0.5 & -0.9 & 0.01 & 0.00 & 0.01 & 0.02 \\
\hline Vitamin B3 (mg) & -0.8 & -1.1 & -2.3 & 0.6 & 0.15 & 0.08 & 0.37 & 0.22 \\
\hline Vitamin B6 (mg) & -0.9 & -0.3 & -1.6 & 1.5 & 0.01 & 0.00 & 0.02 & 0.04 \\
\hline Vitamin B12 $(\mu \mathrm{g})$ & -0.9 & -2.7 & -0.6 & -1.7 & 0.04 & 0.04 & 0.02 & 0.15 \\
\hline Vitamin C (mg) & -3.2 & -3.9 & -2.9 & -3.2 & 2.93 & 0.96 & 2.21 & 6.68 \\
\hline Vitamin $\mathrm{D}(\mu \mathrm{g})$ & -0.3 & 3.1 & 0.3 & 0.4 & 0.01 & 0.02 & 0.01 & 0.03 \\
\hline Vitamin E $(\mu \mathrm{g})$ & -1.1 & -1.0 & -2.5 & 0.1 & 0.13 & 0.05 & 0.27 & 0.03 \\
\hline
\end{tabular}




\section{Appendix 3a: The percentage and actual difference of the nutrient intake distribution of the total population between the individual scenario and the original data.}

Percentage Difference Amount Difference

\begin{tabular}{|c|c|c|c|c|c|c|c|c|}
\hline Nutrients & Mean & P5 & P50 & P95 & Mean & P5 & P50 & P95 \\
\hline Energy (kcal) & 0.2 & 1.2 & -0.4 & 0.6 & 4.62 & 14.51 & 7.24 & 22.04 \\
\hline Protein (g) & 0.1 & -0.1 & 0.2 & 1.5 & 0.08 & 0.03 & 0.15 & 1.91 \\
\hline Carbonhydrates (g) & 0.5 & 0.9 & 0.7 & 0.6 & 1.24 & 1.11 & 1.55 & 2.45 \\
\hline Mono- and disaccharides (g) & -0.1 & 0.0 & -0.1 & -0.3 & 0.11 & 0.00 & 0.15 & 0.65 \\
\hline Fibre $(\mathrm{g})$ & -0.7 & -0.2 & -0.5 & 0.1 & 0.14 & 0.02 & 0.10 & 0.03 \\
\hline Fat $(g)$ & -0.1 & 0.4 & -0.2 & -0.1 & 0.05 & 0.16 & 0.14 & 0.09 \\
\hline SFA $(g)$ & -0.4 & -0.7 & -0.5 & -1.2 & 0.12 & 0.08 & 0.15 & 0.66 \\
\hline TFA (g) & -1.0 & -1.8 & -1.2 & 0.1 & 0.01 & 0.00 & 0.01 & 0.00 \\
\hline $\operatorname{ALA}(\mathrm{g})$ & 0.3 & 5.2 & -0.1 & -0.4 & 0.01 & 0.03 & 0.00 & 0.01 \\
\hline DHA (mg) & -2.0 & 0.0 & -7.4 & -2.5 & 2.28 & 0.00 & 0.56 & 16.56 \\
\hline Calcium (mg) & -0.1 & 1.1 & 0.3 & -1.2 & 0.56 & 4.30 & 2.86 & 20.44 \\
\hline Iron (mg) & -0.6 & 0.4 & -0.9 & -0.3 & 0.06 & 0.02 & 0.09 & 0.06 \\
\hline Sodium (mg) & 0.3 & -1.0 & 0.0 & 1.2 & 7.76 & 10.72 & 0.25 & 47.90 \\
\hline Potassium (mg) & -0.4 & 0.3 & -0.8 & 0.0 & 13.99 & 4.20 & 25.64 & 0.00 \\
\hline Zinc (mg) & -0.1 & -0.3 & 0.0 & -1.2 & 0.01 & 0.01 & 0.00 & 0.21 \\
\hline Beta carotene $(\mu g)$ & -1.6 & 1.8 & -0.4 & -5.2 & 32.98 & 3.74 & 5.09 & 382.04 \\
\hline Retinol ( $\mu \mathrm{g})$ & 0.2 & 0.0 & 0.2 & -0.2 & 1.08 & 0.00 & 0.63 & 3.62 \\
\hline Folate equivalents $(\mu \mathrm{g})$ & -0.7 & 0.0 & -1.2 & -0.5 & 1.75 & 0.05 & 2.84 & 2.06 \\
\hline Vitamin B1 (mg) & 1.5 & 0.7 & 0.7 & 2.7 & 0.02 & 0.00 & 0.01 & 0.06 \\
\hline Vitamin B2 (mg) & -0.2 & 0.6 & 0.0 & -0.6 & 0.00 & 0.00 & 0.00 & 0.02 \\
\hline Vitamin B3 (mg) & -0.2 & -0.7 & -0.7 & 0.1 & 0.04 & 0.05 & 0.12 & 0.03 \\
\hline Vitamin B6 (mg) & -0.3 & 0.0 & -0.9 & 1.1 & 0.01 & 0.00 & 0.01 & 0.03 \\
\hline Vitamin B12 $(\mu \mathrm{g})$ & -0.4 & -1.2 & -0.6 & -0.4 & 0.02 & 0.02 & 0.02 & 0.03 \\
\hline Vitamin C (mg) & -1.6 & 1.0 & -1.9 & -3.1 & 1.46 & 0.23 & 1.46 & 6.31 \\
\hline Vitamin D $(\mu \mathrm{g})$ & -0.2 & -0.1 & 0.1 & 0.0 & 0.00 & 0.00 & 0.00 & 0.00 \\
\hline Vitamin $E(\mu g)$ & -0.4 & -0.7 & -0.6 & -0.1 & 0.05 & 0.03 & 0.07 & 0.02 \\
\hline
\end{tabular}


Appendix 3b: The percentage and actual difference of the nutrient intake distribution of the total population between the modified scenario and the original data.

Percentage Difference Amount Difference

\begin{tabular}{|c|c|c|c|c|c|c|c|}
\hline Nutrients & Mean & P5 & P50 & P95 & Mean P5 & P50 & P95 \\
\hline Energy (kcal) & -0.1 & -0.5 & 0.0 & -0.3 & 3.065 .30 & 0.00 & 11.02 \\
\hline Protein (g) & -0.2 & 0.0 & -0.4 & -0.5 & 0.190 .02 & 0.28 & 0.69 \\
\hline Carbonhydrates (g) & -0.1 & 0.0 & 0.0 & 0.0 & 0.140 .00 & 0.00 & 0.00 \\
\hline Mono- and disaccharides (g) & 0.0 & 0.1 & -0.1 & -0.3 & 0.050 .03 & 0.15 & 0.65 \\
\hline Fibre (g) & -0.2 & -0.2 & -0.2 & 0.0 & 0.040 .02 & 0.03 & 0.00 \\
\hline Fat $(g)$ & -0.2 & -0.1 & -0.1 & -0.1 & 0.190 .05 & 0.04 & 0.09 \\
\hline SFA (g) & -0.1 & 0.6 & -0.1 & 0.0 & 0.050 .08 & 0.03 & 0.00 \\
\hline TFA (g) & -0.2 & -1.1 & -0.4 & 0.1 & 0.000 .00 & 0.00 & 0.00 \\
\hline $\operatorname{ALA}(\mathrm{g})$ & -0.2 & 0.1 & -0.1 & -1.2 & 0.000 .00 & 0.00 & 0.04 \\
\hline DHA (mg) & -0.4 & 0.0 & -2.7 & 0.0 & 0.440 .00 & 0.21 & 0.00 \\
\hline Calcium (mg) & -0.1 & -0.3 & -0.2 & -0.6 & 0.841 .13 & 1.71 & 9.92 \\
\hline Iron (mg) & -0.3 & -0.3 & -0.1 & -0.3 & $0.03 \quad 0.02$ & 0.01 & 0.06 \\
\hline Sodium (mg) & -0.1 & -0.6 & -0.2 & 0.0 & 3.017 .10 & 4.36 & 1.06 \\
\hline Potassium (mg) & -0.2 & 0.0 & -0.4 & 0.0 & 6.040 .63 & 11.25 & 0.00 \\
\hline Zinc (mg) & -0.2 & 0.0 & -0.2 & -1.0 & 0.020 .00 & 0.02 & 0.16 \\
\hline Beta carotene $(\mu \mathrm{g})$ & 0.0 & -1.0 & 0.2 & 0.5 & 0.512 .07 & 2.29 & 35.07 \\
\hline Retinol $(\mu \mathrm{g})$ & 0.0 & 1.0 & -0.1 & -0.2 & 0.321 .28 & 0.50 & 3.62 \\
\hline Folate equivalents $(\mu \mathrm{g})$ & -0.3 & -0.7 & -0.5 & 0.4 & 0.610 .77 & 1.06 & 1.90 \\
\hline Vitamin B1 (mg) & 0.2 & -0.2 & 0.0 & 0.1 & 0.000 .00 & 0.00 & 0.00 \\
\hline Vitamin B2 (mg) & -0.1 & -0.1 & -0.1 & -0.1 & 0.000 .00 & 0.00 & 0.00 \\
\hline Vitamin B3 (mg) & -0.3 & 0.0 & -0.9 & 0.0 & 0.060 .00 & 0.14 & 0.01 \\
\hline Vitamin B6 (mg) & -0.3 & 0.4 & -0.8 & 0.0 & 0.000 .00 & 0.01 & 0.00 \\
\hline Vitamin B12 ( $\mu \mathrm{g})$ & -0.2 & -1.0 & -0.3 & 0.0 & 0.010 .01 & 0.01 & 0.00 \\
\hline Vitamin C (mg) & -0.4 & -2.5 & -0.1 & -0.1 & 0.330 .58 & 0.09 & 0.25 \\
\hline Vitamin D $(\mu \mathrm{g})$ & -0.1 & 0.0 & -0.1 & 0.3 & 0.000 .00 & 0.00 & 0.02 \\
\hline Vitamin $E(\mu g)$ & -0.3 & -0.4 & -0.2 & -0.1 & 0.040 .02 & 0.02 & 0.03 \\
\hline
\end{tabular}





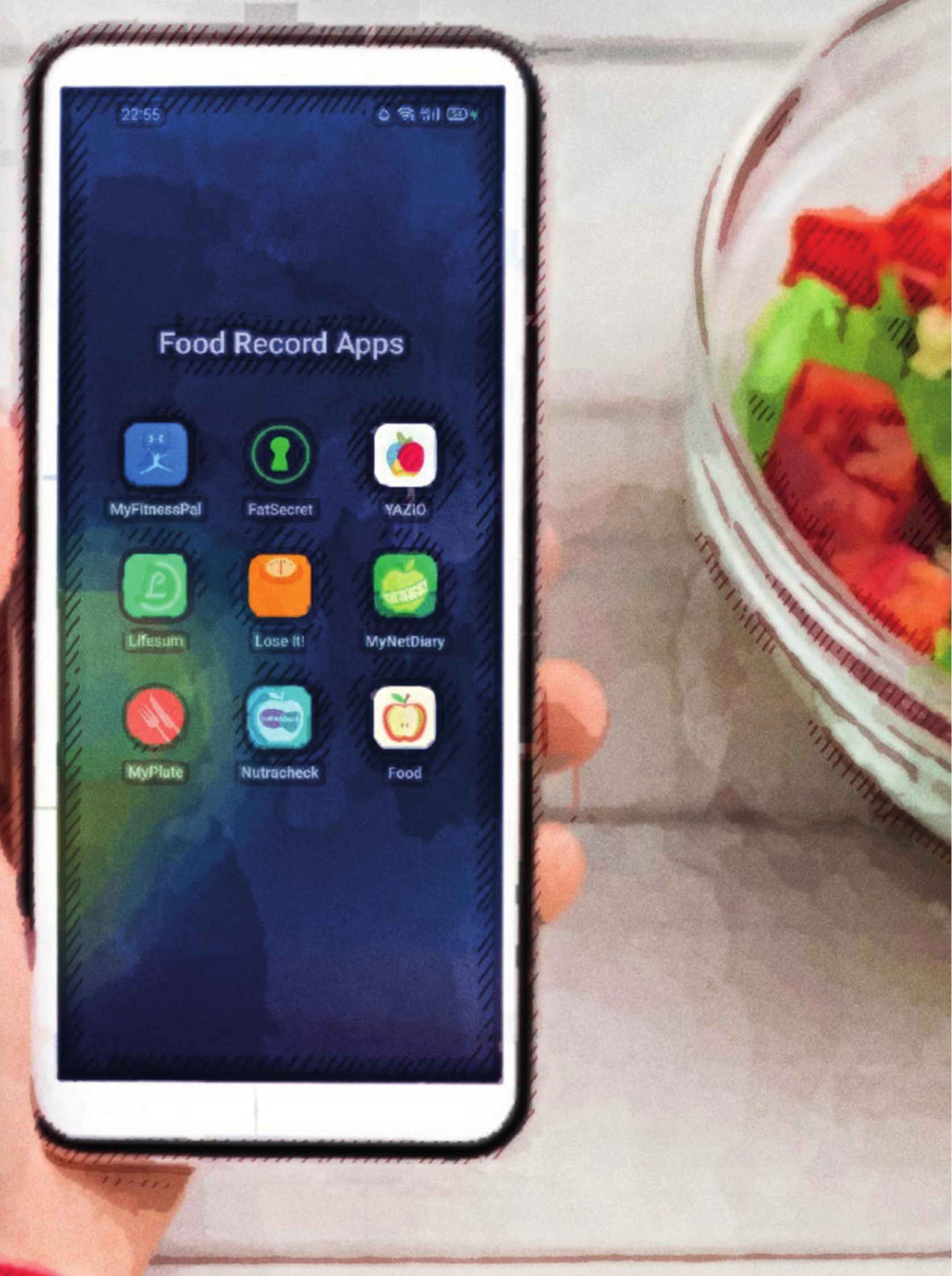




\section{Chapter 4}

Evaluation of the Recipe Function in Popular Dietary Smartphone Applications, with Emphasize on Features Relevant for Nutrition Assessment in Large-Scale Studies

Liangzi Zhang, Eline Nawijn, Hendriek Boshuizen, Marga Ocké Nutrients 2019, $11(1), 200$ 


\begin{abstract}
Nutrient estimations from mixed dishes require detailed information collection and should account for nutrient loss during cooking. This study aims to make an inventory of recipe creating features in popular food diary apps from a research perspective and to evaluate their nutrient calculation. A total of 12 out of 57 screened popular dietary assessment apps included a recipe function and were scored based on a pre-defined criteria list. Energy and nutrient content of three recipes calculated by the apps were compared with a reference procedure, which takes nutrient retention due to cooking into account. The quality of the recipe function varies across selected apps with a mean score of 3.0 (out of 5). More relevant differences (larger than 5\% of the Daily Reference Intake) between apps and the reference were observed in micronutrients (49\%) than in energy and macronutrients $(20 \%)$. The primary source of these differences lies in the variation in food composition databases underlying each app. Applying retention factors decreased the micronutrient contents from $0 \%$ for calcium in all recipes to more than $45 \%$ for vitamins B6, B12, and folate in one recipe. Overall, recipe features and their ability to capture true nutrient intake are limited in current apps.
\end{abstract}




\section{Introduction}

When assessing the dietary intake of a large population, an accurate dietary assessment plays a fundamental role [1]. Self-report dietary assessment methods, such as 24-hour dietary recall (24HDR), dietary record (DR), and food frequency questionnaire (FFQ), are commonly used to assess food consumption at both individual and population level [2]. Since underreporting, overreporting, misreporting, and interviewer bias can occur in those methods [3-5], assessing dietary intake with a high level of accuracy continues to be a major challenge in nutritional epidemiology and monitoring [6,7]. Moreover, cumbersome procedures of collecting details of foods are time-consuming and are associated with a high burden for both the respondent and the researcher [8]. This is especially the case for 24HDR and DR, which are open methods, and for which repeated measurements are needed to estimate usual dietary intake [9]. The burden laid on respondents can also lead to a low response rate, which may lead to bias in the survey results and diminish the representativeness of the sample [10].

Progress in Information and Communication Technology (ICT) in the past few decades has led to investigations into innovative strategies to overcome drawbacks of traditional pen-andpaper and interviewer-based dietary assessment methods [11,12]. One such innovative strategy is the use of mobile applications (apps) on smartphones for a dietary record. In the last decade, an increase in the number of smartphone users has led to a proliferation of mobile applications (apps) [13]. A popular category within all these apps are the health and fitnessrelated apps [14], mostly aimed at supporting dietary change and weight management $[15,16]$. Those apps usually include a food diary function, in which users can record the foods consumed and the consumed quantities. Apart from searching in a pre-defined food and beverage list and selecting pre-defined portion sizes [17], various features are available to help identify consumed foods, estimate portion size, and decrease the burden of food entering. Examples of those features are image-based food recognition and barcode scanner. Their potential on reducing the respondents' burden, decreasing the effort of multiple selfadministrations and on improving food recording accuracy have been investigated in both experimental and observational epidemiological studies, and have shown some promising results $[6,18]$. However, the knowledge on the performance of other specific features is still limited [19].

One feature of food diary apps is the recipe function for entering mixed dishes prepared at home. These are dishes consisting of multiple foods, with specific food preparation and often with cooking involved. For user-friendliness, the recipe function should be structured in a way that could easily guide the users in recording necessary information of a recipe. It should be able to assess the recipe intake of an individual, while mixed dishes are often prepared for more than one person [19]. Furthermore, for a better estimation of nutrient intake, an accurate 
recipe calculation should take nutrient loss of ingredients during cooking and food processing into account [20].

Some food diary apps have introduced a recipe function through the recent years $[21,22]$. The effectiveness of these recipe functions in capturing the food consumption and nutrient intake has not been fully evaluated. Moreover, the question whether the features of available recipe functions are also appropriate for dietary assessment as part of large-scale studies remains unanswered. Therefore, the aim of this study was to make an inventory of recipe function features in apps that could facilitate the estimation of nutrient intake of a large population. Furthermore, another aim was to evaluate the accuracy of the recipe function in capturing nutrient intake of popular dietary assessment apps by comparing their nutrient calculation with a standard calculation procedure.

\section{Materials and Methods}

The starting point for app selection was an identification of dietary assessment smartphone apps in the Health \& Fitness category of iTunes App Store and Google Play Store in the Netherlands between 15th and 23rd of October 2016. This selection was performed by Maringer et al. [20] and resulted in the identification of 176 dietary assessment apps. Further screening was performed in August 2017. Inclusion of a subselection of apps for this study required the app to meet the following criteria: (1) user rating $>3$ in iTunes App Store and Google Play Store, (2) user rating count $>500$ in iTunes App Store and Google Play Store, (3) $>10,000$ downloads in the both stores, (4) a recipe function which was freely available, actually present and functional. A recipe function was defined as "a functionality in which the user can create a mixed dish by entering and specifying the amount of each ingredient within the dish" $[23,24]$. Each app underwent initial screening based on descriptions and associated images in the app stores to check for the presence of a recipe function. Apps were downloaded onto a OnePlus 3T smartphone running Android 7.1.1 and a Huawei Mate 8 running EMUI 5.0.1 for analysis. The apps were checked manually to confirm whether a recipe function was freely available, actually present, and functional. Basic descriptive information about the apps was identified, such as app name, version number, operating platforms, number of installs, ratings, whether they can synchronize with their website, and country of origin. Subsequently, the recipe function of the selected apps was evaluated.

To our knowledge, no widely accepted standard evaluation of the quality of the recipe function of apps exists. Therefore, a criteria list was made for evaluating features in the individual recipe function of apps. For each feature on the criteria list a rubric of assessment was created with a 1 (low)-5 (high) scoring scale. The criteria list and assessment rubric were modified upon findings from a pilot scoring and feedback from two nutritionists and three dietitians with different specializations. The criteria list and assessment include the following aspects of creating an individual recipe: options in searching ingredients, ways to 
record relevant information of the recipe, whether raw or cooked ingredients could be selected, consumed amount for both ingredients and the whole recipe, energy and nutrient expression, and whether the recipe could be saved and edited later (Table 1). Two researchers scored all the selected apps according to the criteria list independently. Inconsistent scores among the two researchers were discussed to reach agreed final scores. For scoring the criterion whether both raw and cooked foods are available in the food list, nine foods from the three most frequently used Dutch recipes (explained in next paragraph) were entered in each app (kale, potato, milk, mushroom, onion, salami, beef, pepper, and tomato).

To be able to evaluate the accuracy of energy and nutrient content estimations, three recipes were entered into the individual recipe function of each app. The selection of recipes was performed by exploring the most frequent reported recipes in the Dutch diet using the data of the Dutch National Food Consumption Survey (DNFCS) 2007-2010 [25]. Three recipes with different preparation methods, like stewing, baking, and frying, were chosen from the twenty most frequently consumed recipes. The chosen recipes were boerenkool stamppot (mashed potato with kale), pizza with salami, tomato, and mushrooms, and hachee (a traditional Dutch stew based on beef and onions). Raw ingredients of the recipes were entered in the selected apps and a set of rules for entering ingredients were followed, in case the exact match of food items or amount indications could not be found across apps. If available, energy, macro- and micronutrient values of the recipe were obtained based on the displayed nutrient content in the app. For those apps where the nutrient contents were not shown at the recipe level, values from ingredients of a recipe were added up by researchers. Then, nutrient contents from the apps were compared with nutrient contents derived from the Dutch food composition database (NEVO) [26]. To account for nutrient loss due to cooking, retention factors suggested by the European Food Information Resource [27] were applied to the nutrients derived by NEVO, see complete calculation in Supplementary Material (Table S1S11). A retention factor larger than 0 and lower than 1 implied nutrient loss due to cooking. A retention factor of 1 was used for energy and macronutrients for all ingredients in all recipes since they were not easily affected by cooking. Next to energy and macronutrient, micronutrients such as sodium, potassium, vitamin A represented as retinol equivalent (RE), vitamin C, calcium, vitamin E, vitamin B1, vitamin B2, vitamin B6, vitamin B12, and folate were selected for comparison between apps and the reference measure. Of these, sodium, potassium, and vitamin $\mathrm{E}$ had a retention factor of 1 for all ingredients in the three recipes mentioned above, hence, were deleted from analysis. Calcium also had a retention factor of 1 , but was maintained in the analysis as an example.

General characteristics of the 12 evaluated dietary assessment apps with recipe function were summarized. For each app, the mean score and standard deviation over all nine criteria was calculated (see Table 1). The mean and standard deviation of scores across apps were calculated for each criterion. Energy and nutrient content estimations of the three recipes for 
each app were analyzed using descriptive statistics. For nutrients with retention factor of 1 , a direct comparison could be made with the nutrient contents derived from NEVO combining nutrient contents of raw ingredients in the appropriate amounts. For the micronutrients with retention factors below 1, the reference was the NEVO nutrient contents of the raw ingredients after applying the relevant retention factors. For showing the effect of the retention factors, a comparison with NEVO nutrient contents of raw ingredients without applying retention factors was also made. A difference in values between apps and the reference of more than 5\% from the Daily Reference Intake (DRI) for adults was considered out of range [28].

To visualize the correlation between apps and nutrients, a principal component analysis (PCA) was conducted for each recipe separately with energy and macronutrients divided by their DRIs being set as variables. The first two principal components represent the most variation. This was done for energy and macronutrients only, since only 3 apps showed information on absolute amounts of micronutrients. The descriptive statistics were calculated using Excel 2016 software and the PCA was conducted in R version 3.5.0 (The R Foundation for Statistical Computing, Vienna, Austria). 


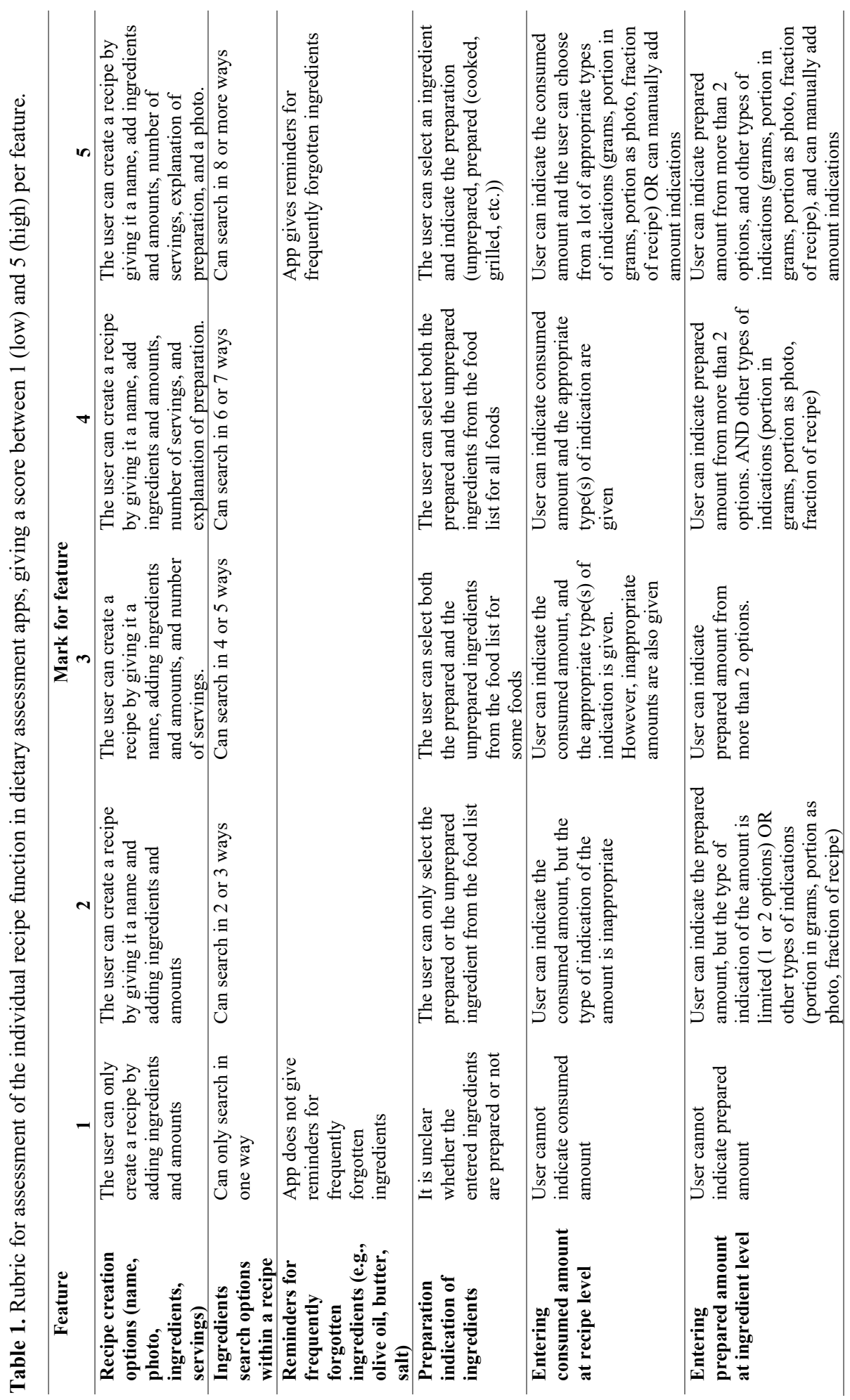




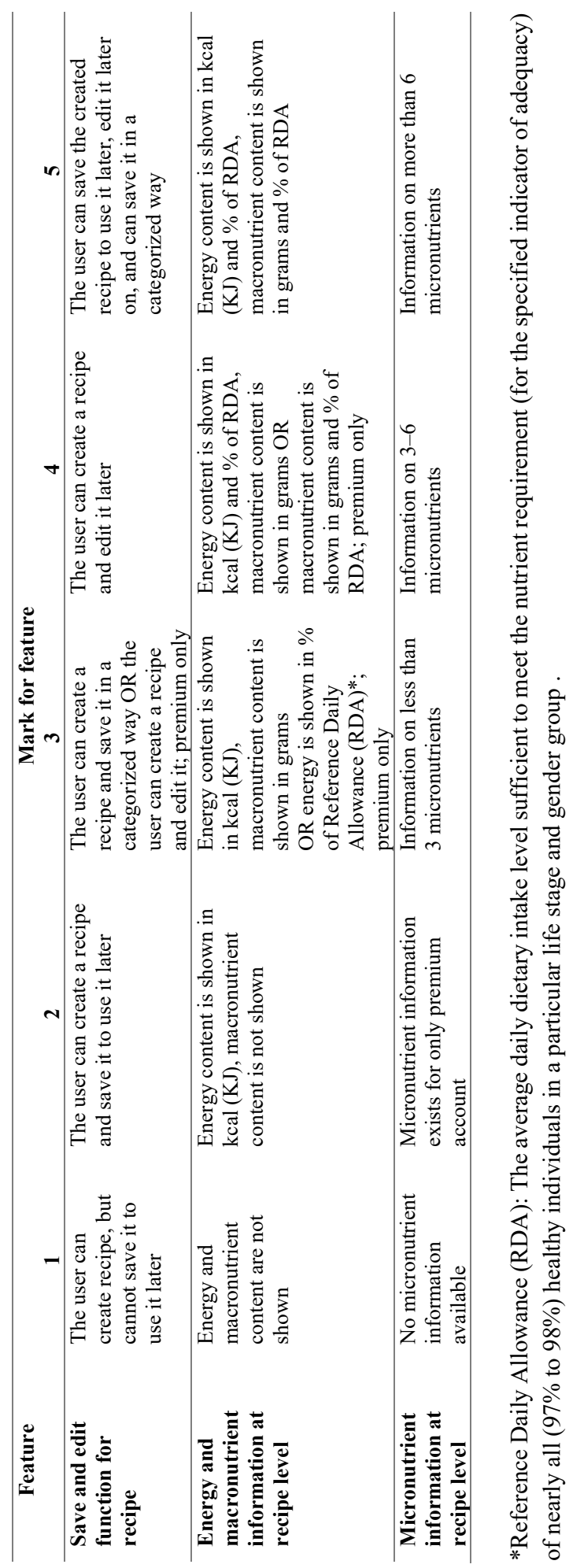




\section{Results}

\subsection{App Selection}

The starting point was a selection of 176 popular dietary assessment apps with food recording and available in English identified by Maringer et al. [21]. Then, apps were further narrowed down, with inclusion criteria of a user rating $>3$ in the iTunes App Store and Google Play Store, a user rating count $>500$ in iTunes App Store and Google Play Store, $>10,000$ downloads in the Google Play Store, and a claimed recipe function in the app description. After manually checking for the presence of an individual recipe function in 30 included apps, 17 apps were excluded from further evaluation because of dysfunction of the app, the absence or dysfunctionality of a recipe function, or the inability to use the app due to requirements of a membership. After final exclusion of one app with a non-functioning individual recipe function, a total of 12 apps (21\% of 57) were selected for evaluation in detail (Figure 1). 


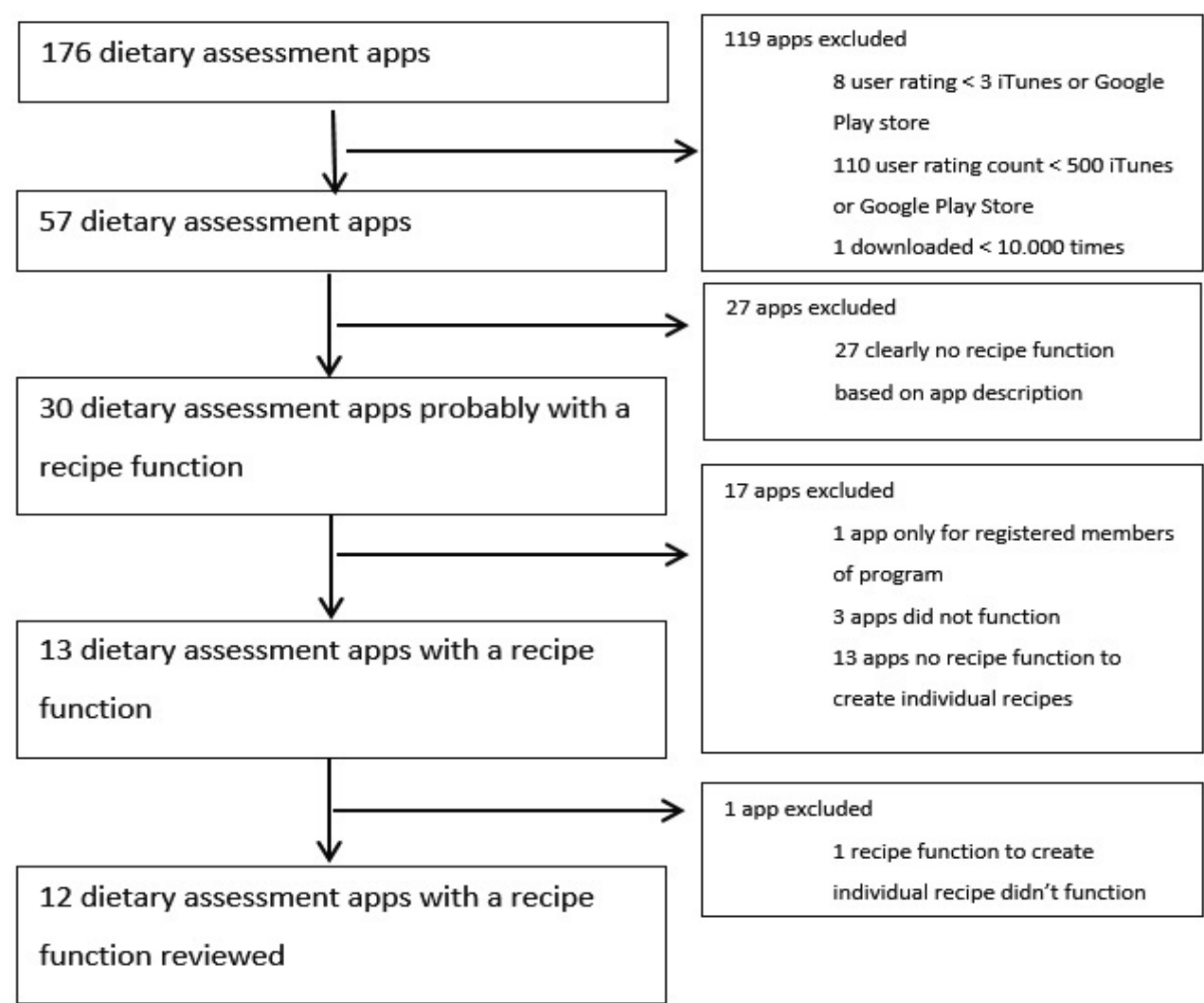

Figure 1. Flow diagram of selection procedure of dietary assessment apps with recipe function showing the number of apps included or excluded.

General characteristics of the remaining 12 apps can be found in Table 2. All apps operated on an Android platform, whereas IOS ranked as the second most-prevalent platform (10 apps). The highest number of installs was 50 million with 1844 thousand ratings for MyFitnessPal, the lowest was 100 thousand installs and 2000 ratings for Nutracheck. The rating scores among the apps ranged from 4.2 to 4.6 with the maximum score of 5.0. Four apps were made by US companies, two apps were made in Germany, and the rest of apps were made in other countries, mostly northwest Europe.

\subsection{Qualitative Recipe Function Assessment}

Agreed scores given to recipe functions of each app are shown in Table 3. Mean overall score of both apps and criteria was 3.0 (out of 5.0). The app Calories! had the highest score for its recipe function with an average score of 3.9 however, in contrast, Calories! had a rating score 
and number of installations at the lower range compared to other apps (Table 2). MyPlate and Health Infinity, on average, had the lowest scores of 2.2 and 2.3, respectively.

The apps that had relative higher popularity, such as MyFitnessPal, Lose It!, Lifesum, and MyPlate, did not have any criterion that scored 5, while Calories! was achieved a score of 5 three times. Health Infinity scored 1 most often (three times) compared to other apps.

Specifically, most of the evaluated apps could save a self-created recipe and edit it later, hence, this criterion ranked the highest (mean $=4.3$ ) compared to other criteria. None of the apps included reminders for frequently forgotten ingredients, therefore, all apps scored 1 for that criterion. The available options that existed for searching ingredients for recipes included text search, barcode scanning, voice record, recent/frequent/saved food, create new food, choose from categories, and choose from a list of all food in alphabetic order. The number of options ranged from 2 to 6 , where half of the apps had only 2 to 3 options, while only Nutracheck had all 6 options. The most frequently adopted options were search in a textbox and barcode scanning. FatSecret and Virtuagym Food had four searching options for food entering, but only two options for adding ingredients to recipes. In terms of options in searching raw or cooked foods, nearly all apps had both raw and cooked options for all or at least some foods in their dataset (mean = 3.3). An exception was The Secret of Weight, where, for the most foods, the text indicated raw while the picture showed cooked foods. In terms of indicating consumed amount in both ingredients and recipes, in Calories!, one could manually add a new serving unit to ingredients but not in recipes whereas, in Virtuagym Food, this was the other way around. Health Infinity had no options to chooe the amount of recipe consumed (scored as 1 ), and had only one built-in option when choosing the amount of ingredients. In terms of macronutrient information, Calories! was the only app that had energy and macronutrients expressed as both absolute amounts (mg, $\mu \mathrm{g}$, etc.) and \% of Recommended Daily Allowance (RDA). Most apps had energy and macronutrients shown only in absolute amounts. Since only four apps showed micronutrient for recipes, the average score for micronutrient availability ranked the second lowest with a score of 2.7. Among the apps with micronutrients, Calories! and MyNetDiary had both absolute amounts and \% RDA for more than six micronutrients, while Virtuagym Food had only actual amounts. MyFitnessPal had only \% RDA of less than six micronutrients.

\subsection{Accuracy of Energy and Macronutrient Content Estimations}

The differences in energy and macronutrient content estimations of the three recipes between the 12 popular dietary assessment apps and the value derived from NEVO are presented in Table 4. Macronutrient contents for both recipes and ingredients were not available in The Secret of Weight. Heterogeneity in differences was observed between recipes and between nutrients. Pizza had fewer differences $>5 \%(n=7)$ in the DRI as compared to boerenkool stamppot $(n=10)$ and hachee $(n=12)$. Carbohydrates $(n=2)$ and energy $(n=3)$ contents 
had fewer differences $>5 \%$ in the DRI than protein $(n=13)$ and fat $(n=11)$. In total, around $20 \%$ of the differences were $>5 \%$ DRI. Most apps underestimated the macronutrient content in boerenkool stamppot and pizza, while this was not observed in hachee.

With 7 out of 12, Nutracheck had the most discrepancies $>5 \%$ in the DRI compared to the reference, mainly caused by a discrepancy in fat and protein contents. YAZIO and Lifesum only had one difference of more than 5\%. Health Infinity had lower protein contents in all three recipes, whereas Lose It! had lower fat in all three recipes. Virtuagym Food and YAZIO had similar patterns in all recipes, and both had lower fat in hachee as outliers. MyNetDiary had all macronutrients being out of range once, including a lower carbohydrate, lower protein, and higher fat in three recipes, respectively. In Figure 2, apps are plotted against the first and second principal component of all differences in macronutrient contents. Macronutrients plotted further from the center indicate a larger variance. Apps situated in the same direction with a certain nutrient indicate an overestimation of the nutrient and vice versa. Nutracheck laid outside compared to other apps for all three recipes. MyFitnessPal was the only app without discrepancies of more than $5 \%$. Therefore, it was located around the center of the graph in all three recipes. 
Table 2. General characteristics, such as platforms available, number of installs on Google Play Store, user rating on Google Play Store and country of twelve popular dietary assessment apps with a recipe function $(n=12)$.

\begin{tabular}{|c|c|c|c|c|c|}
\hline & App Name (Version) & Platforms & $\begin{array}{l}\text { Installs } \\
\text { Google } \\
\text { Play Store } \\
\text { (Million) }\end{array}$ & $\begin{array}{l}\text { Rating } \\
\text { Google Play } \\
\text { Store (\# } \\
\text { Ratings/1000) }\end{array}$ & Country \\
\hline 1 & MyFitnessPal (18.6.0) & $\begin{array}{l}\text { Android, IOS, } \\
\text { Windows Phone }\end{array}$ & $50-100$ & $4.6(1844)$ & USA \\
\hline 2 & FatSecret (7.8.27) & $\begin{array}{l}\text { Android, IOS, } \\
\text { Windows Phone, } \\
\text { Watch OS, } \\
\text { Blackberry OS }\end{array}$ & $10-50$ & $4.4(223)$ & Australia \\
\hline 3 & YAZIO (4.0.1) & Android, IOS & $5-10$ & $4.6(109)$ & Germany \\
\hline 4 & Lose It! (9.4.5) & Android, IOS & $5-10$ & $4.4(68)$ & USA \\
\hline 5 & Lifesum (6.2.4) & $\begin{array}{l}\text { Android, IOS, } \\
\text { Watch OS, Android } \\
\text { Wear, }\end{array}$ & $5-10$ & $4.4(165)$ & Sweden \\
\hline 6 & MyPlate (3.2.2) & $\begin{array}{l}\text { Android, IOS, } \\
\text { Watch OS }\end{array}$ & $1-5$ & $4.6(22)$ & USA \\
\hline 7 & MyNetDiary (6.4.7) & $\begin{array}{l}\text { Android, IOS, } \\
\text { Watch OS }\end{array}$ & $1-5$ & $4.5(26)$ & USA \\
\hline 8 & Calories! (8.1.6) & Android & $1-5$ & $4.3(10)$ & Germany \\
\hline 9 & The Secret of Weight (2.4.24) & Android, IOS & $1-5$ & $4.3(14)$ & France \\
\hline 10 & Virtuagym Food (2.4.0) & Android, IOS & $1-5$ & $4.5(28)$ & Netherlands \\
\hline 11 & Health Infinity (HI) (2.0.58) & Android & $0.1-0.5$ & $4.2(9)$ & India \\
\hline 12 & Nutracheck (5.0.12) & Android, IOS & $0.1-0.5$ & $4.3(2)$ & UK \\
\hline
\end{tabular}




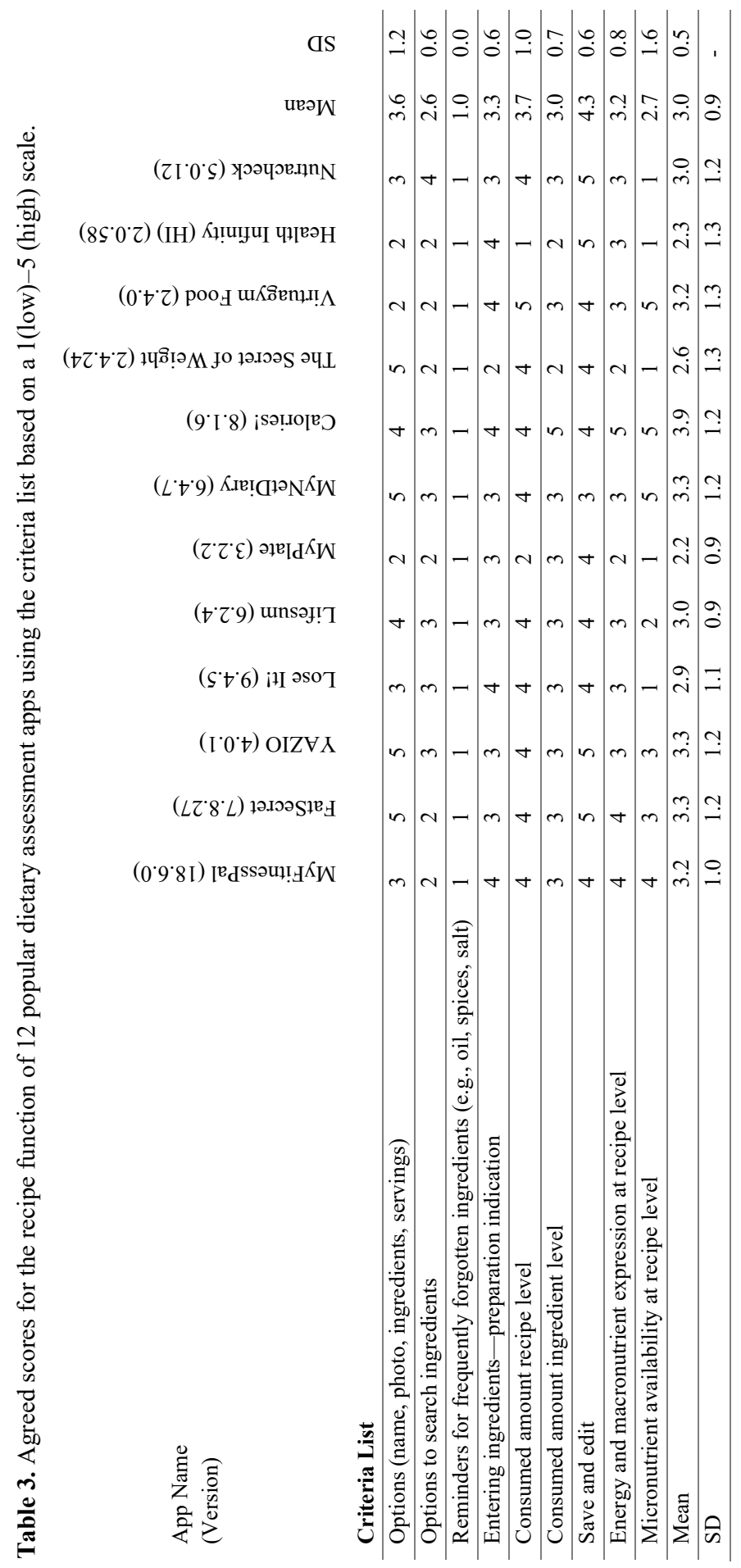




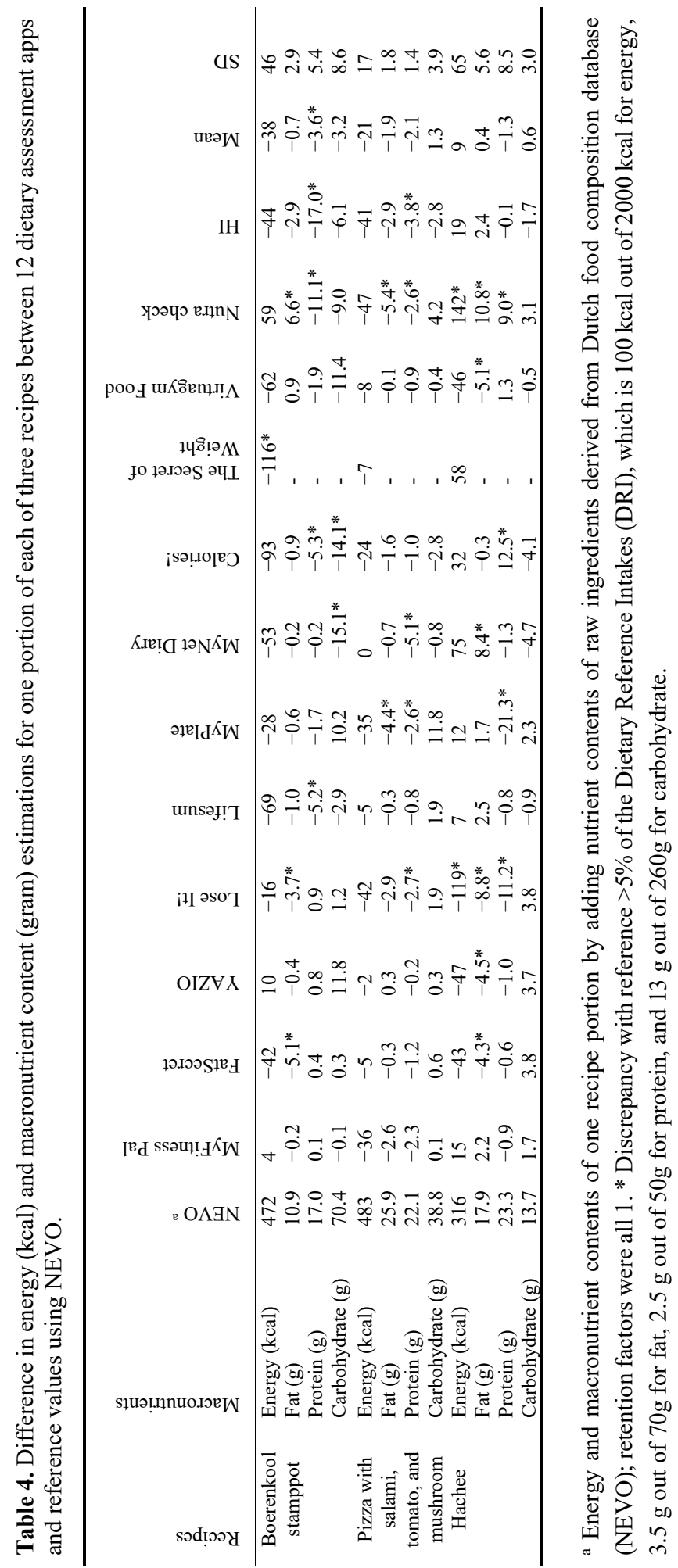




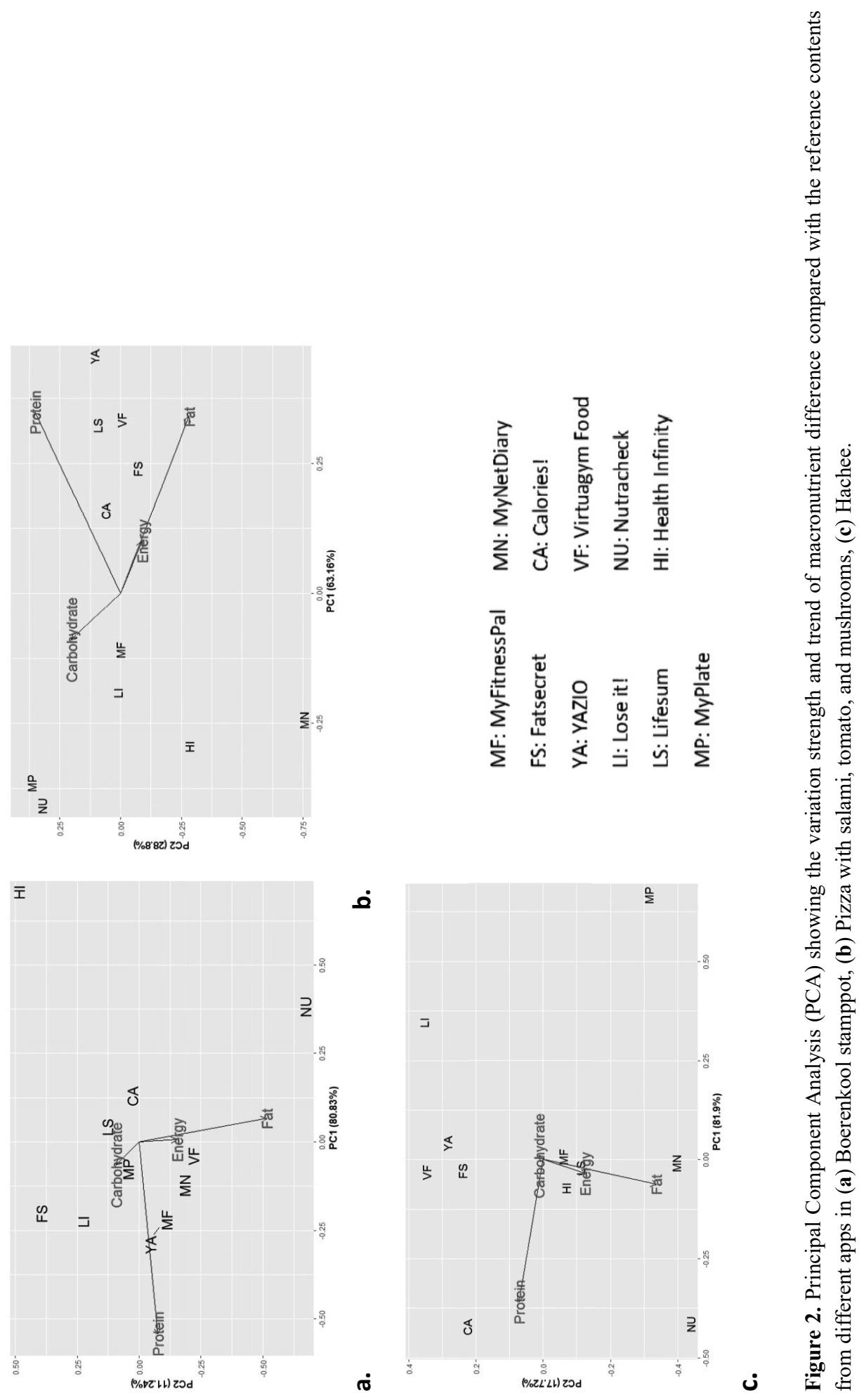




\subsection{Accuracy of micronutrient content estimations}

The micronutrient contents were analyzed for MyNetDiary, Calories! and Virtuagym in which it was available. The differences in micronutrient content estimations of the three recipes between the three popular dietary assessment apps, the micronutrient calculated from NEVO values in raw foods and the reference where retention factors was applied to NEVO are presented in Table 5. For most micronutrients except calcium, applying retention factors resulted in lower micronutrient levels than micronutrient levels in raw ingredients. The relative differences between the reference and using NEVO without applying retention factors ranged from $0 \%$ for calcium in all recipes, vitB12 in stamppot and vitB2 in hachee to more than 45\% for vitamins B6, B12 and folate in hachee. Over the 3 recipes, 8 out of 24 differences $(33 \%)$ were relevant $(>5 \%$ of DRI) in case of a high content and high vulnerability of these nutrients of raw ingredients in a certain recipe. The relatively large difference in vitamin B6 and B12 in Hachee can be explained by the sensitivity to heat and the two cooking procedures in this recipe, i.e. frying and stewing. Whereas, boerenkool stamppot $(n=5)$ had more relevant differences than the other two recipes $(n=1$ and 2 respectively), due to its high contents of vitamin $\mathrm{C}$, vitamin A, vitamin B1, vitamin B6 and folate even if the retention factor was not so different from 1 (for example, vitamin A with a retention factor of 0.9 ).

A larger proportion of difference $>5 \%$ DRI was found in micronutrients (49\%) than in energy and macronutrients (20\%) when compared with the reference values. Among the three apps, MyNetDiary showed more differences $>5 \%$ DRI ( $n=14$ out of 24$)$ than the other two apps (Virtuagym $n=10$, Calories! $n=11$ ) when comparing micronutrient values with the reference. In contrast to macronutrient comparisons, apps more often overestimated the contents of micronutrient in the recipes. The number and extent of overestimations were slightly larger when comparing with the reference than comparing with NEVO without applying retention factors, since the retention factors resulted in lower micronutrient contents in the reference values. The proportions of relevant differences found after comparing the apps to NEVO with or without applying retention factors were rather similar (49\% vs 51\%), illustrating that in many cases the effects of differences in nutrient databases were much larger than differences due to applying retention factors. 


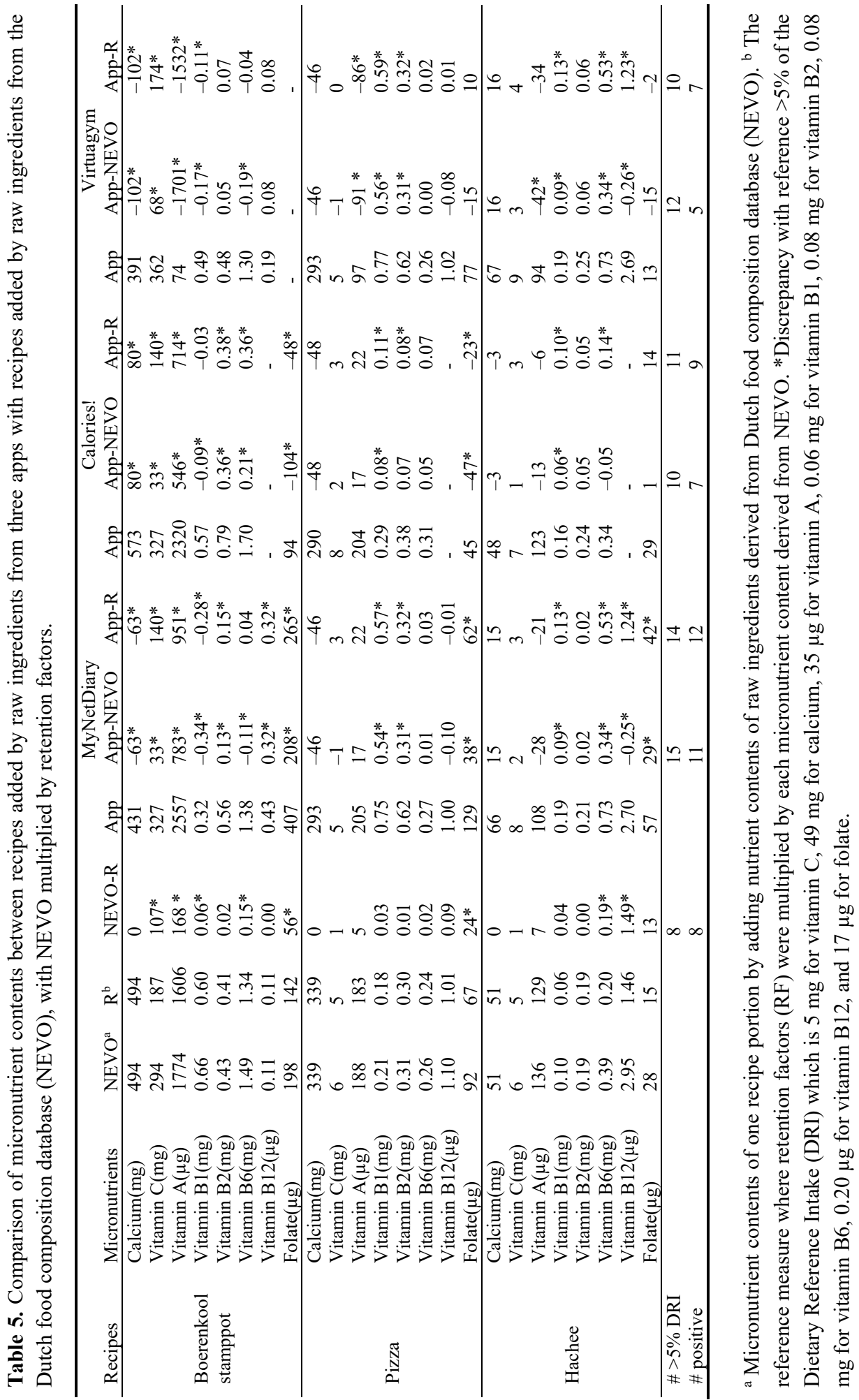




\section{Discussion}

The current study evaluated the recipe function that was available in only one-fifth of the popular available food diary apps. We found a varying quality of recipe features across selected apps which were, on average, judged as suboptimal from research perspectives. Furthermore, capturing the true nutrient intake of mixed dishes is a challenge for this innovative dietary assessment method. A comparison of energy, macro-, and micronutrient contents of recipes between apps with a reference standard recipe calculation showed variation in terms of their ability to accurately estimate nutrient contents. In only three apps was micronutrient information available for recipes, and none of these apps included a procedure to take nutrient losses due to recipe processing into account, and the variability in micronutrient content databases was large.

This is the first study to evaluate the recipe function of current popular dietary assessment apps in a standardized way in which the quality assessment was performed using a rubric of assessment which was made prior to the evaluation. The scores of recipe function were discussed by two researchers, which has eliminated mistakes and the bias of scoring. From the quality assessment of the recipe functions, apps were given a mean overall score of 3.0 (out of 5.0) where the highest score was 3.9 and the lowest 2.2. No correlations were found between the scores given in this study and the popularity and user ratings in app stores. This could illustrate that the recipe function was not the main aspect contributing to users' overall app-experiences, or that researchers and users have different needs for dietary apps [9]. Some simplified features might be favored by users since it was observed that the user's time invested for understanding and learning about an app should be small to sustain long-term app usage [30], whereas researchers are more concerned with features that could enable detailed and accurate data collection. This preference gap between the app users and researchers is important to select suitable features to be included in dietary assessment tools for large nutrition monitoring studies.

Although the quality of recipe function in popular apps was not investigated before [13], several features of a recipe function were investigated by others since they are also relevant for recording food intake. In terms of options for searching ingredients in apps from the current study, all apps had a text searching option and the majority of the apps had a barcode function. Barcode scanning has been shown to save time and was favored by users in recording branded food items, however, the resulting nutrient intake estimation depends largely on the quality of the underlying food composition database within the app [31]. An aspect in which these apps differ from many web-based tools is that most of them do not have portion images, which may due to limited space in the user interface. Previous research has found that the incorporation of portion images was preferred by all age groups [9]. However the overall advantage of using portion images remains unknown [17]. In terms of nutrient information, the energy and macronutrient information was more complete in apps 
than micronutrient information, and this complied with the fact that energy and macronutrients were more closely correlated with weight change, which was the aim for most apps.

Features specific for creating recipes were evaluated. For instance, in addition to other basic features for entering recipes (i.e., add a name, ingredients, and serving number of the recipe), half of the evaluated apps had the capability to enter a photo and cooking explanation. However, this information was not used by the app to estimate nutrient intake. A photo of the recipe could help identify and estimate the amount of food consumed by participants, and could also reduce the extent of underreporting, especially for people with low literacy levels [17], while a cooking explanation provided information relevant for nutrient retention estimation. However, with the extra efforts required in using these features, they might be practical only in small-scale studies. Unlike computer/web-based dietary assessment tools for research purposes [32], all apps lack reminders for frequently forgotten ingredients when creating recipes (e.g., oil, spices, sugar, etc.), which may have partly contributed to the systematic underestimation of macronutrients in most apps found in other studies [33]. Also, current apps did not have pre-defined recipes that could be adapted by users whereas, in some computer-based software, standard recipes could be adapted by switching ingredients or changing the amount of ingredients [32]. However, the practicality of above features to be included in apps or to be used by participants, without the help of researchers, remains questionable. As a simpler alternative, the feature for saving frequently consumed or favorite foods in current apps was shown to save the efforts of users from entering the same recipes repeatedly and searching for food in a comprehensive food list [34].

In the present study, differences in energy, macro-, and micronutrient contents were found between the apps and the reference measure, which could be explained by several reasons. There were substantial differences in the nutrient contents of the recipe ingredients between apps, showing the differences in underlying nutrient databases. Apps were made by companies from different countries and they might have incorporated a nutrient database from their own countries which might have varying nutrient contents for certain foods, due to different cultivating environments [35]. Another source of nutrient values might be input from the app users. This has the benefit of customization of food consumed, however, has shortcomings in the accuracy of nutrients and can lead to quality losses in the food database [14].

The inability to enter exactly the same ingredients across the apps and the limited choice of food amounts may additionally explain part of the variation in nutrient estimation [33]. For example, it was difficult to find an exact match of beef steak in hachee, since there was a large variety of beef steak in different apps, and food amounts in grams were not available in some apps. However, for most other recipe ingredients, this problem did not occur. For 
micronutrients, the difference was also due to applying retention factors to the reference nutrient values, whereas all apps came up with the nutrient content of recipes by simply adding up the nutrient content of each ingredient without taking nutrient retention into account.

Variations of nutrient content of three recipes between apps and the reference measure were observed in the present study, with fewer variations in energy and macronutrient than in micronutrient contents. Similarly, comparable energy contents across apps were also observed in a study where nutrient contents from the barcode scanning of 100 food products in apps were compared with product labels [31]. Likewise, Griffiths et al. compared the results of five commercial apps with thirty $24 \mathrm{~h}$ dietary recalls collected using the Nutrition Data System for Research (NDSR), and found a better validity of energy estimation than nutrients [33]. The mean difference of $22 \mathrm{kcal}$ in energy across all apps and recipes in this study was similar with the $30 \mathrm{kcal}$ mean energy difference of 23 apps compared with the three days' weighed food record in the study of Chen et al. [14]. The wider range of energy difference ( -167 to $262 \mathrm{kcal}$ ) in Chen's study compared to the energy difference in our study $(-118$ to $141 \mathrm{kcal})$ is possibly due to a higher number of apps evaluated, and a larger amount of foods being entered in apps in Chen's study. These findings indicated a relatively reliable energy estimation for both generic and branded food items in the current apps. Still, it was noteworthy that the largest difference of around $345 \mathrm{kcal}$ between apps from both studies could impact the accuracy on both individual and population nutrient intake estimations. A trend of underestimation of energy and macronutrient contents in apps compared to reference in our study was consistent with the study by Griffiths et al. The reason in the study of Griffiths was because the food preparation details were captured by the reference (NDSR), but not in the apps. By contrast, in our study, the food details were equally captured by both the reference and apps, and the reporting bias by participants did not exist since the foods were being entered by researchers. Hence, the main reason of underestimation is the inaccuracy of the nutrition databases within the apps.

A proper way of calculating the nutrient contents within a recipe requires the consideration of nutrient loss during cooking. Currently, the nutrient retention for foods based on different cooking processes is not calculated automatically in any dietary assessment tools, and none of the apps had instructions on using the recipe function. Although existing recipes in food composition tables take the nutrient loss into account, none of the food composition databases cover all the variations on recipes made individually [14]. Alternatively, cooked ingredients could be chosen from the food list. However, the availability of cooked ingredients was incomplete, and this would also require participants to know the amount of the prepared ingredients (which might be smaller due to shrinkage during preparation). Hence, we entered ingredients as raw ingredients, as that is the most logical option for a user. 
This is the first study to investigate the discrepancies of nutrient content between raw ingredients in different apps, compared to a more accurate estimation that takes the nutrient loss into account. Only three out of twelve apps had comprehensive micronutrient information, with both actual amounts and percentage of RDA. The large variation in micronutrient content found in this study implied the importance of choosing the right nutrient database, especially when micronutrient intake estimation is part of the study purposes. The input of raw ingredients potentially leads to overestimation of several heatsensitive micronutrients, which was shown in the micronutrient comparison between NEVO with the reference method in this study. Moreover, the results showed that the extent of difference depends largely on the nutrient contents in the recipe. Therefore, it was suggested that retention factors are most influential when applied to recipes with high micronutrient contents (e.g., boerenkool stamppot).

NEVO was chosen as the reference measure for nutrient estimations, which was a wellmaintained food composition database that had all the data on the nutrition values that were assessed and has a standardized food-compiling procedure that follows the guidelines set by EuroFIR [36,37]. Retention factors applied in this study were the most up-to-date values from the harmonization of retention factors provided by 17 EuroFIR partners [38]. However, the results of nutrient differences may lack representativeness in this study, due to a limited recipe selection. To develop a full picture of the importance of recipe calculation, additional studies, that include more recipes and an evaluation on their contribution to population nutrient intake, will be needed. Furthermore, the evaluation was done only from a research perspective in this study, while user perspective was not analyzed for the apps. Especially factors that could affect the individual's ability to accurately enter the recipe consumed were not examined. Further development of an app for large nutrition monitoring studies would benefit from an evaluation on app users' perspectives.

\section{Conclusion}

In popular food diary apps, the quality of recipe functions is suboptimal from a research perspective. All apps follow a basic nutrition-calculating algorithm, without taking nutrient retention into consideration. This leads to inaccurate nutrient intake estimations in the case that recipes are an important source of micronutrients which are vulnerable to the effects of food processing. Moreover, across apps, there is large variability in nutrient databases. From a research perspective and out of interest regarding micronutrient intake, a balance between user-friendliness and completeness of the recipe function is important. In order to obtain more insight into the need for more complex recipe functionalities, further studies on their potential impact on the nutrient intake estimations in large nutrition-monitoring studies and users' perspective are needed. 


\section{References}

1. Cade, J.E. Measuring Diet in the 21st Century: Use of New Technologies. P Nutr Soc 2017, 76, 276-282.

2. Shim, J.-S.; Oh, K.; Kim, H.C. Dietary Assessment Methods in Epidemiologic Studies. Epidemiology and Health 2014, 36, e2014009.

3. Klesges, R.C.; Eck, L.H.; Ray, J.W. Who Underreports Dietary Intake in a Dietary Recall? Evidence from the Second National Health and Nutrition Examination Survey. Journal of consulting and clinical psychology 1995, 63, 438-444.

4. Livingstone, M.B.; Robson, P.J.; Wallace, J.M. Issues in Dietary Intake Assessment of Children and Adolescents. The British journal of nutrition 2004, 92 Suppl 2, S213222.

5. Schober, M.F. Asking Questions and Influencing Answers. Questions about questions: Inquiries into the cognitive bases of surveys 1992, 15.

6. Boushey, C.J.; Spoden, M.; Zhu, F.M.; Delp, E.J.; Kerr, D.A. New Mobile Methods for Dietary Assessment: Review of Image-Assisted and Image-Based Dietary Assessment Methods. The Proceedings of the Nutrition Society 2017, 76, 283-294.

7. Ramachandran, K. Nutrition Monitoring and Surveillance. NFI BULLETIN 2006, 27, 5.

8. Ocke, M.C.; Van Rossum, C.T.M.; de Boer, E.J. Dietary Surveys: National Food Intake. In The Encyclopedia of Food and Health, Caballero, B.; Finglas, P.; Toldrà, F., Eds. Academic Press: Oxford, 2016; Vol. 2, pp 432-438.

9. Carter, M.C.; Albar, S.A.; Morris, M.A.; Mulla, U.Z.; Hancock, N.; Evans, C.E.; Alwan, N.A.; Greenwood, D.C.; Hardie, L.J.; Frost, G.S., et al. Development of a UK Online 24-H Dietary Assessment Tool: Myfood24. Nutrients 2015, 7, 4016-4032.

10. Boshuizen, H.C.; Viet, A.L.; Picavet, H.S.; Botterweck, A.; van Loon, A.J. NonResponse in a Survey of Cardiovascular Risk Factors in the Dutch Population: Determinants and Resulting Biases. Public health 2006, 120, 297-308.

11. Conrad, J.; Nothlings, U. Innovative Approaches to Estimate Individual Usual Dietary Intake in Large-Scale Epidemiological Studies. P Nutr Soc 2017, 76, 213-219.

12. Carter, M.C.; Burley, V.J.; Nykjaer, C.; Cade, J.E. 'My Meal Mate' (Mmm): Validation of the Diet Measures Captured on a Smartphone Application to Facilitate Weight Loss. The British journal of nutrition 2013, 109, 539-546.

13. Xu, W.; Liu, Y. Mhealthapps: A Repository and Database of Mobile Health Apps. JMIR Mhealth Uhealth 2015, 3, e28.

14. Chen, J.; Cade, J.E.; Allman-Farinelli, M. The Most Popular Smartphone Apps for Weight Loss: A Quality Assessment. JMIR Mhealth Uhealth 2015, 3, e104.

15. Aguilar-Martinez, A.; Sole-Sedeno, J.M.; Mancebo-Moreno, G.; Medina, F.X.; Carreras-Collado, R.; Saigi-Rubio, F. Use of Mobile Phones as a Tool for Weight Loss: A Systematic Review. Journal of Telemedicine and Telecare 2014, 20, 339-349. 
16. Dennison, L.; Morrison, L.; Conway, G.; Yardley, L. Opportunities and Challenges for Smartphone Applications in Supporting Health Behavior Change: Qualitative Study. J Med Internet Res 2013, 15, e86.

17. Lieffers, J.R.L.; Hanning, R.M. Dietary Assessment and Self-Monitoring: With Nutrition Applications for Mobile Devices. Canadian Journal of Dietetic Practice and Research 2012, 73, e253-e260.

18. Dietary Assessment: A Resource Guide to Method Selection and Application in Low Resource Settings.; FAO: Rome, 2018.

19. Illner, A.K.; Freisling, H.; Boeing, H.; Huybrechts, I.; Crispim, S.P.; Slimani, N. Review and Evaluation of Innovative Technologies for Measuring Diet in Nutritional Epidemiology. Int J Epidemiol 2012, 41, 1187-1203.

20. Reinivuo, H.; Bell, S.; Ovaskainen, M.-L. Harmonisation of Recipe Calculation Procedures in European Food Composition Databases. J Food Compos Anal 2009, 22, 410-413.

21. Maringer, M.; van’t Veer, P.; Klepacz, N.; Verain, M.C.D.; Normann, A.; Ekman, S.; Timotijevic, L.; Raats, M.M.; Geelen, A. User-Documented Food Consumption Data from Publicly Available Apps: An Analysis of Opportunities and Challenges for Nutrition Research. Nutrition Journal 2018, 17, 59.

22. Franco, Z.R.; Fallaize, R.; Lovegrove, A.J.; Hwang, F. Popular Nutrition-Related Mobile Apps: A Feature Assessment. JMIR Mhealth Uhealth 2016, 4, e85.

23. Severi, S.; Bedogni, G.; Zoboli, G.P.; Manzieri, A.M.; Poli, M.; Gatti, G.; Battistini, N. Effects of Home-Based Food Preparation Practices on the Micronutrient Content of Foods. European journal of cancer prevention : the official journal of the European Cancer Prevention Organisation 1998, 7, 331-335.

24. Slimani, N.; Deharveng, G.; Charrondiere, R.U.; van Kappel, A.L.; Ocke, M.C.; Welch, A.; Lagiou, A.; van Liere, M.; Agudo, A.; Pala, V., et al. Structure of the Standardized Computerized 24-H Diet Recall Interview Used as Reference Method in the 22 Centers Participating in the Epic Project. European Prospective Investigation into Cancer and Nutrition. Computer methods and programs in biomedicine 1999, 58, 251-266.

25. van Rossum, C.T.M.; Fransen, H.P.; Verkaik-Kloosterman, J.; Buurma, E.M.; Ocke, M.C. Dutch National Food Consumption Survey 2007-2010: Diet of Children and Adults Aged 7 to 69 Years; RIVM-report 350070006; RIVM: Bilthoven, 2011.

26. RIVM. Nevo-Table. Available online: https://nevo-online.rivm.nl/ (accessed on 23 July 2018),

27. Bognár, A. Tables on Weight Yield of Food and Retention Factors of Food Constituents for the Calculation of Nutrient Composition of Cooked Foods (Dishes). BFE Karlsruhe: Karlsruhe, Germany, 2002.

28. Reference Intake. Available online: https://www.voedingscentrum.nl/encyclopedie/referentie-inname.aspx (accessed on 26 December 2018), 
29. Barr, S.I.; Murphy, S.P.; Poos, M.I. Interpreting and Using the Dietary References Intakes in Dietary Assessment of Individuals and Groups. Journal of the American Dietetic Association 2002, 102, 780-788.

30. Spriestersbach, A.; Springer, T. Quality Attributes in Mobile Web Application Development. Lect Notes Comput Sc 2004, 3009, 120-130.

31. Maringer, M.; Wisse-Voorwinden, N.; Veer, P.V.; Geelen, A. Food Identification by Barcode Scanning in the Netherlands: A Quality Assessment of Labelled Food Product Databases Underlying Popular Nutrition Applications. Public health nutrition 2018, 1-8.

32. Slimani, N.; Casagrande, C.; Nicolas, G.; Freisling, H.; Huybrechts, I.; Ocke, M.C.; Niekerk, E.M.; van Rossum, C.; Bellemans, M.; De Maeyer, M., et al. The Standardized Computerized 24-H Dietary Recall Method Epic-Soft Adapted for PanEuropean Dietary Monitoring. Eur J Clin Nutr 2011, 65 Suppl 1, S5-15.

33. Griffiths, C.; Harnack, L.; Pereira, M.A. Assessment of the Accuracy of Nutrient Calculations of Five Popular Nutrition Tracking Applications. Public health nutrition 2018, 21, 1495-1502.

34. Rusin, M.; Årsand, E.; Hartvigsen, G. Functionalities and Input Methods for Recording Food Intake: A Systematic Review. International journal of medical informatics 2013, 82, 653-664.

35. Marconi, S.; Durazzo, A.; Camilli, E.; Lisciani, S.; Gabrielli, P.; Aguzzi, A.; Gambelli, L.; Lucarini, M.; Marletta, L. Food Composition Databases: Considerations About Complex Food Matrices. Foods 2018, 7.

36. Castanheira, I.; Roe, M.; Westenbrink, S.; Ireland, J.; Møller, A.; Salvini, S.; Beernaert, H.; Oseredczuk, M.; Calhau, M.A. Establishing Quality Management Systems for European Food Composition Databases. Food chemistry 2009, 113, 776780 .

37. Westenbrink, S.; Oseredczuk, M.; Castanheira, I.; Roe, M. Food Composition Databases: The Eurofir Approach to Develop Tools to Assure the Quality of the Data Compilation Process. Food chemistry 2009, 113, 759-767.

38. Vasquez-Caicedo, A.L.; Bell, S.; Hartmann, B. Report on Collection of Rules on Use of Recipe Calculation Procedures Including the Use of Yield and Retention Factors for Imputing Nutrient Values for Composite Foods; European Food Information Resource: Brussels, Belgium, 2014. 
Table S1: Energy and macronutrient differences between app and NEVO for stamppot

\begin{tabular}{|c|c|c|c|c|c|c|c|c|}
\hline & $\begin{array}{c}\text { Fat } \\
\text { (g) }\end{array}$ & $\begin{array}{c}\text { Protei } \\
\text { n (g) }\end{array}$ & $\begin{array}{c}\text { Carbohydrat } \\
\text { e (g) }\end{array}$ & \begin{tabular}{|c|} 
Energ \\
$\mathbf{y}$ \\
$(\mathrm{kcal})$
\end{tabular} & \begin{tabular}{|c|} 
Energy \\
differenc \\
e with \\
reference \\
\end{tabular} & \begin{tabular}{|c|} 
Fat \\
differenc \\
e with \\
reference
\end{tabular} & \begin{tabular}{|c|} 
Protein \\
differenc \\
e with \\
reference
\end{tabular} & $\begin{array}{l}\text { Carbohydrat } \\
\text { e difference } \\
\text { with } \\
\text { reference } \\
\end{array}$ \\
\hline \begin{tabular}{|l|} 
Reference \\
raw
\end{tabular} & 10.9 & 16.95 & 70.375 & 472.25 & 0 & 0 & 0 & 0 \\
\hline \begin{tabular}{|l|} 
Myfitnesspa \\
1
\end{tabular} & 10.7 & 17 & 70.3 & 476 & 3.75 & -0.2 & 0.05 & -0.075 \\
\hline Fatsecret & 5.78 & 17.39 & 70.66 & 430 & -42.25 & -5.12 & 0.44 & 0.285 \\
\hline YAZIO & 10.5 & 17.7 & 82.2 & 482 & 9.75 & -0.4 & 0.75 & 11.825 \\
\hline Lose it! & 7.2 & 17.8 & 71.6 & 456 & -16.25 & -3.7 & 0.85 & 1.225 \\
\hline Lifesum & 9.9 & 11.8 & 67.5 & 403 & -69.25 & -1 & -5.15 & -2.875 \\
\hline MyPlate & $\begin{array}{c}10.3 \\
3\end{array}$ & 15.26 & 80.53 & 444 & -28.25 & -0.57 & -1.69 & 10.155 \\
\hline MyNetDiary & $\begin{array}{c}10.7 \\
5\end{array}$ & 16.75 & 55.25 & 419 & -53.25 & -0.15 & -0.2 & -15.125 \\
\hline Calories! & 10 & 11.7 & 56.3 & \begin{tabular}{|l|}
379.6 \\
\end{tabular} & -92.65 & -0.9 & -5.25 & -14.075 \\
\hline $\begin{array}{l}\text { The Secret } \\
\text { of Weight }\end{array}$ & - & - & - & 356.25 & -116 & - & - & - \\
\hline $\begin{array}{l}\text { Virtuagym } \\
\text { Food }\end{array}$ & 11.8 & 15.03 & 59.02 & 410 & -62.25 & 0.9 & -1.92 & -11.355 \\
\hline Nutracheck & 17.5 & 5.9 & 61.4 & 531 & 58.75 & 6.6 & -11.05 & -8.975 \\
\hline $\mathrm{HI}$ & 8 & 0 & 64.3 & 428.1 & -44.15 & -2.9 & -16.95 & -6.075 \\
\hline
\end{tabular}


Table S2: Energy and macronutrient differences between app and NEVO for pizza salami, tomato, mushroom

\begin{tabular}{|l|c|c|c|c|c|c|c|c|}
\hline & $\begin{array}{c}\text { Fat } \\
\mathbf{( g )}\end{array}$ & $\begin{array}{c}\text { Protei } \\
\mathbf{n}(\mathbf{g})\end{array}$ & $\begin{array}{c}\text { Carbohydrat } \\
\mathbf{e}(\mathbf{g})\end{array}$ & $\begin{array}{c}\text { Energ } \\
\mathbf{y} \\
\mathbf{( k c a l )}\end{array}$ & $\begin{array}{c}\text { Energy } \\
\text { differenc } \\
\mathbf{e} \text { with } \\
\text { reference }\end{array}$ & $\begin{array}{c}\text { Fat } \\
\text { differenc } \\
\mathbf{e} \text { wit } \\
\text { reference }\end{array}$ & $\begin{array}{c}\text { Protein } \\
\text { differenc } \\
\text { e with } \\
\text { reference }\end{array}$ & $\begin{array}{c}\text { Carbohydrat } \\
\text { e difference } \\
\text { with } \\
\text { reference }\end{array}$ \\
\hline $\begin{array}{l}\text { Reference } \\
\text { raw }\end{array}$ & $\begin{array}{c}25.92 \\
5\end{array}$ & 22.075 & 38.8 & 482.75 & 0 & 0 & 0 & 0 \\
\hline $\begin{array}{l}\text { Myfitnesspa } \\
\text { l }\end{array}$ & 23.3 & 19.8 & 38.9 & 447 & -35.75 & -2.625 & -2.275 & 0.1 \\
\hline Fatsecret & 25.64 & 20.87 & 39.41 & 478 & -4.75 & -0.285 & -1.205 & 0.61 \\
\hline YAZIO & 26.2 & 21.9 & 39.1 & 481 & -1.75 & 0.275 & -0.175 & 0.3 \\
\hline Lose it! & 23 & 19.4 & 40.7 & 440.4 & -42.35 & -2.925 & -2.675 & 1.9 \\
\hline Lifesum & 25.6 & 21.3 & 40.7 & 478 & -4.75 & -0.325 & -0.775 & 1.9 \\
\hline MyPlate & 21.48 & 19.49 & 50.63 & 448 & -34.75 & -4.445 & -2.585 & 11.83 \\
\hline $\begin{array}{l}\text { MyNetDiar } \\
\text { y }\end{array}$ & 25.25 & 17 & 38 & 482.75 & 0 & -0.675 & -5.075 & -0.8 \\
\hline Calories! & 24.3 & 21.1 & 36 & 459.2 & -23.55 & -1.625 & -0.975 & -2.8 \\
\hline $\begin{array}{l}\text { The Secret } \\
\text { of Weight }\end{array}$ & - & - & - & 476 & -6.75 & - & - & - \\
\hline $\begin{array}{l}\text { Virtuagym } \\
\text { Food }\end{array}$ & 25.85 & 21.13 & 38.42 & 475 & -7.75 & -0.075 & -0.945 & -0.38 \\
\hline Nutracheck & 20.5 & 19.5 & 43 & 436 & -46.75 & -5.425 & -2.575 & 4.2 \\
\hline HI & 23 & 18.3 & 36 & 442.1 & -40.65 & -2.925 & -3.775 & -2.8 \\
\hline
\end{tabular}


Table S3: Energy and macronutrient differences between app and NEVO for hachee

\begin{tabular}{|c|c|c|c|c|c|c|c|c|}
\hline & \begin{tabular}{|c} 
Fat \\
(g)
\end{tabular} & $\begin{array}{c}\text { Protei } \\
\text { n (g) }\end{array}$ & $\begin{array}{c}\text { Carbohydrat } \\
\text { e (g) }\end{array}$ & \begin{tabular}{|c|} 
Energ \\
$\mathbf{y}$ \\
$(\mathrm{kcal})$
\end{tabular} & $\begin{array}{c}\text { Energy } \\
\text { differenc } \\
\text { e with } \\
\text { reference }\end{array}$ & \begin{tabular}{|c|} 
Fat \\
differenc \\
e with \\
reference
\end{tabular} & \begin{tabular}{|c|} 
Protein \\
differenc \\
e with \\
reference
\end{tabular} & $\begin{array}{c}\text { Carbohydrat } \\
\text { e difference } \\
\text { with } \\
\text { reference }\end{array}$ \\
\hline $\begin{array}{l}\text { Reference } \\
\text { raw }\end{array}$ & \begin{tabular}{|c|}
17.8 \\
5
\end{tabular} & 23.325 & 13.7 & 315.5 & 0 & 0 & 0 & 0 \\
\hline $\begin{array}{l}\text { Myfitnesspa } \\
1\end{array}$ & 20 & 22.4 & 15.4 & 330 & 14.5 & 2.15 & -0.925 & 1.7 \\
\hline Fatsecret & $\begin{array}{c}13.5 \\
9\end{array}$ & 22.7 & 17.51 & 273 & -42.5 & -4.26 & -0.625 & 3.81 \\
\hline YAZIO & 13.4 & 22.3 & 17.4 & 269 & -46.5 & -4.45 & -1.025 & 3.7 \\
\hline Lose it! & 9.1 & 12.1 & 17.5 & 197 & -118.5 & -8.75 & -11.225 & 3.8 \\
\hline \begin{tabular}{|l|} 
Lifesum \\
\end{tabular} & 20.3 & 22.5 & 12.8 & 322 & 6.5 & 2.45 & -0.825 & -0.9 \\
\hline MyPlate & \begin{tabular}{|c|}
19.5 \\
1 \\
\end{tabular} & 2.05 & 15.95 & 327 & 11.5 & 1.66 & -21.275 & 2.25 \\
\hline MyNetDiary & $\begin{array}{c}26.2 \\
5\end{array}$ & 22 & 9 & 390.25 & 74.75 & 8.4 & -1.325 & -4.7 \\
\hline Calories! & 17.6 & 35.8 & 9.6 & 347.5 & 32 & -0.25 & 12.475 & -4.1 \\
\hline $\begin{array}{l}\text { The Secret } \\
\text { of Weight }\end{array}$ & - & - & - & 373 & 57.5 & - & - & - \\
\hline $\begin{array}{l}\text { Virtuagym } \\
\text { Food }\end{array}$ & $\begin{array}{c}12.7 \\
2\end{array}$ & 24.59 & 13.17 & 270 & -45.5 & -5.13 & 1.265 & -0.53 \\
\hline Nutracheck & 28.6 & 32.3 & 16.8 & 457 & 141.5 & 10.75 & 8.975 & 3.1 \\
\hline $\mathrm{HI}$ & 20.2 & 23.2 & 12 & 334.4 & 18.9 & 2.35 & -0.125 & -1.7 \\
\hline
\end{tabular}




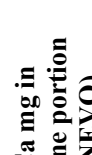

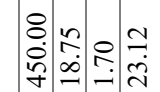

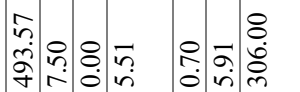

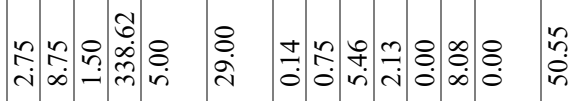

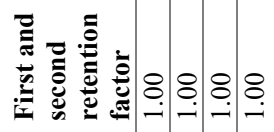

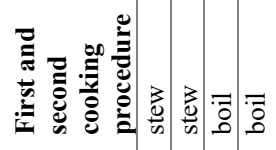

จี

วิว วิ วิว วิ

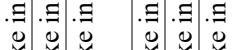

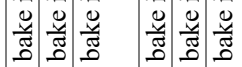

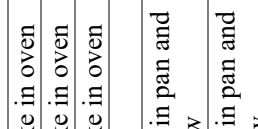

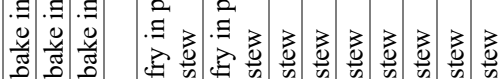

竞

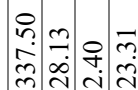

min:

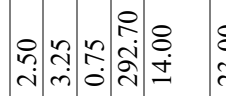

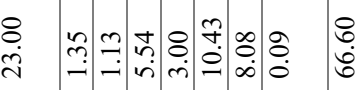

تِ

:

in

inj

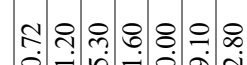

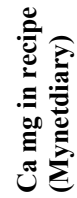

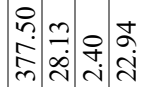

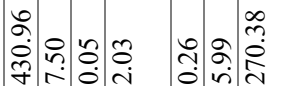

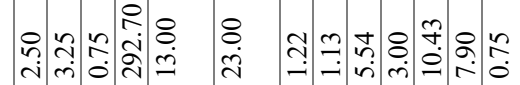

ชุ

שั

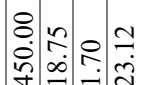

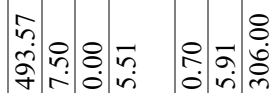

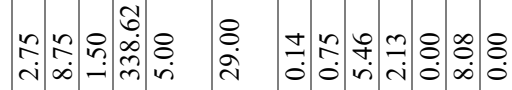

in

河

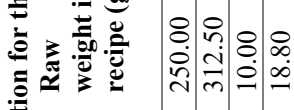

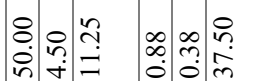

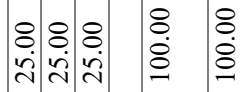

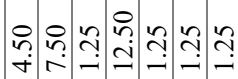

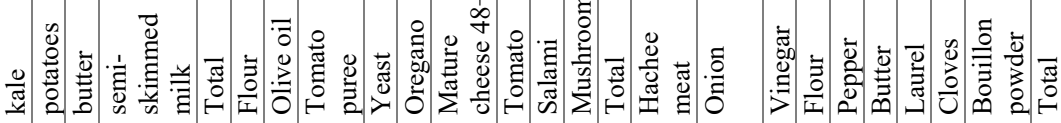

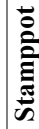

离

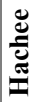




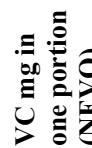

:. 穴:

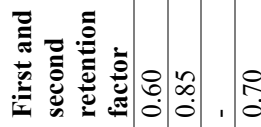

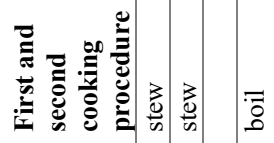

ปี

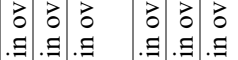

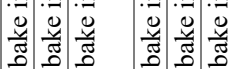

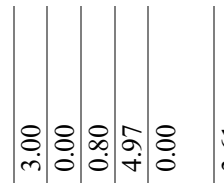

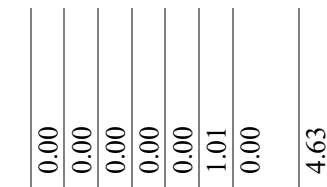

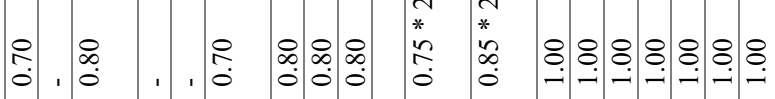

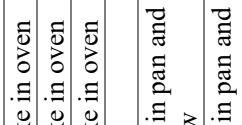

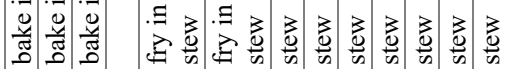

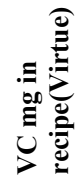

迎

奋:

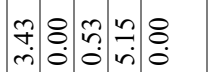

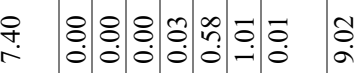

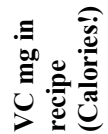

กิ:

ஸें

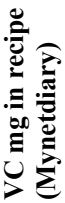

ì

ஸ̂

$\stackrel{\infty}{\curvearrowright}$

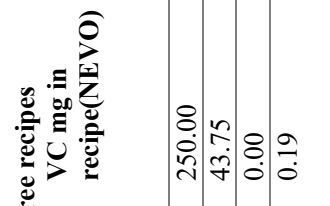

ஸें

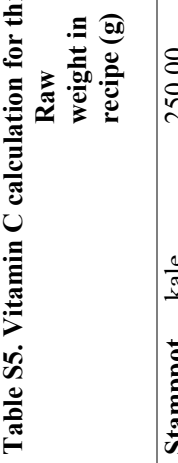

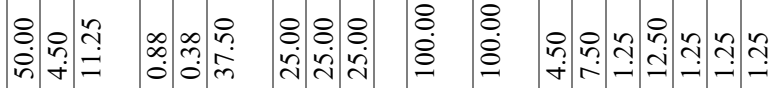

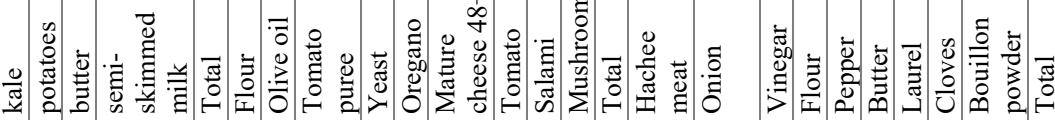

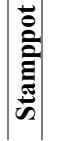

胥

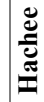




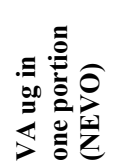

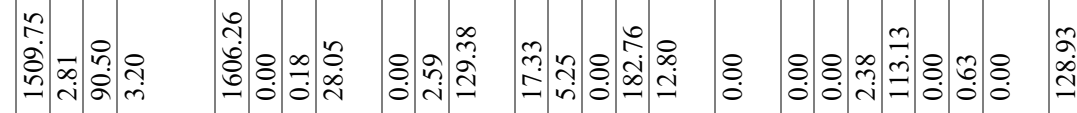

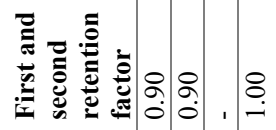

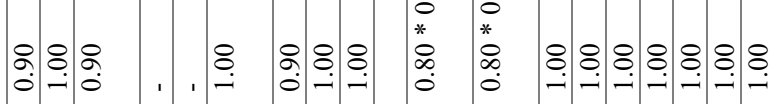

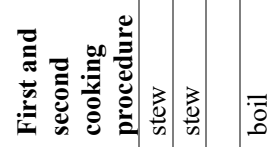

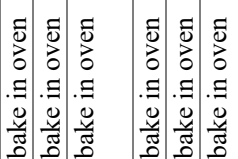

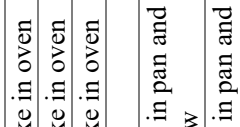

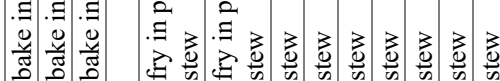

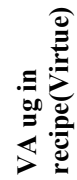

:

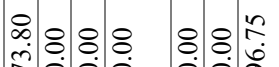

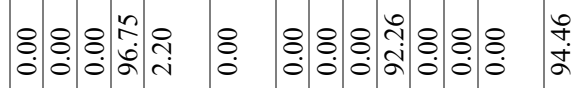

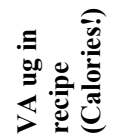

in:

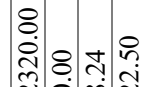

$8: \stackrel{m}{0}: 0$

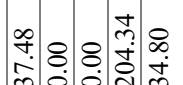

ㄴ.

更

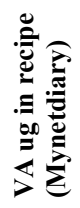

$8 \sqrt{1.5}+\frac{1}{4}$

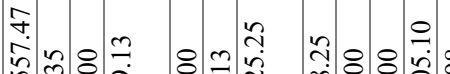

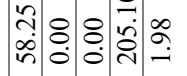

:

논.

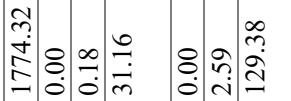

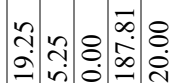

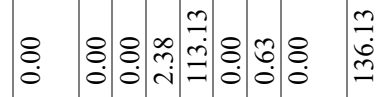

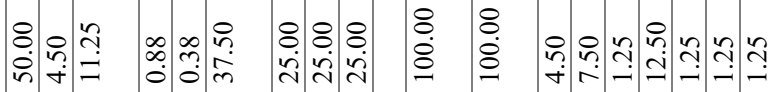

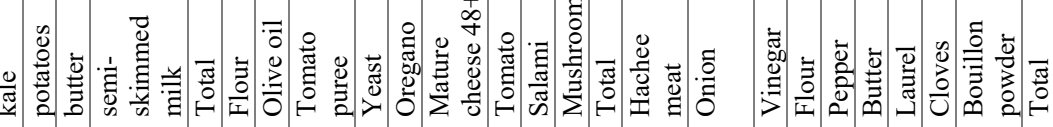

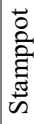

ำ

$\frac{\mathscr{d}}{\stackrel{0}{0}}$ 


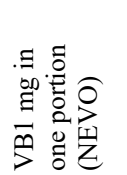

争:

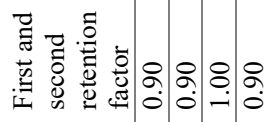

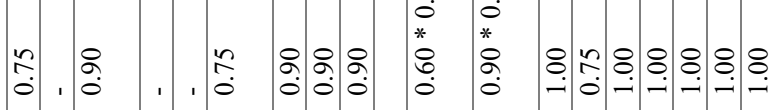

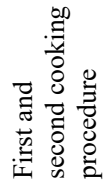

跤

วิว วิด วิว

$\exists . \Xi \quad \exists . \exists . \Xi$

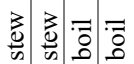

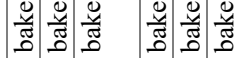

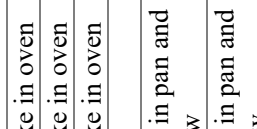

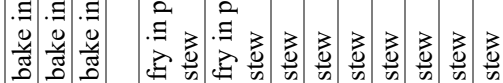

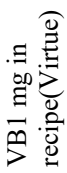

范

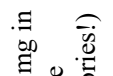

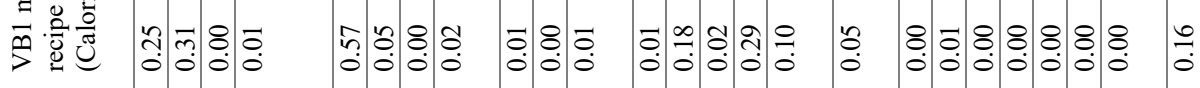

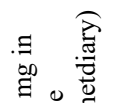

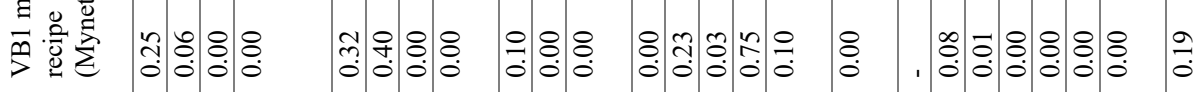

D.

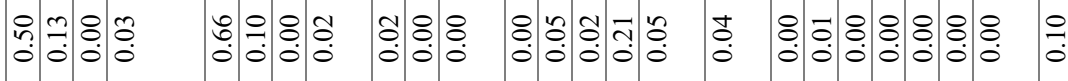

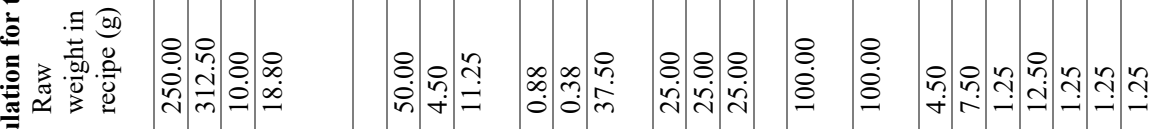

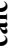

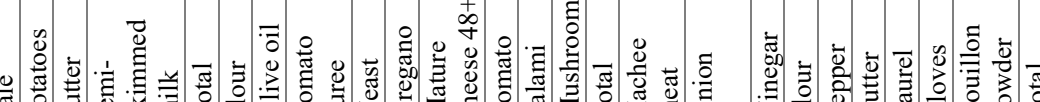

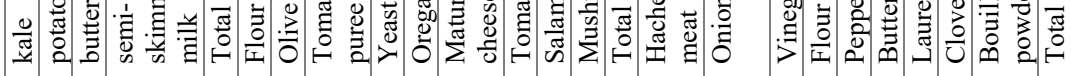

离

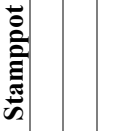

$\stackrel{\mathfrak{3}}{\mathfrak{\Sigma}}$

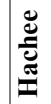




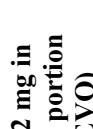

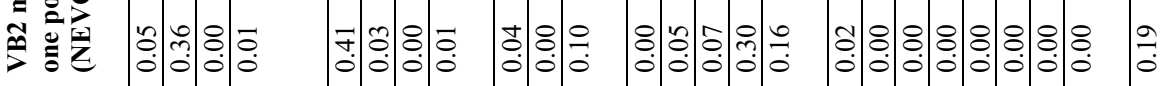

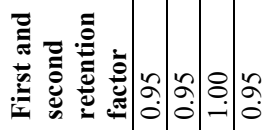

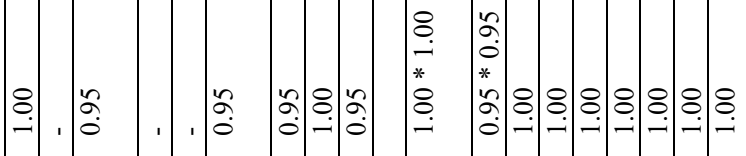

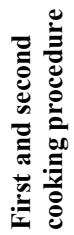

|

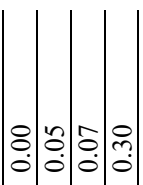

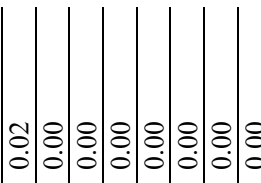

$1 \frac{1}{0}$
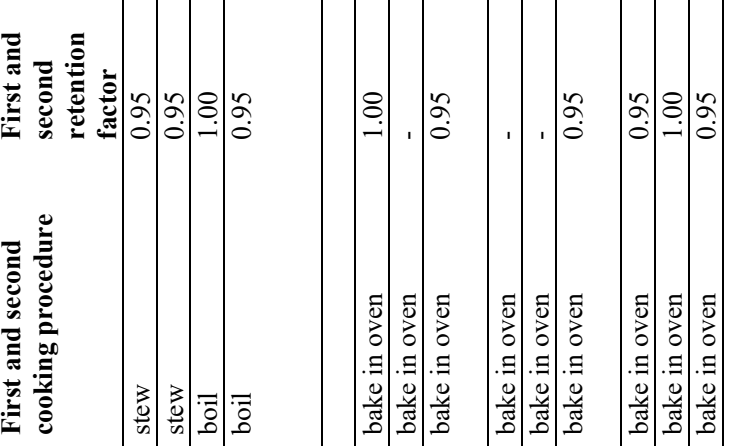

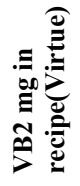

离

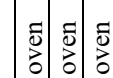

$. \Xi . \Xi . \Xi$

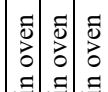

藏

$\because: \Xi . \Xi \quad . \Xi \ldots$

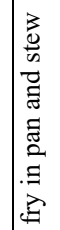

亲

กิ:

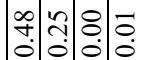

பீ:日:

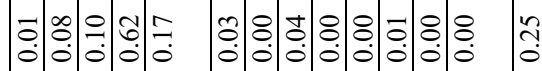

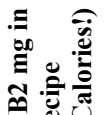

กิ้

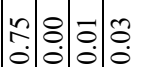

ลิ:

ำำำ

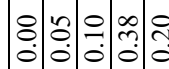

m

范

กิ:

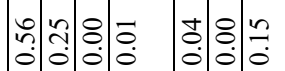

$8: 00$

๑:

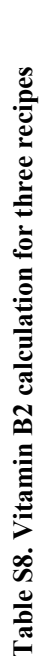

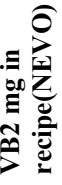

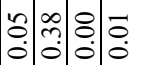

กิ 2.

t)

:

$\Xi 0$

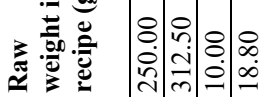

帒

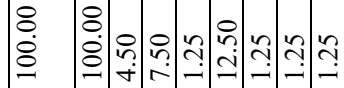

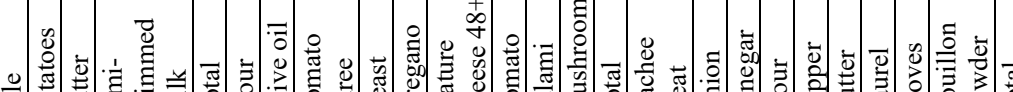

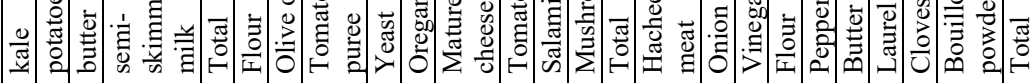

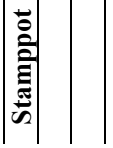

胥

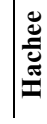




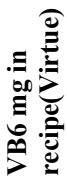

:

ஸे:

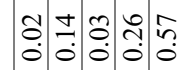

$\frac{1}{0}$

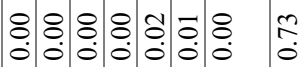

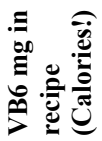

ㄴ.

유:웅유.

:웅:

둥: ํํ:

ㄱ.

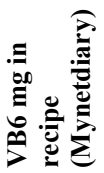

ㄴ.

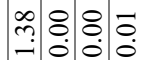

:

:

,

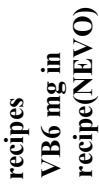

กุ.

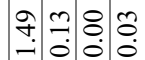

둥:

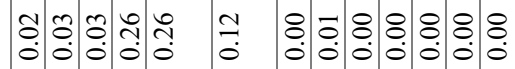

के

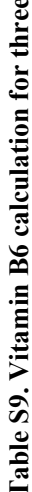

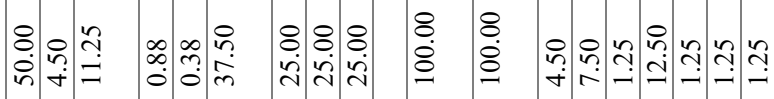

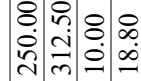

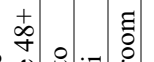

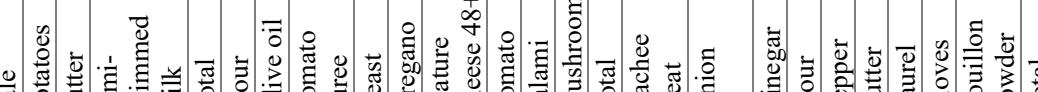

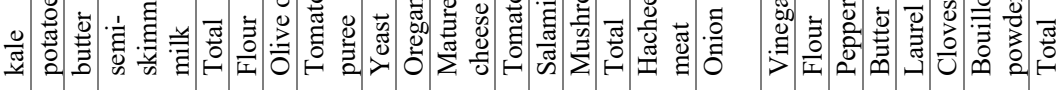

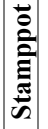

:

冚 


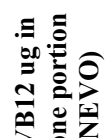

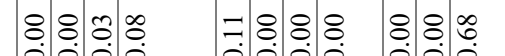

ஜ

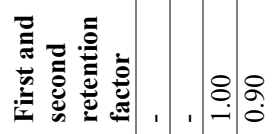

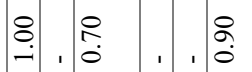

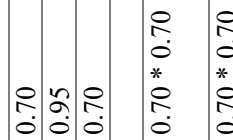

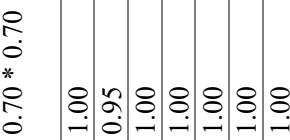

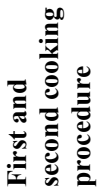

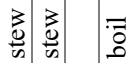

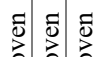

₹ ธ

$\exists . \Xi . \Xi$

응

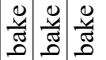

$. \Xi . \Xi$

ธี

เว

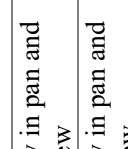

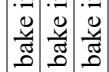

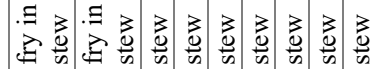

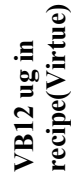

$\because: 0 \frac{0}{0}: \frac{0}{0}$

웅:

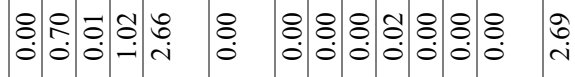

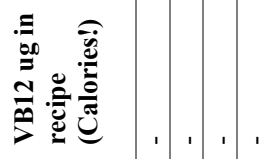

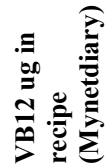

:

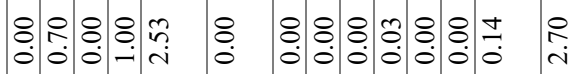

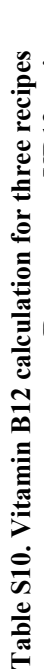

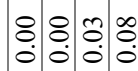

$\exists: \frac{8}{0}: \frac{8}{0}: \frac{n}{0}$

:

: ,

Ð $\equiv$

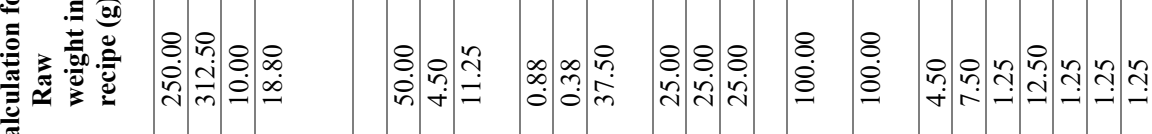

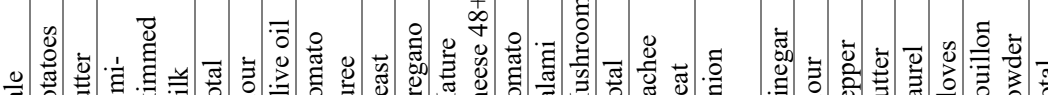

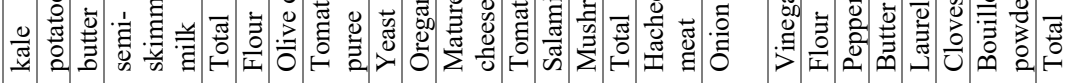

产

胥

$\underset{\Xi}{ \pm}$ 



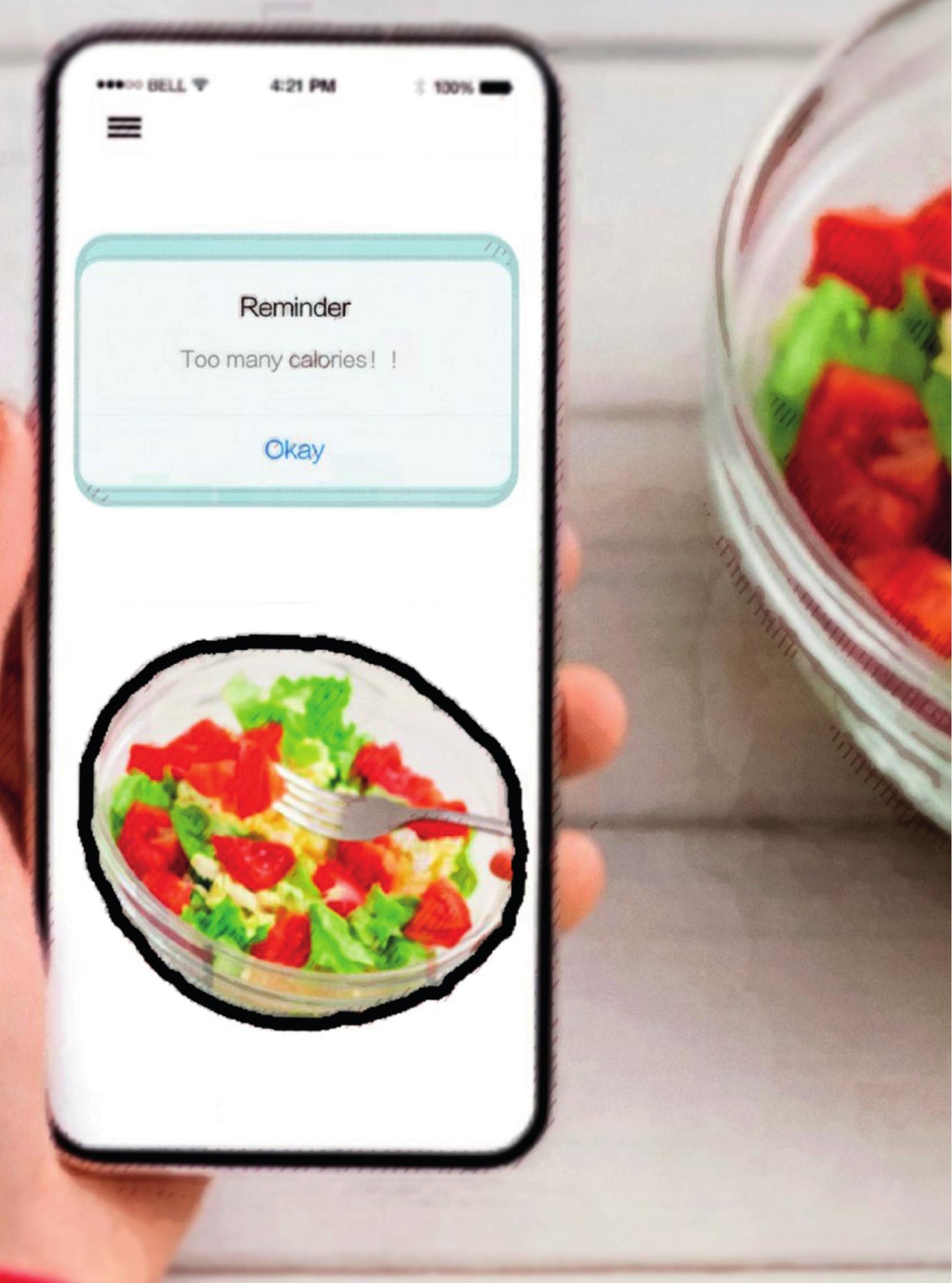




\section{Chapter 5}

\section{A Systematic Review and Meta-analysis of Validation Studies Performed on Food Record Apps}

Liangzi Zhang, Andreja Misir, Hendriek Boshuizen, Marga Ocké Submitted for publication 


\begin{abstract}
Mobile food record apps have been increasingly validated by studies with various study designs. This review aims to evaluate the overall accuracy of FR apps in measuring the intake of energy, macro- and micronutrients, food groups in real-life settings and to provide a summary of the study designs used in these studies. We systematically searched online databases for mobile FR validation studies published during 2013-2019. We identified 14 studies for the systematic review, of which 11 studies were suitable for meta-analyses on energy intake and eight for meta-analysis on macronutrient intake. Mean differences and SDs for each outcome were pooled using a random-effects model. All apps underestimated energy intake when compared to their reference methods with a pooled effect of -202 kcal (-319 to $-85 \mathrm{kcal})$. After stratification, studies which used the same food composition tables for both the app and the reference method had no heterogeneity with a pooled effect of $-57 \mathrm{kcal}(-116$ to $2 \mathrm{kcal}$ ). In eight studies that investigated macronutrient intake, after excluding outliers, the heterogeneity of carbohydrate, fat, and protein was $54 \%, 73 \%$ and $80 \%$, with the pooled effect of $-18.8 \mathrm{~g} /$ day, $-12.7 \mathrm{~g} /$ day, and $-12.2 \mathrm{~g} /$ day respectively. Micronutrients from six studies and food groups from four studies were - mostly statistically insignificantly underestimated by the apps. Alcohol was significantly overestimated by one app while significantly underestimated by another app. This review concluded that FR apps seem to underreport dietary intake slightly more than traditional dietary assessment methods. Better quality validation studies should be conducted in the future, i.e. by applying biomarkers as the reference; testing in larger and more representative study populations for longer periods; avoiding learning effect of each method; comparing food groups and micronutrients with both raw data and adjusted values.
\end{abstract}




\section{Introduction}

Diet has been recognized as one of the determinants for developing non-communicable diseases such as cardiovascular disease, diabetes, and cancer (1). An accurate assessment of dietary intake is fundamental for carrying out nutritional studies (2). Self-reported dietary intake is the most commonly used method in large scale nutritional studies, which could assess all food and nutrients and has a better trade-off between cost, response and accuracy than objective measures (e.g., biomarkers) (3). However, self-reported intake may be subject to response error (inaccurate recall, under- and overreporting) and portion size error (inaccurate portion size assessment) $(4,5)$. Retrospective methods such as 24-hour recall (24HR) are subject to memory loss, while prospective methods such as food records are subject to reactivity bias (6), but are better in estimating portion sizes (7).

Due to the error-prone nature and burdensome procedures in available dietary assessment methods, technology advancement has favored the use of digital applications in assessing dietary intake in large-scale studies (8-10). Most interesting is mobile phone ownership that has grown exponentially in the past two decades, providing a convenient platform for recording dietary intake (11). Specifically, mobile applications were constructed based on the theory of traditional dietary assessment methods are among the main instruments investigated in nutritional studies nowadays (11). Most mobile dietary apps have an underlying mechanism of food records, due to the portable nature of smartphones, and the ability to incorporate real-time recording features like barcode and photo recognition to assist in food searching and portion size estimation (12).

New methods (and technologies) need to be validated to ensure accuracy in estimating dietary intake before being applied in large-scale research. Validation studies assess the degree to which a new method measures what it is intending to measure by comparing with a reference method (13). The reference method should have a higher degree of demonstrated validity and have uncorrelated errors with the test method (14). Currently, most researchbased apps have been validated with a well-established dietary assessment method, while only few commercial apps have been validated (8).

The quality of existing validation studies depends on the resources and methodologies that researchers can access (8). There are no recent reviews on the results of validation studies that specifically focused on food record apps. A review study by Sharp et al. focused on evaluating the validity, feasibility, and acceptability of a broader range of technologies, including both dietary apps and image-based technologies. They concluded that these technologies showed similar, but not superior validity when compared with conventional methods (9). It is expected that after this review, which dates from 2014, many new apps were developed and validated. Apart from reviewing the new evidence from these validation studies, a meta-analysis on results across different validation studies, along with a critical 
evaluation of the study designs, could provide more information on the accuracy of using food record apps in real-life situations.

Thus, this systematic review aims to evaluate the current state of the overall accuracy of recent mobile phone dietary apps in estimating the intake of energy, macronutrients, micronutrients, and food groups, using a meta-analysis if applicable. Also, it aims to review the applied designs and methodological aspects of validation studies on mobile phone food record apps.

\section{Methods}

Studies published in English were identified from the online databases Web of Science, Medline, and PubMed, using the following search strategy from Jan. 1st, 2013 to Oct. 31st, 2019: [("smartphone" OR "phone" OR "telephone" OR "mobile" OR "app" OR "mobile app*”) AND ("diet* record" OR "dietary assessment" OR “ food intake" OR "dietary measurement" OR “energy intake" OR "caloric intake” OR "nutrient intake" OR "nutrition assessment" OR "diet tracking” OR "food tracking”) AND ("valid*” OR "accuracy" OR "compar*" OR "evaluat*") in abstract or title]. We also scrutinized citations from already detected studies and review articles.

\section{Study identification and data extraction}

Studies were potentially eligible for inclusion in this systematic review if they satisfied all of the following criteria: (1) exclusively self-reported mobile phone apps that simulate food records; (2) included a validation that compares the app to an objective method (e.g. biomarker or accelerometer) or with a reference dietary assessment method (e.g. 24HR, FFQ, etc.); (3) studies with a "real life" setting (a sample of participants entering all consumptions they consumed on a day in a free-living situation); (4) Validation studies covering any segments of the global population and all genders. Two researchers (AM, LZ) performed study screening independently and blinded in the web application Rayyan (15). After the first screening looking at titles and abstracts, agreement on the list of selected papers was reached between the reviewers. Full articles were then retrieved and were further assessed for eligibility, independently and blinded, by the two researchers. The final decision on the inclusion of studies was based on a consensus between the two researchers and discussed with MO (supervisor), if necessary. This systematic review protocol was developed following the Preferred Reporting Items for Systematic Reviews (PRISMA) statement (16).

The features and results of each validation study were extracted consecutively by two researchers (AM extracted the data, and LZ checked the data for accuracy and vice versa). General characteristics of the validation studies, such as the type of reference method, the choice of a timeframe, the sequence and spacing of test and reference methods, the selection 
and the number of subjects, and the applied statistical tests were extracted. Mean differences in energy and macronutrient intake were extracted between the test method (app) and the reference method for further meta-analysis. Energy intake was transformed into kcal if it was only available in kilojoules. For studies in which multiple days were compared, only the average of the total period or only data where the number of participants satisfied the power calculation for studies was taken into account (e.g., Chen et al.). The correlation coefficients (Pearson $r$ and Spearman rho) and limits of agreement (LOA) were collected where available. The correlation coefficients were categorized based on Chan (17) and Akoglu (18) into strong if $r \geq 0.80$, moderate if $0.60 \leq r<0.80$, fair if $0.30 \leq r<0.60$, poor if $r<0.30$. For studies where other nutrients and food groups were measured, correlation coefficients and under- or overreporting between the app and the reference methods are presented.

\section{Meta-Analysis}

The meta-analysis of energy and macronutrients was performed on studies that had enough uniformity of available data for the dietary component under analysis. Studies were included for meta-analysis if they presented a mean and standard deviation for the app and the reference method (so-called raw effect size data that was most consistent between reviewed studies), and their units for macronutrient were in grams. Pooled mean differences (and 95\% confidence intervals) between the app and the reference method were calculated using Hartung-Knapp-Sidik-Jonkman (HKSJ) random effect model. HKSJ has fewer false positives with a small number of studies than the more common DerSimonian-Laird estimator (19). $\mathrm{X}^{2}$ test (20) at the significance level of $\mathrm{p}<0.05$ was performed with the $\mathrm{I}^{2}$ statistic, in which cut-offs in between $25 \%$ to $50 \%, 50 \%$ to $75 \%$ and more than $75 \%$ indicate low, moderate, and high heterogeneity, respectively (21).

When the test showed significant heterogeneity, the sources of heterogeneity were explored with a stratification analysis by two characteristics of the validation study, i.e., the reference method used in the study and whether the same food composition table was used in the app and the reference method. Stratification was performed only on the validation of dietary components if the number of validation studies was ten or more.

Sensitivity analyses were conducted to examine the impact of outlier studies. The outliers were identified by: first, if the individual study's confidence interval did not overlap with the confidence interval of the pooled effect. Second, the Graphic Display of Heterogeneity (Gosh) Plot method was used to detect potential outliers, in case there were borderline studies that nearly non-overlapping with pooled confidence intervals (22). The test could detect studies which might potentially contribute to the heterogeneity. Sensitivity analysis was performed for the intake of both energy and macronutrients by omitting the outlier study. 
In the case of 10 or more contributing studies, the potential of publication bias was analyzed with Egger's test (23) for publication bias. Data were analyzed with the statistical program R-Studio ${ }^{\circledR}$ ver.1.2.5019, $\mathrm{R}{ }^{\circledR}$ ver. 3.6.1., R packages used include meta, metaphor, esc, and dmetar.

\section{Results}

The database searches yielded 825 publications when search results were combined, and two additional articles were identified through other sources (search alerts in searched databases). After duplicate records were removed, the title and abstract of 582 studies were screened, which resulted in the exclusion of 518 studies. After applying inclusion and exclusion criteria, 14 studies were selected for the systematic review, of which 11 studies were selected for meta-analysis on energy intake, and eight studies were selected for meta-analysis on macronutrient intake (see Figure 1).

Table 1 shows different app characteristics and design aspects regarding each validation study. The 14 studies focussed on 12 different apps, of which 7 provided feedback on nutrient intake (24-32) and 5 others did not (12,33-36). Most validation studies included young adults as their sample population or advertised in a university setting, while two studies explicitly mentioned to include a wider age range of participants $(26,35)$. Most validation studies had a medium to small sample size (from 18 to 81 participants), while two studies had a larger sample size of 362 and 189 participants $(26,33)$. The period of app use ranged mostly from 2 to 7 days and contained at least one weekend day for most studies, while two studies asked participants to record every day for three months $(24,26)$. The app use was on nonconsecutive days for three of the studies $(12,27,34)$. Ten studies used $24 \mathrm{HR}$ as the only reference method for two days $(n=6)(24,25,29,32,35,36)$ or three days $(n=4)(30,31,33$, $34)$. One study used a food frequency questionnaire (FFQ) (26), one study used food records (27), two studies used an accelerometer (to measure energy expenditure) $(12,28)$, and one study used a combination of accelerometer, 24HR, and food records (32). Among studies with different days of the app and the reference method, most studies compared the mean of each method averaged across all corresponding days (24, 30-32, 36). Apart from two studies using accelerometers exclusively $(12,28)$, five studies used different food composition databases (FCDs) for the app and the reference method (24-27, 33), and seven used the same FCD. Ten studies investigated the energy and macronutrient intake, while six of them also compared micronutrient intake $(24,26,30,33,34,36)$. Four studies looked at food group intakes $(31,32,34,35)$. In terms of statistical parameters and tests, the frequency of using pair t-test was the highest $(n=12)$, followed by correlation coefficient $(n=11)$ and BlandAltman limits of agreement $(n=11)$, Thirteen studies used at least two statistical parameters, eight studies used all three parameters in their studies, while Lee only used the t-test (24). 

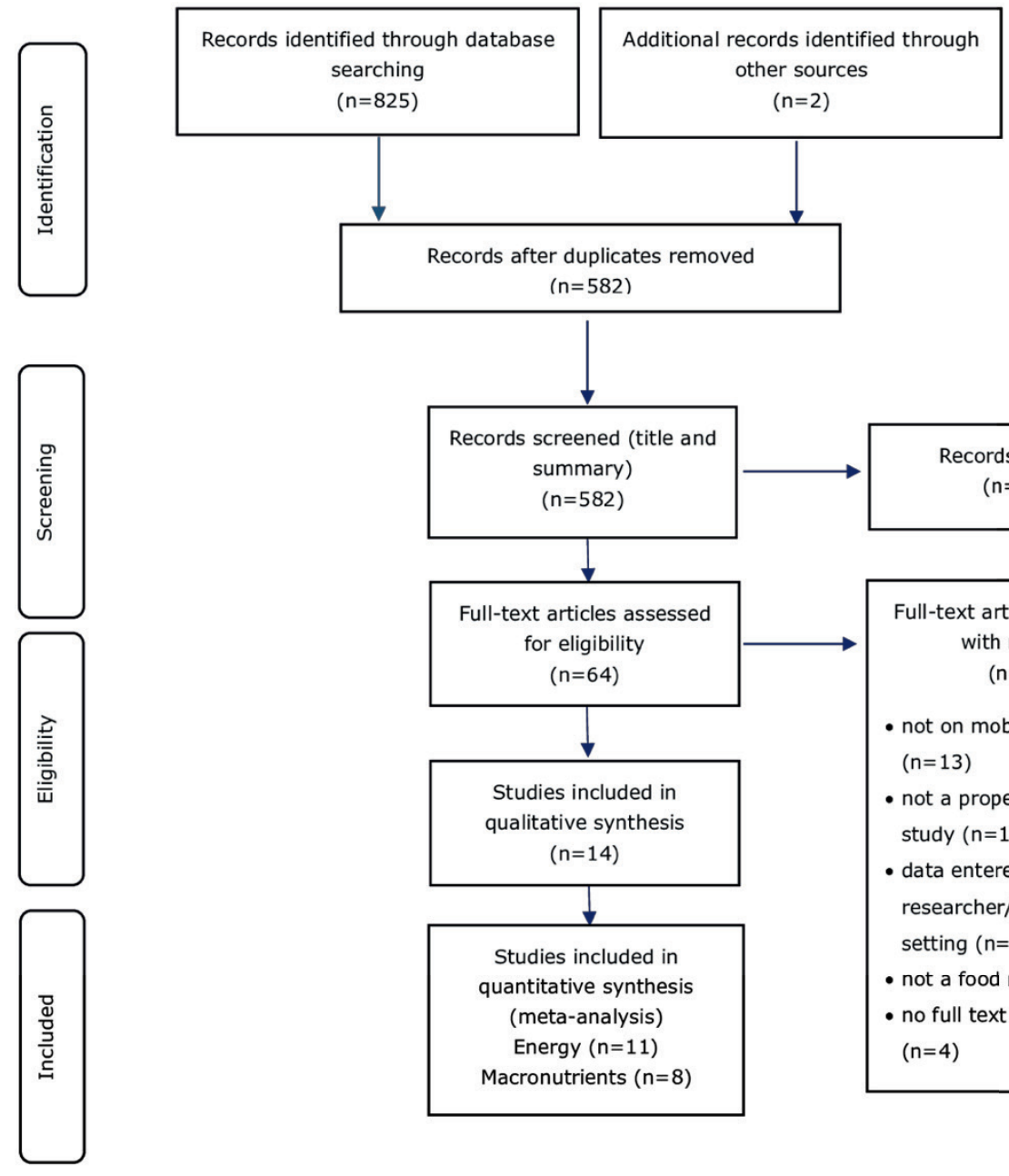

Records after duplicates removed

$$
(n=582)
$$
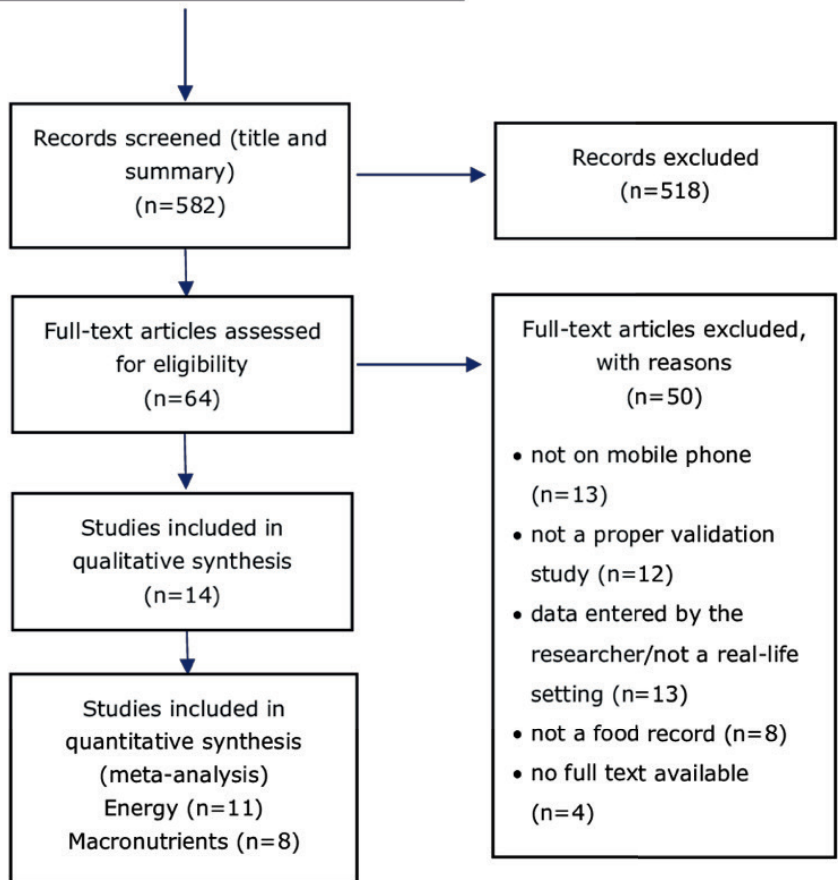

Figure 1. PRISMA Flow Diagram indicating the number of articles included at each phase 


\begin{tabular}{|c|c|c|c|c|c|c|c|}
\hline УOT & & $>$ & $>$ & $>$ & $>$ & $>$ & $>$ \\
\hline иоџ̣в|ә.лоつ & & $>$ & $>$ & $>$ & $>$ & $>$ & $>$ \\
\hline 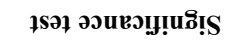 & $>$ & $>$ & $>$ & $>$ & $>$ & & $>$ \\
\hline \multicolumn{8}{|l|}{ sdno.do poo } \\
\hline słuว!.ฺ̣nuo.s!!W & m & & ฮิ & - & & & \\
\hline 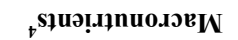 & in & t & $a$ & 6 & in & & \\
\hline Ко̊.юวи & $>$ & $>$ & $>$ & $>$ & $>$ & $>$ & $>$ \\
\hline $\begin{array}{c}\text { ¿spoчрәш } \\
\text { омч Ч! बつН әшеS }\end{array}$ & z & z & $\stackrel{\circ}{z}$ & $\stackrel{0}{z}$ & z & ' & ' \\
\hline 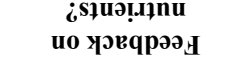 & $e^{\infty}$ & $\sum_{\lambda}^{\infty}$ & $\sum^{\infty}$ & $\stackrel{\circ}{z}$ & $\sum^{\infty}$ & $\stackrel{\circ}{z}$ & $\sum^{\infty}$ \\
\hline $\begin{array}{c}\text { Sरвp } \\
\text { рочџәш әәиә.әرәу }\end{array}$ & 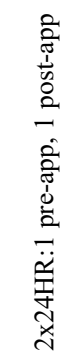 & 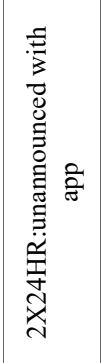 & 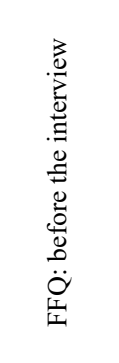 & 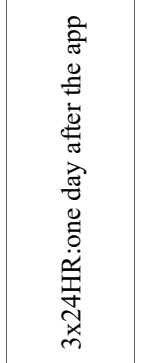 & 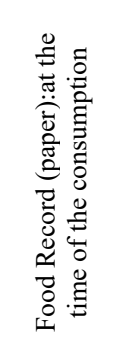 & 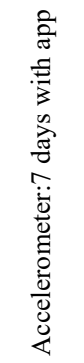 & 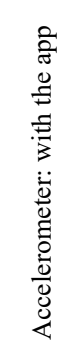 \\
\hline siepp ddV & 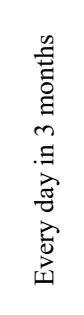 & 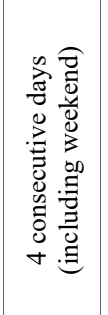 & 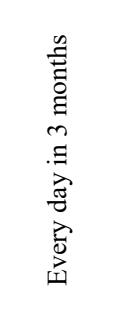 & 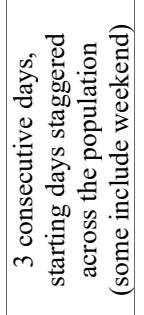 & 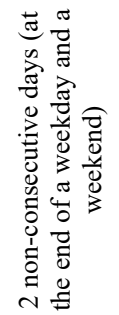 & 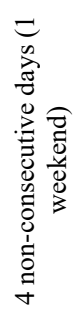 & 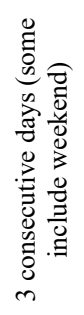 \\
\hline ә|durs Kpm & 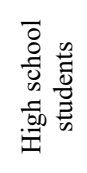 & 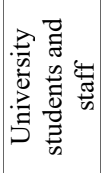 & 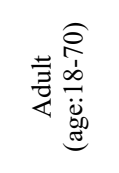 & 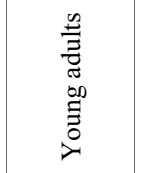 & 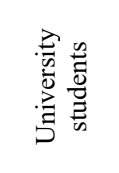 & 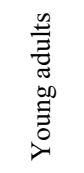 & 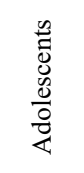 \\
\hline $\begin{array}{c}\text { u } \\
\text { K.นunoว }\end{array}$ & $\begin{array}{l}\mathscr{D} \\
\stackrel{0}{\mathbb{V}}\end{array}$ & 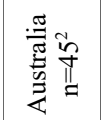 & 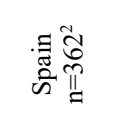 & 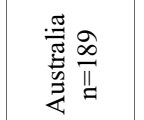 & 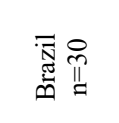 & 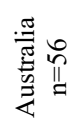 & 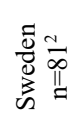 \\
\hline 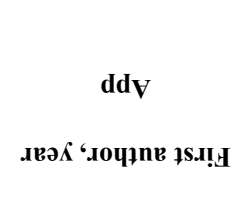 & 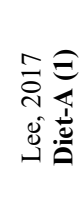 & 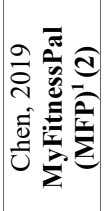 & 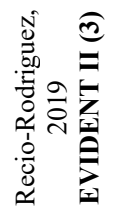 & 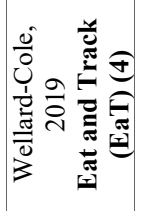 & 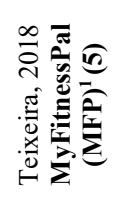 & 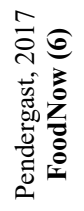 & 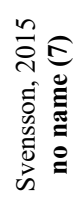 \\
\hline
\end{tabular}




\begin{tabular}{|c|c|c|c|c|c|c|c|c|}
\hline VOT & & $>$ & $>$ & $>$ & $>$ & $>$ & & \\
\hline иоџре|ว.лоว & $>$ & & $>$ & $>$ & $>$ & $>$ & & \\
\hline ұรә әวนвวџ!นภ̊!S & $>$ & $>$ & & $>$ & $>$ & $>$ & $>$ & \\
\hline sdno.do poo $_{4}$ & $\simeq$ & $\sim$ & & & & $\infty$ & - & \\
\hline słuə!.ฺnuo.s!ณ & $m$ & & N & & \pm & & & \\
\hline 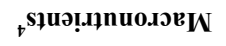 & 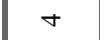 & $\theta$ & $\infty$ & + & 우 & & - & \\
\hline Ко̊.әй & $>$ & $>$ & $>$ & $>$ & $>$ & & & \\
\hline $\begin{array}{c}\text { ¿sрочрәш } \\
\text { омұ U! बつ马 әшеS }\end{array}$ & $\stackrel{\infty}{\infty}$ & $\stackrel{\infty}{\infty}$ & $\stackrel{\infty}{ٍ}$ & $\stackrel{\infty}{\circlearrowright}$ & $\sum^{\infty}$ & $\stackrel{\infty}{\nu}$ & $\overbrace{\nu}^{\infty}$ & \\
\hline 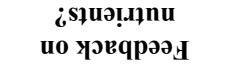 & z & z & z & $\stackrel{\infty}{\infty}$ & ${ }_{\lambda}^{3}$ & $\stackrel{y}{\infty}$ & $\stackrel{\infty}{\nu}$ & \\
\hline $\begin{array}{c}\text { sरиp } \\
\text { рочұәш әэиәләәу }\end{array}$ & 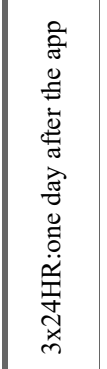 & 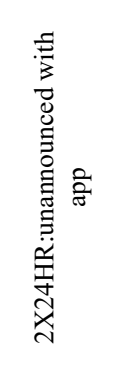 & 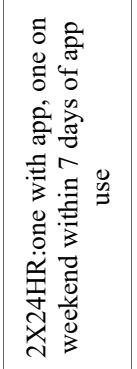 & 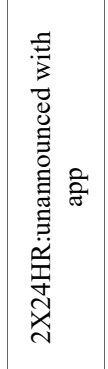 & 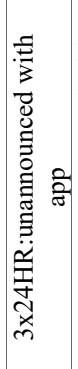 & 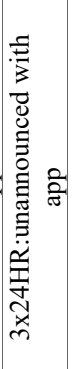 & 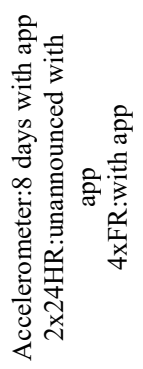 & \\
\hline siep ddV & 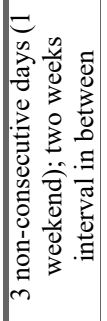 & 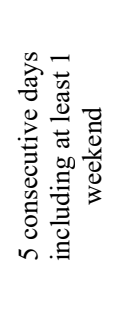 & 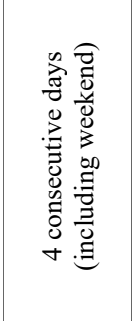 & 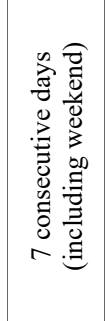 & 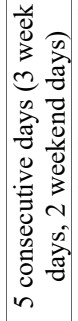 & 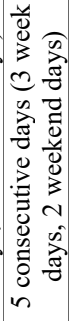 & 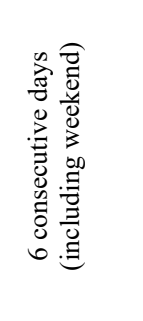 & 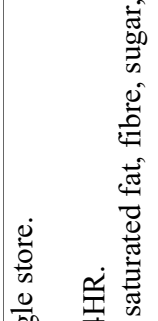 \\
\hline ә|durs $\mathrm{Kpm}$ S & 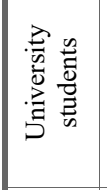 & 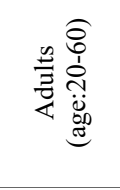 & 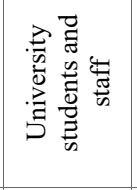 & 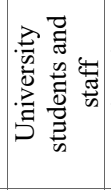 & 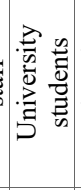 & 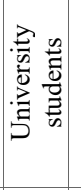 & 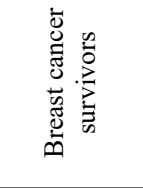 & 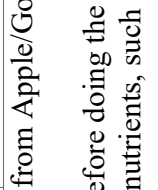 \\
\hline $\begin{array}{c}\text { U } \\
\text { K.ıฺUnoว }\end{array}$ & 需京 & 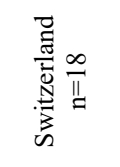 & 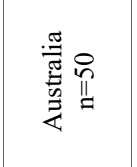 & 光売 & 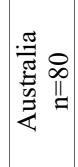 & 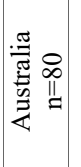 & 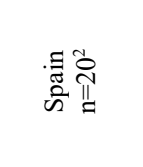 & 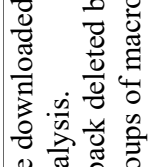 \\
\hline 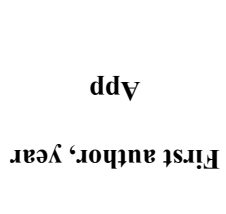 & 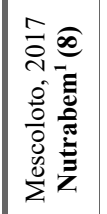 & 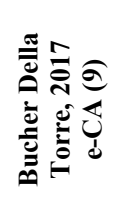 & 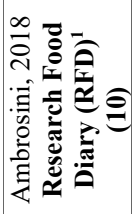 & 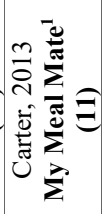 & 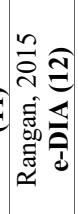 & 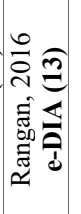 & 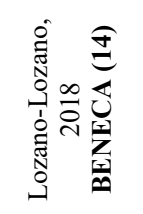 & 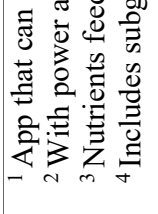 \\
\hline
\end{tabular}


Meta-analysis was performed on 11 studies for energy intake and eight studies for macronutrient intake. Figure 2A shows the pooling of the mean difference in energy. All apps underreported mean energy intake when compared to the reference method with a pooled effect of $-202 \mathrm{kcal}(95 \% \mathrm{CI}:-319$ to $-85 \mathrm{kcal})$. Heterogeneity expressed as $\mathrm{I}^{2}$ was $72 \%$, which fell into the upper-moderate to high heterogeneity group. Stratification was first performed between the eight studies that used $24 \mathrm{HR}$ as a reference method and the three studies that used all "other" reference methods. In the $24 \mathrm{HR}$ group, a lowered pooled mean difference of $-186 \mathrm{kcal}$ ( $95 \% \mathrm{CI}$ : -334 to $-37 \mathrm{kcal})$ was found, with a lowered heterogeneity $\left(\mathrm{I}^{2}=59 \%\right)$. Then stratification was performed on 12 studies that either used "the same" or "different" FCDs for the app and the reference method. The pooled mean difference in the group of studies with the same FCD decreased to $-57 \mathrm{kcal}(95 \% \mathrm{CI}$ : -116 to $2 \mathrm{kcal})$, the heterogeneity dropped to $0 \%$. Heterogeneity was also explored with sensitivity analysis to exclude outlying studies. No outliers were detected by looking at the overlapping of confidence intervals (CIs) of each study with the pooled effect. Using the Gosh Plots method the EVIDENT II app (26) was detected as an outlier. The pooled effect dropped to $-171 \mathrm{kcal}$ ( $95 \% \mathrm{CI}:-288$ to $-54 \mathrm{kcal}$ ), and the heterogeneity dropped to $\mathrm{I}^{2}=52 \%$ after deleting the outlier. Egger's test $(\mathrm{p}=0.17)$ indicated no evidence of study bias.

The pooling of the effect sizes on carbohydrate, fat, and protein intake was performed on eight studies (see Figure 2B, 2C, 2D). The pooled effects were negative for all three macronutrients. High heterogeneity of carbohydrate $\left(\mathrm{I}^{2}=86 \%\right)$ and protein $\left(\mathrm{I}^{2}=80 \%\right)$ was found, with the pooled effect of $-26.9 \mathrm{~g} /$ day and $-12.2 \mathrm{~g} /$ day, respectively. Similar to energy, the EVIDENT II app was detected as an outlier for carbohydrate (26). After deleting the data of the outlier, the heterogeneity dropped to moderate for carbohydrate $\left(\mathrm{I}^{2}=54 \%\right)$, with the pooled effect of $-18.8 \mathrm{~g} /$ day. The heterogeneity of fat was slightly lower than carbohydrate and protein $\left(\mathrm{I}^{2}=73 \%\right)$, with a pooled effect of $-12.7 \mathrm{~g} /$ day. In all eight studies, the app underreported mean fat intake when compared to the reference method.

When looked at the performance of each app, e-DIA had a relatively lower mean difference and variance in the intake of energy and all macronutrients than other apps (30). The app eCA had the lowest mean difference for both carbohydrate and protein (35). However, the standard deviation of the differences was the highest among all studies for energy, carbohydrate, and fat. Diet-A and MFP (Chen) had the highest mean difference across the energy, fat and protein $(24,25)$. Together with EVIDENT II app, which is the outlier for energy and carbohydrate, these three studies used different FCD for the app and the reference methods. 
A.

\begin{tabular}{|c|c|c|c|c|c|}
\hline \multirow[b]{2}{*}{ Study } & \multicolumn{2}{|c|}{ App (kcal/day) Reference (kcal/day) } & \multirow[b]{2}{*}{ Mean Difference (kcal/day) } & \multirow[b]{2}{*}{$95 \%-\mathrm{Cl}$} & \multirow[b]{2}{*}{ Weight } \\
\hline & Total Mean SD & Total Mean SD & & & \\
\hline Diet-A (Lee, 2017) & 211427.0379 .0 & 211893.0394 .0 & 曰 & $-466.0[-699.8 ;-232.2]$ & $8.5 \%$ \\
\hline MFP (Chen, 2019) & 451513.0530 .0 & 451958.0627 .0 & ㅍ & $-445.0[-684.9 ;-205.1]$ & $8.3 \%$ \\
\hline EVIDENT \| (Recio-Rodriguez, 2019) & 3622058.5557 .9 & 3622467.3729 .8 & - & $-408.8[-503.4 ;-314.2]$ & $12.8 \%$ \\
\hline EaT (Wellard-Cole, 2019) & $1892168.0 \quad 501.4$ & 1892377.9696 .9 & & $-209.8[-332.3 ;-87.4]$ & $120 \%$ \\
\hline FoodNow (Pendergast, 2017) & 562199.8467 .9 & $\begin{array}{lll}56 & 2397.3 & 528.3\end{array}$ & & $-197.5[-382.3 ;-12.7]$ & $10.0 \%$ \\
\hline Nutrabem (Mescoloto, 2017) & $40 \quad 1804.4 \quad 696.4$ & $40 \quad 1949.5560 .9$ & & $-145.1[-422.2 ; 132.0]$ & $7.3 \%$ \\
\hline e-CA (Bucher Della Torre, 2017) & 182287.0792 .0 & 182388.0664 .0 & & $-101.0[-578.5 ; 376.5]$ & $3.7 \%$ \\
\hline RFD (Ambrosini, 2018) & 502116.2541 .8 & 502180.4558 .6 & & $-64.3[-280.0,151.4]$ & $9.0 \%$ \\
\hline MMM (Carter, 2013) & 411917.0405 .0 & $41 \quad 1970.0403 .0$ & & $-53.0[-227.9 ; 121.9]$ & $10.3 \%$ \\
\hline MFP (Teixeira, 2018) & 301820.8509 .7 & $\begin{array}{llll}30 & 1834.2 & 481.4\end{array}$ & & $-13.4[-264.3,237.5]$ & $80 \%$ \\
\hline e-DIA (Rangan, 2015) & 801947.5596 .4 & 801955.6615 .5 & & $-8.1[-195.9 ; 179.7]$ & $9.9 \%$ \\
\hline Random effects model & 932 & 932 & & $-201.6[-318.5 ;-84.7$ & $100.0 \%$ \\
\hline \multirow{2}{*}{\multicolumn{3}{|c|}{ Heterogeneity. $I^{2}=72 \%[49 \%, 85 \%], p<0.01$}} & 11 & & \\
\hline & & & 200400600 & & \\
\hline
\end{tabular}

B.

\begin{tabular}{|c|c|c|c|c|c|c|c|c|}
\hline Study & Num & $\begin{array}{l}\mathrm{pp}(g / \text { day } \\
\text { Mean }\end{array}$ & ay) ${ }^{R}$ & $\begin{array}{l}\text { Refere } \\
\text { Num }\end{array}$ & $\begin{array}{l}\text { ence (g/day) } \\
\text { Mean SD }\end{array}$ & Mean Difference (g/day) & $95 \%-\mathrm{Cl}$ & Weight \\
\hline EVDENT I (Recio-Rodriguez, 2019) & 362 & 196.6 & 57.6 & 362 & 260.488 .1 & 母 & $-63.8[-74.6 ;-53.0]$ & $17.2 \%$ \\
\hline Diet-A (Lee, 2017) & 21 & 198.8 & 48.8 & 21 & 255.654 .6 & & $-56.8[-88.1 ;-25.5]$ & $12.3 \%$ \\
\hline MFP (Chen, 2019) & 45 & 158.0 & 90.0 & 45 & 198.076 .0 & 田 & $-40.0[-74.4 ;-5.6]$ & $11.5 \%$ \\
\hline MFP (Teixeira, 2018) & 30 & 207.8 & 72.1 & 30 & 232.963 .2 & & $-25.2[-59.5 ; 9.2]$ & $11.5 \%$ \\
\hline Nutrabem (Mescoloto, 2017) & 40 & 217.5 & 72.3 & 40 & 230.065 .5 & 巴 & $-12.5[-42.7 ; 17.8]$ & $12.6 \%$ \\
\hline MMM (Carter, 2013) & 41 & 245.0 & 47.0 & 41 & 248.056 .0 & 车 & $-3.0[-25.4 ; 19.4]$ & $14.6 \%$ \\
\hline e-CA (Bucher Della Torre, 2017) & 18 & 244.01 & 113.0 & 18 & 244.084 .0 & & $0.0[-65.0 ; 65.0]$ & $5.9 \%$ \\
\hline e-DIA (Rangan, 2015) & 80 & 213.3 & 82.6 & 80 & 209.067 .5 & & $4.3[-19.1 ; 27.7]$ & $14.4 \%$ \\
\hline \multirow{2}{*}{\multicolumn{2}{|c|}{$\begin{array}{l}\text { Random effects model } \\
\text { Heterogeneity } I^{2}=86 \%[74 \% ; 92 \%], p<0\end{array}$}} & & & 637 & & & $-26.9[-49.8 ;-4.0]$ & $100.0 \%$ \\
\hline & & & & & & .50 & & \\
\hline
\end{tabular}

C.

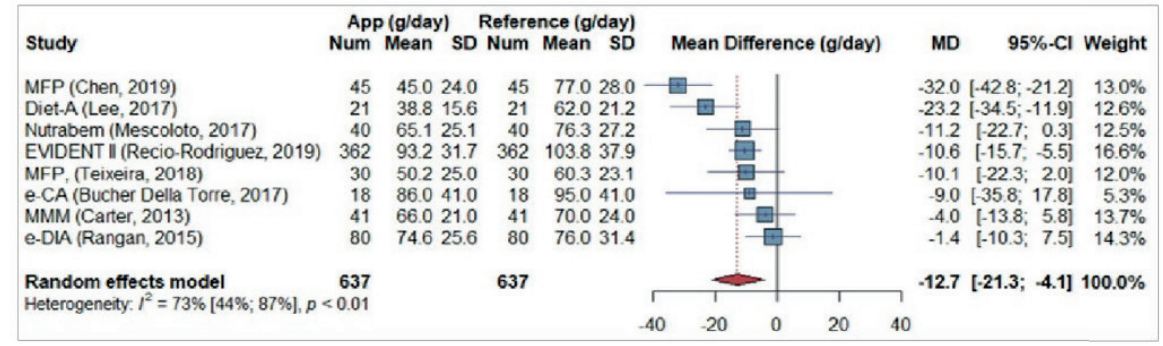

D.

\begin{tabular}{|c|c|c|c|c|c|c|c|c|}
\hline Study & $\begin{array}{l}\text { App } \\
\text { Num }\end{array}$ & $\begin{array}{l}\text { (g/day) } \\
\text { Mean SD }\end{array}$ & $\begin{array}{l}\text { Refere } \\
\text { Num }\end{array}$ & $\begin{array}{l}\text { nce (g/day) } \\
\text { Mean SD }\end{array}$ & Mean Difference ( $g /$ day) & MD & $95 \%-\mathrm{Cl}$ & Weight \\
\hline MFP & 45 & 0 & 45 & 89.03 & & -280 & {$[-425 ;-135]$} & $11.7 \%$ \\
\hline Diet- & 21 & 4 & 21 & 2 & & & $-39.3 ;-12.1]$ & \\
\hline EVIDENT I (Recio-Rodriguez, 2019) & 362 & 85.4219 & 362 & 106.929 .5 & 巴 & -21.5 & $-25.3 ;-17.7$ & $17.1 \%$ \\
\hline MFP $(T$ & 30 & 77.728 .7 & 30 & 88.112 & & -10.5 & $1-21.7 ; 0.7$ & $13.6 \%$ \\
\hline MMI & 41 & 17.0 & 41 & 76.0 & & -4.0 & {$[-11.6 ; 3.6]$} & $15.5 \%$ \\
\hline $\mathrm{e}-\mathrm{Dl}$ & 80 & 887335 & 80 & 91.3 & & .26 & {$[-132 ; 80$} & $139 \%$ \\
\hline Torre, 2017) & 18 & 88.0280 & 18 & 86.029 .0 & & 2.0 & {$[-16.6 ; 20.6]$} & $9.6 \%$ \\
\hline Nutrabem (Mescoloto, 2017 & 40 & 88.777 .2 & 40 & 86.639 .2 & & 2.1 & {$[-24.8 ; 28.9]$} & $6.4 \%$ \\
\hline $\mathbf{R}$ & 637 & & 637 & & & -12.2 & [-22.1; & $100.0 \%$ \\
\hline \multicolumn{2}{|l|}{ Heler } & & & & 20 & & & \\
\hline
\end{tabular}

Figure 2. Forest plot for the mean difference in energy and macronutrient intake between the app and the reference method in included validation studies. A. Energy, B. Carbohydrate, C. Fat, D. Protein. 
Table 2 illustrates the correlation coefficient and limits of agreement (LOA) between the apps and the reference methods for the intake of energy and macronutrients. The column with LOA represents the distance between the upper and the lower limit. Five studies reported both correlation and LOA for energy and all macronutrients. For energy, the three studies that had a weak correlation between two methods, had larger LOAs than other studies $(25,26,28)$. Most studies had a moderate correlation with a range of 0.60 to 0.80 . The distances of LOA were mostly within $2000 \mathrm{kcal}$, with one exception of $2223 \mathrm{kcals}$. Nutrabem had the highest correlation for energy, carbohydrate, and protein (34). MMM had the highest correlation in fat (29). The app e-Dia had similar correlations for energy and all macronutrients from 0.64 to 0.79 (30). EVIDENT II had weak correlations for all macronutrients and energy (26). The average correlation across studies was 0.54 to 0.60 , energy and fat intake were both the lowest at 0.54 . The average across energy and macronutrients in each study ranged from 0.23 to 0.78 , with majority studies in the moderate category. The expression of macronutrient intake differed between studies, with grams, energy percentages, and natural logarithms.

Tables 3 lists other nutrients that were most commonly assessed in the included studies. In most studies, the app underestimated nutrient intakes. Calcium and sodium intake in Diet-A, fiber, and alcohol in EVIDENT II were significantly underestimated while the rest of the underestimated nutrients were all non-significant. Alcohol intake was significantly overestimated in RFD. Rangan compared all nutrients in this table and had the secondhighest average correlation among the nutrients, while EVIDENT II had the lowest average correlation across most nutrients, except alcohol. EaT had the highest average correlation among the included nutrients, mainly due to the strong correlation for sugar intake.

Food groups were only validated for four apps (e-CA, Nutrabem, BENECA, e-Dia). A different categorization of food groups was found across studies, differences in dairy, fruits, vegetables, meat, and grain intake, were most commonly reported. Food group intakes were mostly insignificantly underestimated by apps. In the BENECA-app vegetables and fruits were mostly forgotten by participants. Among studies investigated correlations, the highest correlation found for Nutrabem-app was poultry $(\mathrm{r}=0.85)$ and lowest in nuts $(\mathrm{r}=0.31)$ and vegetable oils $(\mathrm{r}=0.37)$. The app e-DIA had relatively stronger correlations among all included food groups, from 0.75 to 0.88 , and has an equal number of under- and overestimations. 


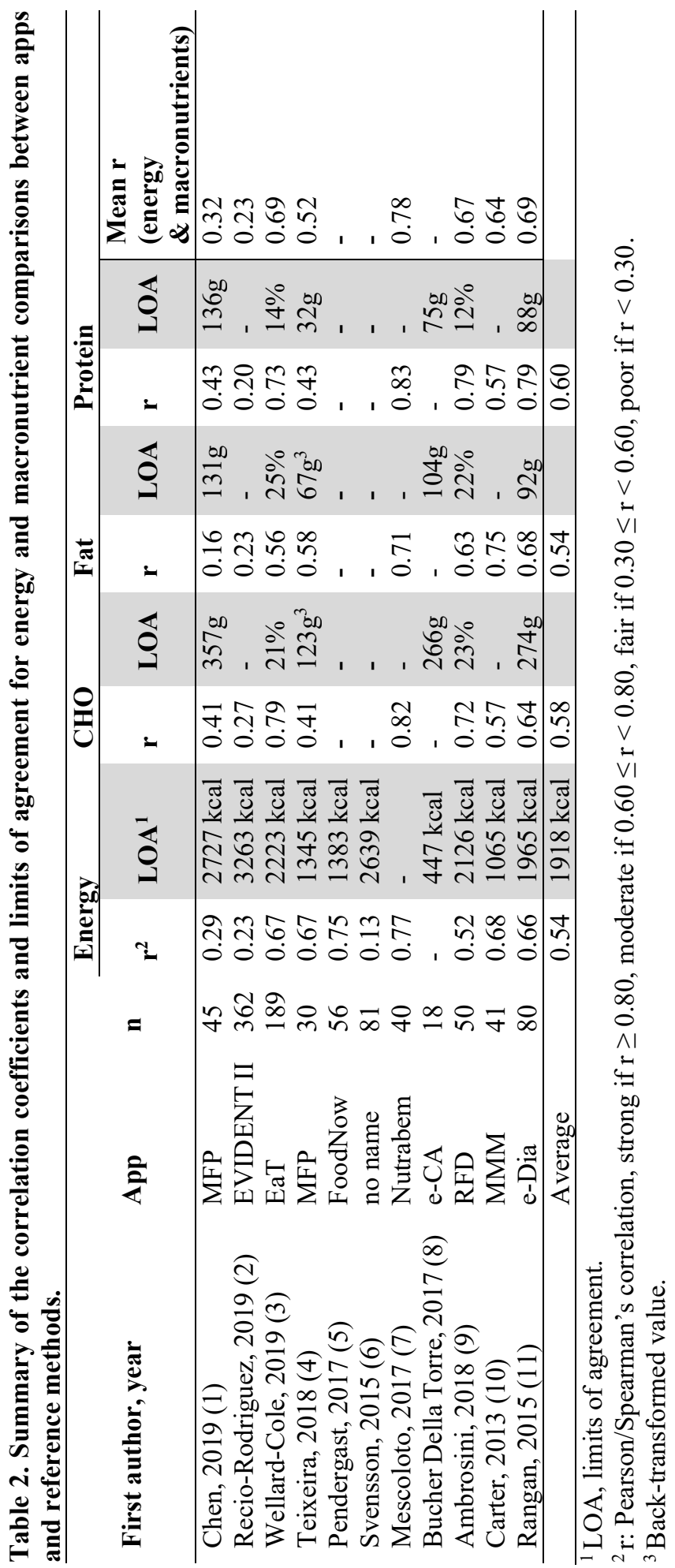




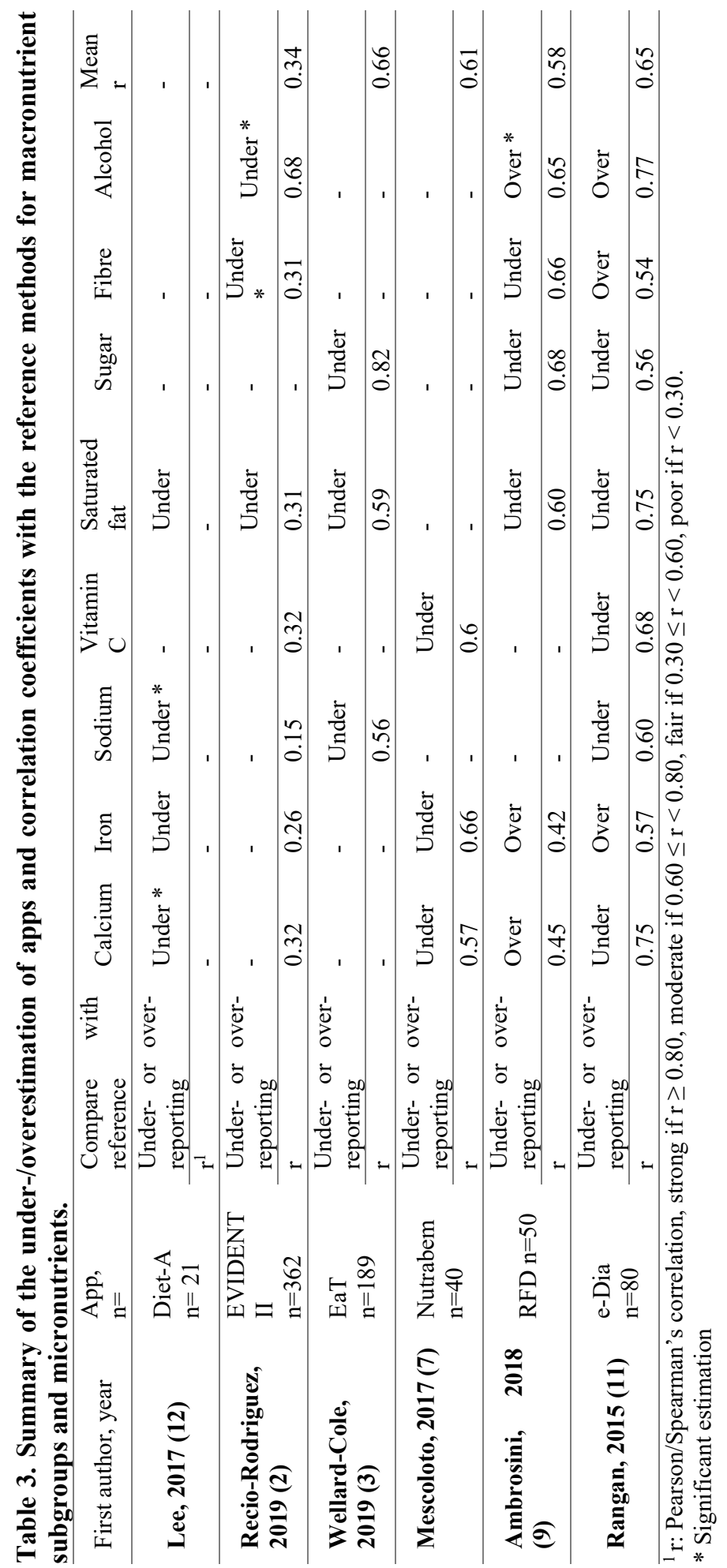




\section{Discussion}

This paper aimed to assess the overall accuracy of dietary intake measurements in validated mobile phone food record apps. Apps from more than half of the 14 included studies were validated in university settings, were small scale with a duration of 2 to 7 consecutive days, used 24HR as the reference method, and used the same FCDs for the test and the reference method. The meta-analysis on results for 8-10 apps found that food record apps underreported energy and macronutrients relative to classical dietary assessment methods. Moderate heterogeneity was reached when an outlier study was excluded from the metaanalysis for energy and carbohydrate. Studies using the same food composition database for the apps and the reference methods had no heterogeneity for energy intake and had a lowered pooled effect of $-57 \mathrm{kcal}$. Studies that observed smaller differences in energy intake between the app and the reference method also had smaller differences in macro- and/or micronutrients and food groups.

\section{Intentional/Unintentional Underreporting}

Underreporting of energy intake in the app compared to the reference method was found in all studies. An even larger extent of under-reporting was expected for studies that used an objective reference method as the reference, because underreporting is also often observed in the 24HR (8), which most studies have used. The tendency of underreporting when using the app or other self-reporting methods may either be unintentional and intentional (11). The effect of unintentional underreporting could potentially be alleviated by adding adequate prompts and improving technological add-ins (36). Intentional underreporting is more challenging to eliminate when participants deliberately omit the input of certain foods out of social acceptability or convenience or temporarily change their eating behaviour (37). In the current study, a larger extent of underestimation in carbohydrate and fat intake was found as compared to protein, which is in line with the findings from another review on a technologybased dietary assessment tool by Eldridge et al. (8). Bucher Della Torre et al. and Chen et al. found that people tend to underreport fat, alcohol, discretionary foods and beverages (high in fat/sugar) intake unless prompted by interviewers $(25,35)$, while Rangan et al. indicated the underreporting of added sugar and alcohol might be due to intentional underreporting of foods containing added sugars or the reduced alcohol or sugar intake while using the app (30).

Approximately half of the errors in energy intake estimations from dietary records administered on technological devices have been attributed to wrong portion size estimations (38). Participants were asked to refer to a provided food model booklet to assist with the estimation of portion sizes during 24HRs, while most apps provide metric weights (e.g., g, $\mathrm{mL}$ ) or household measure options (e.g., cups) with no images accompanied (39). Bucher 
Della Torre et al. found that participants tended to choose the app proposed portions even if their real portions are different, especially with drinks (35). Mobile technologies with the assistance of digital photographs have shown less extent of underestimation than regular food records in a free-living situation compared to doubly labelled water (DLW) (40-42). These studies were not included in the current review because they were not exclusively selfreporting, and required a large involvement of dietitians to identify foods and amounts from photos correctly. Automatic food recognition and volume estimation could potentially outperform portion sizes estimated by individuals, but validations are needed to verify their applicability in large-scale studies (43).

Some studies conducted the 24HR the next day of using the app, which might have caused a memory effect and lessened the recalling bias of 24HR (29). Besides, access to the nutrient feedbacks from some apps could enhance health consciousness and induce changes in the food intake of those who are motivated for weight reduction $(44,45)$. Both study designs could lead to an increased agreement and augmented correlation between the two methods (35). The learning effect could be reduced if the app and the reference methods are used on separate days, with the app used first (46). Differences due to day-to-day variation in the data could be evened out with repeated measurements or corrected with statistical modelling (47). However, the source of variation (e.g., food omission, portion underestimation) could be investigated better if both methods were conducted on the same day. Moreover, unannounced $24 \mathrm{HR}$ is preferred to avoid behavioural change (48). Ambrosini et al. conducted the second 24HR unannounced on a different day within seven days of app use (36). In this way, both the app and reference method are measuring dietary intake to a similar extent while limiting the possible influence of each method.

\section{Explanations on High Heterogeneity}

We observed a higher mean difference in studies where different FCDs were incorporated into the app and the reference method. In studies where the same food items are entered by researchers into different apps, disagreements between apps is mainly due to the different FCDs embedded in each app $(8,49-53)$. Thus, the "human components", that were mainly accounted for in validation studies of methods rather than nutrient content, should be distinguished from different FCD use. If using the same FCD is impractical, comparing differences in food groups or food items between two methods could be a solution. Moreover, insight in validity of food groups can give some clues on specific foods that are easily forgotten, like the fat used for frying. Besides, advocacy to move from nutrient focus towards food-based research in nutrition epidemiology has stressed the importance of food group validation using new methods (54). Unfortunately, only four of the included studies validated food groups, and none of the studies that used different FCDs have considered comparing food groups. Moreover, studies with food group comparisons used different food 
categorizations and statistical tests, which limited the comparisons of food group differences across studies.

Our results indicated that the choice of the reference method was also one of the determining factors for heterogeneity. The absolute validity was not reported in smartphone application validations, possibly due to the high cost associated with recovery biomarkers and the availability for limited nutrients. When investigating the relative validity of a method it is desirable to use a reference method with uncorrelated errors and better accuracy, for example, comparing food records with 24HR. One study in the meta-analysis used FFQ as the reference, which has a lower level of accuracy with a limited frequency of consumption options and food lists in the FFQ tool (55). Furthermore, FFQs estimate nutritional intake over a longer time period (usual consumption) while more diverse food item options are influenced by seasonality of different foods. Conversely, Teixeira et al. tested their app with a paper-based food record measuring the food consumption of the same days. Here an overestimation of correlation was expected because two methods share the same embedded errors (27). Two studies used an accelerometer to assess energy expenditure, which is an objective measure less burdensome than DLW (12). However, accelerators have shown overand underestimation of energy expenditure when different types of physical activities were performed (56).

Most studies used a diverse range of statistical techniques that could facilitate a balanced interpretation of results (30). Correlation coefficients indicate the ability of the app to rank individuals and the strength of the association. Bland-Altman plots reveal the presence, direction, and extent of bias at the group level and the extent of measurement error at the individual level (57). A wide LOA found in most studies was expected because the reference measure itself might have potential errors and is not reflecting true intakes (29). Besides, only a few days of intake were collected for most studies. Rangan et al. found a smaller difference and a higher correlation with values adjusted for within-person variation. Garden et al. also found that the heterogeneity of FFQ validation studies decreased if deattenuated/energy-adjusted values were used (58). Because the majority of studies in this review did not adjust for the nutrient intake, only studies with raw data were compared. Hence, presenting data in several ways is necessary for cross-study comparisons and in obtaining insight into different types of error, i.e., systematic and random error (59).

Limited information was provided by included studies on whether they aimed at validating current or usual dietary intake. Although a single day food intake can be useful for many studies, usual intake is of primary interest for studies on surveillance, epidemiology and intervention (60). To measure the ability of an app to capture usual intake, studies that used $24 \mathrm{HR}$ and FFQ as the reference should be conducted on non-consecutive days, including both weekdays and weekend days $(51,55)$, which might capture more variations in diet and 
occasionally consumed foods, such as alcohol (12). A higher reporting accuracy of food records has been found when a weekend day was included. It was speculated that participants have more time during the weekend to complete a food record (61). In half of the included studies, participants used the app for less than four days, which was not sufficient to estimate usual micronutrients intake accurately and to capture habitual diet $(62,63)$, especially with a sample size less than fifty (64). To be fair, the limited number of studies that investigated and compared micronutrient intake indicated that it is still too premature to get insight in validity of micronutrient intake of apps. The inclusion of mostly young adults from university settings limited the generalizability of the validation results. Furthermore, in the case where people with low technological literacy used the apps, they probably provide less reliable data $(48,63,65)$.

\section{Strength and Limitations}

This study is the first meta-analyses of the validations of food record apps in free-living conditions; it provides a detailed comparison of the study design, and it includes results on micronutrients and food groups. For this study, a systematic search strategy for three electronic databases was adopted in searching for eligible papers, and we have not found no evidence of publication bias among the included studies. Still, we could not rule out the possibility that other eligible papers that are not in English or not available via electronic databases were missed. The exclusion of image-based mobile technologies (entered by dietitians) helped us to better understand the suboptimal performances among individuals using apps in naturalistic setting compared to studies entered by dietitians. The narrowed study selection criteria promoted a higher quality of reporting validity of dietary apps and allowed an easier comparison between studies. Another strength of the study was that heterogeneity on energy intake was explained by the stratification analysis, unfortunately, due to the small number of studies, testing for publication bias and exploring heterogeneity with stratification was only possible for energy intake. Moreover, the limited number of studies might lowered the power of the meta-analysis (58).

\section{Conclusions}

Food record apps underreport energy intake, as well as intake of macronutrients. No specific conclusions could be made on micronutrient and food group comparisons due to limited and incomparable data. Future validation studies should consider applying biomarkers as the reference method next to repeated 24HRs; include larger and more representative study populations, and should try to provide insight in the source of the measurement error by also looking at the validity of food groups and micronutrients. 


\section{References}

1. Arens-Volland AG, Spassova L, Bohn T. Promising approaches of computersupported dietary assessment and management-Current research status and available applications. Int J Med Inform. 2015 Dec;84(12):997-1008.

2. Naska A, Lagiou A, Lagiou P. Dietary assessment methods in epidemiological research: current state of the art and future prospects. F1000Res. 2017;6:926.

3. EFSA. General principles for the collection of national food consumption data in the view of a pan-European dietary survey. EFSA Journal. 2009;7(12):1435.

4. Hedrick VE, Dietrich AM, Estabrooks PA, Savla J, Serrano E, Davy BM. Dietary biomarkers: advances, limitations and future directions. Nutr J. 2012;11:109.

5. Shim J-S, Oh K, Kim HC. Dietary Assessment Methods in Epidemiologic Studies. Epidemiology and Health. 2014;36:e2014009.

6. Coulston AM, Boushey CJ. Nutrition in the prevention and treatment of disease. Amsterdam: Academic Press; 2008.

7. Dietary Assessment: A resource guide to method selection and application in low resource settings. Rome: FAO, 2018.

8. Eldridge AL, Piernas C, Illner AK, Gibney MJ, Gurinovic MA, de Vries JHM, et al. Evaluation of New Technology-Based Tools for Dietary Intake Assessment-An ILSI Europe Dietary Intake and Exposure Task Force Evaluation. Nutrients. 2018 Dec $28 ; 11(1)$.

9. Sharp DB, Allman-Farinelli M. Feasibility and validity of mobile phones to assess dietary intake. Nutrition. 2014;30(11):1257-66.

10. Allman-Farinelli M. Using digital media to measure diet. CAB Reviews: Perspectives in Agriculture, Veterinary Science, Nutrition and Natural Resources. 2018;13.

11. Illner AK, Freisling H, Boeing H, Huybrechts I, Crispim SP, Slimani N. Review and evaluation of innovative technologies for measuring diet in nutritional epidemiology. Int J Epidemiol. 2012 Aug;41(4):1187-203.

12. Pendergast FJ, Ridgers ND, Worsley A, McNaughton SA. Evaluation of a smartphone food diary application using objectively measured energy expenditure. Int J Behav Nutr Phys Act. 2017 Mar 14;14(1):30.

13. Serra-Majem L, Frost Andersen L, Henrique-Sanchez P, Doreste-Alonso J, SanchezVillegas A, Ortiz-Andrelluchi A, et al. Evaluating the quality of dietary intake validation studies. Br J Nutr. 2009 Dec;102 Suppl 1:S3-9.

14. Willett W, Lenart E. Reproducibility and validity of food-frequency questionnaires. Nutritional epidemiology. 2013:96-141.

15. Ouzzani M, Hammady H, Fedorowicz Z, Elmagarmid A. Rayyan-a web and mobile app for systematic reviews. Systematic reviews. 2016 Dec 5;5(1):210.

16. Moher D, Liberati A, Tetzlaff J, Altman DG, Group P. Preferred reporting items for systematic reviews and meta-analyses: the PRISMA statement. J Clin Epidemiol. 2009 Oct;62(10):1006-12. 
17. Chan YH. Biostatistics 104: correlational analysis. Singapore medical journal. 2003 Dec;44(12):614-9.

18. Akoglu H. User's guide to correlation coefficients. Turkish journal of emergency medicine. 2018 Sep;18(3):91-3.

19. Rover C, Knapp G, Friede T. Hartung-Knapp-Sidik-Jonkman approach and its modification for random-effects meta-analysis with few studies. BMC medical research methodology. 2015 Nov 14;15:99.

20. Fleiss JL. The statistical basis of meta-analysis. Stat Methods Med Res. 1993;2(2):121-45.

21. Higgins JP, Thompson SG, Deeks JJ, Altman DG. Measuring inconsistency in metaanalyses. BMJ. 2003;327(7414):557-60.

22. Olkin I, Dahabreh IJ, Trikalinos TA. GOSH - a graphical display of study heterogeneity. Research synthesis methods. 2012 Sep;3(3):214-23.

23. Egger M, Davey Smith G, Schneider M, Minder C. Bias in meta-analysis detected by a simple, graphical test. Bmj. 1997 Sep 13;315(7109):629-34.

24. Lee JE, Song S, Ahn JS, Kim Y, Lee JE. Use of a Mobile Application for SelfMonitoring Dietary Intake: Feasibility Test and an Intervention Study. Nutrients. 2017 Jul 13;9(7).

25. Chen J, Berkman W, Bardouh M, Ng CYK, Allman-Farinelli M. The use of a food logging app in the naturalistic setting fails to provide accurate measurements of nutrients and poses usability challenges. Nutrition. 2019;57:208-16.

26. Recio-Rodriguez JI, Rodriguez-Martin C, Gonzalez-Sanchez J, Rodriguez-Sanchez E, Martin-Borras C, Martinez-Vizcaino V, et al. EVIDENT Smartphone App, a New Method for the Dietary Record: Comparison With a Food Frequency Questionnaire. JMIR Mhealth Uhealth. 2019 Feb 8;7(2):e11463.

27. Teixeira V, Voci SM, Mendes-Netto RS, da Silva DG. The relative validity of a food record using the smartphone application MyFitnessPal. Nutr Diet. 2018 Apr;75(2):219-25.

28. Svensson A, Larsson C. A Mobile Phone App for Dietary Intake Assessment in Adolescents: An Evaluation Study. JMIR Mhealth Uhealth. 2015 Nov 3;3(4):e93.

29. Carter MC, Burley VJ, Nykjaer C, Cade JE. 'My Meal Mate' (MMM): validation of the diet measures captured on a smartphone application to facilitate weight loss. Br J Nutr. 2013 Feb 14;109(3):539-46.

30. Rangan AM, O'Connor S, Giannelli V, Yap MLH, Ming L, Roy R, et al. Electronic Dietary Intake Assessment (e-DIA): Comparison of a Mobile Phone Digital Entry App for Dietary Data Collection With 24-Hour Dietary Recalls. JMIR MHEALTH AND UHEALTH. 2015 2015--2015;3(4):3-14.

31. Rangan AM, Tieleman L, Louie JC, Tang LM, Hebden L, Roy R, et al. Electronic Dietary Intake Assessment (e-DIA): relative validity of a mobile phone application to measure intake of food groups. Br J Nutr. 2016 Jun;115(12):2219-26. 
32. Lozano-Lozano M, Galiano-Castillo N, Martin-Martin L, Pace-Bedetti N, FernandezLao C, Arroyo-Morales M, et al. Monitoring Energy Balance in Breast Cancer Survivors Using a Mobile App: Reliability Study. JMIR mHealth and uHealth. 2018 2018--Mar;6(3):e67-e.

33. Wellard-Cole L, Chen J, Davies A, Wong A, Huynh S, Rangan A, et al. Relative Validity of the Eat and Track (EaT) Smartphone App for Collection of Dietary Intake Data in 18-to-30-Year Olds. Nutrients. 2019 Mar 14;11(3).

34. Mescoloto SB, Caivano S, Domene SMA. Evaluation of a mobile application for estimation of food intake. Revista De Nutricao-Brazilian Journal of Nutrition. 2017 Jan-Feb;30(1):91-8.

35. Bucher Della Torre S, Carrard I, Farina E, Danuser B, Kruseman M. Development and Evaluation of e-CA, an Electronic Mobile-Based Food Record. Nutrients. 2017 Jan 18;9(1).

36. Ambrosini GL, Hurworth M, Giglia R, Trapp G, Strauss P. Feasibility of a commercial smartphone application for dietary assessment in epidemiological research and comparison with 24-h dietary recalls. Nutr J. 2018 Jan 9;17(1):5.

37. Scagliusi FB, Polacow VO, Artioli GG, Benatti FB, Lancha AH, Jr. Selective underreporting of energy intake in women: magnitude, determinants, and effect of training. J Am Diet Assoc. 2003 Oct;103(10):1306-13.

38. Beasley J, Riley WT, Jean-Mary J. Accuracy of a PDA-based dietary assessment program. Nutrition. 2005 Jun;21(6):672-7.

39. Subar AF, Crafts J, Zimmerman TP, Wilson M, Mittl B, Islam NG, et al. Assessment of the accuracy of portion size reports using computer-based food photographs aids in the development of an automated self-administered 24-hour recall. J Am Diet Assoc. 2010 Jan;110(1):55-64.

40. Martin CK, Han HM, Coulon SM, Allen HR, Champagne CM, Anton SD. A novel method to remotely measure food intake of free-living individuals in real time: the remote food photography method. British Journal of Nutrition. 2009 Feb;101(3):44656.

41. Rollo ME, Ash S, Lyons-Wall P, Russell AW. Evaluation of a mobile phone imagebased dietary assessment method in adults with type 2 diabetes. Nutrients. 2015;7(6):4897-910.

42. Delisle Nystrom C, Forsum E, Henriksson H, Trolle-Lagerros Y, Larsson C, Maddison R, et al. A Mobile Phone Based Method to Assess Energy and Food Intake in Young Children: A Validation Study against the Doubly Labelled Water Method and 24 h Dietary Recalls. Nutrients. 2016 Jan 15;8(1).

43. Daugherty BL, Schap TE, Ettienne-Gittens R, Zhu FM, Bosch M, Delp EJ, et al. Novel technologies for assessing dietary intake: evaluating the usability of a mobile telephone food record among adults and adolescents. J Med Internet Res. 2012 Apr $13 ; 14(2): \mathrm{e} 58$. 
44. Afshin A, Babalola D, McLean M, Yu Z, Ma W, Chen CY, et al. Information Technology and Lifestyle: A Systematic Evaluation of Internet and Mobile Interventions for Improving Diet, Physical Activity, Obesity, Tobacco, and Alcohol Use. Journal of the American Heart Association. 2016 Aug 31;5(9).

45. Forster H, Walsh MC, Gibney MJ, Brennan L, Gibney ER. Personalised nutrition: the role of new dietary assessment methods. Proc Nutr Soc. 2016 Feb;75(1):96-105.

46. Nelson M. The validation of dietary assessment. Design concepts in nutritional epidemiology. 1997;2:241-56.

47. Freedman LS, Midthune D, Dodd KW, Carroll RJ, Kipnis V. A statistical model for measurement error that incorporates variation over time in the target measure, with application to nutritional epidemiology. Stat Med. 2015 Nov 30;34(27):3590-605.

48. Walton J. Dietary Assessment Methodology for Nutritional Assessment: A Practical Approach. Top Clin Nutr. 2015;30(1):33-46 10.1097/TIN.0000000000000018.

49. Ferrara G, Kim J, Lin S, Hua J, Seto E. A Focused Review of Smartphone DietTracking Apps: Usability, Functionality, Coherence With Behavior Change Theory, and Comparative Validity of Nutrient Intake and Energy Estimates. JMIR Mhealth Uhealth. 2019 May 17;7(5):e9232.

50. Conrad J, Nothlings U. Innovative approaches to estimate individual usual dietary intake in large-scale epidemiological studies. Proc Nutr Soc. 2017 Aug;76(3):213-9.

51. Franco RZ, Fallaize R, Lovegrove JA, Hwang F. Popular Nutrition-Related Mobile Apps: A Feature Assessment. JMIR Mhealth Uhealth. 2016 Aug 01;4(3):e85.

52. Chen J, Cade JE, Allman-Farinelli M. The Most Popular Smartphone Apps for Weight Loss: A Quality Assessment. JMIR Mhealth Uhealth. 2015 Dec 16;3(4):e104.

53. Zhang L, Nawijn E, Boshuizen H, Ocke M. Evaluation of the Recipe Function in Popular Dietary Smartphone Applications, with Emphasize on Features Relevant for Nutrition Assessment in Large-Scale Studies. Nutrients. 2019 Jan 19;11(1).

54. Jacobs DR, Jr., Tapsell LC. Food, not nutrients, is the fundamental unit in nutrition. Nutr Rev. 2007 Oct;65(10):439-50.

55. Hooson J, Hutchinson J, Warthon-Medina M, Hancock N, Greathead K, Knowles B, et al. A systematic review of reviews identifying UK validated dietary assessment tools for inclusion on an interactive guided website for researchers: www.nutritools.org. Crit Rev Food Sci Nutr. 2019:1-25.

56. Bhammar DM, Sawyer BJ, Tucker WJ, Lee JM, Gaesser GA. Validity of SenseWear (R) Armband v5.2 and v2.2 for estimating energy expenditure. J Sports Sci. 2016 Oct;34(19):1830-8.

57. Lombard MJ, Steyn NP, Charlton KE, Senekal M. Application and interpretation of multiple statistical tests to evaluate validity of dietary intake assessment methods. Nutr J. 2015 Apr 22;14:40. 
58. Tabacchi G, Filippi AR, Amodio E, Jemni M, Bianco A, Firenze A, et al. A metaanalysis of the validity of FFQ targeted to adolescents. Public Health Nutr. 2016 May;19(7):1168-83.

59. Ambrosini GL, de Klerk NH, Musk AW, Mackerras D. Agreement between a brief food frequency questionnaire and diet records using two statistical methods. Public Health Nutr. 2001 Apr;4(2):255-64.

60. Kirkpatrick SI, Baranowski T, Subar AF, Tooze JA, Frongillo EA. Best Practices for Conducting and Interpreting Studies to Validate Self-Report Dietary Assessment Methods. J Acad Nutr Diet. 2019 Nov;119(11):1801-16.

61. Svensson A, Waling M, Backlund C, Larsson C. Overweight and Obese Children's Ability to Report Energy Intake Using Digital Camera Food Records during a 2-Year Study. Journal of nutrition and metabolism. 2012;2012:247389.

62. Basiotis PP, Welsh SO, Cronin FJ, Kelsay JL, Mertz W. Number of days of food intake records required to estimate individual and group nutrient intakes with defined confidence. J Nutr. 1987 Sep;117(9):1638-41.

63. Lancaster R, Radd-Vagenas S, Fiatarone Singh M, Noble Y, Daniel K, Mavros Y, et al. Electronic food records among middle-aged and older people: A comparison of self-reported and dietitian-assisted information. Nutrition \& Dietetics. 2020.

64. Cade J, Thompson R, Burley V, Warm D. Development, validation and utilisation of food-frequency questionnaires - a review. Public Health Nutr. 2002 Aug;5(4):567-87.

65. Ngo J, Gurinovic M, Frost-Andersen L, Serra-Majem L. How dietary intake methodology is adapted for use in European immigrant population groups - a review. Br J Nutr. 2009 Jul;101 Suppl 2:S86-94. 

Chapter 6

General discussion 


\section{Summary of the results from previous chapters}

This thesis investigated approaches to improve the efficiency and accuracy in collecting and handling large-scale dietary data, specifically, for the Dutch National Food Consumption Surveys (DNFCS). Firstly, we investigated possible simplifications of the method that is currently applied in the survey by removing unnecessary steps, such as less important food details, and a more simple recipes function. Secondly, new possibilities for incorporating selfadministered tools (i.e. smartphone apps) in the future surveys were explored. The collected chapters provided multidimensional evidence for constructing a self-administered smartphone app for dietary data collections in DNFCS. The learned lessons can also be useful for other large-scale dietary studies that are interested in collecting information on all foods and beverages consumed.

Simplifications on methodologies used in current DNFCS were evaluated in chapter 2 and 3. In chapter 2, the impact of less detailed characterization of consumed foods was simulated. One third of the total food descriptors used in the data collection were identified as less important in determining the nutrient intake distributions of the population. The deletion of those descriptors could potentially contribute to around 1000 hours reduction in the collection and handling of dietary data. The majority $(80 \%)$ of the differences between percentile estimates of the population nutrient intake distributions ranged from $0 \%$ to $1 \%$ before and after facet deletion. On the other hand, chapter 3 addressed the methodological simplification of collecting information on mixed dish consumption. The direct use of standard recipes without asking for details on deviations from the standard could greatly reduce the complexity of the recipe pathway, and avoid the appearance of detailed questions of which the participants that do not cook themselves often do not know the answer. A minor impact on the nutrient intake and food group consumption distributions was observed primarily due to the relatively low mixed meal consumption in the Netherlands.

Chapter 4 and 5 focused on evaluating technological developments and validations on recent smartphone food record applications. In chapter 4, the content analysis of recipe functions in popular commercial apps showed a varied functional design and displayed differences in nutrient contents of selected recipes among the apps, which were mostly due to the underlying food composition databases (FCDs). Moreover, the lack of application of yield and retention factors affected the intake estimation of heat-sensitive nutrients in certain dishes. A systematic review and meta-analysis of validation studies conducted on smartphone apps in chapter 5 revealed that energy intake derived from self-administered by apps in real-life settings were underestimated compared to more-established reference methods in general. Differences in energy intake were smaller in validation studies in which the same FCD was used in the app and reference method. 


\section{Reflections on the study findings}

\section{Importance of food descriptions}

Chapter $2 \& 3$ have illustrated that a reduction of food descriptors and the use of standard recipes without modifications could reduce the length of the interview and ease the data handling, without much impact on population nutrient intake distributions. However, apart from estimating food consumption and nutrient intakes, the collection of dietary data with adequate details makes other use of the data possible, such as assessing exposures to harmful substances (e.g. heavy metals, mycotoxins and acrylamide) (1). Information on the level of chemical exposures provides evidence for risk assessment and management of a safe and healthy food environment (2). Therefore, the usability of NFCS in estimating food safety exposure should also be considered in pursuit of a simpler methodology. Another use of food descriptors is to guide the food selection process completed by the participant themselves in self-administered methods. Findings from previous usability studies suggested that the lack of food descriptors poses difficulties in finding a specific food item within a database, resulting in a higher chance of selecting generic food items (3-5). Moreover, in chapter 5 it was speculated that this lack of guidance in apps is one of reasons for the general underestimation of the food consumption. In summary, the existence of a certain level of food details in self-administered methods enables the acquisition of useful information and provides better guidance for food selection.

Compared to web-based or computer-based dietary assessment methods, food records based on smartphones could benefit from technologies like barcodes or image capturing. For commercial foods or recipes, the detailed questions on brand names, packaging materials or other related information could be automatically captured with a simple scan, which could reduce the time and effort needed for text input, and prevent making mistakes in choosing the foods from a list (6). Note that the successful operation of this automatic linkage is built under the premise of an established pathway between the food items and food product databases (preferably with updated country-specific food products) that stores the barcodes and associated product information (7).

\section{Strategies in recording mixed dish intake}

Calculating nutrient contents of ingredients in cooked meals without yield and retention factors could lead to a large extent of misestimation in the intake of heat-sensitive nutrients (chapter 4). Due to the complexity of incorporating conversion factors into individual ingredients, chapter 3 investigated if standard recipes could be used directly without modifications. By already taking yield and retention factors into account, the source of error would mainly come from the differences in ingredient composition with the mixed dishes actually consumed. The extent of nutrient misquantification and food group misclassification 
at the population group level depends on the proportion of food consumed through mixed dishes, which was found to be rather low (10\%) in the Netherlands, causing an unnoticeable impact to the nutrient distribution at the population level. However, there was a larger difference found in certain food group intake distributions when using the standard recipes, indicating their limited suitability in reflecting the ingredients and quantities people used in real-life situations. Similar to findings from Tucker et al., mixed dishes prepared in real-life settings were much simpler than the recipes from the Internet or cookbooks $(8,9)$. Hence, the representation of standard recipes could be improved taking a large range of both cookbooks and real-life recipes into account, and incorporate functions that allow a certain level of customisation to the standard recipes (e.g. potential ingredients with checkboxes). The results from chapters $3 \& 4$ fill a gap in the limited evidence on the impact of errors when reporting mixed dish intake (10), and hopefully raises the awareness of app developers to take these factors into consideration in future app development.

\section{Importance of Food Composition Databases (FCDs)}

Commercial food products on the market are evolving rapidly, including the introduction of new products and modifications of existing products $(11,12)$. The reflections of these factors might differ across different FCDs. FCDs have shown to be one of the most influential determinants in comparing energy and nutrient intakes among different commercial apps (chapter 4), and in explaining discrepancies in apps and their reference methods across validation studies (chapter 5). Energy and macronutrients were underestimated by apps when three recipes were entered in apps and compared to calculations based on the Dutch National FCD (NEVO) by researchers (chapter 4). Griffith et al. also found underestimation of energy and nutrient using apps when compared the US Department of Agriculture's (USDA) National Nutrient Database (13). In contrast, Ferrara et al. found most of the apps tended to overestimate intake greatly (14), while Chen et al. found a balanced over- and underestimation among apps compared to USDA's FCD (15). The meta-analysis of validation studies in chapter 5 revealed that although underreporting (intentionally/unintentionally) by participants was the main contributor for the underestimation of all apps, studies that used different FCDs for the app and the reference method had higher discrepancies in intake estimations than those with the same FCDs.

The explanations for the discrepancies between commercial and national FCDs were that commercial apps might use FCDs from the country where they were developed, they might be more frequently updated in terms of commercial food products than national FCDs, but also have a higher chance of false information from crowdsourcing (15). Furthermore, the availability of micronutrient information in general was limited in apps, while most national FCDs have a rather complete nutrient profile. Proactive approaches to supplement commercial FCDs with nutrient information from national FCDs have been undergoing (16). 
Careful considerations should be made when harmonising different FCDs, since they might differ in various aspects. For example, the difference in nutrient expressions are highly likely to exist, which requires inspections and adjustment before integrating (17).

\section{Reflections on methodology:}

\section{Machine learning and the utility of large datasets}

Machine learning has been gradually incorporated into the dietary assessment area in recent years, with the main application in automatic image and spoken language recognition. Alternatively, machine learning could also be a suitable technique to predict important features (e.g. identify and reduce detailed questions) and automate certain tasks (e.g. link food to FCDs) that were mostly done manually, making use of existing data for training the algorithms (chapter 2). Another potential use could be to reduce the number of items available in the food/recipe list according to their popularity from previous surveys. Participants' inclination of answers or food choices could be differentiated based on their socioeconomic status or other personal characteristics. This information would be useful for developing customised survey protocols targeted to different population groups.

A limitation of machine learning is that the results are only applicable to the same instrument components and design. Specifically, the reduction of facets in chapter 2 was only limited to the FCD tested, in this case, the NEVO 2011/3.0. With an updated or different FCD, the proposed facet reduction might lead to different results. Besides, with the addition of new technologies and functions, the convenience of getting specific detailed information also differs. Hence, it is needed to apply a similar study protocol as in chapter 2 with each new addition of technologies and functions.

Exploiting an existing large-scale dataset for potential methodological improvement, as in chapter $2 \& 3$, has not often been found in researches developing new dietary assessment methods. Especially national surveys that have the advantage of already collected data from previous survey rounds, could consider manipulating the data somehow in understanding the utilisation of certain features or options. Cautions for manipulating large dataset should be made. Firstly, due to the complex nature of dietary intake and the detailed information collected from dietary surveys, factors that might influence the nutrient outcome should all be carefully considered when simulating procedures that were aimed to be applied automatically in real-life (e.g. apply conversion factors for calculating cooked amount). Secondly, error checking for large datasets can be problematic. Preparing a randomly selected sample dataset can be more efficient in testing the protocol. Thirdly, the limitations and assumptions in the data are reproduced, and it is well-known that reported dietary data collected from the survey always includes error. 


\section{Best practices for reporting and evaluating new dietary assessment tools}

Although a fast-growing industry of technology-assisted dietary assessment provides a wide range of selections for specific study purposes, the accuracy of the new tools is often unknown due to a lack of proper validation studies. A method that has high validity is capable of providing a useful measurement for a given purpose and has an established internal and external validity (18-20). Hence, the validity and reliability of these tools needs to be further explored with a proper evaluation strategy (21). A detailed guideline for reporting validation studies (STROBE-nut, Strengthening the Reporting of Observational Studies in Epidemiology Statement-nutritional epidemiology extension) may improve reporting of epidemiological and validation studies involving dietary assessment methods and enhance the quality of the published evidence (21). A checklist adapted from STROBE-nut with more specific guidance on the study design and results interpretation was proposed by Kirkpatrick et al (19). Another guideline developed by Eldridge et al. based on STROBE-nut consists of aspects that are more specific for reporting and validating technology-based tools (22).

As we found in chapter 5, none of the validation studies of dietary assessment apps used recovery biomarkers as their reference measure. Although they were identified as the optimal approach for measuring true intake, their limitations in cost and available nutrients have led to a reliance on the measurement of relative validity for most studies (23). The complex and dynamic nature of dietary intake contributes to difficulties in evaluating relative validity. For example, both the test and reference method might all be subjected to self-reporting errors, and the effect of using both methods might differ with using the test method only. So careful considerations on the allocated period for each method and overlaps of periods of different methods are needed to avoid learning effects in the test and reference methods. Besides, as we found in chapter 5, it can be difficult for some studies to unify the FCD used in the test and reference method. As described before in chapter 5, a larger difference between the methods using different FCDs than methods with unified FCDs has been found.

Apart from nutrients, insight in the validity of food group recordings is needed if assessing food group consumption is the purpose of the tool. This can for example be the case if consumption needs to be compared to food-based dietary guidelines, which have become more useful compared to nutrient-based guidelines in disseminating healthy eating to the public (24-29). Moreover, insight in the validity of consumption of food groups is also useful to trace back to the underlying cause of limited validity for energy and nutrients. Therefore, it is advised that the future reporting of validation studies should incorporate food group comparisons between the test and the reference method. Moreover, the investigation of omissions and intrusions of specific food items in tools can be an alternative method of comparison and could provide even more detailed insights for the source of measurement 
error. Also, the discrepancy of portion size estimation by the test and reference methods is another main source of error worth comparing.

Different levels of validity might exist for one tool in different population groups (10). Typically, a wide range of population groups is included in the sample population for NFCS (30). Hence, validation studies in a diverse population for NFCS are needed to establish external validity (31). Specifically, the practicality of technology-assisted method might be limited in segments of the population who have low e-literacy levels or motivation, leading to a weakened capacity to identify food items or portion sizes and a larger drop-out $(32,33)$.

From the meta-analyses in chapter 5 , it became clear that observed variation in differences in intake between methods has been rarely discussed for practical relevance. For instance, the Bland-Altman analysis has been applied more frequently in evaluating new methods in recent years due to its ability in detecting the presence and direction of bias at the group level, and the extent of its variation at the individual level (34). However, most studies focussed on interpreting the average bias at the group level, while the practical relevance of the individual variance (the width of limits of agreement) has rarely been assessed. In the included studies the width of the limits of agreement ranged from $447 \mathrm{kcal}$ to $3263 \mathrm{kcal}$ across studies. It is therefore advised to define acceptable limits of the variation in both the group and individual level a priori taking the desired use of the dietary assessment method into account.

Before conducting a validation study of a new dietary assessment tool, other types of evaluation are very useful during the development process. For example, the usability study on ASA24 ( Automated Self-Administered Dietary Assessment Tool) found that certain usability issues might limit the participation rate in a group of low-income participants. Participant experiences with certain features could be collected from usability studies, such as probes that could exacerbate or reduce social desirability biases $(19,35)$. A tool designed with feedbacks from users will eventually lead to better cooperation, which will, in turn, translate to a better quality of the data. Therefore, by taking usability issues into account, customised dietary assessment methods based on respondent characteristics (e.g. educational status, physiological status, geographical location, technology use) can be developed and would potentially improve the validity of the test method.

\section{Aspects (not from chapters) that are important to consider when moving from interviewer-administered $24 \mathrm{~h}$ dietary recalls to self-administered smartphone food records:}

\section{Trend of smartphone usage/data privacy}

ICT-based technologies (computers, smartphones) are more expensive platforms than pen and paper methods for dietary assessment from the user perspectives, and were deemed 
inaccessible for groups of lower socioeconomic status one decade ago (36). However, the increased coverage and ubiquity of worldwide smartphone ownership in the past ten years indicated that the affordability is of less concern and more digital devices like smartwatches and tablets are also penetrating in our daily life (18). This trend has fostered the increased access to innovative methods for assessing dietary intake. Specifically, 98 percent of Dutch households had internet access in 2018, putting the Netherlands at the forefront within Europe. The Netherlands also ranks among the European top in terms of high-speed broadband connectivity, mobile internet usage and maturity of the Mobile Health market (37). This wide application of the internet and mobile devices provides a relatively convenient start-up for implementing surveys using smartphones. Still, the level of technology-literacy of particular population groups needs to be taken in careful consideration.

Besides, the capacity of apps and other devices in monitoring other health behaviours and indicators (e.g. physical activity, sleep, heart rate, etc.) poses new opportunities for collecting a complete personal lifestyle and health profile (38). The large-scale data serves as a complement to traditional surveillance studies that could reveal new insights about the interrelationships between the environment, society and health behaviours. Data sharing partnerships between research institutions and industries might be needed for certain aims of research (39). However, at the same time, this poses challenges in ethical issues and protecting user privacy. Also the threshold of access to data on the individual level differs across countries, some countries in Europe having stricter privacy laws than other countries (40). Citizen concerns for data security also differ, for example, Swiss citizens are more concerned than citizens in the Netherlands (37). Careful considerations should be made in terms of providing standards for anonymizing activity data and transparent explanations on the use of data to the participants (39).

\section{Cost implications}

The use of interviewer-assisted food consumption surveys with much detail in food description is labour intensive and costly, which led to an exploration of the development of cost-effective technologies. This requires high investment in the early stage of the app development and testing, depending on available resources in financial, logistical and staff conditions. Once the app is ready, cost and time can be saved in organizing the study, collecting and handling data, as well as calculating dietary intakes, potentially leading to a return on investment (41). The decreased cost of data collection could enable the inclusion of more people into large-scale studies, making the study sample to be more representative of the general population.

Still, despite that removing interviewers might reduce errors related to contact bias, it may introduce additional challenges and different sources of error, causing a declined quality of the collected data (42). As the complexity of interaction with technology increase, it is 
reasonable to expect additional cost for technical support and training of participants, which have shown to improve user cooperation and proficiency (43). As seen in the selfadministered 24HR ASA24, on-demand technical assistance was available to ensure the data quality and participant retention (44). In general, there is a lack of information on costs associated with the development and implementation of new technologies in a survey setting (45). The evaluation of costs with respect to each aspect for a new method in comparison with the traditional methods could provide additional input for decision-making (45).

\section{International harmonization}

In order to develop collaborative strategies to optimize the health of the European populations, the collection of comparative food consumption data across Europe by a common framework of procedures and tools has been suggested by EFCOSUM (European Food Consumption Survey Methods) project and later validated in the 'European Food Consumption and Validation' (EFCOVAL) project. In addition, European Food Safety Authority (EFSA) emphasised the importance of pan-European dietary exposure assessments from harmonized the food consumption surveys (46). However, the differences in culture, reluctance to change currently used methods, organization structure and budgets for survey conduction are the limiting factors for methodological harmonization across countries. It was suggested that complete standardization should not be strived for at the cost of overall data quality in any individual country (40). Hence, a compromise between the level of harmonization and the practical context within each country should be reached. Although GloboDiet has been suggested as the 'first choice' instrument for data collection, the potential cost of its adaptation has prevented the use of it in some countries. Besides, other methodological aspects have also contributed to incomparability across countries, such as differences in FCD and its included nutrients, age group categories, etc. (47). Hence, the exploration of a more cost-effective method might provide new opportunities for a better future harmonization across countries. Due to the lack of validations and applications to particular population groups, smartphones have not yet been used for dietary data collection in any of the NFCS in European countries (47). The early initiative of collecting dietary data using a smartphone app in the DNFCS, taking advantage of the ever-growing smartphone penetration in the Netherlands, could provide insights for other countries that are aiming at the same direction and have expected increased use of smartphones.

\section{Future directions}

The self-reporting bias in traditional dietary assessment methods is the most worrying source of bias. Despite its limitations, self-reported intake could provide necessary detailed information about the complexity of what individuals consume. Such information is critical for providing information about dietary patterns and diet quality in order to evaluate questions such as whether intakes are consistent with recommendations or associated with health 
outcomes (27). Technology involvement can only solve certain level of unintentional underreporting $(41,48)$, while intentional underreporting cannot be easily solved. As long as the participants are aware of being monitored, the tendency to alter their diet is inevitable, especially for prospective methods (3). Until now, none of the self-administered methods has shown significant improvements in accuracy, with most of them underestimating dietary intake compared to traditional methods (30). With this in mind, parallel efforts should be put into searching for more convenient technologies and advancing statistical models that could adjust for measurement error, using data from validation studies with objective measures (e.g. recovery biomarkers).

A participation rate of less than $50 \%$ was found in the majority of the countries that have conducted NFCS $(40,49)$, with most countries relying on interviewer-assisted dietary assessment methods for current survey collections. The future participation rate was predicted to drop further if the survey methodology could not keep up with the speed of technological development (50). In general, increased compliance and willingness in using technologyassisted methods has been found in previous usability studies, due to more efficient data input, process, and flexibility in registering intake at their own convenience (51). However, there were varying levels of receptivity in a wide population group using self-administered methods targeted to large-scale data collection (e.g. ASA24)(44, 52, 53). In addition, compared to people who voluntarily use apps for dietary self-monitoring, people who were invited by a third party might not understand the purposes of the study and the importance of correct and precise recording, or have limited knowledge about their food consumption. Continued investigation on incorporating new technologies into large-scale dietary monitoring systems is an essential step for developing sufficiently accurate, cost-saving yet easy to participate future surveys.

A planned methodological change in NFCS would inevitably constitute a change in data collected, affecting the continuity of results from different survey waves, which would impair the estimation of the population intake trends over time (54). Bridging studies that investigate both methods in parallel for a sample of the population could potentially reveal the systematic bias between the methods. Compatible and comparable results from new and old method would also enable the implementation of a multi-modal approach for new survey collections, which would offer the respondents the option of either an interviewer-administered or a selfadministered survey (30).

The use of food records is usually associated with behavioural change, which was considered as a disadvantage for nutrition monitoring in many countries. However, with the incorporation of barcode scanning, and potential use of image recognition and analysis, the practicality of using an app for more days of food recording might be possible, and users might be less likely to alter their diet for prolonged periods. However, inconsistent evidence 
for the long-term use has been found, either more underreporting due to boredom or fatigue (55), or increased familiarity and a better performance in using the app (56). Features that could eliminate repetitive actions ( e.g. saving favourite or previous food items) could potentially reduce the extent of underreporting (57). More research on how food recording differs on the progression of the app use is warranted.

The use of currently available technology may not necessarily reduce all respondent burden. Although barcode scanning could automate the data entering to some extent, they are only applicable to branded food products for which the packages are available to the respondent. Text input remains the main method, which might impact the level of convenience in using the apps (58). The current incorporation of image-taking in technology-based tools makes it possible to omit food identification and amount estimation from respondents (22). These functions were proven to be useful in facilitating memory, avoiding underreporting, and ease the recording process, and might benefit most the population groups with low technology literacy (59). Especially automatic image recognition provides obvious advantages by reducing both respondent and researcher burden (60), and might be the future mainstream with the advancement in computer vision and deep learning. However, current development in image recognizing is not mature enough to be fully automated. Enormous amounts of pictures of foods and dishes are required as the input for algorithm training. The intra- and inter-individual variability with which food is prepared, served, and consumed in free-living situations brings up the levels of complexity for accurate recognition (18).

Current image-based apps still require the supplementation of descriptive information and huge data-handling efforts from researchers, meaning that participant burden may not be sufficiently reduced to offset the additional costs of extra time of researchers in the current situation (61). The evaluations of these methods were mostly conducted in controlled settings, the feasibility outside of controlled settings needs further evaluation. Hence, the full transfer from text to images would only be feasible when fully automated image recognition and analysis is achievable (62), and their requirements on the digital environment are within the technological capacity of the average consumer device (10).

Using smartphone apps for dietary intake measurement is a promising future for nutrition monitoring, given the increased penetration of smartphone use worldwide and its capability to incorporate technological features. In Figure 1, a workflow is given for developing and testing a new dietary assessment method for the use of a NFCS based on the current process of app development in the DNFCS. In the beginning phase, factors such as estimated cost and the collection of other dietary components, such as time/place of consumption need to be considered. Furthermore, taking advantages of existing results of previous surveys, as presented in chapter 2 and 3 of this thesis, provides useful evidence for methodological development. Experiences from other studies serve as essential references when integrating 
new technologies into nutrition surveys. An iterative process of developing and testing will ensure an user-adapted tool to be produced. During the developing phase, the affiliate components such as FCDs should be kept representative of the country-specific diet and equipped with information on both generic and branded food items. An effective participant training program and data cleaning protocol should be prepared. The usability and validity of the tool assessed in different population groups, together with bridging studies between the old and the new method are necessary to ensure the consistency of results before and after the methodological change.

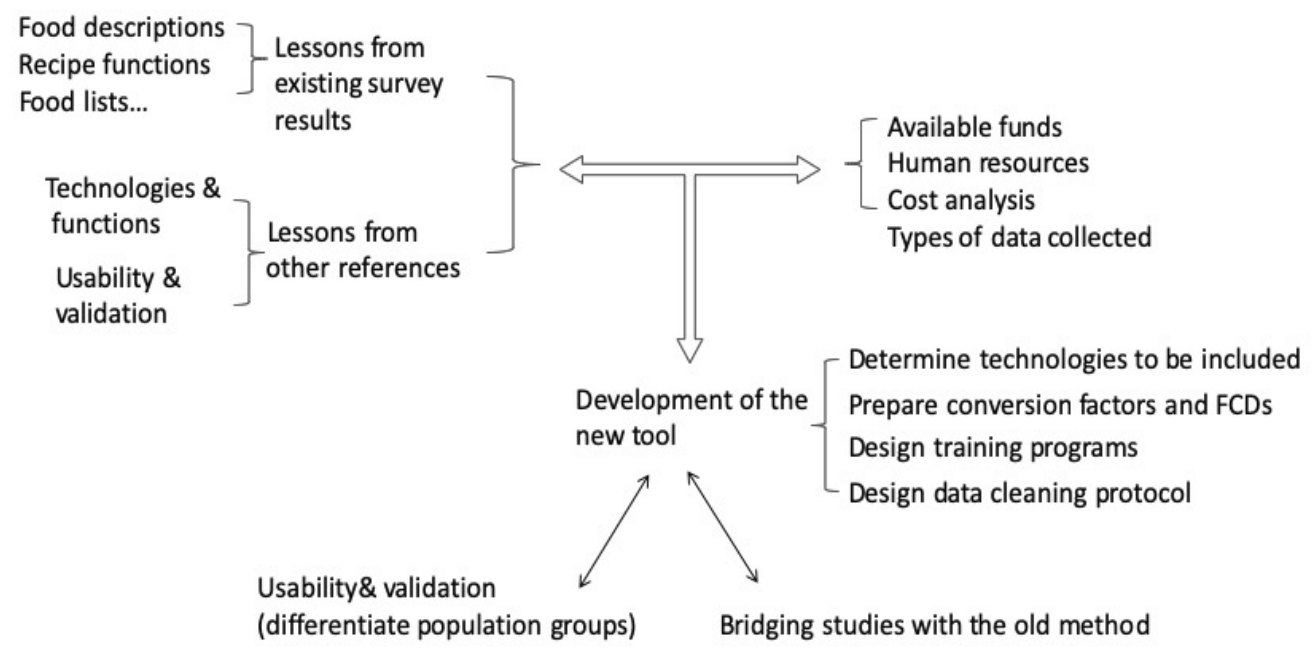

Figure 1. Process of dietary assessment tool development for NFCS 


\section{Conclusions}

Although being acknowledged as error-prone, self-reported dietary intake at the national level has reaffirmed its value as an essential scientific foundation for developing public health policies, food-based guidelines, and understanding diet and health relationships $(54,63)$. In order to improve the accuracy and reduce the burden of obtaining dietary data, the dietary assessment field is working on enhancing existing methods, developing innovative instruments using new technologies, and incorporating statistical methods for error adjustments (64-67). This thesis concluded that a reduced amount of food descriptors and a simplified recipe pathway in $24 \mathrm{HR}$ does not have a large impact on the population nutrient intake distributions and could potentially reduce the cost of future interviewer-administered 24HRs. The findings thus indicated that the collection of certain details could be omitted for developing smartphone apps built for self-administered food records. On the other hand, whether a self-administered food record tool has sufficient accuracy for dietary monitoring needs to be determined. No biomarker-based validity studies are available yet; and relative to other dietary assessment methods there seems to be more underreporting. Insight in the underlying causes of this underestimation and variations in accuracy is largely lacking.

Smartphone apps for dietary assessment have rarely been tested for large-scale studies, especially for NFCS. Several main reasons might explain the lack of such explorations, including varied acceptability among different population groups, the susceptibility to behavioural change using prospective methods, and insufficient insight in the accuracy of smartphone food records. Still, with the undeniable trend towards more automated procedures in dietary assessment, a self-administered method for NFCS is likely to take over the interviewer-administered method in the near future. Several strategies to cope with the challenges in developing and testing self-administered methods for large surveys exist. Firstly, data mining of previously collected food consumption data is a cost-effective approach that could potentially reveal useful information. Secondly, the sources of errors using the new method should be traced to enable further adjustments of the tool. Thirdly, the design of validation studies should comply with the established recommendations and cover all population groups of interest to the survey. 


\section{Reference}

1. Mengelers M, de Wit L, Boon P, Franz E, Bouwknegt M, de Jonge R, et al. How safe is our food? Background report to 'What's on our plate? Safe, healthy and sustainable diets in the Netherlands.'. Bilthoven National Institute for Public Health and the Environment 2016.

2. EFSA. General principles for the collection of national food consumption data in the view of a pan-European dietary survey. EFSA Journal. 2009;7(12):1435.

3. Illner AK, Freisling H, Boeing H, Huybrechts I, Crispim SP, Slimani N. Review and evaluation of innovative technologies for measuring diet in nutritional epidemiology. Int J Epidemiol. 2012 Aug;41(4):1187-203.

4. Zimmerman TP, Hull SG, McNutt S, Mittl B, Islam N, Guenther PM, et al. Challenges in converting an interviewer-administered food probe database to self-administration in the National Cancer Institute Automated Self-administered 24-Hour Recall (ASA24). Journal of food composition and analysis : an official publication of the United Nations University, International Network of Food Data Systems. 2009;22(Suppl 1):S48-S51.

5. Koch SAJ, Conrad J, Hierath L, Hancock N, Beer S, Cade JE, et al. Adaptation and Evaluation of Myfood24-Germany: A Web-Based Self-Administered 24-h Dietary Recall for the German Adult Population. Nutrients. 2020;12(1):160.

6. Steele R. An overview of the state of the art of automated capture of dietary intake information. Crit Rev Food Sci Nutr. 2015;55(13):1929-38.

7. Maringer M, Wisse-Voorwinden N, Veer PV, Geelen A. Food identification by barcode scanning in the Netherlands: a quality assessment of labelled food product databases underlying popular nutrition applications. Public Health Nutr. 2018 Jul 2:1-8.

8. Tucker KL, Bianchi LA, Maras J, Bermudez OI. Adaptation of a food frequency questionnaire to assess diets of Puerto Rican and non-Hispanic adults. American journal of epidemiology. 1998 Sep 1;148(5):507-18.

9. Ngo J, Gurinovic M, Frost-Andersen L, Serra-Majem L. How dietary intake methodology is adapted for use in European immigrant population groups - a review. $\mathrm{Br}$ J Nutr. 2009 Jul;101 Suppl 2:S86-94.

10.Prinz N, Bohn B, Kern A, Püngel D, Pollatos O, Holl RW. Feasibility and relative validity of a digital photo-based dietary assessment: results from the Nutris-Phone study. Public Health Nutrition. 2019;22(7):1160-7.

11.Mueller S, Szolnoki G. The relative influence of packaging, labelling, branding and sensory attributes on liking and purchase intent: Consumers differ in their responsiveness. Food Quality \& Preference. 2010;21(7):0-783.

12. World food consumption patterns - trends and drivers. European Commission, 2015.

13. Griffiths C, Harnack L, Pereira MA. Assessment of the accuracy of nutrient calculations of five popular nutrition tracking applications. Public Health Nutr. 2018 Jun;21(8):1495-502. 
14.Ferrara G, Kim J, Lin S, Hua J, Seto E. A Focused Review of Smartphone DietTracking Apps: Usability, Functionality, Coherence With Behavior Change Theory, and Comparative Validity of Nutrient Intake and Energy Estimates. JMIR Mhealth Uhealth. 2019 May 17;7(5):e9232.

15.Chen J, Cade JE, Allman-Farinelli M. The Most Popular Smartphone Apps for Weight Loss: A Quality Assessment. JMIR Mhealth Uhealth. 2015 Dec 16;3(4):e104.

16.Carter MC, Hancock N, Albar SA, Brown H, Greenwood DC, Hardie LJ, et al. Development of a New Branded UK Food Composition Database for an Online Dietary Assessment Tool. Nutrients. 2016 Aug 5;8(8).

17.Leclercq C, Valsta LM, Turrini A. Food composition issues--implications for the development of food-based dietary guidelines. Public Health Nutr. 2001 Apr;4(2b):67782.

18. Rollo ME, Williams RL, Burrows T, Kirkpatrick SI, Bucher T, Collins CE. What Are They Really Eating? A Review on New Approaches to Dietary Intake Assessment and Validation. Current Nutrition Reports. 2016;5(4):307-14.

19.Kirkpatrick SI, Baranowski T, Subar AF, Tooze JA, Frongillo EA. Best Practices for Conducting and Interpreting Studies to Validate Self-Report Dietary Assessment Methods. J Acad Nutr Diet. 2019 Nov;119(11):1801-16.

20.Barry AE, Chaney B, Piazza-Gardner AK, Chavarria EA. Validity and reliability reporting practices in the field of health education and behavior: a review of seven journals. Health education \& behavior : the official publication of the Society for Public Health Education. 2014 Feb;41(1):12-8.

21.Lachat C, Hawwash D, Ocke MC, Berg C, Forsum E, Hornell A, et al. Strengthening the Reporting of Observational Studies in Epidemiology - nutritional epidemiology (STROBE-nut): An extension of the STROBE statement. Nutr Bull. 2016 Sep;41(3):240-51.

22.Eldridge AL, Piernas C, Illner AK, Gibney MJ, Gurinovic MA, de Vries JHM, et al. Evaluation of New Technology-Based Tools for Dietary Intake Assessment-An ILSI Europe Dietary Intake and Exposure Task Force Evaluation. Nutrients. 2019 Jan;11(1). 23.Jenab M, Slimani N, Bictash M, Ferrari P, Bingham SA. Biomarkers in nutritional epidemiology: applications, needs and new horizons. Hum Genet. 2009 Jun;125(56):507-25.

24.Julian-Almarcegui C, Bel-Serrat S, Kersting M, Vicente-Rodriguez G, Nicolas G, Vyncke K, et al. Comparison of different approaches to calculate nutrient intakes based upon 24-h recall data derived from a multicenter study in European adolescents. Eur J Nutr. 2016 Mar;55(2):537-45.

25.Millen BE, Abrams S, Adams-Campbell L, Anderson CA, Brenna JT, Campbell WW, et al. The 2015 Dietary Guidelines Advisory Committee Scientific Report: Development and Major Conclusions. Adv Nutr. 2016 May;7(3):438-44. 
26. Monteiro CA, Cannon G, Moubarac JC, Martins AP, Martins CA, Garzillo J, et al. Dietary guidelines to nourish humanity and the planet in the twenty-first century. A blueprint from Brazil. Public Health Nutr. 2015 Sep;18(13):2311-22.

27.Subar AF, Freedman LS, Tooze JA, Kirkpatrick SI, Boushey C, Neuhouser ML, et al. Addressing Current Criticism Regarding the Value of Self-Report Dietary Data. The Journal of nutrition. 2015 Dec;145(12):2639-45.

28.Micha R, Khatibzadeh S, Shi P, Andrews KG, Engell RE, Mozaffarian D, et al. Global, regional and national consumption of major food groups in 1990 and 2010: a systematic analysis including 266 country-specific nutrition surveys worldwide. BMJ Open. 2015 Sep 24;5(9):e008705.

29.Castro-Quezada I, Ruano-Rodriguez C, Ribas-Barba L, Serra-Majem L. Misreporting in nutritional surveys: methodological implications. Nutricion hospitalaria. $2015 \mathrm{Feb}$ 26;31 Suppl 3:119-27.

30.Amoutzopoulos B, Steer T, Roberts C, Cade J, Boushey C, Collins C, et al. Traditional methods $\mathrm{v}$. new technologies-dilemmas for dietary assessment in large-scale nutrition surveys and studies: a report following an international panel discussion at the 9 th International Conference on Diet and Activity Methods (ICDAM9), Brisbane, 3 September 2015. Journal of nutritional science. 2018;7.

31.Thompson FE, Subar AF, Boushey CJ, Ferruzzi MG, Delahanty LM. Chapter 1 Dietary Assessment Methodology A2 - Coulston, Ann M. Nutrition in the Prevention and Treatment of Disease (Fourth Edition): Academic Press; 2017. p. 5-48.

32. Thompson FE, Subar AF. Dietary assessment methodology. Nutrition in the Prevention and Treatment of Disease: Elsevier; 2017. p. 5-48.

33.Tay W, Kaur B, Quek R, Lim J, Henry CJ. Current Developments in Digital Quantitative Volume Estimation for the Optimisation of Dietary Assessment. Nutrients. 2020;12(4):1167.

34.Lombard MJ, Steyn NP, Charlton KE, Senekal M. Application and interpretation of multiple statistical tests to evaluate validity of dietary intake assessment methods. Nutr J. 2015 Apr 22;14:40.

35.Subar AF, Thompson FE, Potischman N, Forsyth BH, Buday R, Richards D, et al. Formative research of a quick list for an automated self-administered 24-hour dietary recall. J Am Diet Assoc. 2007 Jun;107(6):1002-7.

36.Long JD, Littlefield LA, Estep G, Martin H, Rogers TJ, Boswell C, et al. Evidence review of technology and dietary assessment. Worldviews on Evidence-Based Nursing. 2010;7(4):191-204.

37.De Korver F. The Digital Health market in the Netherlands and Switzerland Switzerland: Embassy of the Kingdom of the Netherlands in Berne, 2019.

38.Svensson A, Larsson C. A Mobile Phone App for Dietary Intake Assessment in Adolescents: An Evaluation Study. JMIR Mhealth Uhealth. 2015 Nov 3;3(4):e93. 
39.Hicks JL, Althoff T, Sosic R, Kuhar P, Bostjancic B, King AC, et al. Best practices for analyzing large-scale health data from wearables and smartphone apps. npj Digital Medicine. 2019;2(1):45.

40.De Henauw S, Brants HA, Becker W, Kaic-Rak A, Ruprich J, Sekula W, et al. Operationalization of food consumption surveys in Europe: recommendations from the European Food Consumption Survey Methods (EFCOSUM) Project. Eur J Clin Nutr. 2002 May;56 Suppl 2:S75-88.

41.Shim J-S, Oh K, Kim HC. Dietary Assessment Methods in Epidemiologic Studies. Epidemiology and Health. 2014;36:e2014009.

42.Lancaster R, Radd-Vagenas S, Fiatarone Singh M, Noble Y, Daniel K, Mavros Y, et al. Electronic food records among middle-aged and older people: A comparison of selfreported and dietitian-assisted information. Nutr Diet. 2020.

43.Six BL, Schap TE, Kerr DA, Boushey CJ. Evaluation of the Food and Nutrient Database for Dietary Studies for use with a mobile telephone food record. Journal of food composition and analysis : an official publication of the United Nations University, International Network of Food Data Systems. 2011 Dec 1;24(8):1160-7.

44.Kupis J, Johnson S, Hallihan G, Olstad DL. Assessing the Usability of the Automated Self-Administered Dietary Assessment Tool (ASA24) among Low-Income Adults. Nutrients. 2019;11(1):132.

45.Burley V, Timmins K, Cade J, Greenwood D, Husain F, Gill V, et al. Making the best use of new technologies in the National Diet and Nutrition Survey: a review. 2015 $03 / 31$.

46.Guidance on the EU Menu methodology1 European Food Safety Authority.

47.Rippin H, Hutchinson J, Evans C, Jewell J, Breda J, Cade J. National nutrition surveys in Europe: a review on the current status in the 53 countries of the WHO European region. Food \& Nutrition Research. 2018;62.

48.Naska A, Lagiou A, Lagiou P. Dietary assessment methods in epidemiological research: current state of the art and future prospects. F1000Research. 2017;6:926.

49. Walton J. Dietary Assessment Methodology for Nutritional Assessment: A Practical Approach. Top Clin Nutr. 2015;30(1):33-46 10.1097/TIN.0000000000000018.

50.Keeble C, Baxter PD, Barber S, Law G. Participation Rates In Epidemiology Studies And Surveys: A Review 2007-2015. The Internet Journal of Epidemiology. 2016 $01 / 27 ; 14$.

51.Kroeze W, Werkman A, Brug J. A systematic review of randomized trials on the effectiveness of computer-tailored education on physical activity and dietary behaviors. Ann Behav Med. 2006 Jun;31(3):205-23.

52.Thompson FE, Dixit-Joshi S, Potischman N, Dodd KW, Kirkpatrick SI, Kushi LH, et al. Comparison of Interviewer-Administered and Automated Self-Administered 24-Hour Dietary Recalls in 3 Diverse Integrated Health Systems. American journal of epidemiology. 2015 Jun 15;181(12):970-8. 
53.Kirkpatrick SI, Gilsing AM, Hobin E, Solbak NM, Wallace A, Haines J, et al. Lessons from Studies to Evaluate an Online 24-Hour Recall for Use with Children and Adults in Canada. Nutrients. 2017 Jan 31;9(2).

54.Archer E, Hand GA, Blair SN. Validity of U.S. nutritional surveillance:National Health and Nutrition Examination Survey caloric energy intake data, 1971-2010. PLoS One. 2013;8(10):e76632.

55.Carter MC, Burley VJ, Nykjaer C, Cade JE. 'My Meal Mate' (MMM): validation of the diet measures captured on a smartphone application to facilitate weight loss. Br J Nutr. 2013 Feb 14;109(3):539-46.

56.Chen J, Berkman W, Bardouh M, Ng CYK, Allman-Farinelli M. The use of a food logging app in the naturalistic setting fails to provide accurate measurements of nutrients and poses usability challenges. Nutrition. 2019;57:208-16.

57.Weber F, Haering C, Thomaschke R. Improving the human-computer dialogue with increased temporal predictability. Hum Factors. 2013 Oct;55(5):881-92.

58.Ming Z-Y, Chen J, Cao Y, Forde C, Ngo C-W, Chua TS, editors. Food Photo Recognition for Dietary Tracking: System and Experiment. International Conference on Multimedia Modeling; 2018: Springer.

59.Gemming L, Doherty A, Kelly P, Utter J, Ni Mhurchu C. Feasibility of a SenseCamassisted 24-h recall to reduce under-reporting of energy intake. Eur J Clin Nutr. 2013 Oct;67(10):1095-9.

60.Rangan AM, O'Connor S, Giannelli V, Yap MLH, Ming L, Roy R, et al. Electronic Dietary Intake Assessment (e-DIA): Comparison of a Mobile Phone Digital Entry App for Dietary Data Collection With 24-Hour Dietary Recalls. JMIR MHEALTH AND UHEALTH. 2015 2015--2015;3(4):3-14.

61.Sharp DB, Allman-Farinelli M. Feasibility and validity of mobile phones to assess dietary intake. Nutrition. 2014 Nov-Dec;30(11-12):1257-66.

62.Martin CK, Nicklas T, Gunturk B, Correa JB, Allen HR, Champagne C. Measuring food intake with digital photography. J Hum Nutr Diet. 2014 Jan;27 Suppl 1:72-81.

63.Black AE, Goldberg GR, Jebb SA, Livingstone MB, Cole TJ, Prentice AM. Critical evaluation of energy intake data using fundamental principles of energy physiology: 2. Evaluating the results of published surveys. Eur J Clin Nutr. 1991 Dec;45(12):583-99.

64.Neuhouser ML, Tinker L, Shaw PA, Schoeller D, Bingham SA, Horn LV, et al. Use of recovery biomarkers to calibrate nutrient consumption self-reports in the Women's Health Initiative. American journal of epidemiology. 2008;167(10):1247-59.

65.Thompson FE, Subar AF, Loria CM, Reedy JL, Baranowski T. Need for technological innovation in dietary assessment. J Am Diet Assoc. 2010 Jan;110(1):48-51.

66.Freedman LS, Schatzkin A, Midthune D, Kipnis V. Dealing with dietary measurement error in nutritional cohort studies. J Natl Cancer Inst. 2011 Jul 20;103(14):1086-92.

67.Prentice RL, Huang Y. Measurement error modeling and nutritional epidemiology association analyses. Can J Stat. 2011 Sep 1;39(3):498-509. 
Acknowledgements 
Here, after 4 years and 1 month in Wageningen, my $\mathrm{PhD}$ journey has finally ended in Nov. 2020, with the pandemic of Covid-19 everywhere around the world. Although it is difficult to meet with people around this time, I still would like to mention and acknowledge those who inspired, guided, and accompanied me throughout this entire journey.

First of all, I would like to give special thanks to my supervisors, Hendriek Boshuizen and Marga Ocke. Marga has been a close contact to me throughout my entire $\mathrm{PhD}$, we had countless meetings regularly, and tackled big or small challenges together. I am deeply grateful to your patience, guidance, and trust in me, which helped me gradually gained confidence and independence in doing research. Your serious attitude towards science will always be a good example for me. Hendriek, as my promoter, provided substantial support to me in both work and life. I truly appreciate and enjoy spending time with you during tutoring hours on statistics whenever I had difficulties. I am grateful for your genuine advice on future careers. Without guidance from both of you, it would take much longer for me to finalize my study. Your understanding and caring for me during the Covid-19 lockdown has greatly comforted me and facilitated me to overcome this difficult time. Despite of your busy schedule, I appreciate that you were actively involved in each step of my study. I feel very privileged to have been reached out by my supervisors and Anouk Geelen to have the opportunity to work in both Wageningen and RIVM. Anouk, although supervised me for only my early period, I gained so much from you, and started to reflect myself occasionally, you also cared a lot with my life outside work and showed great caring to make my life easier, I am so thankful for that!

Special thanks to my reading committee: Prof Pieter van't Veer, Dr Annette Stafleu, Prof Janet Cade, and Dr Simone Eussen, for taking time to review my thesis and to attend my defence. I appreciate that you will be there for my last step of my $\mathrm{PhD}$ journey.

During this four years, I received a lot of support from people at the Division of Human Nutrition in Wageningen, I would start from Adrienne Cavelaars, thank you for your help with my financial situations, which have greatly relieved my stress during my research. Also, I would like to thank the chair of my group, Ellen Kampman, who showed a lot of encouragement and trust in me. I am always influenced by your active attitude when you are around. To Kees de Graaf, you are not my direct supervisor, but we had a great time together during the Cabaret experience, best wishes for you! Edith Feskens, even we did not win the dinner game, our memories will always be remembered by me. Alida Melse-Boonstra and Lucy Elburg, thank you very much for thinking about me when you were organizing international activities, which makes me blend in more easily and feel greatly welcomed. Moreover, my appreciations to Jasmijn Mater and Gea Brussen, you are always there for me to solve my problems. Thank you a lot! 
To my dearest colleagues in RIVM, Robert Jan de Klein, Zohreh Ghameshlou, Ido Toxopeus, Caroline van Rossum, Susanne Westenbrink, Maryse Niekerk and Arnold Dekkers even though we did not meet regularly, you are always so friendly and welcome. The game night we had in Marga's place was so much fun! Zohreh, and Maryse, thank you very much for your support of my project, and patient in answering my questions! Also, I would like to thank colleagues in the statistic group, especially José Ferreira, your kind support made it possible for me to grasp complicated statistical concepts, it was always a pleasure to chat with you! And Jan van de Kassteele, who is always there for my technical problems, thank you!

To my two dearest Masters students, Eline Nawijn and Andreja Misir. Eline you are such an easy-going person, and did not bother doing the app testing again after your graduation, I greatly appreciate that! And it was nice to see you again during the game night! Hope you have a nice time working in RIVM. Andreja, you are like a superwoman to me, managing to work and finish your thesis at the same time, you are more like a friend to me than a student. I believe you now have much more confidence in your future career in nutrition.

To my earliest friends and colleagues since I joined Wageningen and have left Wageningen already, Martin, Aregash, Masresha, Ibukun, and Apple, thank you for your warmest acceptance to me, blessings to all of you! More people later had contacts with me and we shared a lot of nice memories together, you made this department such a happy place, Maria Duenas, Tsitsi, Inga, Maria Salazar, Tesfaye, Charlotte, Katherine, Eric, Ibukun, Anita, Mahsina, Asrullah, Fusta, Jesca, Giulia, Marijke, Marion, Paulina, Adele, Lowela, Kamalita, Marielle, Pol, Iris, Anniek, Vera, Moniek, Ursula, Dessy, Duong, Aafke. My lovely officemates, Lupita, Maria Jacobo, Umi, Taymara, and Santiago, we spent the most time together, you made my stressful working hours more relaxing through casual chit chats. If I could have such nice officemates as you guys in the future, it will be a blessing for me! To my Chinese gang in HNE, Ruoxuan, Xiaolin, Danny, Max, and Cong. I am so happy to see our family is growing year by year, and feel sad of leaving at this moment. Hope you could enjoy the rest of your study and let's see each other in China someday!

To my second 'parents' in the Netherlands, Ed and Margreet, you provided a home for me for four years, welcomed me every Friday, providing physical and spiritual food. I am so blessed to know you and all of the brothers and sisters in the fellowship, Maaike, EJ, Phil, $\mathrm{Lu}$, Christina, I learned so much from all of you! Meng, Lisheng, Wenzhen, Xiaoqi, Hongli, Yangyang, Yuqing, Dongyao, Yingying, Chen, Shengxin, Weixuan, and Marlon, I was so glad to share thoughts with you, and pray with each of you.

Last but not the least, I appreciate the accompany of Ru, Tania, Sijia, Jiaqi, Danlei, Shan, Yanjun, Yu, Bingyu, Zihan, for countless dinners, trips and parties together. My time in 
Wageningen became much more colorful because of all of you! To my long-distance friends, we are connected no matter where we are, Ting, Jingyi, Jing, and Xinyuan. Finally, I wouldn't have come this far without the love and support from my parents and my relatives. My love for you will never end!

With Love,

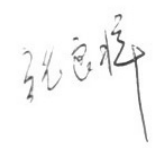


About the author 


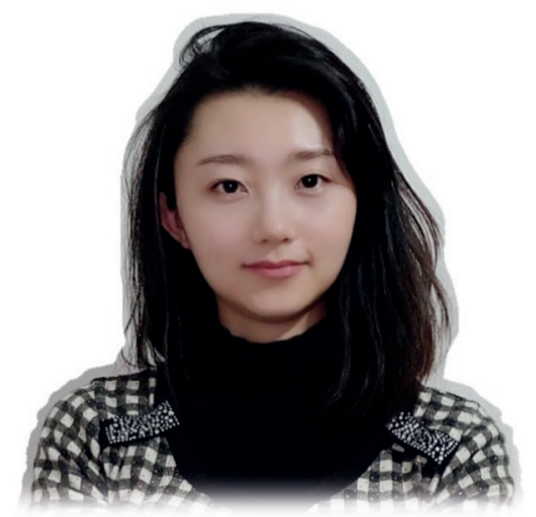

Liangzi Zhang was born on June $2^{\text {nd }}, 1991$ in Datong, China. After completing her high school in Beijing-Concord College of Sino-Canada in 2009, she started majoring in business at Western University, Canada. Two years later, she transferred to the program of nutrition and dietetics, which she was much more interested in. After completing her bachelor in 2013, she worked as a nutrition program coordinator for the university summer camp, then a food journalist in Vancouver. In 2014, She started her master's study in food science and nutrition at the University of Leeds, UK. Her master thesis was about the validation of the MyMealMate food record app, supervised by Dr. Janet Cade. After graduation, she worked as a research assistant in the Nutrition Epidemiology group for the maintenance of the New Branded UK Food Composition Database.

In October 2016, Liangzi started her Ph.D. in the Division of Human Nutrition at Wageningen University and RIVM (National Institute for Public Health and the Environment) in the Netherlands. Her project aimed to enhance the efficiency of the Dutch National Food Consumption Surveys, by eliminating unnecessary steps in the current method, and assess the possibility of incorporating new technologies into future national surveys. Besides her research activities, she supervised several nutrition epidemiology courses and theses of masters students. Liangzi presented her study by posters in WEON conference in RIVM, Max-Rubner conference in Karlsruhe. She presented orally in Dutch Nutrition Science meetings, and at the University of Cambridge and Newcastle University during the $2017 \mathrm{PhD}$ tour in the UK. Apart from her research in the Netherlands, she published three scientific newsfeeds in Chinese and completed a dietary questionnaire for a personalized nutrition program according to the Dietary Guidelines for Chinese Residents (2016). 


\section{List of Publications}

Published in peer-reviewed journals

Zhang L, Geelen A, Boshuizen HC, Ferreira J, Ocke MC. Importance of details in food descriptions in estimating population nutrient intake distributions. Nutr J. 2019;18(1):17.

Zhang L, Nawijn E, Boshuizen H, Ocke M. Evaluation of the Recipe Function in Popular Dietary Smartphone Applications, with Emphasize on Features Relevant for Nutrition Assessment in Large-Scale Studies. Nutrients. 2019;11(1).

Zhang L, Boshuizen H, Ocke M. How does a simplified recipe collection procedure in dietary assessment tools affect the food group and nutrient intake distributions of the population. Br J Nutr. 2020:1-10.

Publications in preparation

Zhang L, Misir A, Boshuizen H, Ocke M. A Systematic Review and Meta-analysis of Validation Studies Performed on Food Record Apps.

Zhang L*, Sijbrandij J*, Ocke M, Boshuizen H. Validity Measures and Statistical Modeling Choices in Biomarker-based Validation Studies: An In-depth Literature Review. *Shared first authorship. 


\section{Overview of completed training activities}

\begin{tabular}{lll} 
Discipline specific activities & Organizer and location & Year \\
\hline $\begin{array}{l}\text { Courses } \\
\text { Exposure Assessment }\end{array}$ & VLAG, Wageningen, NL & 2018 \\
$\begin{array}{l}\text { Modelling of habitual dietary intake } \\
\text { Nutritional Epidemiology Course }\end{array}$ & VLAG, Wageningen, NL & 2017 \\
Measurement Error Webinar Series & National Cancer Institute, US & 2017 \\
& & \\
$\begin{array}{l}\text { Conferences and meetings } \\
\text { WEON conference }\end{array}$ & RIVM, Bilthoven, NL \\
Dutch Nutritional Science Days & NAV, Heeze, NL \\
Menu-D meeting & Human Nutrition and Disease, & $2016-2020$ \\
Max-Rubner Conference & Wageningen, NL & 2018 \\
NUTRITION 2020 LIVE ONLINE & Max-Rubner Institute, Karlsruhe, & 2017 \\
& DE & $2018-2019$ \\
& US & 2020
\end{tabular}

General Courses and activities

VLAG PhD week

Scientific writing

Presentation workshop

$\mathrm{R}$ course

Career orientation
VLAG, Baarlo,NL

2016

WGS, Wageningen, NL

2017

Division of Human Nutrition, Wageningen, NL

RIVM, Bilthoven, NL

WGS, Wageningen, NL
2019

2019

2019

\section{Optional courses and activities}

\begin{tabular}{lll}
\hline PhD study tour UK & WUR, UK & 2017 \\
MSc course Applied Data Analysis & WUR, Wageningen, NL & 2016 \\
NAD-Paperclub & Human Nutrition and Disease, & $2016-2020$ \\
& Wageningen, NL
\end{tabular}





\section{Colophon}

The research described in this thesis was partly performed at the National institute for Public Health and the Environment (RIVM), Bilthoven.

Financial support from Wageningen University for printing this thesis is gratefully acknowledged.

Cover Design and layout: Liangzi Zhang and Mercedes Benjaminse Printed by DIGIFORCE Proefschriftmaken.nl, Wageningen, the Netherlands

Copyright $(C$ Liangzi Zhang, 2020 



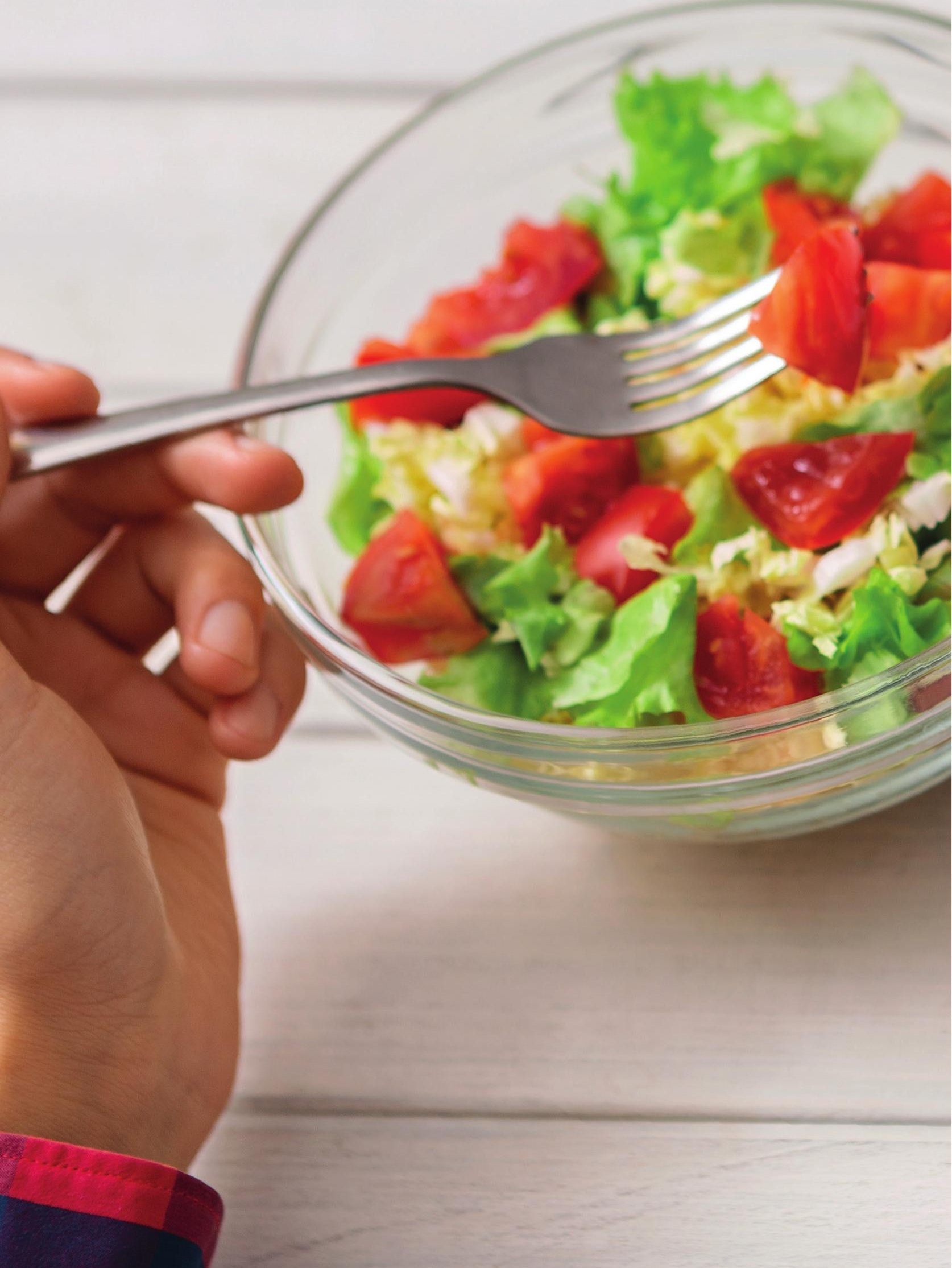

